\title{
Antenna subtraction at NNLO with hadronic initial states Initial-final configurations
}

\author{
Journal Article \\ Author(s): \\ Daleo, Alejandro; Gehrmann-De Ridder, Aude; Gehrmann, Thomas; Luisoni, Gionata \\ Publication date: \\ 2010-01 \\ Permanent link: \\ https://doi.org/10.3929/ethz-b-000017226 \\ Rights / license: \\ Creative Commons Attribution 4.0 International \\ Originally published in: \\ Journal of High Energy Physics 2010(1), https://doi.org/10.1007/JHEP01(2010)118
}




\section{Antenna subtraction at NNLO with hadronic initial states: initial-final configurations}

\section{Alejandro Daleo, ${ }^{a}$ Aude Gehrmann-De Ridder, ${ }^{a}$ Thomas Gehrmann ${ }^{b}$ and Gionata Luisoni ${ }^{b}$}

${ }^{a}$ Institute for Theoretical Physics, ETH, CH-8093 Zürich, Switzerland

${ }^{b}$ Institut für Theoretische Physik, Universität Zürich, Winterthurerstrasse 190, CH-8057 Zürich, Switzerland

E-mail: adaleo@phys.ethz.ch, gehra@phys.ethz.ch, thomas.gehrmann@physik.uzh.ch, luisonig@physik.uzh.ch

ABSTRACT: We extend the antenna subtraction method to include initial states containing one hadron at NNLO. We present results for all the necessary subtraction terms, antenna functions, for the master integrals required to integrate them over the relevant phase space and finally for the integrated antennae themselves. Where applicable, our results are cross-checked against the known NNLO coefficient functions for deep inelastic scattering processes.

KeYwords: Jets, NLO Computations, Hadronic Colliders, QCD

ARXiv EPRINT: 0912.0374 


\section{Contents}

1 Introduction 1

2 Initial-final antenna subtraction at NNLO 3

$\begin{array}{lll}3 & \text { Phase space mappings } & 10\end{array}$

4 Integration of initial-final antenna functions at NNLO 12

4.1 Tree-level $2 \rightarrow 3$ antenna functions 14

4.2 One-loop $2 \rightarrow 2$ antenna functions 16

5 Quark initiated antennae $\quad 18$

$\begin{array}{ll}5.1 \text { Quark-quark antennae } & 18\end{array}$

5.2 Quark-gluon antennae 26

$\begin{array}{lll}5.3 & \text { Gluon-gluon antennae } & 36\end{array}$

6 Gluon initiated antennae $\quad 41$

6.1 Quark-quark antennae 41

6.2 Quark-gluon antennae 45

6.3 Gluon-gluon antennae $\quad 52$

7 Rederivation of NNLO coefficient functions $\quad 60$

$\begin{array}{ll}7.1 & \text { Deep inelastic scattering: } \gamma \text { induced }\end{array}$

$\begin{array}{lll}7.2 & \text { Deep inelastic scattering: } \phi \text {-scalar induced } & 62\end{array}$

8 Conclusions and outlook $\quad 64$

A Master integrals $\quad \mathbf{6 5}$

$\begin{array}{lll}\text { A.1 Master integrals for double real radiation } & 65\end{array}$

$\begin{array}{lll}\text { A.2 Master integrals for one-loop single real radiation } & 68\end{array}$

$\begin{array}{ll}\text { B } \phi \text {-DIS coefficient functions } & 69\end{array}$

\section{Introduction}

Final states containing hadronic jets are produced at large rates at high energy particle colliders. Since the distribution of these jets relates directly to the dynamics of the hard parton-level scattering process, jet observables can be used to probe elementary particle reactions. Owing to their large production cross sections, various jet observables can be measured to a high statistical accuracy, thus making them ideal for precision studies $[1,2]$ : 
for example three-jet production in $e^{+} e^{-}$annihilation to determine the strong coupling constant $\alpha_{s}$ and for quantum chromodynamics (QCD) studies, two-plus-one-jet production in deep inelastic scattering as a probe of the gluon distribution in the proton and to measure $\alpha_{s}$, single jet inclusive production and vector-boson-plus-jet production at hadron colliders as benchmark reactions, and to measure parton distributions. Experimental data on these observables often attain an accuracy of a few per cent or better, such that meaningful precision studies must rely on theoretical predictions accurate to the same level. In perturbative QCD, this precision usually requires corrections at next-to-next-to-leading order (NNLO).

NNLO calculations of observables with $n$ jets in the final state require several ingredients: the two-loop corrected $n$-parton matrix elements, the one-loop corrected $(n+1)$ parton matrix elements, and the tree-level $(n+2)$-parton matrix elements. For most massless jet observables of phenomenological interest, the two-loop matrix elements have been computed some time ago [3-18], while the other two types of matrix elements are usually known from calculations of next-to-leading order (NLO) corrections to $(n+1)$ jet production [19-28].

The one-loop $(n+1)$-parton matrix elements contribute to $n$ jet observables at NNLO if one of the partons involved becomes unresolved (soft or collinear) [29-33]. In these cases, the infrared singular parts of the matrix elements need to be extracted and integrated over the phase space appropriate to the unresolved configuration to make the infrared pole structure explicit. Methods for the extraction of soft and collinear limits of one-loop matrix elements are worked out in detail in the literature [29-39]. Likewise, the $(n+2)$-parton matrix elements contain double real radiation singularities corresponding to two partons becoming simultaneously soft and/or collinear [40-46]. To determine the contribution to NNLO jet observables from these configurations, one has to find two-parton subtraction terms which coincide with the full matrix element and are still sufficiently simple to be integrated analytically in order to cancel their infrared pole structure with the two-loop virtual and the one-loop single-unresolved contributions. Often starting from systematic methods for subtraction at NLO [47-53], several NNLO subtraction methods have been proposed in the literature [54-64], and are worked out to a varying level of sophistication.

For observables with partons only in the final state, an NNLO subtraction formalism, antenna subtraction, has been derived in [65]. The antenna subtraction formalism constructs the subtraction terms from antenna functions. Each antenna function encapsulates all singular limits due to the emission of one or two unresolved partons between two colourconnected hard radiator partons. This construction exploits the universal factorization of matrix elements and phase space in all unresolved limits. The antenna functions are derived systematically from physical matrix elements [66-68]. This formalism has been applied in the derivation of NNLO corrections to three-jet production in electron-positron annihilation [69-72] and related event shapes [73-77], which were used subsequently in precision determinations of the strong coupling constant [78-84]. The formalism can be extended to include parton showers at higher orders [85], thereby offering a process-independent matching of fixed-order calculations and logarithmic resummations [81, 86], which is done on a case-by-case basis for individual observables [87] up to now. The formalism can be extended to include massive fermions [88]. For processes with initial-state partons, antenna subtrac- 
tion has been fully worked out only to NLO so far [89]. In this case, one encounters two new types of antenna functions, initial-final antenna function with one radiator parton in the initial state, and initial-initial antenna functions with both radiator partons in the initial state. In this work, we derive all NNLO initial-final antenna functions and perform their integration over the appropriately factorized phase space. These functions form part of the full set of antenna functions needed for NNLO calculations of hadron collider processes, and are, together with the already known final-final antenna subtraction terms, sufficient for NNLO calculations of jet observables in deeply inelastic lepton-hadron scattering.

Other approaches to perform NNLO calculations of exclusive observables with initial state partons are the use of sector decomposition and a subtraction method based on the transverse momentum structure of the final state. The sector decomposition algorithm [9093] analytically decomposes both phase space and loop integrals into their Laurent expansion in dimensional regularization, and performs a subsequent numerical computation of the coefficients of this expansion. Using this formalism, NNLO results were obtained for Higgs production [94-96] and vector boson production [97] at hadron colliders. Both reactions were equally computed independently [98, 99], using an NNLO subtraction formalism exploiting the specific transverse momentum structure of these observables [64].

This paper is structured as follows: in section 2, we construct the subtraction terms required at NNLO for initial-final configurations with two unresolved partons at tree-level or one unresolved parton at one loop. They require two new types of antenna functions: tree-level $2 \rightarrow 3$ and one-loop $2 \rightarrow 2$, each with one parton and one off-shell neutral current in the initial state. The phase space mappings relevant to these antenna subtraction terms are discussed in section 3 . The analytic integration of the new initial-final antenna functions is described in section 4 , it proceeds through a reduction to a set of master integrals, which we computed and collect in appendix A. The integrated antenna functions are listed in sections 5 and 6 for the quark-initiated and gluon-initiated antennae. A strong check on these results is provided by the rederivation of NNLO coefficient functions for deep inelastic scattering, which we carry out in section 7 . Finally, we conclude with an outlook in section 8.

\section{Initial-final antenna subtraction at NNLO}

Antenna subtraction of initial-final configurations at NLO is derived in detail in [89]. Subtraction terms in the case of one hard parton in the initial state are built in the same fashion as for the final-final case (formula (2.5) in [65]). We have the following subtraction term associated to a hard radiator parton $i$ with momentum $p$ in the initial state:

$$
\begin{aligned}
\mathrm{d} \hat{\sigma}^{S,(i f)}(p, r)=\mathcal{N} \sum_{m+1} \mathrm{~d} \Phi_{m+1}\left(k_{1}, \ldots, k_{m+1} ; p, r\right) \frac{1}{S_{m+1}} \\
\quad \times \sum_{j} X_{i, j k}^{0}\left|\mathcal{M}_{m}\left(k_{1}, \ldots, K_{K}, \ldots, k_{m+1} ; x p, r\right)\right|^{2} J_{m}^{(m)}\left(k_{1}, \ldots, K_{K}, \ldots, k_{m+1}\right) .
\end{aligned}
$$

The additional momentum $r$ stands for the momentum of the second incoming particle, for example, a virtual boson in DIS, or a second incoming parton in a hadronic collision process. This contribution has to be appropriately convoluted with the parton distribution 
function $f_{i}$. The tree antenna $X_{i, j k}^{0}$, depending only on the original momenta $p, k_{j}$ and $k_{k}$, contains all the configurations in which parton $j$ becomes unresolved. The $m$-parton amplitude depends only on redefined on-shell momenta $k_{1}, \ldots, K_{K}, \ldots$, and on the momentum fraction $x$. In the case where the second incoming particle is a parton, there is an additional convolution with the parton distribution of parton $r$ and corresponding subtraction terms associated with it.

The jet function, $J_{m}^{(m)}$, in (2.1) depends on the momenta $k_{j}$ and $k_{k}$ only through $K_{K}$. Thus, provided a suitable factorization of the phase space, one can perform the integration of the antennae analytically. Due to the hard particle in the initial state, the factorization of phase space is not as straightforward as for final-final antennae.

The phase space can be factorized in an $m$-parton phase space convoluted with a two particle phase space:

$$
\begin{aligned}
\mathrm{d} \Phi_{m+1}\left(k_{1}, \ldots, k_{m+1} ; p, r\right)= & \mathrm{d} \Phi_{m}\left(k_{1}, \ldots, K_{K}, \ldots, k_{m+1} ; x p, r\right) \\
& \times \frac{Q^{2}}{2 \pi} \mathrm{d} \Phi_{2}\left(k_{j}, k_{k} ; p, q\right) \frac{\mathrm{d} x}{x},
\end{aligned}
$$

where $Q^{2}=-q^{2}=-\left(k_{j}+k_{k}-p\right)^{2}$. Replacing the phase space in (2.1), we can explicitly carry out the integration of the antenna factors over the two particle phase space. When combining the integrated subtraction terms with virtual contributions and mass factorization terms, it turns out to be convenient to normalize the integrated antennae as follows

$$
\mathcal{X}_{i, j k}^{0}=\frac{1}{C(\epsilon)} \int \mathrm{d} \Phi_{2} \frac{Q^{2}}{2 \pi} X_{i, j k}^{0},
$$

where

$$
C(\epsilon)=(4 \pi)^{\epsilon} \frac{e^{-\epsilon \gamma_{E}}}{8 \pi^{2}} .
$$

The integrated form of the subtraction term is then

$$
\begin{aligned}
\mathrm{d} \hat{\sigma}^{S,(i f)}(p, r)= & \sum_{m+1} \sum_{j} \frac{\mathcal{N}}{S_{m+1}} \int \frac{\mathrm{d} x}{x} \mathcal{X}_{i, j k}^{0}\left(x, Q^{2}\right) \mathrm{d} \Phi_{m}\left(k_{1}, \ldots, K_{K}, \ldots, k_{m+1} ; x p, r\right) \\
& \times\left|\mathcal{M}_{m}\left(k_{1}, \ldots, K_{K}, \ldots, k_{m+1} ; x p, r\right)\right|^{2} J_{m}^{(m)}\left(k_{1}, \ldots, K_{K}, \ldots, k_{m+1}\right) .
\end{aligned}
$$

Finally, the subtraction term has to be convoluted with the parton distribution functions to give the corresponding contribution to the hadronic cross section. The explicit poles in the integrated form cancel the corresponding ones in the virtual and mass factorization contributions. To carry out the explicit cancellation of poles, it is convenient to recast, by a simple change of variables, the integrated subtraction term, once convoluted with the parton distribution functions (PDFs), in the following form

$$
\begin{aligned}
d \sigma^{S,(i f)}(p, r)= & \sum_{m+1} \sum_{j} \frac{S_{m}}{S_{m+1}} \int \frac{\mathrm{d} \xi_{1}}{\xi_{1}} \int \frac{\mathrm{d} \xi_{2}}{\xi_{2}} \int_{\xi_{1}}^{1} \frac{\mathrm{d} x}{x} f_{i / 1}\left(\frac{\xi_{1}}{x}\right) f_{b / 2}\left(\xi_{2}\right) \\
& \times C(\epsilon) \mathcal{X}_{i, j k}^{0}(x) \mathrm{d} \hat{\sigma}^{B}\left(\xi_{1} H_{1}, \xi_{2} H_{2}\right) .
\end{aligned}
$$

This convolution has already the appropriate structure and mass factorization can be carried out explicitly leaving a finite contribution. The remaining phase space integration, 
implicit in the Born cross section, $\mathrm{d} \hat{\sigma}^{B}$, and the convolutions can be safely done numerically. When considering reactions with only one incoming hadron, the second PDF has to be replaced by a Dirac delta. Reactions with two hadrons will require additional subtractions containing initial-final antennae involving the second parton in the initial state and initial-initial antennae as well. This case is discussed to NLO in [89].

At NNLO, two types of contributions to $m$-jet observables require subtraction: the treelevel $m+2$ parton matrix elements (where one or two partons can become unresolved), and the one-loop $m+1$ parton matrix elements (where one parton can become unresolved). The corresponding subtraction terms are denoted by $\mathrm{d} \sigma_{\mathrm{NNLO}}^{S}$ and $\mathrm{d} \sigma_{\mathrm{NNLO}}^{V S, 1}$. Final-final antenna subtraction terms for both cases are constructed in [65].

In $\mathrm{d} \sigma_{\mathrm{NNLO}}^{S}$, we have to distinguish four different types of unresolved configurations:

(a) One unresolved parton but the experimental observable selects only $m$ jets;

(b) Two colour-connected unresolved partons (colour-connected);

(c) Two unresolved partons that are not colour connected but share a common radiator (almost colour-unconnected);

(d) Two unresolved partons that are well separated from each other in the colour chain (colour-unconnected).

Among those, configuration (a) is properly accounted for by a single tree-level three-parton antenna function like used already at NLO. Configuration (b) requires a tree-level fourparton antenna function (two unresolved partons emitted between a pair of hard partons), while (c) and (d) are accounted for by products of two tree-level three-parton antenna functions. With radiator parton $i$ in the initial state, the subtraction terms for these configurations read:

$$
\begin{aligned}
\mathrm{d} \sigma_{\mathrm{NNLO}}^{S, a}= & \mathcal{N} \sum_{m+2} \mathrm{~d} \Phi_{m+2}\left(k_{1}, \ldots, k_{m+2} ; p, r\right) \frac{1}{S_{m+2}} \\
\times & {\left[\sum_{j} X_{i, j k}^{0}\left|\mathcal{M}_{m+1}\left(k_{1}, \ldots, K_{K}, \ldots, k_{m+2} ; x p, r\right)\right|^{2}\right.} \\
& \left.\times J_{m}^{(m+1)}\left(k_{1}, \ldots, K_{K}, \ldots, k_{m+2}\right)\right], \\
\mathrm{d} \sigma_{\mathrm{NNLO}}^{S, b}= & \mathcal{N} \sum_{m+2} \mathrm{~d} \Phi_{m+2}\left(k_{1}, \ldots, k_{m+2} ; p, r\right) \frac{1}{S_{m+2}} \\
& \times\left[\sum_{j k}\left(X_{i, j k l}^{0}-X_{i, j k}^{0} X_{I, K l}^{0}-X_{j k l}^{0} X_{i, J L}^{0}\right)\right. \\
& \left.\times\left|\mathcal{M} \mathcal{M}_{m}\left(k_{1}, \ldots, K_{L}, \ldots, k_{m+2} ; x p, r\right)\right|^{2} J_{m}^{(m)}\left(k_{1}, \ldots, K_{L}, \ldots, k_{m+2}\right)\right], \\
\mathrm{d} \sigma_{\mathrm{NNLO}}^{S, c 1}= & -\mathcal{N} \sum_{m+2} \mathrm{~d} \Phi_{m+2}\left(k_{1}, \ldots, k_{m+2} ; p, r\right) \frac{1}{S_{m+2}}
\end{aligned}
$$




$$
\begin{aligned}
& \times\left[\sum_{j, l} X_{i, j k}^{0} x_{m l K}^{0}\left|\mathcal{M}_{m}\left(k_{1}, \ldots, K_{K}, K_{M}, \ldots, k_{m+2} ; x p, r\right)\right|^{2}\right. \\
& \times J_{m}^{(m)}\left(k_{1}, \ldots, K_{K}, K_{M}, \ldots, k_{m+2}\right) \\
& +\sum_{j, l} X_{k l m}^{0} x_{i, j K}^{0}\left|\mathcal{M}_{m}\left(k_{1}, \ldots, K_{K}, K_{M}, \ldots, k_{m+2}\right) ; x p, r\right|^{2} \\
& \left.\times J_{m}^{(m)}\left(k_{1}, \ldots, K_{K}, K_{M}, \ldots, k_{m+2}\right)\right], \\
& \mathrm{d} \sigma_{\mathrm{NNLO}}^{S, c 2}=-\mathcal{N} \sum_{m+2} \mathrm{~d} \Phi_{m+2}\left(k_{1}, \ldots, k_{m+2} ; p, r\right) \frac{1}{S_{m+2}} \\
& \times\left[\sum_{j, l} X_{i, j k}^{0} x_{I, l m}^{0}\left|\mathcal{M}_{m}\left(k_{1}, \ldots, K_{K}, K_{M}, \ldots, k_{m+2} ; x p, r\right)\right|^{2}\right. \\
& \times J_{m}^{(m)}\left(k_{1}, \ldots, K_{K}, K_{M}, \ldots, k_{m+2}\right) \\
& +\sum_{j, l} X_{i, l m}^{0} x_{I, j k}^{0}\left|\mathcal{M}_{m}\left(k_{1}, \ldots, K_{K}, K_{M}, \ldots, k_{m+2}\right) ; x p, r\right|^{2} \\
& \left.\times J_{m}^{(m)}\left(k_{1}, \ldots, K_{K}, K_{M}, \ldots, k_{m+2}\right)\right], \\
& \mathrm{d} \sigma_{\mathrm{NNLO}}^{S, d}=-\mathcal{N} \sum_{m+2} \mathrm{~d} \Phi_{m+2}\left(k_{1}, \ldots, k_{m+2} ; p, r\right) \frac{1}{S_{m+2}} \\
& \times\left[\sum_{j, o} X_{i, j k}^{0} X_{n o p}^{0}\left|\mathcal{M}_{m}\left(k_{1}, \ldots, K_{K}, \ldots, K_{N}, K_{P}, \ldots, k_{m+2} ; x p, r\right)\right|^{2}\right. \\
& \left.\times J_{m}^{(m)}\left(k_{1}, \ldots, K_{K}, \ldots, K_{N}, K_{P}, \ldots, k_{m+2}\right)\right] \text {. }
\end{aligned}
$$

As before, the original momenta of the $(m+2)$-parton phase space are denoted by $j, k, \ldots$, while the combined momenta obtained from a phase space mapping are labelled by $J, K, \ldots$ Only the combined momenta appear in the jet function. $x_{a b c}^{0}$ is a three-parton sub-antenna function containing only limits where parton $b$ is unresolved with respect to parton $a$, but not limits where parton $b$ is unresolved with respect to parton $c$. $\mathrm{d} \sigma_{\mathrm{NNLO}}^{S, c 1}$ applies if the common radiator is in the final state, while $\mathrm{d} \sigma_{\mathrm{NNLO}}^{S, c 2}$ applies if the common radiator is in the initial state. The only genuinely new ingredient here is the four-parton initial-final antenna function $X_{i, j k l}^{0}$, which can be obtained by crossing the corresponding final-final antenna functions, and has to be integrated analytically over the appropriate antenna phase space. The resulting integrated antenna function exploits the phase space factorization

$$
\begin{aligned}
\mathrm{d} \Phi_{m+2}\left(k_{1}, \ldots, k_{m+2} ; p, r\right)= & \mathrm{d} \Phi_{m}\left(k_{1}, \ldots, K_{L}, \ldots, k_{m+2} ; x p, r\right) \\
& \times \frac{Q^{2}}{2 \pi} \mathrm{d} \Phi_{3}\left(k_{j}, k_{k}, k_{l} ; p, q\right) \frac{\mathrm{d} x}{x},
\end{aligned}
$$


yielding the integrated antenna

$$
\mathcal{X}_{i, j k l}^{0}=\frac{1}{[C(\epsilon)]^{2}} \int \mathrm{d} \Phi_{3} \frac{Q^{2}}{2 \pi} X_{i, j k l}^{0} .
$$

In all products of two three-parton antenna functions, the analytic integration has to be performed only over the outmost antenna function, yielding the integrated NLO antenna functions in the appropriate (final-final or initial-final) kinematics.

The one-loop single unresolved subtraction term $\mathrm{d} \sigma_{\mathrm{NNLO}}^{V S, 1}$ must account for three types of singular contributions:

(a) Explicit infrared poles of the virtual one-loop $(m+1)$ parton matrix element.

(b) Single unresolved limits of the virtual one-loop $(m+1)$ parton matrix element.

(c) Terms common to both above contributions, which are oversubtracted.

With radiator parton $i$ in the initial state, the subtraction terms for these configurations read:

$$
\begin{aligned}
& \mathrm{d} \sigma_{\mathrm{NNLO}}^{V S, 1, a}=\mathcal{N} \sum_{m+1} \mathrm{~d} \Phi_{m+1}\left(k_{1}, \ldots, k_{m+1} ; p, r\right) \frac{1}{S_{m+1}} \\
& \times\left[\sum_{i k}-\int \frac{\mathrm{d} x}{x} \mathcal{X}_{i, j k}^{0}\left(x,-t_{i k}\right)\left|\mathcal{M}_{m+1}\left(k_{1}, \ldots, k_{k}, \ldots, k_{m+1} ; x p, r\right)\right|^{2}\right. \\
& \left.\quad \times J_{m}^{(m+1)}\left(k_{1}, \ldots, k_{k}, \ldots, k_{m+1}\right)\right] \\
& \mathrm{d} \sigma_{\mathrm{NNLO}}^{V S, 1, b}=\mathcal{N} \sum_{m+1} \mathrm{~d} \Phi_{m+1}\left(k_{1}, \ldots, k_{m+1} ; p, r\right) \frac{1}{S_{m+1}} \\
& \times \sum_{j}\left[X_{i, j k}^{0}\left|\mathcal{M}_{m}^{1}\left(k_{1}, \ldots, \tilde{K}_{K}, \ldots, k_{m+1} ; x p, r\right)\right|^{2} J_{m}^{(m)}\left(k_{1}, \ldots, \tilde{K}_{K}, \ldots, k_{m+1}\right)\right. \\
& \left.\quad+X_{i, j k}^{1}\left|\mathcal{M}_{m}\left(k_{1}, \ldots, \tilde{K}_{K}, \ldots, k_{m+1} ; x p, r\right)\right|^{2} J_{m}^{(m)}\left(k_{1}, \ldots, \tilde{K}_{K}, \ldots, k_{m+1}\right)\right] \\
& \mathrm{d} \sigma_{\mathrm{NNLO}}^{V S, 1, c 1}=\mathcal{N} \sum_{m+1} \mathrm{~d} \Phi_{m+1}\left(k_{1}, \ldots, k_{m+1} ; p, r\right) \frac{1}{S_{m+1}} \\
& \times\left[\sum_{i k} \int \frac{\mathrm{d} x}{x} \mathcal{X}_{i, j k}^{0}\left(x,-t_{i k}\right) \sum_{o} X_{n o p}^{0}\left|\mathcal{M}_{m}\left(k_{1}, \ldots, k_{k}, \ldots, K_{N}, K_{P}, \ldots, k_{m+1} ; x p, r\right)\right|^{2}\right. \\
& \mathrm{d} \sigma_{\mathrm{NNLO}}^{V S, 1, c 2}=\mathcal{N} \sum_{m+1} \mathrm{~d} \Phi_{m+1}\left(k_{1}, \ldots, k_{m+1} ; p, r\right) \frac{1}{S_{m+1}} \\
& \times\left[\sum_{n p} \mathcal{X}_{n o p}^{0}\left(s_{n p}\right) \sum_{j}^{(m)}\left(k_{1}, \ldots, x_{k}^{0}, \ldots, K_{N}, K_{P}, \ldots, k_{m+1}\right)\right] \\
& \\
& \quad\left[\left.\mathcal{M}_{m}\left(k_{1}, \ldots, K_{K}, \ldots, k_{n}, k_{p}, \ldots, k_{m+1} ; x p, r\right)\right|^{2}\right.
\end{aligned}
$$




\begin{tabular}{|lll|}
\hline Quark initiated & tree level & one loop \\
\hline$\underline{\text { quark-quark }}$ & & $A_{q, g q}^{1}, \tilde{A}_{q, g q}^{1}, \hat{A}_{q, g q}^{1}$ \\
$q \rightarrow g q$ & $A_{q, g q}^{0}$ & \\
$q \rightarrow g g q$ & $A_{q, g g q}^{0}, \tilde{A}_{q, g g q}^{0}$ & \\
$q \rightarrow q^{\prime} \bar{q}^{\prime} q$ & $B_{q, q^{\prime} \bar{q}^{\prime} q}^{0}$ & \\
$q^{\prime} \rightarrow q \bar{q} q^{\prime}$ & $B_{q^{\prime}, q \bar{q} q^{\prime}}^{0}$ & \\
$q \rightarrow q \bar{q} q$ & $C_{q, q \bar{q} q}^{0}, C_{\bar{q}, \bar{q} q \bar{q}}^{0}, C_{\bar{q}, q \bar{q} \bar{q}}^{0}$ & \\
\hline$\underline{\text { quark-gluon }}$ & & $D_{q, g g}^{1}, \hat{D}_{q, g g}^{1}$ \\
$q \rightarrow g g$ & $D_{q, g g}^{0}$ & $E_{q, q^{\prime} \bar{q}^{\prime}}^{1}, \tilde{E}_{q, q^{\prime} \bar{q}^{\prime}}^{1}, \hat{E}_{q, q^{\prime} \bar{q}^{\prime}}^{1}$ \\
$q \rightarrow g g g$ & $D_{q, g g g}^{0}$ & $E_{q^{\prime}, q^{\prime} q}^{1}, \tilde{E}_{q^{\prime}, q^{\prime} q}^{1}, \hat{E}_{q^{\prime}, q^{\prime} q}^{1}$ \\
$q \rightarrow q^{\prime} \bar{q}^{\prime}$ & $E_{q, q^{\prime} \bar{q}^{\prime}}^{0}$ & \\
$q \rightarrow q^{\prime} \bar{q}^{\prime} g$ & $E_{q, q^{\prime} \bar{q}^{\prime} g}, \tilde{E}_{q, q^{\prime} \bar{q}^{\prime} g}^{0}$ & \\
$q^{\prime} \rightarrow q^{\prime} q$ & $E_{q^{\prime}, q^{\prime} q}$ & \\
$q^{\prime} \rightarrow q^{\prime} q g$ & $E_{q^{\prime}, q^{\prime} q g}^{0}, \tilde{E}_{q^{\prime}, q^{\prime} q g}^{0}$ & \\
\hline gluon-gluon & & $G_{q, q g}^{1}, \tilde{G}_{q, q g}^{1}, \hat{G}_{q, q g}^{1}$ \\
$q \rightarrow q g$ & $G_{q, q g}^{0}$ & \\
$q \rightarrow q g g$ & $G_{q, q g g}^{0}, \tilde{G}_{q, q g g}^{0}$ & \\
$q \rightarrow q q^{\prime} \bar{q}^{\prime}$ & $H_{q, q q^{\prime} \bar{q}^{\prime}}^{0}$ & \\
\hline
\end{tabular}

Table 1. List of tree level and one loop antenna functions for the initial-final configurations with a quark in the initial state.

$$
\left.\times J_{m}^{(m)}\left(k_{1}, \ldots, K_{K}, \ldots, k_{n}, k_{k}, \ldots, k_{m+1}\right)\right] \text {, }
$$

In here, $X_{i, j k}^{1}$ denotes a one-loop three-parton initial-final antenna function, which is the only new ingredient. These antenna functions can be obtained by crossing from their finalfinal counterparts, listed in [65], and have to be integrated over the appropriate phase space:

$$
\mathcal{X}_{i, j k}^{1}=\frac{1}{C(\epsilon)} \int \mathrm{d} \Phi_{2} \frac{Q^{2}}{2 \pi} X_{i, j k}^{1} .
$$

The subtraction terms $\mathrm{d} \sigma_{\mathrm{NLO}}^{S}, \mathrm{~d} \sigma_{\mathrm{NNLO}}^{S}$ and $\mathrm{d} \sigma_{\mathrm{NNLO}}^{V S, 1}$ require three different types of antenna functions corresponding to the different pairs of hard partons forming the antenna: quark-antiquark, quark-gluon and gluon-gluon antenna functions. We derived these antenna functions [66-68] for final-final kinematics in a systematic manner from physical matrix elements known to possess the correct limits. For the initial-final kinematics, one parton is crossed into the initial state. Special care has to be taken in these crossings, if we 


\begin{tabular}{|lll|}
\hline Gluon initiated & tree level & one loop \\
\hline$\underline{\text { quark-quark }}$ & & \\
$g \rightarrow q \bar{q}$ & $A_{g, q \bar{q}}^{0}$ & $A_{g, q \bar{q}}^{1}, \tilde{A}_{g, q \bar{q}}^{1}, \hat{A}_{g, q \bar{q}}^{1}$ \\
$g \rightarrow g q \bar{q}$ & $A_{g, g q \bar{q}}^{0}, \tilde{A}_{g, g q \bar{q}}^{0}$ & \\
\hline$\underline{\text { quark-gluon }}$ & & \\
$g \rightarrow g g$ & $D_{g, g q}^{0}$ & $D_{g, g q}^{1}, \hat{D}_{g, g q}^{1}$ \\
$g \rightarrow g g q$ & $D_{g, g g q}^{0}, D_{g^{\prime}, g g q}^{0}$ & \\
$g \rightarrow q q^{\prime} \bar{q}^{\prime}$ & $E_{g, q q^{\prime} \bar{q}^{\prime}}, \tilde{E}_{g, q q^{\prime} \bar{q}^{\prime}}^{0}$ & \\
\hline gluon-gluon & $F_{g, g g}^{0}$ & $F_{g, g g}^{1}, \hat{F}_{g, g g}^{1}$ \\
$g \rightarrow g g$ & $F_{g, g g g}^{0}$ & $G_{g, q \bar{q}}^{1}, \tilde{G}_{g, q \bar{q}}^{1}, \hat{G}_{g, q \bar{q}}^{1}$ \\
$g \rightarrow g g g$ & $G_{g, q \bar{q}}^{0}$ & \\
$g \rightarrow q \bar{q}$ & $G_{g, q \bar{q} g}^{0}, \tilde{G}_{g, q \bar{q} g}^{0}$ \\
$g \rightarrow q \bar{q} g$ & & \\
\hline
\end{tabular}

Table 2. List of tree level and one loop antenna functions for the initial-final configurations with a gluon in the initial state.

start from those final-final antenna functions which contain more than one quark/antiquark or more than one gluon in the final state. In the case of more than one quark/antiquark pair of different flavour (final-final antenna functions $B_{4}^{0}, E_{3}^{0}, E_{3}^{1}$-type, $E_{4}^{0}$-type), we have to distinguish the crossing of the primary quark $q$ (which is coupled to the external current) and the secondary quark $q^{\prime}$ (which is not coupled to the external current). The identical flavour antenna function $C_{4}^{0}\left(1_{q}, 3_{q}, 4_{\bar{q}}, 2_{\bar{q}}\right)$ is constructed from the interference of the four-quark amplitudes with the antiquark-momenta interchanged, and it contains only the $\left(3_{q}, 4_{\bar{q}}, 2_{\bar{q}}\right)$ triple collinear limit. Consequently, it is symmetric in the two antiquark momenta, but not in the two quark momenta, and has thus three different crossings: $C_{q, q \bar{q} q}^{0}$ (either antiquark crossed), $C_{\bar{q}, \bar{q} q \bar{q}}^{0}$ (quark $\left(1_{q}\right)$ crossed) and $C_{\bar{q}, q \bar{q} \bar{q}}^{0}$ (quark $\left(3_{q}\right)$ crossed). Crossing one of several gluons into the initial state is unambiguous for most antenna functions owing to their symmetry properties. The only exception is the quark-gluon antenna function $D_{4}^{0}\left(1_{q}, 3_{g}, 4_{g}, 5_{g}\right)$, where gluons $\left(3_{g}\right)$ and $\left(5_{g}\right)$ are colour-connected to the quark, while gluon $\left(4_{g}\right)$ is not. We thus distinguish two crossings, $D_{g, g g q}^{0}$ (gluon $\left(3_{g}\right)$ or $\left(5_{g}\right)$ crossed) and $D_{g^{\prime}, g g q}^{0}$ (gluon $\left(4_{g}\right)$ crossed). We list all NLO and NNLO initial-final antenna functions with an initial state quark in table 1 and with an initial state gluon in table 2 .

It was shown in $[71,72]$ that these antenna subtraction terms result in an oversubtraction of large-angle soft gluon radiation. To correct for this oversubtraction, one introduces the soft antenna function

$$
S_{a j c}=2 \frac{s_{a c}}{s_{a j} s_{c j}}
$$

where $a$ and $c$ label arbitrary hard partons. Those soft factors are associated with an 
antenna phase space mapping $(i, j, k) \rightarrow(I, K)$ (final-final) or $(p, j, k) \rightarrow(x p, K)$ (initialfinal). In contrast to all previous subtraction terms, the hard momenta $a, c$ do not need to be equal to the hard momenta of partons $i, k$ in the antenna phase space - they can be arbitrary on-shell momenta in the initial or final state.

If parton $(a)$ is in the initial state, and $(c, i, j, k)$ are in the final state, the integral of each of the soft antenna function over the antenna phase space can be written as

$$
\begin{aligned}
\mathcal{S}_{a, c ; i k} & =\int \mathrm{d} \Phi_{X_{i j k}} S_{a j c} \\
& =\left(s_{I K}\right)^{-\epsilon} \frac{\Gamma^{2}(1-\epsilon) e^{\epsilon \gamma}}{\Gamma(1-3 \epsilon)}\left(-\frac{2}{\epsilon}\right)\left[-\frac{1}{\epsilon}+\ln \left(x_{a c, I K}\right)+\epsilon \operatorname{Li}_{2}\left(-\frac{1-x_{a c, I K}}{x_{a c, I K}}\right)\right],
\end{aligned}
$$

where we have defined

$$
x_{a c, I K}=\frac{t_{a c} s_{I K}}{\left(t_{a I}+t_{a K}\right)\left(s_{c I}+s_{c K}\right)} .
$$

If parton $(i)$ is in the initial state, while $(a, c, j, k)$ are in the final state, we obtain the following integral:

$$
\begin{aligned}
\mathcal{S}_{a c ; i, k} & =\frac{1}{C(\epsilon)} \int \mathrm{d} \Phi_{2_{j k}} \frac{Q^{2}}{2 \pi} S_{a j c} \\
& =\left(Q^{2}\right)^{-\epsilon} \frac{\Gamma^{2}(1-\epsilon) \Gamma(1+e) e^{\epsilon \gamma}}{\Gamma(1-2 \epsilon)}\left(-\frac{2}{\epsilon}\right) x^{1+2 \epsilon}(1-x)^{-1-2 \epsilon} y_{a c, i K}^{-\epsilon},
\end{aligned}
$$

where we have defined

$$
y_{a c, i K}=\frac{s_{a c} Q^{2}}{\left(s_{a K}+(1-x) s_{a i}\right)\left(s_{c K}+(1-x) s_{c i}\right)} .
$$

\section{$3 \quad$ Phase space mappings}

As discussed above, the construction of subtraction terms requires mapping the original set of momenta onto a reduced set. The mappings interpolate between the different soft and collinear limits which the subtraction term regulates. Appropriate mappings for the initial-final configurations, both for single and double unresolved configurations have been discussed in [89], and are only briefly summarized here.

The proper subtraction of infrared singularities requires that the momentum mapping satisfy

$$
\begin{array}{lll}
x p \rightarrow p, & K_{K} \rightarrow k_{k} & \text { when } j \text { becomes soft, } \\
x p \rightarrow p, & K_{K} \rightarrow k_{j}+k_{k} & \text { when } j \text { becomes collinear with } k, \\
x p \rightarrow p-k_{j}, & K_{K} \rightarrow k_{k} & \text { when } j \text { becomes collinear with } i .
\end{array}
$$

In this way, infrared singularities are subtracted locally, except for angular correlations, before convoluting with the parton distributions. That is, matrix elements and subtraction terms are convoluted together with PDFs. In addition, the redefined momentum, $K_{K}$, 
must be on shell and momentum must be conserved, $p-k_{j}-k_{k}=x p-K_{K}$, for the phase space to factorize as above. This is accomplished by:

$$
\begin{gathered}
x=\frac{s_{1 j}+s_{1 k}-s_{j k}}{s_{1 j}+s_{1 k}}, \\
K_{K}=k_{j}+k_{k}-(1-x) p,
\end{gathered}
$$

where $s_{1 j}=\left(p-k_{j}\right)^{2}$, etc. If parton $j$ becomes soft or collinear to parton $k, x \rightarrow 1$. If parton $j$ becomes collinear with the initial state parton $i, x=1-z$ with $z$ the fraction of the momentum $p$ carried by parton $j$.

This mapping is easily generalized to deal with more than one parton becoming unresolved. As explained above, the building blocks for the double real radiation in the initial-final situation are colour-ordered four-parton antenna functions $X_{i, j k l}$, with one radiator parton $i$ (with momentum $p$ ) in the initial state, two unresolved partons $j, k$ and one radiator parton $l$ in the final state:

$$
\begin{aligned}
x & =\frac{s_{1 j}+s_{1 k}+s_{1 l}-s_{j k}-s_{j l}-s_{k l}}{s_{1 j}+s_{1 k}+s_{1 l}}, \\
K_{L} & =k_{j}+k_{k}+k_{l}-(1-x) p
\end{aligned}
$$

where $k_{j}, k_{k}$ and $k_{l}$ are the three final-state momenta involved in the subtraction term. It satisfies the appropriate limits in all double singular configurations:

1. $j$ and $k$ soft: $x \rightarrow 1, K_{L} \rightarrow k_{l}$,

2. $j$ soft and $k_{k} \| k_{l}: x \rightarrow 1, K_{L} \rightarrow k_{k}+k_{l}$,

3. $k_{j}=z p \| p$ and $k_{k}$ soft: $x \rightarrow 1-z, K_{L} \rightarrow k_{l}$,

4. $k_{j}=z p \| p$ and $k_{k} \| k_{l}: x \rightarrow 1-z, K_{L} \rightarrow k_{k}+k_{l}$,

5. $k_{j}\left\|k_{k}\right\| k_{l}: x \rightarrow 1, K_{L} \rightarrow k_{j}+k_{k}+k_{l}$,

6. $k_{j}+k_{k}=z p \| p: x \rightarrow 1-z, K_{L} \rightarrow k_{l}$,

where partons $j$ and $k$ can be interchanged in all cases.

The construction of NNLO antenna subtraction terms requires moreover that all single unresolved limits of the four-parton antenna function $X_{i, j k l}$ have to be subtracted, (2.8), such that the resulting subtraction term is active only in its double unresolved limits. A systematic subtraction of these single unresolved limits by products of two three-parton antenna functions can be performed only if the NNLO phase space mapping turns into an NLO phase space mapping in its single unresolved limits.

In the limits where parton $j$ becomes unresolved, we denote the parameters of the reduced NLO phase space mapping (3.2) by $x^{\prime}$ and $K_{L}^{\prime}$. We find for (3.3):

1. $j$ becomes soft:

$$
x \rightarrow \frac{s_{1 k}+s_{1 l}-s_{k l}}{s_{1 k}+s_{1 l}}=x^{\prime}, \quad K_{L} \rightarrow k_{k}+k_{l}-(1-x) p=K_{L}^{\prime} .
$$


2. $k_{j} \| k_{k}, k_{j}+k_{k}=K_{K}$ :

$$
x \rightarrow \frac{s_{1 K}+s_{1 l}-s_{K l}}{s_{1 K}+s_{1 l}}=x^{\prime}, \quad K_{L} \rightarrow k_{K}+k_{l}-(1-x) p=K_{L}^{\prime} .
$$

3. $k_{j}=z p \| p$ :

$$
x \rightarrow \frac{(1-z)\left(s_{1 k}+s_{1 l}\right)-s_{k l}}{s_{1 k}+s_{1 l}}=(1-z) x^{\prime}, \quad K_{L} \rightarrow k_{k}+k_{l}-\left(1-x^{\prime}\right)(1-z) p=K_{L}^{\prime} .
$$

It can be seen that in the first two limits, the NLO mapping involves the original incoming momentum $p$, while in the last limit (initial state collinear emission), it involves the rescaled incoming momentum $(1-z) p$. To subtract all three single unresolved limits of parton $j$ between emitter partons $i$ and $k$ from $X_{i, j k l}$, one needs to subtract from it the product of two three-parton antenna functions $X_{i, j k} \cdot X_{I, K l}$. The phase space mapping relevant to these terms is the iteration of two NLO phase space mappings. Analytical integration of terms with this mapping is required only over the phase space appropriate to the first antenna function.

Equally, parton $k$ can become unresolved. Expressing the reduced NLO phase space mapping by $x^{\prime \prime}$ and $K_{L}^{\prime \prime}$. We find for (3.3):

1. $k$ becomes soft:

$$
x \rightarrow \frac{s_{1 j}+s_{1 l}-s_{j l}}{s_{1 j}+s_{1 l}}=x^{\prime \prime}, \quad K_{L} \rightarrow k_{j}+k_{l}-(1-x) p=K_{L}^{\prime \prime} .
$$

2. $k_{k} \| k_{j}, k_{j}+k_{k}=K_{K}$ :

$$
x \rightarrow \frac{s_{1 K}+s_{1 l}-s_{K l}}{s_{1 K}+s_{1 l}}=x^{\prime \prime}, \quad K_{L} \rightarrow k_{K}+k_{l}-(1-x) p=K_{L}^{\prime \prime} .
$$

3. $k_{k} \| k_{l}, k_{l}+k_{k}=K_{K}$ :

$$
x \rightarrow \frac{s_{1 K}+s_{1 j}-s_{K j}}{s_{1 K}+s_{1 j}}=x^{\prime \prime}, \quad K_{L} \rightarrow k_{K}+k_{j}-(1-x) p=K_{L}^{\prime \prime} .
$$

In all limits, the reduced NLO mapping involves the original incoming momentum $p$. Consequently, the three single unresolved limits of parton $k$ between emitter partons $j$ and $l$ can be subtracted from $X_{i, j k l}$ by a product of a final-final and an initial-final three-parton antenna function $X_{j k l} \cdot X_{i, J L}$. The phase space mapping relevant to these terms is the product of an NLO final-final phase space mapping with an initial-final mapping. Integration of the final-final antenna phase space yields a constant, not involving an extra convolution.

\section{Integration of initial-final antenna functions at NNLO}

The initial-final antenna functions all have the scattering kinematics

$$
q+p_{i} \rightarrow p_{1}+p_{2}\left(+p_{3}\right)
$$


where

$$
q^{2}=-Q^{2}<0, \quad p_{i}^{2}=0, z=\frac{Q^{2}}{2 q \cdot p_{i}}, \quad p_{1}^{2}=p_{2}^{2}=p_{3}^{2}=0,
$$

and $p_{3}$ is present only for the NNLO real radiation antenna functions. Consequently, integration over the final-state two-parton or three-parton phase space yields a result which depends only on $Q^{2}$ and $z$. From dimensional counting, one can immediately conclude that the dependence on $Q^{2}$ is only multiplicative, according to the mass dimension of the integral.

The NNLO double real radiation antenna functions $X_{i, j k l}^{0}$ have to be integrated over the inclusive three-parton final state phase space. The NNLO one-loop single real radiation antenna functions $X_{i, j k}^{1}$ are integrated over the inclusive two-parton final state phase space, and over the loop momentum. For both types of integration, we employ the by-now standard technique of reduction to master integrals. The master integrals are then computed from their differential equations.

To perform the reduction, we first express all phase space integrals as loop integrals with cut propagators [100]. Consequently, all NNLO integrals are expressed as cuts of twoloop four-point functions with two off-shell legs in forward scattering kinematics. Using integration-by-parts (IBP, [101, 102]) and Lorentz invariance (LI, [103]) identities among the integrals of any given topology, the large number of different integrals can be expressed in terms of a small number of master-integrals. This reduction is performed iteratively, based on the lexicographic ordering of the integrals, expressed by the Laporta algorithm [104].

After carrying out the reduction, one finds nine master integrals for the NNLO double real radiation antenna functions, described in section 4.1 below, and listed in appendix A.1. For the NNLO one-loop single real radiation antennae, one finds six master integrals, which we describe in section 4.2 and list in appendix A.2.

We computed these integrals both directly and by using the differential equation technique [103, 105-111]. To derive the differential equations for each master integral, we employ

$$
\begin{aligned}
Q^{2} \frac{\partial}{\partial Q^{2}} & =\frac{1}{2} q^{\mu} \frac{\partial}{\partial q^{\mu}}+\frac{1}{2} p_{i}^{\mu} \frac{\partial}{\partial p_{i}^{\mu}} \\
z \frac{\partial}{\partial z} & =-p_{i}^{\mu} \frac{\partial}{\partial p_{i}^{\mu}}
\end{aligned}
$$

to carry out the differentiations at the integrand level. The boundary conditions required for the solution of the differential equations are either obtained from self-consistency conditions on the integrals, or by explicit evaluation. The explicit evaluation is very similar to the evaluation of inclusive four-point phase space integrals, described in [112].

Some of the more involved master integrals can be related to phase space integrals computed by Zijlstra and van Neerven in the context of the NNLO corrections to deep inelastic structure functions $[113,114]$. Where appropriate, we compared our results to the expressions in the appendix of [113, 114], finding full agreement. Explicit expressions for all master integrals are listed in appendix A.1-A.2. 
All master integrals contain multiplicative factors of the form $(1-z)^{-\epsilon}$ or $(1-z)^{-2 \epsilon}$, which regulate soft endpoint singularities in initial state convolution integrals. These factors must be left as such in the master integrals, and can be expanded in the form of distributions

$$
(1-z)^{-1-\epsilon}=-\frac{1}{\epsilon} \delta(1-z)+\sum_{n} \frac{(-\epsilon)^{n}}{n !} \mathcal{D}_{n}(z)
$$

with

$$
\mathcal{D}_{n}(z)=\left(\frac{\ln ^{n}(1-z)}{1-z}\right)_{+},
$$

only after being inserted into the integrated antenna functions. All other terms in the master integrals can be expanded, yielding Harmonic Polylogarithms (HPLs, [115-118]) of argument $z$.

\subsection{Tree-level $2 \rightarrow 3$ antenna functions}

For the tree-level $2 \rightarrow 3$ antenna functions, we have the 'DIS-like' process

$$
q+p_{i} \rightarrow p_{1}+p_{2}+p_{3}
$$

There are 12 propagators, including the three that are cut in the phase space integration $\left(D_{10}, D_{11}, D_{12}\right)$ :

$$
\begin{aligned}
D_{1} & =\left(p_{i}-p_{1}\right)^{2}, \\
D_{2} & =\left(q-p_{1}\right)^{2}, \\
D_{3} & =\left(p_{2}+p_{3}\right)^{2}, \\
D_{4} & =\left(p_{i}-p_{2}\right)^{2}, \\
D_{5} & =\left(q-p_{2}\right)^{2}, \\
D_{6} & =\left(p_{1}+p_{3}\right)^{2}, \\
D_{7} & =\left(p_{i}-p_{3}\right)^{2}, \\
D_{8} & =\left(q-p_{3}\right)^{2}, \\
D_{9} & =\left(p_{1}+p_{2}\right)^{2}, \\
D_{10} & =p_{1}^{2}, \\
D_{11} & =p_{2}^{2}, \\
D_{12} & =p_{3}^{2} .
\end{aligned}
$$

To perform the reduction to master integrals, we impose momentum conservation $p_{3}=$ $q+p_{i}-p_{1}-p_{2}$, set $p_{i}^{2}=0, q^{2}=-Q^{2}$ and drop any integral where $D_{10}, D_{11}$ and $D_{12}$ are not in the denominator. After labelling the inclusive phase space integral as $I[0]$, the convention for naming the master integrals follows the labelling of the numerators, i.e.

$$
I[1,2,5]=\int \frac{\left[\mathrm{d} p_{1}\right]\left[\mathrm{d} p_{2}\right]\left[\mathrm{d} p_{3}\right]}{D_{1} D_{2} D_{5}} \delta^{d}\left(q+p_{i}-p_{1}-p_{2}-p_{3}\right)
$$

where

$$
[\mathrm{d} p]=\frac{\mathrm{d}^{d} p}{(2 \pi)^{d}} \delta^{+}\left(p^{2}\right) .
$$




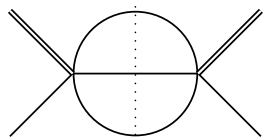

$\mathbf{I}[\mathbf{0}]$

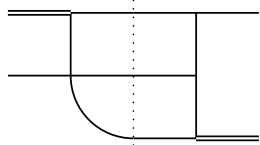

$\mathrm{I}[\mathbf{1}, \mathbf{2}, \mathbf{5}]$

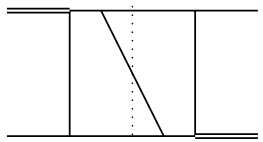

$\mathrm{I}[\mathbf{1}, \mathbf{3}, \mathbf{4}, \mathbf{6}]$

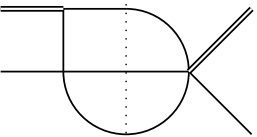

$\mathbf{I}[2]$

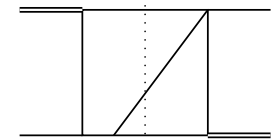

$\mathbf{I}[2,3,5]$

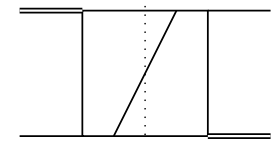

$\mathrm{I}[2,3,5,6]$

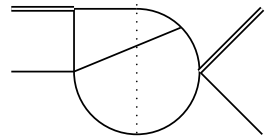

$\mathbf{I}[2,6]$

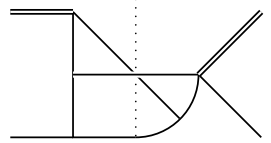

$\mathrm{I}[2,4,9]$

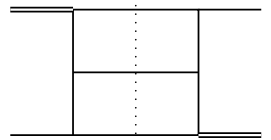

$\mathrm{I}[1,2,4,5]$

Figure 1. Master integrals for the phase space integration of the tree level initial-final antennae at NNLO. The double line in the external states represents the off-shell momentum, $q$ with $q^{2}=-Q^{2}$, the single one is the incoming parton. All internal lines are massless. The cut propagators are the ones intersected by the dotted line.

\begin{tabular}{|c|c|c|c|}
\hline master & deepest pole & behaviour at $x=1$ & known to order \\
\hline$I[0]$ & $\epsilon^{0}$ & $(1-x)^{1-2 \epsilon}$ & all \\
$I[2]$ & $\epsilon^{0}$ & $(1-x)^{-2 \epsilon}$ & all, $\epsilon^{5}$ \\
$I[2,6]$ & $\epsilon^{-1}$ & $(1-x)^{-2 \epsilon}$ & all, $\epsilon^{5}$ \\
$I[1,2,5]$ & $\epsilon^{-2}$ & $(1-x)^{-2 \epsilon}$ & $\epsilon^{3}$ \\
$I[2,3,5]$ & $\epsilon^{-2}$ & $(1-x)^{-1-2 \epsilon}$ & $\epsilon^{3}$ \\
$I[2,4,9]$ & $\epsilon^{-3}$ & $(1-x)^{-2 \epsilon}$ & $\epsilon^{3}$ \\
$I[1,3,4,6]$ & $\epsilon^{-3}$ & $(1-x)^{-1-2 \epsilon}$ & all, $\epsilon^{5}$ \\
$I[2,3,5,6]$ & $\epsilon^{-3}$ & $(1-x)^{-1-2 \epsilon}$ & $\epsilon^{1}$ \\
$I[1,2,4,5]$ & $\epsilon^{-2}$ & $(1-x)^{-2 \epsilon}$ & $\epsilon^{1}$ \\
\hline
\end{tabular}

Table 3. Summary of the main properties of the three particles phase space master integrals

When squaring the $2 \rightarrow 3$ antennae, we find at most 4 propagators, plus the 3 cut ones. All the integrals can be reduced to the set of 9 master integrals shown in figure 1 .

All the masters, except $I[1,2,4,5]$, have been computed by direct integration and by the differential equations method, supplemented, where necessary, by a direct calculation at $x=1$ after factorizing the leading singularity. The $x=1$ boundary conditions for $I[1,3,4,6]$ and $I[2,3,5,6]$ were checked numerically using sector decomposition [90-93]. $I[1,2,4,5]$ has been computed only using the differential equations method. The master integrals $I[1,2,5], I[2,3,5], I[2,3,5,6]$ and $I[1,2,4,5]$ agree up to order $\epsilon^{0}$ with the results in $[113,114]$. We summarize in table 3 some of the properties of the master integrals. 


\begin{tabular}{|c|c|c|c|c|}
\hline & Topology 1 & Topology 2 & Topology 3 & Topology 4 \\
\hline$D_{1}$ & $k^{2}$ & $k^{2}$ & $k^{2}$ & $k^{2}$ \\
$D_{2}$ & $\left(k+p_{i}\right)^{2}$ & $\left(k+p_{i}\right)^{2}$ & $\left(k+p_{i}\right)^{2}$ & $\left(k+p_{i}\right)^{2}$ \\
$D_{3}$ & $\left(k+p_{i}-p_{1}\right)^{2}$ & $\left(k+p_{i}-p_{1}\right)^{2}$ & $\left(k+p_{i}-p_{1}\right)^{2}$ & $\left(k+p_{i}-p_{1}\right)^{2}$ \\
$D_{4}$ & $(k-q)^{2}$ & $(k-q)^{2}$ & $\left(k+p_{2}\right)^{2}$ & $\left(k+p_{2}\right)^{2}$ \\
$D_{5}$ & $\left(p_{i}-p_{i}\right)^{2}$ & $\left(q-p_{1}\right)^{2}$ & $\left(p_{i}-p_{1}\right)^{2}$ & $\left(q-p_{1}\right)^{2}$ \\
$D_{6}$ & $p_{1}^{2}$ & $p_{1}^{2}$ & $p_{1}^{2}$ & $p_{1}^{2}$ \\
$D_{7}$ & $p_{2}^{2}$ & $p_{2}^{2}$ & $p_{2}^{2}$ & $p_{2}^{2}$ \\
\hline
\end{tabular}

Table 4. Definition of the topologies for the combined phase space and loop integration.

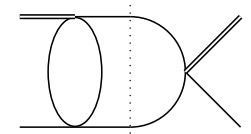

$\mathrm{V}[1,3$

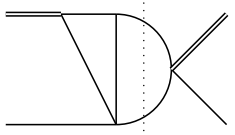

$\mathrm{V}[\mathbf{1}, \mathbf{3}, 4]$

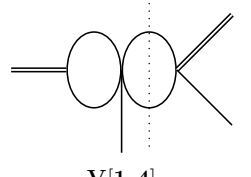

$\mathrm{V}[\mathbf{1}, \mathbf{4}]$

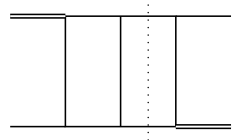

$\mathrm{V}[1,2,3,4,5$

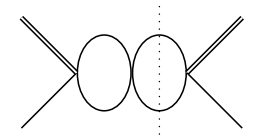

$\mathrm{V}[2,4]$

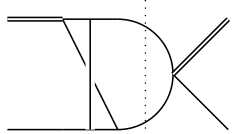

$\mathrm{C}[1,2,3,4]$

Figure 2. Master integrals for the loop plus phase space integration of the one loop initial-final antennae at NNLO. The double line in the external states represents the off-shell momentum, $q$ with $q^{2}=-Q^{2}$, the single one is the incoming parton. All internal lines are massless. The cut propagators are the ones intersected by the dotted line.

\subsection{One-loop $2 \rightarrow 2$ antenna functions}

When interfering the one loop $2 \rightarrow 2$ antennae with the corresponding tree level ones, we can combine the loop and phase space integrations. The partonic process in this case is

$$
q+p_{i} \rightarrow p_{1}+p_{2}
$$

Denoting the loop momentum by $k$, we can identify four topologies, two planar (Topology 1 and 2) and two non-planar (Topology 3 and 4). The topologies are defined in table 4 . Topologies 1 and 2 only differ in the propagator $D_{5}$, as is also the case for Topologies 3 and 4. Subtopologies of the non-planar integrals can be expressed by the planar topologies.

All the resulting integrals can again be reduced to a small set of masters. In this case we find only six of them. They are shown in figure 2. The notation for the master integrals follows the propagator definitions of Topology 2 in the planar case (denoted by $V$ ) and of Topology 3 in the non-planar case (denoted by $C$ ).

The one loop plus phase-space master integrals have been computed using differential equations in external invariants, together with a direct calculation at $x=1$ after factorizing the leading singularity at $x=1$. Integrals $V[1,2,3,4], V[1,2,3,4,5]$ and $C[1,2,3,4]$ have 


\begin{tabular}{|c|c|c|c|}
\hline master & deepest pole & behaviour at $x=1$ & known to order \\
\hline$V[1,3]$ & $\epsilon^{-1}$ & $(1-x)^{-\epsilon}$ & all, $\epsilon^{5}$ \\
$V[1,4]$ & $\epsilon^{-1}$ & $(1-x)^{-\epsilon}$ & all, $\epsilon^{5}$ \\
$V[2,4]$ & $\epsilon^{-1}$ & $(1-x)^{-2 \epsilon}$ & all, $\epsilon^{5}$ \\
$V[1,3,4]$ & $\epsilon^{-1}$ & $(1-x)^{-\epsilon}$ & $\epsilon^{3}$ \\
$V[1,2,3,4,5]$ & $\epsilon^{-3}$ & $(1-x)^{-1-2 \epsilon}$ & $\epsilon^{3}$ \\
$C[1,2,3,4]$ & $\epsilon^{-3}$ & $(1-x)^{-\epsilon}$ & $\epsilon^{3}$ \\
\hline
\end{tabular}

Table 5. Summary of the main properties of the one loop plus two particles phase space master integrals

been checked, up to order $\epsilon^{0}$, against a direct analytic calculation. We summarize in table 5 some of the properties of the master integrals. Explicit expressions for the master integrals are listed in appendix A.2.

We always integrate the unrenormalized $2 \rightarrow 2$ one-loop squared matrix elements, divided by a normalization factor $C(\epsilon)$, relevant to a particular antenna function, which we denote as $X_{i, j k}^{1, U}$. The antenna function is obtained after renormalization and subtraction of the corresponding tree-level antenna function multiplied with the one-loop correction to the hard radiator pair. Renormalization of the one-loop antenna functions is always carried out in the $\overline{\mathrm{MS}}$-scheme at fixed renormalization scale $\mu^{2}=Q^{2}$. It amounts to a renormalization of the strong coupling constant and (in the case of the quark-gluon and gluon-gluon antenna functions) to a renormalization of the effective operators used to couple an external current to the partonic radiators. The relation between renormalized and unrenormalized one-loop squared matrix elements is as follows:

$$
\begin{aligned}
& X_{i, j k}^{1, R}=X_{i, j k}^{1, U}-\frac{b_{0}}{\epsilon} X_{i, j k}^{0}-\frac{\eta_{0}}{\epsilon} X_{i, j k}^{0}, \\
& \tilde{X}_{i, j k}^{1, R}=\tilde{X}_{i, j k}^{1, U}, \\
& \hat{X}_{i, j k}^{1, R}=X_{i, j k}^{1, U}-\frac{b_{0, F}}{\epsilon} X_{i, j k}^{0}-\frac{\eta_{0, F}}{\epsilon} X_{i, j k}^{0},
\end{aligned}
$$

where

$$
b_{0}=\frac{11}{6}, \quad b_{0, F}=-\frac{1}{3}
$$

are the colour-ordered coefficients of the one-loop QCD $\beta$-function:

$$
\beta_{0}=b_{0} N+b_{0, F} N_{F} .
$$

The renormalization constants for the effective operators are

$$
\begin{array}{lll}
\eta_{0}=0, & \eta_{0, F}=0 & \text { for } X=A, \\
\eta_{0}=b_{0}+\frac{3}{2}, & \eta_{0, F}=b_{0, F} & \text { for } X=D, E, \\
\eta_{0}=2 b_{0}, & \eta_{0, F}=2 b_{0, F} & \text { for } X=F, G .
\end{array}
$$


The one-loop antenna functions are obtained from the renormalized one-loop squared matrix elements by subtracting from them the product of the tree-level antenna function with the the virtual one-loop hard radiator vertex correction [29-33, 65]:

$$
\begin{aligned}
& X_{i, j k}^{1}=X_{i, j k}^{1, R}-\operatorname{Re}\left((-1)^{-\epsilon} \mathcal{X}_{2}^{1}\right) X_{i, j k}^{0}, \\
& \tilde{X}_{i, j k}^{1}=\tilde{X}_{i, j k}^{1, R}-\operatorname{Re}\left((-1)^{-\epsilon} \tilde{\mathcal{X}}_{2}^{1}\right) X_{i, j k}^{0}, \\
& \hat{X}_{i, j k}^{1}=X_{i, j k}^{1, U}-\operatorname{Re}\left((-1)^{-\epsilon} \hat{\mathcal{X}}_{2}^{1}\right) X_{i, j k}^{0} .
\end{aligned}
$$

The one-loop corrections to the hard radiator vertex are listed in [65] for $\mathcal{A}_{2}^{1}, \mathcal{D}_{2}^{1}, \hat{\mathcal{D}}_{2}^{1}, \mathcal{F}_{2}^{1}$ and $\hat{\mathcal{F}}_{2}^{1}$. From these, the remaining functions follow:

$$
\begin{array}{rlrl}
\tilde{\mathcal{A}}_{2}^{1} & =\mathcal{A}_{2}^{1}, & \hat{\mathcal{A}}_{2}^{1}=0, & \\
\tilde{\mathcal{D}}_{2}^{1}=0, & & \\
\mathcal{E}_{2}^{1}=\mathcal{D}_{2}^{1} & \tilde{\mathcal{E}}_{2}^{1}=0, & \hat{\mathcal{E}}_{2}^{1}=\hat{\mathcal{D}}_{2}^{1}, \\
\tilde{\mathcal{F}}_{2}^{1}=0, & & \\
\mathcal{G}_{2}^{1}=\mathcal{F}_{2}^{1} & \tilde{\mathcal{G}}_{2}^{1}=0, & \hat{\mathcal{G}}_{2}^{1}=\hat{\mathcal{F}}_{2}^{1} .
\end{array}
$$

\section{Quark initiated antennae}

The quark-initiated initial-final antenna functions are obtained from the final-final antenna functions by crossing a quark into the initial state. Their unintegrated analytical expressions are obtained by a pure kinematical crossing from the final-final expressions listed in [65], with no extra symmetry factors or overall signs applied, and no further decompositions into sub-antennae. This procedure differs from the approach used in our previous work on initial-final antenna functions at NLO [89]. In the application of the antenna subtraction formalism to the calculation of NNLO corrections to $e^{+} e^{-} \rightarrow 3 j[69,70]$, it turned out that several decompositions can be circumvented by an appropriate symmetrization of the real radiation matrix elements in the process under consideration, and that the remaining required decompositions can be derived on a case-by-case basis, often not along the lines of $[65,89]$. A systematic decomposition would start from a specific process under consideration, and is beyond the scope of the present study.

The virtual one-loop antenna functions are obtained from the final-final one-loop antenna functions [65], where some attention has to be paid to the correct analytic continuation of the polylogarithmic functions. In the initial-final kinematics, the relevant final state phase space consists of three different Riemann sheets [119-126], which have to be patched together correctly.

All quark-initiated initial-final antenna functions are listed in table 1. We summarize their integrated forms in the following.

\subsection{Quark-quark antennae}

The next-to-leading order initial-final antenna functions were already integrated in [89] through to finite terms of order $\epsilon^{0}$. Applying these antenna functions in an NNLO calculation, one also needs their subleading terms through to $\epsilon^{2}$. To this order, the quark-initiated 
quark-antiquark antenna function is:

$$
\begin{aligned}
& \mathcal{A}_{q, q q}^{0}= \\
& +\frac{1}{\epsilon^{2}}[\delta(1-z)] \\
& +\frac{1}{\epsilon}\left[\frac{1}{2}+\frac{3}{4} \delta(1-z)-\mathcal{D}_{0}(z)+\frac{z}{2}\right] \\
& +\left[\frac{3}{2}+\delta(1-z)\left(\frac{7}{4}-\frac{\pi^{2}}{4}\right)-\frac{3}{4} \mathcal{D}_{0}(z)+\mathcal{D}_{1}(z)+\frac{1}{2} \mathrm{H}(0 ; z)-\frac{1}{1-z} \mathrm{H}(0 ; z)+\frac{1}{2} \mathrm{H}(1 ; z)\right. \\
& \left.+z\left(-\frac{1}{2}+\frac{1}{2} \mathrm{H}(0 ; z)+\frac{1}{2} \mathrm{H}(1 ; z)\right)\right] \\
& +\epsilon\left[3-\frac{\pi^{2}}{8}+\delta(1-z)\left(\frac{7}{2}-\frac{3 \pi^{2}}{16}-\frac{7 \zeta_{3}}{3}\right)+\left(-\frac{7}{4}+\frac{\pi^{2}}{4}\right) \mathcal{D}_{0}(z)+\frac{3}{4} \mathcal{D}_{1}(z)-\frac{1}{2} \mathcal{D}_{2}(z)+\frac{3}{2} \mathrm{H}(0 ; z)\right. \\
& +\frac{3}{2} \mathrm{H}(1 ; z)+\frac{1}{2} \mathrm{H}(0,0 ; z)+\frac{1}{2} \mathrm{H}(0,1 ; z)+\frac{1}{2} \mathrm{H}(1,0 ; z)+\frac{1}{2} \mathrm{H}(1,1 ; z)+\frac{1}{1-z}\left(-\frac{3}{4} \mathrm{H}(0 ; z)\right. \\
& -\mathrm{H}(0,0 ; z)-\mathrm{H}(0,1 ; z)-\mathrm{H}(1,0 ; z))+z\left(-\frac{\pi^{2}}{8}-\frac{1}{2} \mathrm{H}(0 ; z)-\frac{1}{2} \mathrm{H}(1 ; z)+\frac{1}{2} \mathrm{H}(0,0 ; z)\right. \\
& \left.\left.+\frac{1}{2} \mathrm{H}(0,1 ; z)+\frac{1}{2} \mathrm{H}(1,0 ; z)+\frac{1}{2} \mathrm{H}(1,1 ; z)\right)\right] \\
& +\epsilon^{2}\left[6-\frac{3 \pi^{2}}{8}-\frac{7 \zeta_{3}}{6}+\delta(1-z)\left(7-\frac{7 \pi^{2}}{16}-\frac{\pi^{4}}{96}-\frac{7 \zeta_{3}}{4}\right)+\mathcal{D}_{0}(z)\left(-\frac{7}{2}+\frac{3 \pi^{2}}{16}+\frac{7 \zeta_{3}}{3}\right)\right. \\
& +\left(\frac{7}{4}-\frac{\pi^{2}}{4}\right) \mathcal{D}_{1}(z)-\frac{3}{8} \mathcal{D}_{2}(z)+\frac{1}{6} \mathcal{D}_{3}(z)+3 \mathrm{H}(0 ; z)-\frac{1}{8} \pi^{2} \mathrm{H}(0 ; z)+3 \mathrm{H}(1 ; z)-\frac{1}{8} \pi^{2} \mathrm{H}(1 ; z) \\
& +\frac{3}{2} \mathrm{H}(0,0 ; z)+\frac{3}{2} \mathrm{H}(0,1 ; z)+\frac{3}{2} \mathrm{H}(1,0 ; z)+\frac{3}{2} \mathrm{H}(1,1 ; z)+\frac{1}{2} \mathrm{H}(0,0,0 ; z)+\frac{1}{2} \mathrm{H}(0,0,1 ; z) \\
& +\frac{1}{2} \mathrm{H}(0,1,0 ; z)+\frac{1}{2} \mathrm{H}(0,1,1 ; z)+\frac{1}{2} \mathrm{H}(1,0,0 ; z)+\frac{1}{2} \mathrm{H}(1,0,1 ; z)+\frac{1}{2} \mathrm{H}(1,1,0 ; z) \\
& +\frac{1}{2} \mathrm{H}(1,1,1 ; z)+\frac{1}{1-z}\left(-\frac{7}{4} \mathrm{H}(0 ; z)+\frac{1}{4} \pi^{2} \mathrm{H}(0 ; z)-\frac{3}{4} \mathrm{H}(0,0 ; z)-\frac{3}{4} \mathrm{H}(0,1 ; z)\right. \\
& -\frac{3}{4} \mathrm{H}(1,0 ; z)-\mathrm{H}(0,0,0 ; z)-\mathrm{H}(0,0,1 ; z)-\mathrm{H}(0,1,0 ; z)-\mathrm{H}(0,1,1 ; z)-\mathrm{H}(1,0,0 ; z) \\
& -\mathrm{H}(1,0,1 ; z)-\mathrm{H}(1,1,0 ; z))+z\left(\frac{\pi^{2}}{8}-\frac{1}{8} \pi^{2} \mathrm{H}(0 ; z)-\frac{1}{8} \pi^{2} \mathrm{H}(1 ; z)-\frac{1}{2} \mathrm{H}(0,0 ; z)\right. \\
& -\frac{1}{2} \mathrm{H}(0,1 ; z)-\frac{1}{2} \mathrm{H}(1,0 ; z)-\frac{1}{2} \mathrm{H}(1,1 ; z)+\frac{1}{2} \mathrm{H}(0,0,0 ; z)+\frac{1}{2} \mathrm{H}(0,0,1 ; z)+\frac{1}{2} \mathrm{H}(0,1,0 ; z) \\
& \left.\left.+\frac{1}{2} \mathrm{H}(0,1,1 ; z)+\frac{1}{2} \mathrm{H}(1,0,0 ; z)+\frac{1}{2} \mathrm{H}(1,0,1 ; z)+\frac{1}{2} \mathrm{H}(1,1,0 ; z)+\frac{1}{2} \mathrm{H}(1,1,1 ; z)-\frac{7 \zeta_{3}}{6}\right)\right] \\
& +\mathcal{O}\left(\epsilon^{3}\right) \text {. }
\end{aligned}
$$

At NNLO, the quark-initiated initial-final quark-antiquark double real radiation antenna are the crossings of the corresponding final-final quark-antiquark antenna functions. Depending on the unresolved particles, they are of the $A_{4}^{0}$-type (two unresolved gluons), $B_{4}^{0}$ type (unresolved secondary quark pair of different flavour) and $C_{4}^{0}$-type (pure interference contribution for secondary quark pair of identical flavour).

The integrated form of the initial-final $(q, g g q)$ antenna function reads at leading colour:

$$
\mathcal{A}_{q, g g q}^{0}=
$$




$$
\begin{aligned}
& +\frac{1}{\epsilon^{4}}[3 \delta(1-z)] \\
& +\frac{1}{\epsilon^{3}}\left[3+\frac{19}{3} \delta(1-z)-6 \mathcal{D}_{0}(z)+3 z\right] \\
& +\frac{1}{\epsilon^{2}}\left[\frac{40}{3}+\delta(1-z)\left(\frac{1429}{72}-\frac{7 \pi^{2}}{3}\right)-\frac{38}{3} \mathcal{D}_{0}(z)+12 \mathcal{D}_{1}(z)+\frac{13}{2} \mathrm{H}(0 ; z)+6 \mathrm{H}(1 ; z)-\frac{12}{1-z} \mathrm{H}(0 ; z)\right. \\
& \left.+z\left(\frac{4}{3}+\frac{13}{2} \mathrm{H}(0 ; z)+6 \mathrm{H}(1 ; z)\right)\right] \\
& +\frac{1}{\epsilon}\left[\frac{865}{18}-\frac{7 \pi^{2}}{3}+\delta(1-z)\left(\frac{23959}{432}-\frac{181 \pi^{2}}{36}-25 \zeta_{3}\right)+\left(-\frac{1429}{36}+\frac{14 \pi^{2}}{3}\right) \mathcal{D}_{0}(z)+\frac{76}{3} \mathcal{D}_{1}(z)-12 \mathcal{D}_{2}(z)\right. \\
& +\frac{61}{2} \mathrm{H}(0 ; z)+\frac{80}{3} \mathrm{H}(1 ; z)+\frac{29}{2} \mathrm{H}(0,0 ; z)+13 \mathrm{H}(0,1 ; z)+13 \mathrm{H}(1,0 ; z)+12 \mathrm{H}(1,1 ; z) \\
& +\frac{1}{1-z}\left(-\frac{\pi^{2}}{3}-\frac{55}{2} \mathrm{H}(0 ; z)-26 \mathrm{H}(0,0 ; z)-24 \mathrm{H}(0,1 ; z)-26 \mathrm{H}(1,0 ; z)\right)+z\left(\frac{38}{9}-\frac{7 \pi^{2}}{3}\right. \\
& \left.\left.+\frac{9}{2} \mathrm{H}(0 ; z)+\frac{8}{3} \mathrm{H}(1 ; z)+\frac{29}{2} \mathrm{H}(0,0 ; z)+13 \mathrm{H}(0,1 ; z)+13 \mathrm{H}(1,0 ; z)+12 \mathrm{H}(1,1 ; z)\right)\right] \\
& +\left[\frac{3997}{27}-\frac{217 \pi^{2}}{18}-31 \zeta_{3}+\delta(1-z)\left(\frac{389623}{2592}-\frac{6857 \pi^{2}}{432}+\frac{163 \pi^{4}}{360}-\frac{1027 \zeta_{3}}{18}\right)+\mathcal{D}_{0}(z)\left(-\frac{23959}{216}\right.\right. \\
& \left.+\frac{181 \pi^{2}}{18}+50 \zeta_{3}\right)+\left(\frac{1429}{18}-\frac{28 \pi^{2}}{3}\right) \mathcal{D}_{1}(z)-\frac{76}{3} \mathcal{D}_{2}(z)+8 \mathcal{D}_{3}(z)+\frac{653}{6} \mathrm{H}(0 ; z)-\frac{71}{12} \pi^{2} \mathrm{H}(0 ; z) \\
& +\frac{865}{9} \mathrm{H}(1 ; z)-\frac{14}{3} \pi^{2} \mathrm{H}(1 ; z)+\frac{395}{6} \mathrm{H}(0,0 ; z)+61 \mathrm{H}(0,1 ; z)+\frac{163}{3} \mathrm{H}(1,0 ; z) \\
& +\frac{160}{3} \mathrm{H}(1,1 ; z)+\frac{61}{2} \mathrm{H}(0,0,0 ; z)+29 \mathrm{H}(0,0,1 ; z)+26 \mathrm{H}(0,1,0 ; z)+26 \mathrm{H}(0,1,1 ; z) \\
& +29 \mathrm{H}(1,0,0 ; z)+26 \mathrm{H}(1,0,1 ; z)+26 \mathrm{H}(1,1,0 ; z)+24 \mathrm{H}(1,1,1 ; z)+z\left(\frac{220}{27}-\frac{25 \pi^{2}}{18}\right. \\
& -31 \zeta_{3}+\frac{29}{3} \mathrm{H}(0 ; z)-\frac{71}{12} \pi^{2} \mathrm{H}(0 ; z)+\frac{76}{9} \mathrm{H}(1 ; z)-\frac{14}{3} \pi^{2} \mathrm{H}(1 ; z)+\frac{71}{6} \mathrm{H}(0,0 ; z) \\
& +9 \mathrm{H}(0,1 ; z)+\frac{19}{3} \mathrm{H}(1,0 ; z)+\frac{16}{3} \mathrm{H}(1,1 ; z)+\frac{61}{2} \mathrm{H}(0,0,0 ; z)+29 \mathrm{H}(0,0,1 ; z) \\
& +26 \mathrm{H}(0,1,0 ; z)+26 \mathrm{H}(0,1,1 ; z)+29 \mathrm{H}(1,0,0 ; z)+26 \mathrm{H}(1,0,1 ; z)+26 \mathrm{H}(1,1,0 ; z) \\
& +24 \mathrm{H}(1,1,1 ; z))+\frac{1}{1-z}\left(\frac{2 \pi^{2}}{9}+10 \zeta_{3}-\frac{274}{3} \mathrm{H}(0 ; z)+\frac{32}{3} \pi^{2} \mathrm{H}(0 ; z)-\frac{361}{6} \mathrm{H}(0,0 ; z)\right. \\
& -55 \mathrm{H}(0,1 ; z)-\frac{161}{3} \mathrm{H}(1,0 ; z)-54 \mathrm{H}(0,0,0 ; z)-52 \mathrm{H}(0,0,1 ; z)-48 \mathrm{H}(0,1,0 ; z) \\
& -48 \mathrm{H}(0,1,1 ; z)-58 \mathrm{H}(1,0,0 ; z)-52 \mathrm{H}(1,0,1 ; z)-52 \mathrm{H}(1,1,0 ; z))] \\
& +\mathcal{O}(\epsilon)
\end{aligned}
$$

and at subleading colour

$$
\begin{aligned}
& \tilde{\mathcal{A}}_{q, g g q}^{0}= \\
+ & \frac{1}{\epsilon^{4}}[4 \delta(1-z)] \\
+ & \frac{1}{\epsilon^{3}}\left[4+6 \delta(1-z)-8 \mathcal{D}_{0}(z)+4 z\right] \\
+ & \frac{1}{\epsilon^{2}}\left[13+\delta(1-z)\left(\frac{75}{4}-\frac{10 \pi^{2}}{3}\right)-12 \mathcal{D}_{0}(z)+16 \mathcal{D}_{1}(z)+9 \mathrm{H}(0 ; z)+8 \mathrm{H}(1 ; z)-\frac{16}{1-z} \mathrm{H}(0 ; z)\right.
\end{aligned}
$$




$$
\begin{aligned}
& +z(1+9 \mathrm{H}(0 ; z)+8 \mathrm{H}(1 ; z))] \\
& +\frac{1}{\epsilon}\left[48-\frac{11 \pi^{2}}{3}+\delta(1-z)\left(\frac{417}{8}-5 \pi^{2}-\frac{116 \zeta_{3}}{3}\right)+\left(-\frac{75}{2}+\frac{20 \pi^{2}}{3}\right) \mathcal{D}_{0}(z)+24 \mathcal{D}_{1}(z)-16 \mathcal{D}_{2}(z)\right. \\
& +30 \mathrm{H}(0 ; z)+26 \mathrm{H}(1 ; z)+21 \mathrm{H}(0,0 ; z)+18 \mathrm{H}(0,1 ; z)+16 \mathrm{H}(1,0 ; z)+16 \mathrm{H}(1,1 ; z) \\
& +\frac{1}{1-z}(-24 \mathrm{H}(0 ; z)-36 \mathrm{H}(0,0 ; z)-32 \mathrm{H}(0,1 ; z)-32 \mathrm{H}(1,0 ; z))+z\left(4-\frac{11 \pi^{2}}{3}\right. \\
& +2 \mathrm{H}(0 ; z)+2 \mathrm{H}(1 ; z)+21 \mathrm{H}(0,0 ; z)+18 \mathrm{H}(0,1 ; z)+16 \mathrm{H}(1,0 ; z)+16 \mathrm{H}(1,1 ; z))] \\
& +\left[157-\frac{77 \pi^{2}}{6}-\frac{170 \zeta_{3}}{3}+\delta(1-z)\left(\frac{2275}{16}-\frac{125 \pi^{2}}{8}+\frac{61 \pi^{4}}{90}-64 \zeta_{3}\right)+\mathcal{D}_{0}(z)\left(-\frac{417}{4}+10 \pi^{2}\right.\right. \\
& \left.+\frac{232 \zeta_{3}}{3}\right)+\left(75-\frac{40 \pi^{2}}{3}\right) \mathcal{D}_{1}(z)-24 \mathcal{D}_{2}(z)+\frac{32}{3} \mathcal{D}_{3}(z)+103 \mathrm{H}(0 ; z)-\frac{59}{6} \pi^{2} \mathrm{H}(0 ; z)+96 \mathrm{H}(1 ; z) \\
& -\frac{20}{3} \pi^{2} \mathrm{H}(1 ; z)+72 \mathrm{H}(0,0 ; z)+60 \mathrm{H}(0,1 ; z)+50 \mathrm{H}(1,0 ; z)+52 \mathrm{H}(1,1 ; z) \\
& +45 \mathrm{H}(0,0,0 ; z)+42 \mathrm{H}(0,0,1 ; z)+32 \mathrm{H}(0,1,0 ; z)+36 \mathrm{H}(0,1,1 ; z)+32 \mathrm{H}(1,0,0 ; z) \\
& +32 \mathrm{H}(1,0,1 ; z)+32 \mathrm{H}(1,1,0 ; z)+32 \mathrm{H}(1,1,1 ; z)+\frac{1}{1-z}\left(+24 \zeta_{3}-80 \mathrm{H}(0 ; z)\right. \\
& +\frac{52}{3} \pi^{2} \mathrm{H}(0 ; z)-60 \mathrm{H}(0,0 ; z)-48 \mathrm{H}(0,1 ; z)-48 \mathrm{H}(1,0 ; z)-76 \mathrm{H}(0,0,0 ; z) \\
& -72 \mathrm{H}(0,0,1 ; z)-56 \mathrm{H}(0,1,0 ; z)-64 \mathrm{H}(0,1,1 ; z)-64 \mathrm{H}(1,0,0 ; z)-64 \mathrm{H}(1,0,1 ; z) \\
& -64 \mathrm{H}(1,1,0 ; z))+z\left(7+\frac{7 \pi^{2}}{6}-\frac{170 \zeta_{3}}{3}+5 \mathrm{H}(0 ; z)-\frac{59}{6} \pi^{2} \mathrm{H}(0 ; z)+8 \mathrm{H}(1 ; z)\right. \\
& -\frac{20}{3} \pi^{2} \mathrm{H}(1 ; z)+8 \mathrm{H}(0,0 ; z)+4 \mathrm{H}(0,1 ; z)+10 \mathrm{H}(1,0 ; z)+4 \mathrm{H}(1,1 ; z)+45 \mathrm{H}(0,0,0 ; z) \\
& +42 \mathrm{H}(0,0,1 ; z)+32 \mathrm{H}(0,1,0 ; z)+36 \mathrm{H}(0,1,1 ; z)+32 \mathrm{H}(1,0,0 ; z)+32 \mathrm{H}(1,0,1 ; z) \\
& +32 \mathrm{H}(1,1,0 ; z)+32 \mathrm{H}(1,1,1 ; z))] \\
& +\mathcal{O}(\epsilon)
\end{aligned}
$$

Crossing a primary quark (coupled to the external current) into the initial state, one obtains the $\left(q, q^{\prime} \bar{q}^{\prime} q\right)$ antenna function, whose integral yields

$$
\begin{aligned}
& \mathcal{B}_{q, q^{\prime} \bar{q}^{\prime} q}^{0}= \\
+ & \frac{1}{\epsilon^{3}}\left[-\frac{1}{3} \delta(1-z)\right] \\
+ & \frac{1}{\epsilon^{2}}\left[-\frac{1}{3}-\frac{19}{18} \delta(1-z)+\frac{2}{3} \mathcal{D}_{0}(z)-\frac{z}{3}\right] \\
+ & \frac{1}{\epsilon}\left[-\frac{17}{9}+\delta(1-z)\left(-\frac{373}{108}+\frac{5 \pi^{2}}{18}\right)+\frac{19}{9} \mathcal{D}_{0}(z)-\frac{4}{3} \mathcal{D}_{1}(z)-\mathrm{H}(0 ; z)-\frac{2}{3} \mathrm{H}(1 ; z)+\frac{2}{1-z} \mathrm{H}(0 ; z)\right. \\
& +\quad\left[-\frac{241}{27}+\frac{7 \pi^{2}}{18}+\delta(1-z)\left(-\frac{7081}{648}+\frac{95 \pi^{2}}{108}+\frac{32 \zeta_{3}}{9}\right)+\left(\frac{373}{54}-\frac{5 \pi^{2}}{9}\right) \mathcal{D}_{0}(z)-\frac{38}{9} \mathcal{D}_{1}(z)+\frac{4}{3} \mathcal{D}_{2}(z)\right. \\
& \quad-\frac{19}{3} \mathrm{H}(0 ; z)-\frac{34}{9} \mathrm{H}(1 ; z)-\frac{7}{3} \mathrm{H}(0,0 ; z)-2 \mathrm{H}(0,1 ; z)-\frac{4}{3} \mathrm{H}(1,0 ; z)-\frac{4}{3} \mathrm{H}(1,1 ; z)
\end{aligned}
$$




$$
\begin{aligned}
& +\frac{1}{1-z}\left(-\frac{2 \pi^{2}}{9}+\frac{22}{3} \mathrm{H}(0 ; z)+\frac{14}{3} \mathrm{H}(0,0 ; z)+4 \mathrm{H}(0,1 ; z)+\frac{8}{3} \mathrm{H}(1,0 ; z)\right)+z\left(-\frac{19}{27}\right. \\
& \left.\left.+\frac{7 \pi^{2}}{18}-\frac{5}{3} \mathrm{H}(0 ; z)-\frac{10}{9} \mathrm{H}(1 ; z)-\frac{7}{3} \mathrm{H}(0,0 ; z)-2 \mathrm{H}(0,1 ; z)-\frac{4}{3} \mathrm{H}(1,0 ; z)-\frac{4}{3} \mathrm{H}(1,1 ; z)\right)\right] \\
& +\mathcal{O}(\epsilon) .
\end{aligned}
$$

Correspondingly, crossing a secondary quark (not coupled to the external current) into the initial state, one obtains the $\left(q^{\prime}, q \bar{q} q^{\prime}\right)$ antenna function, whose integral yields

$$
\begin{aligned}
& \mathcal{B}_{q^{\prime}, q \bar{q} q^{\prime}}^{0} \\
& +\frac{1}{\epsilon^{2}}\left[1+2 \mathrm{H}(0 ; z)+\frac{4}{3 z}-\frac{4}{3} z^{2}+z(-1+2 \mathrm{H}(0 ; z))\right] \\
& +\frac{1}{\epsilon}\left[\frac{10}{3}-\frac{2 \pi^{2}}{3}+\mathrm{H}(0 ; z)+2 \mathrm{H}(1 ; z)+6 \mathrm{H}(0,0 ; z)+4 \mathrm{H}(0,1 ; z)+\frac{1}{z}\left(\frac{4}{9}+\frac{8}{3} \mathrm{H}(1 ; z)\right)\right. \\
& \quad+z\left(-\frac{22}{3}-\frac{2 \pi^{2}}{3}-7 \mathrm{H}(0 ; z)-2 \mathrm{H}(1 ; z)+6 \mathrm{H}(0,0 ; z)+4 \mathrm{H}(0,1 ; z)\right) \\
& \left.\quad+z^{2}\left(\frac{32}{9}-\frac{16}{3} \mathrm{H}(0 ; z)-\frac{8}{3} \mathrm{H}(1 ; z)\right)\right] \\
& +\left[\frac{25}{9}-\frac{\pi^{2}}{2}-4 \zeta_{3}+\frac{88}{3} \mathrm{H}(0 ; z)-\frac{7}{3} \pi^{2} \mathrm{H}(0 ; z)+\frac{20}{3} \mathrm{H}(1 ; z)-8 \mathrm{H}(-1,0 ; z)+\mathrm{H}(0,0 ; z)\right. \\
& \quad+2 \mathrm{H}(0,1 ; z)+4 \mathrm{H}(1,0 ; z)+z^{2}\left(-\frac{208}{27}+2 \pi^{2}+\frac{128}{9} \mathrm{H}(0 ; z)+\frac{64}{9} \mathrm{H}(1 ; z)-\frac{8}{3} \mathrm{H}(-1,0 ; z)\right. \\
& \left.\quad-\frac{40}{3} \mathrm{H}(0,0 ; z)-\frac{32}{3} \mathrm{H}(0,1 ; z)-\frac{16}{3} \mathrm{H}(1,0 ; z)-\frac{16}{3} \mathrm{H}(1,1 ; z)\right)+4 \mathrm{H}(1,1 ; z)+14 \mathrm{H}(0,0,0 ; z) \\
& \quad+12 \mathrm{H}(0,0,1 ; z)+8 \mathrm{H}(0,1,0 ; z)+8 \mathrm{H}(0,1,1 ; z)+\frac{1}{z}\left(\frac{532}{27}-\frac{2 \pi^{2}}{3}+\frac{8}{9} \mathrm{H}(1 ; z)\right. \\
& \left.\quad-\frac{8}{3} \mathrm{H}(-1,0 ; z)+\frac{16}{3} \mathrm{H}(1,0 ; z)+\frac{16}{3} \mathrm{H}(1,1 ; z)\right)+z\left(-\frac{133}{9}+\frac{7 \pi^{2}}{6}-4 \zeta_{3}-4 \mathrm{H}(0 ; z)\right. \\
& \quad-\frac{7}{3} \pi^{2} \mathrm{H}(0 ; z)-\frac{44}{3} \mathrm{H}(1 ; z)-8 \mathrm{H}(-1,0 ; z)-11 \mathrm{H}(0,0 ; z)-14 \mathrm{H}(0,1 ; z)-4 \mathrm{H}(1,0 ; z) \\
& \quad-4 \mathrm{H}(1,1 ; z)+14 \mathrm{H}(0,0,0 ; z)+12 \mathrm{H}(0,0,1 ; z)+8 \mathrm{H}(0,1,0 ; z)+8 \mathrm{H}(0,1,1 ; z))] \\
& +\mathcal{O}(\epsilon) .
\end{aligned}
$$

As explained in section 2 above, there are three different crossings for the identical flavour only antenna functions $C_{4}^{0}$, which are symmetrized over the antiquarks, but not over the quarks.

Crossing either antiquark into the initial state yields the $(q, q \bar{q} q)$ antenna, whose integral is:

$$
\begin{aligned}
& \mathcal{C}_{q, q \bar{q} q}^{0}= \\
& +\frac{1}{\epsilon}\left[2-\frac{\pi^{2}}{12}+\frac{1}{2} \mathrm{H}(0 ; z)-\frac{1}{2} \mathrm{H}(0,0 ; z)-\frac{1}{2} \mathrm{H}(1,0 ; z)+\frac{1}{1-z}\left(\frac{\pi^{2}}{6}+\frac{3}{4} \mathrm{H}(0 ; z)+\mathrm{H}(0,0 ; z)\right.\right. \\
& \left.\quad+\mathrm{H}(1,0 ; z))+z\left(-\frac{7}{4}-\frac{\pi^{2}}{12}+\frac{1}{2} \mathrm{H}(0 ; z)-\frac{1}{2} \mathrm{H}(0,0 ; z)-\frac{1}{2} \mathrm{H}(1,0 ; z)\right)\right]
\end{aligned}
$$




$$
\begin{aligned}
+\left[\frac{1}{4}\right. & -\frac{5 \pi^{2}}{12}-\frac{3 \zeta_{3}}{2}-\frac{1}{6} \pi^{2} \mathrm{H}(0 ; z)+4 \mathrm{H}(1 ; z)+2 \mathrm{H}(-1,0 ; z)-2 \mathrm{H}(0,0 ; z)+\mathrm{H}(0,1 ; z) \\
& -\frac{5}{2} \mathrm{H}(1,0 ; z)-2 \mathrm{H}(0,-1,0 ; z)-\frac{3}{2} \mathrm{H}(0,0,0 ; z)-\mathrm{H}(0,0,1 ; z)-\mathrm{H}(0,1,0 ; z) \\
& -\frac{7}{2} \mathrm{H}(1,0,0 ; z)-\mathrm{H}(1,0,1 ; z)-\mathrm{H}(1,1,0 ; z)+\frac{1}{1-z}\left(\frac{\pi^{2}}{4}+3 \zeta_{3}+3 \mathrm{H}(0 ; z)+\frac{1}{3} \pi^{2} \mathrm{H}(0 ; z)\right. \\
& +\frac{21}{4} \mathrm{H}(0,0 ; z)+\frac{3}{2} \mathrm{H}(0,1 ; z)+3 \mathrm{H}(1,0 ; z)+4 \mathrm{H}(0,-1,0 ; z)+3 \mathrm{H}(0,0,0 ; z) \\
& +2 \mathrm{H}(0,0,1 ; z)+2 \mathrm{H}(0,1,0 ; z)+7 \mathrm{H}(1,0,0 ; z)+2 \mathrm{H}(1,0,1 ; z)+2 \mathrm{H}(1,1,0 ; z)) \\
& +z\left(\frac{7}{4}+\frac{\pi^{2}}{12}-\frac{27}{4} \mathrm{H}(0 ; z)-\frac{1}{6} \pi^{2} \mathrm{H}(0 ; z)-\frac{7}{2} \mathrm{H}(1 ; z)+2 \mathrm{H}(-1,0 ; z)+\mathrm{H}(0,0 ; z)\right. \\
& +\mathrm{H}(0,1 ; z)+\frac{1}{2} \mathrm{H}(1,0 ; z)-2 \mathrm{H}(0,-1,0 ; z)-\frac{3}{2} \mathrm{H}(0,0,0 ; z)-\mathrm{H}(0,0,1 ; z)-\mathrm{H}(0,1,0 ; z) \\
& \left.\left.-\frac{7}{2} \mathrm{H}(1,0,0 ; z)-\mathrm{H}(1,0,1 ; z)-\mathrm{H}(1,1,0 ; z)-\frac{3 \zeta_{3}}{2}\right)\right] \\
+ & \mathcal{O}(\epsilon) .
\end{aligned}
$$

Crossing the quark which couples to the external current to the initial state yields the $(\bar{q}, \bar{q} q \bar{q})$ antenna function, which integrates to:

$$
\begin{aligned}
& \mathcal{C}_{\bar{q}, \bar{q} q \bar{q}}^{0}= \\
& +\frac{1}{\epsilon}\left[\delta(1-z)\left(-\frac{13}{8}+\frac{\pi^{2}}{4}-\zeta_{3}\right)\right] \\
& +\left[-5+2 \pi^{2}+\delta(1-z)\left(-\frac{175}{16}+\frac{\pi^{2}}{2}+\frac{17 \pi^{4}}{180}-\frac{107 \zeta_{3}}{2}\right)+\mathcal{D}_{0}(z)\left(\frac{13}{4}-\frac{\pi^{2}}{2}+2 \zeta_{3}\right)-5 \mathrm{H}(0 ; z)\right. \\
& \quad-6 \mathrm{H}(-1,0 ; z)+15 \mathrm{H}(0,0 ; z)+9 \mathrm{H}(1,0 ; z)-\frac{2}{z^{2}} \mathrm{H}(-1,0 ; z)+\frac{1}{z}(-2+2 \mathrm{H}(0 ; z) \\
& \quad-6 \mathrm{H}(-1,0 ; z))+z\left(2 \pi^{2}-2 \mathrm{H}(-1,0 ; z)+9 \mathrm{H}(0,0 ; z)+7 \mathrm{H}(1,0 ; z)\right)+\frac{1}{1-z}\left(-\frac{3 \pi^{2}}{2}\right. \\
& \quad-6 \zeta_{3}+10 \mathrm{H}(0 ; z)+\frac{2}{3} \pi^{2} \mathrm{H}(1 ; z)-15 \mathrm{H}(0,0 ; z)-9 \mathrm{H}(1,0 ; z)+4 \mathrm{H}(0,-1,0 ; z) \\
& \quad+8 \mathrm{H}(1,0,0 ; z)+4 \mathrm{H}(1,1,0 ; z))] \\
& +\mathcal{O}(\epsilon) .
\end{aligned}
$$

Finally, crossing the quark not coupled to the external current (and thus participating in the collinear splitting), one obtains the $(\bar{q}, q \bar{q} \bar{q})$ antenna function, integrating to:

$$
\begin{aligned}
& \mathcal{C}_{\bar{q}, q \bar{q} \bar{q}}^{0}= \\
& +\frac{1}{\epsilon}\left[-2-\frac{\pi^{2}}{6}-\mathrm{H}(0 ; z)-2 \mathrm{H}(-1,0 ; z)+\mathrm{H}(0,0 ; z)+\frac{1}{1+z}\left(\frac{\pi^{2}}{3}+4 \mathrm{H}(-1,0 ; z)-2 \mathrm{H}(0,0 ; z)\right)\right. \\
& \left.\quad+z\left(2+\frac{\pi^{2}}{6}-\mathrm{H}(0 ; z)+2 \mathrm{H}(-1,0 ; z)-\mathrm{H}(0,0 ; z)\right)\right]
\end{aligned}
$$




$$
\begin{aligned}
& +\left[\frac{15}{2}+\frac{\pi^{2}}{6}-5 \zeta_{3}+\pi^{2} \mathrm{H}(-1 ; z)-\frac{1}{2} \mathrm{H}(0 ; z)-\frac{1}{3} \pi^{2} \mathrm{H}(0 ; z)-4 \mathrm{H}(1 ; z)-4 \mathrm{H}(-1,0 ; z)\right. \\
& \quad-2 \mathrm{H}(0,0 ; z)-2 \mathrm{H}(0,1 ; z)+4 \mathrm{H}(-1,-1,0 ; z)-8 \mathrm{H}(-1,0,0 ; z)-4 \mathrm{H}(-1,0,1 ; z) \\
& \quad-4 \mathrm{H}(0,-1,0 ; z)+3 \mathrm{H}(0,0,0 ; z)+2 \mathrm{H}(0,0,1 ; z)-\frac{2}{z^{2}} \mathrm{H}(-1,0 ; z)+\frac{1}{z}(-2+2 \mathrm{H}(0 ; z) \\
& \quad+6 \mathrm{H}(-1,0 ; z))+\frac{1}{1+z}\left(+14 \zeta_{3}-\frac{8}{3} \pi^{2} \mathrm{H}(-1 ; z)-4 \mathrm{H}(0 ; z)+\frac{2}{3} \pi^{2} \mathrm{H}(0 ; z)\right. \\
& \quad-16 \mathrm{H}(-1,-1,0 ; z)+20 \mathrm{H}(-1,0,0 ; z)+8 \mathrm{H}(-1,0,1 ; z)+12 \mathrm{H}(0,-1,0 ; z) \\
& \quad-6 \mathrm{H}(0,0,0 ; z)-4 \mathrm{H}(0,0,1 ; z))+z\left(-\frac{11}{2}+\frac{\pi^{2}}{6}+5 \zeta_{3}-\pi^{2} \mathrm{H}(-1 ; z)+\frac{19}{2} \mathrm{H}(0 ; z)\right. \\
& \quad+\frac{1}{3} \pi^{2} \mathrm{H}(0 ; z)+4 \mathrm{H}(1 ; z)-4 \mathrm{H}(-1,0 ; z)-2 \mathrm{H}(0,0 ; z)-2 \mathrm{H}(0,1 ; z)-4 \mathrm{H}(-1,-1,0 ; z) \\
& \quad+8 \mathrm{H}(-1,0,0 ; z)+4 \mathrm{H}(-1,0,1 ; z)+4 \mathrm{H}(0,-1,0 ; z)-3 \mathrm{H}(0,0,0 ; z)-2 \mathrm{H}(0,0,1 ; z))] \\
& +\mathcal{O}(\epsilon) .
\end{aligned}
$$

The integrated one-loop quark-antiquark antenna functions at leading and subleading colour read:

$$
\begin{aligned}
\mathcal{A}_{q, g q}^{1}= \\
+\frac{1}{\epsilon^{4}}\left[-\frac{1}{4} \delta(1-z)\right] \\
+\frac{1}{\epsilon^{3}}\left[-\frac{1}{4}-\frac{53}{24} \delta(1-z)-\frac{z}{4}+\frac{1}{2} \mathcal{D}_{0}(z)\right] \\
+\frac{1}{\epsilon^{2}}\left[-\frac{13}{6}+\delta(1-z)\left(-\frac{43}{16}+\frac{5 \pi^{2}}{24}\right)+\frac{31}{12} \mathcal{D}_{0}(z)-\mathcal{D}_{1}(z)-\frac{3}{4} \mathrm{H}(0 ; z)-\frac{1}{2} \mathrm{H}(1 ; z)+\frac{3 \mathrm{H}(0 ; z)}{2(1-z)}\right. \\
\left.\quad-z\left(\frac{2}{3}+\frac{3}{4} \mathrm{H}(0 ; z)+\frac{1}{2} \mathrm{H}(1 ; z)\right)\right] \\
+\frac{1}{\epsilon}\left[-\frac{27}{4}+\frac{\pi^{2}}{6}+\delta(1-z)\left(-\frac{167}{24}+\frac{37 \pi^{2}}{48}+\frac{7 \zeta_{3}}{6}\right)+\left(4-\frac{5 \pi^{2}}{12}\right) \mathcal{D}_{0}(z)-\frac{10}{3} \mathcal{D}_{1}(z)+\mathcal{D}_{2}(z)-\frac{14}{3} \mathrm{H}(0 ; z)\right. \\
\quad-\frac{41}{12} \mathrm{H}(1 ; z)-2 \mathrm{H}(0,0 ; z)-\frac{5}{4} \mathrm{H}(0,1 ; z)-\frac{3}{2} \mathrm{H}(1,0 ; z)-\mathrm{H}(1,1 ; z)+\frac{1}{1-z}\left(\frac{\pi^{2}}{12}\right. \\
\left.\quad+\frac{49}{12} \mathrm{H}(0 ; z)+4 \mathrm{H}(0,0 ; z)+\frac{5}{2} \mathrm{H}(0,1 ; z)+3 \mathrm{H}(1,0 ; z)\right)+z\left(\frac{25}{24}+\frac{\pi^{2}}{6}-\frac{1}{6} \mathrm{H}(0 ; z)\right. \\
\left.\left.\quad-\frac{5}{12} \mathrm{H}(1 ; z)-2 \mathrm{H}(0,0 ; z)-\frac{5}{4} \mathrm{H}(0,1 ; z)-\frac{3}{2} \mathrm{H}(1,0 ; z)-\mathrm{H}(1,1 ; z)\right)\right] \\
+\left[-\frac{67}{4}+\frac{67 \pi^{2}}{48}+\frac{29 \zeta_{3}}{12}+\delta(1-z)\left(-\frac{773}{48}+\frac{23 \pi^{2}}{16}-\frac{67 \pi^{4}}{1440}+\frac{217 \zeta_{3}}{36}\right)+\left(\frac{257}{24}-\frac{13 \pi^{2}}{12}\right.\right. \\
\left.\quad-\frac{7 \zeta_{3}}{3}\right) \mathcal{D}_{0}(z)+\left(-\frac{53}{8}+\frac{5 \pi^{2}}{6}\right) \mathcal{D}_{1}(z)+\frac{29}{12} \mathcal{D}_{2}(z)-\frac{2}{3} \mathcal{D}_{3}(z)-\frac{117}{8} \mathrm{H}(0 ; z)+\frac{5}{8} \pi^{2} \mathrm{H}(0 ; z)-\frac{89}{8} \mathrm{H}(1 ; z) \\
\quad+\frac{1}{3} \pi^{2} \mathrm{H}(1 ; z)-\frac{119}{12} \mathrm{H}(0,0 ; z)-\frac{43}{6} \mathrm{H}(0,1 ; z)-\frac{89}{12} \mathrm{H}(1,0 ; z)-\frac{71}{12} \mathrm{H}(1,1 ; z) \\
\quad-\frac{9}{2} \mathrm{H}(0,0,0 ; z)-3 \mathrm{H}(0,0,1 ; z)-3 \mathrm{H}(0,1,0 ; z)-\frac{9}{4} \mathrm{H}(0,1,1 ; z)-4 \mathrm{H}(1,0,0 ; z) \\
\quad-\frac{5}{2} \mathrm{H}(1,0,1 ; z)-3 \mathrm{H}(1,1,0 ; z)+\frac{1}{1-z}\left(\frac{\pi^{2}}{8}-\frac{5 \zeta_{3}}{2}+\frac{69}{8} \mathrm{H}(0 ; z)-\frac{5}{4} \pi^{2} \mathrm{H}(0 ; z)\right.
\end{aligned}
$$




$$
\begin{aligned}
& +\frac{1}{6} \pi^{2} \mathrm{H}(1 ; z)+\frac{47}{6} \mathrm{H}(0,0 ; z)+\frac{67}{12} \mathrm{H}(0,1 ; z)+\frac{19}{3} \mathrm{H}(1,0 ; z)+9 \mathrm{H}(0,0,0 ; z) \\
& +6 \mathrm{H}(0,0,1 ; z)+6 \mathrm{H}(0,1,0 ; z)+\frac{9}{2} \mathrm{H}(0,1,1 ; z)+8 \mathrm{H}(1,0,0 ; z)+5 \mathrm{H}(1,0,1 ; z) \\
& +6 \mathrm{H}(1,1,0 ; z))+z\left(\frac{1}{8}-\frac{5 \pi^{2}}{48}+\frac{29 \zeta_{3}}{12}+\frac{49}{24} \mathrm{H}(0 ; z)+\frac{5}{8} \pi^{2} \mathrm{H}(0 ; z)+\frac{23}{12} \mathrm{H}(1 ; z)\right. \\
& +\frac{1}{3} \pi^{2} \mathrm{H}(1 ; z)+\frac{7}{12} \mathrm{H}(0,0 ; z)+\frac{1}{3} \mathrm{H}(0,1 ; z)+\frac{1}{12} \mathrm{H}(1,0 ; z)+\frac{1}{12} \mathrm{H}(1,1 ; z) \\
& -\frac{9}{2} \mathrm{H}(0,0,0 ; z)-3 \mathrm{H}(0,0,1 ; z)-3 \mathrm{H}(0,1,0 ; z)-\frac{9}{4} \mathrm{H}(0,1,1 ; z)-4 \mathrm{H}(1,0,0 ; z) \\
& \left.\left.-\frac{5}{2} \mathrm{H}(1,0,1 ; z)-3 \mathrm{H}(1,1,0 ; z)-2 \mathrm{H}(1,1,1 ; z)\right)-2 \mathrm{H}(1,1,1 ; z)\right] \\
& +\mathcal{O}(\epsilon) \\
& +\frac{1}{\epsilon^{2}}\left[\delta(1-z)\left(-\frac{5}{16}+\frac{\pi^{2}}{12}\right)-\frac{1}{4} \mathrm{H}(0 ; z)+\frac{\mathrm{H}(0 ; z)}{2(1-z)}-\frac{1}{4} z \mathrm{H}(0 ; z)\right] \\
& +\frac{1}{\epsilon}\left[-1+\frac{\pi^{2}}{8}+\delta(1-z)\left(-\frac{5}{4}+\frac{5 \zeta_{3}}{2}\right)+\left(\frac{5}{8}-\frac{\pi^{2}}{6}\right) \mathcal{D}_{0}(z)-\frac{5}{4} \mathrm{H}(0 ; z)-\mathrm{H}(0,0 ; z)-\frac{1}{4} \mathrm{H}(0,1 ; z)\right. \\
& +\frac{1}{1-z}\left(-\frac{\pi^{2}}{12}+\frac{3}{4} \mathrm{H}(0 ; z)+2 \mathrm{H}(0,0 ; z)+\frac{1}{2} \mathrm{H}(0,1 ; z)\right)+z\left(-\frac{1}{8}+\frac{\pi^{2}}{8}+\frac{1}{4} \mathrm{H}(0 ; z)\right. \\
& \left.\left.-\mathrm{H}(0,0 ; z)-\frac{1}{4} \mathrm{H}(0,1 ; z)\right)\right] \\
& +\left[-6-\frac{\pi^{2}}{24}+\frac{9 \zeta_{3}}{4}+\delta(1-z)\left(-\frac{61}{16}+\frac{25 \pi^{2}}{96}-\frac{43 \pi^{4}}{1440}+16 \zeta_{3}\right)+\left(\frac{5}{2}-5 \zeta_{3}\right) \mathcal{D}_{0}(z)+\left(-\frac{5}{4}+\frac{\pi^{2}}{3}\right) \mathcal{D}_{1}(z)\right. \\
& -\frac{35}{8} \mathrm{H}(0 ; z)+\frac{13}{24} \pi^{2} \mathrm{H}(0 ; z)-\frac{7}{8} \mathrm{H}(1 ; z)+\frac{1}{6} \pi^{2} \mathrm{H}(1 ; z)-7 \mathrm{H}(0,0 ; z)-\frac{5}{4} \mathrm{H}(0,1 ; z) \\
& -2 \mathrm{H}(1,0 ; z)-\frac{5}{2} \mathrm{H}(0,0,0 ; z)-\mathrm{H}(0,0,1 ; z)-\frac{1}{4} \mathrm{H}(0,1,1 ; z)+\frac{1}{2} \mathrm{H}(1,0,0 ; z) \\
& +\frac{1}{2} \mathrm{H}(1,1,0 ; z)+\frac{1}{1-z}\left(\frac{\pi^{2}}{8}+\frac{3 \zeta_{3}}{2}+\frac{7}{4} \mathrm{H}(0 ; z)-\frac{13}{12} \pi^{2} \mathrm{H}(0 ; z)-\frac{1}{3} \pi^{2} \mathrm{H}(1 ; z)\right. \\
& +6 \mathrm{H}(0,0 ; z)+\frac{3}{4} \mathrm{H}(0,1 ; z)+\frac{3}{2} \mathrm{H}(1,0 ; z)+5 \mathrm{H}(0,0,0 ; z)+2 \mathrm{H}(0,0,1 ; z)+\frac{1}{2} \mathrm{H}(0,1,1 ; z) \\
& -2 \mathrm{H}(1,0,0 ; z)-2 \mathrm{H}(1,1,0 ; z))+z\left(-\frac{1}{8}-\frac{23 \pi^{2}}{24}+\frac{9 \zeta_{3}}{4}+\frac{1}{8} \mathrm{H}(0 ; z)+\frac{13}{24} \pi^{2} \mathrm{H}(0 ; z)\right. \\
& +\frac{1}{4} \mathrm{H}(1 ; z)+\frac{1}{6} \pi^{2} \mathrm{H}(1 ; z)-\frac{3}{2} \mathrm{H}(0,0 ; z)+\frac{1}{4} \mathrm{H}(0,1 ; z)-\frac{5}{2} \mathrm{H}(1,0 ; z)-\frac{5}{2} \mathrm{H}(0,0,0 ; z) \\
& \left.\left.-\mathrm{H}(0,0,1 ; z)-\frac{1}{4} \mathrm{H}(0,1,1 ; z)+\frac{1}{2} \mathrm{H}(1,0,0 ; z)+\frac{1}{2} \mathrm{H}(1,1,0 ; z)\right)\right] \\
& +\mathcal{O}(\epsilon)
\end{aligned}
$$

The quark loop contribution is:

$$
\begin{gathered}
\hat{\mathcal{A}}_{q, g q}^{1}= \\
+\frac{1}{\epsilon^{3}}\left[\frac{1}{3} \delta(1-z)\right]
\end{gathered}
$$




$$
\begin{aligned}
+\frac{1}{\epsilon^{2}} & {\left[\frac{1}{6}+\frac{1}{4} \delta(1-z)-\frac{1}{3} \mathcal{D}_{0}(z)+\frac{z}{6}\right] } \\
+\frac{1}{\epsilon}[ & \frac{1}{2}+\delta(1-z)\left(\frac{7}{12}-\frac{\pi^{2}}{12}\right)-\frac{1}{4} \mathcal{D}_{0}(z)+\frac{1}{3} \mathcal{D}_{1}(z)+\frac{1}{6} \mathrm{H}(0 ; z)+\frac{1}{6} \mathrm{H}(1 ; z)-\frac{1}{3(1-z)} \mathrm{H}(0 ; z) \\
& \left.+z\left(-\frac{1}{6}+\frac{1}{6} \mathrm{H}(0 ; z)+\frac{1}{6} \mathrm{H}(1 ; z)\right)\right] \\
+[1 & -\frac{\pi^{2}}{24}+\delta(1-z)\left(\frac{7}{6}-\frac{\pi^{2}}{16}-\frac{7 \zeta_{3}}{9}\right)+\left(-\frac{7}{12}+\frac{\pi^{2}}{12}\right) \mathcal{D}_{0}(z)+\frac{1}{4} \mathcal{D}_{1}(z)-\frac{1}{6} \mathcal{D}_{2}(z)+\frac{1}{2} \mathrm{H}(0 ; z) \\
& +\frac{1}{2} \mathrm{H}(1 ; z)+\frac{1}{6} \mathrm{H}(0,0 ; z)+\frac{1}{6} \mathrm{H}(0,1 ; z)+\frac{1}{6} \mathrm{H}(1,0 ; z)+\frac{1}{6} \mathrm{H}(1,1 ; z)+\frac{1}{1-z}\left(-\frac{1}{4} \mathrm{H}(0 ; z)\right. \\
& \left.-\frac{1}{3} \mathrm{H}(0,0 ; z)-\frac{1}{3} \mathrm{H}(0,1 ; z)-\frac{1}{3} \mathrm{H}(1,0 ; z)\right)+z\left(-\frac{\pi^{2}}{24}-\frac{1}{6} \mathrm{H}(0 ; z)-\frac{1}{6} \mathrm{H}(1 ; z)+\frac{1}{6} \mathrm{H}(0,0 ; z)\right. \\
& \left.\left.+\frac{1}{6} \mathrm{H}(0,1 ; z)+\frac{1}{6} \mathrm{H}(1,0 ; z)+\frac{1}{6} \mathrm{H}(1,1 ; z)\right)\right] \\
+ & \mathcal{O}(\epsilon) .
\end{aligned}
$$

\subsection{Quark-gluon antennae}

The integrated NLO quark-gluon initial-final antenna with an initial state quark reads through to $\epsilon^{2}$ for the gluon radiation:

$$
\begin{aligned}
& \mathcal{D}_{q, g g}^{0}= \\
& +\frac{1}{\epsilon^{2}}[2 \delta(1-z)] \\
& +\frac{1}{\epsilon}\left[1+\frac{11}{6} \delta(1-z)+z-2 \mathcal{D}_{0}(z)\right] \\
& +\left[1+\delta(1-z)\left(\frac{67}{18}-\frac{\pi^{2}}{2}\right)-\frac{1}{3 z}-\frac{11}{6} \mathcal{D}_{0}(z)+2 \mathcal{D}_{1}(z)+\mathrm{H}(0 ; z)+\mathrm{H}(1 ; z)-\frac{2}{1-z} \mathrm{H}(0 ; z)\right. \\
& \quad+z(-1+\mathrm{H}(0 ; z)+\mathrm{H}(1 ; z))] \\
& +\epsilon\left[1-\frac{\pi^{2}}{4}+\delta(1-z)\left(\frac{202}{27}-\frac{11 \pi^{2}}{24}-\frac{14 \zeta_{3}}{3}\right)+\left(-\frac{67}{18}+\frac{\pi^{2}}{2}\right) \mathcal{D}_{0}(z)+\frac{11}{6} \mathcal{D}_{1}(z)-\mathcal{D}_{2}(z)+\mathrm{H}(0 ; z)\right. \\
& \quad+\mathrm{H}(1 ; z)+\mathrm{H}(0,0 ; z)+\mathrm{H}(0,1 ; z)+\mathrm{H}(1,0 ; z)+\mathrm{H}(1,1 ; z)-\frac{1}{z}\left(\frac{2}{9}+\frac{1}{3} \mathrm{H}(0 ; z)+\frac{1}{3} \mathrm{H}(1 ; z)\right) \\
& \quad+\frac{1}{1-z}\left(-\frac{11}{6} \mathrm{H}(0 ; z)-2 \mathrm{H}(0,0 ; z)-2 \mathrm{H}(0,1 ; z)-2 \mathrm{H}(1,0 ; z)\right)+z\left(-\frac{\pi^{2}}{4}-\mathrm{H}(0 ; z)\right. \\
& \quad-\mathrm{H}(1 ; z)+\mathrm{H}(0,0 ; z)+\mathrm{H}(0,1 ; z)+\mathrm{H}(1,0 ; z)+\mathrm{H}(1,1 ; z))] \\
& +\epsilon^{2}\left[3-\frac{\pi^{2}}{4}-\frac{7 \zeta_{3}}{3}+\delta(1-z)\left(\frac{1214}{81}-\frac{67 \pi^{2}}{72}-\frac{\pi^{4}}{48}-\frac{77 \zeta_{3}}{18}\right)+\mathcal{D}_{0}(z)\left(-\frac{202}{27}+\frac{11 \pi^{2}}{24}+\frac{14 \zeta_{3}}{3}\right)\right. \\
& \quad+\left(\frac{67}{18}-\frac{\pi^{2}}{2}\right) \mathcal{D}_{1}(z)-\frac{11}{12} \mathcal{D}_{2}(z)+\frac{1}{3} \mathcal{D}_{3}(z)+\mathrm{H}(0 ; z)-\frac{1}{4} \pi^{2} \mathrm{H}(0 ; z)+\mathrm{H}(1 ; z)-\frac{1}{4} \pi^{2} \mathrm{H}(1 ; z) \\
& \quad+\mathrm{H}(0,0 ; z)+\mathrm{H}(0,1 ; z)+\mathrm{H}(1,0 ; z)+\mathrm{H}(1,1 ; z)+\mathrm{H}(0,0,0 ; z)+\mathrm{H}(0,0,1 ; z)
\end{aligned}
$$




$$
\begin{aligned}
& +\mathrm{H}(0,1,0 ; z)+\mathrm{H}(0,1,1 ; z)+\mathrm{H}(1,0,0 ; z)+\mathrm{H}(1,0,1 ; z)+\mathrm{H}(1,1,0 ; z)+\mathrm{H}(1,1,1 ; z) \\
& +\frac{1}{z}\left(-\frac{13}{27}+\frac{\pi^{2}}{12}-\frac{2}{9} \mathrm{H}(0 ; z)-\frac{2}{9} \mathrm{H}(1 ; z)-\frac{1}{3} \mathrm{H}(0,0 ; z)-\frac{1}{3} \mathrm{H}(0,1 ; z)-\frac{1}{3} \mathrm{H}(1,0 ; z)\right. \\
& \left.-\frac{1}{3} \mathrm{H}(1,1 ; z)\right)+\frac{1}{1-z}\left(-\frac{67}{18} \mathrm{H}(0 ; z)+\frac{1}{2} \pi^{2} \mathrm{H}(0 ; z)-\frac{11}{6} \mathrm{H}(0,0 ; z)-\frac{11}{6} \mathrm{H}(0,1 ; z)\right. \\
& -\frac{11}{6} \mathrm{H}(1,0 ; z)-2 \mathrm{H}(0,0,0 ; z)-2 \mathrm{H}(0,0,1 ; z)-2 \mathrm{H}(0,1,0 ; z)-2 \mathrm{H}(0,1,1 ; z) \\
& -2 \mathrm{H}(1,0,0 ; z)-2 \mathrm{H}(1,0,1 ; z)-2 \mathrm{H}(1,1,0 ; z))+z\left(\frac{\pi^{2}}{4}-\frac{7 \zeta_{3}}{3}-\frac{1}{4} \pi^{2} \mathrm{H}(0 ; z)\right. \\
& -\frac{1}{4} \pi^{2} \mathrm{H}(1 ; z)-\mathrm{H}(0,0 ; z)-\mathrm{H}(0,1 ; z)-\mathrm{H}(1,0 ; z)-\mathrm{H}(1,1 ; z)+\mathrm{H}(0,0,0 ; z) \\
& +\mathrm{H}(0,0,1 ; z)+\mathrm{H}(0,1,0 ; z)+\mathrm{H}(0,1,1 ; z)+\mathrm{H}(1,0,0 ; z)+\mathrm{H}(1,0,1 ; z)+\mathrm{H}(1,1,0 ; z) \\
& +\mathrm{H}(1,1,1 ; z))] \\
& +\mathcal{O}\left(\epsilon^{3}\right)
\end{aligned}
$$

while we have to distinguish two cases for the secondary quark radiation. Crossing the primary quark into the initial state, one obtains

$$
\begin{aligned}
& \mathcal{E}_{q, q^{\prime} \bar{q}^{\prime}}^{0}= \\
& -\frac{1}{\epsilon}\left[\frac{1}{3} \delta(1-z)\right] \\
& -\left[\frac{5}{9} \delta(1-z)+\frac{1}{6 z}-\frac{1}{3} \mathcal{D}_{0}(z)\right] \\
& +\epsilon\left[\delta(1-z)\left(-\frac{28}{27}+\frac{\pi^{2}}{12}\right)+\frac{5}{9} \mathcal{D}_{0}(z)-\frac{1}{3} \mathcal{D}_{1}(z)+\frac{1}{3(1-z)} \mathrm{H}(0 ; z)\right. \\
& \left.\quad-\frac{1}{z}\left(\frac{4}{9}+\frac{1}{6} \mathrm{H}(0 ; z)+\frac{1}{6} \mathrm{H}(1 ; z)\right)\right] \\
& +\epsilon^{2}\left[+\delta(1-z)\left(-\frac{164}{81}+\frac{5 \pi^{2}}{36}+\frac{7 \zeta_{3}}{9}\right)+\left(\frac{28}{27}-\frac{\pi^{2}}{12}\right) \mathcal{D}_{0}(z)-\frac{5}{9} \mathcal{D}_{1}(z)+\frac{1}{6} \mathcal{D}_{2}(z)+\frac{1}{1-z}\left(\frac{5}{9} \mathrm{H}(0 ; z)\right.\right. \\
& \left.\quad+\frac{1}{3} \mathrm{H}(0,0 ; z)+\frac{1}{3} \mathrm{H}(0,1 ; z)+\frac{1}{3} \mathrm{H}(1,0 ; z)\right)+\frac{1}{z}\left(-\frac{26}{27}+\frac{\pi^{2}}{24}-\frac{4}{9} \mathrm{H}(0 ; z)-\frac{4}{9} \mathrm{H}(1 ; z)\right. \\
& \left.\left.\quad-\frac{1}{6} \mathrm{H}(0,0 ; z)-\frac{1}{6} \mathrm{H}(0,1 ; z)-\frac{1}{6} \mathrm{H}(1,0 ; z)-\frac{1}{6} \mathrm{H}(1,1 ; z)\right)\right] \\
& \quad+\mathcal{O}\left(\epsilon^{3}\right),
\end{aligned}
$$

while crossing the secondary quark results in

$$
\begin{aligned}
& \mathcal{E}_{q^{\prime}, q^{\prime} q}^{0}= \\
+ & \frac{1}{\epsilon}\left[-1+\frac{1}{z}+\frac{z}{2}\right] \\
+ & {\left[-\frac{3}{2}-\mathrm{H}(0 ; z)-\mathrm{H}(1 ; z)+\frac{1}{z}(2+\mathrm{H}(0 ; z)+\mathrm{H}(1 ; z))+z\left(\frac{1}{2} \mathrm{H}(0 ; z)+\frac{1}{2} \mathrm{H}(1 ; z)\right)\right] }
\end{aligned}
$$




$$
\begin{aligned}
+\epsilon[ & -3+\frac{\pi^{2}}{4}-\frac{3}{2} \mathrm{H}(0 ; z)-\frac{3}{2} \mathrm{H}(1 ; z)-\mathrm{H}(0,0 ; z)-\mathrm{H}(0,1 ; z)-\mathrm{H}(1,0 ; z)-\mathrm{H}(1,1 ; z) \\
& +\frac{1}{z}\left(4-\frac{\pi^{2}}{4}+2 \mathrm{H}(0 ; z)+2 \mathrm{H}(1 ; z)+\mathrm{H}(0,0 ; z)+\mathrm{H}(0,1 ; z)+\mathrm{H}(1,0 ; z)+\mathrm{H}(1,1 ; z)\right) \\
& \left.+z\left(-\frac{\pi^{2}}{8}+\frac{1}{2} \mathrm{H}(0,0 ; z)+\frac{1}{2} \mathrm{H}(0,1 ; z)+\frac{1}{2} \mathrm{H}(1,0 ; z)+\frac{1}{2} \mathrm{H}(1,1 ; z)\right)\right] \\
+\epsilon^{2}[ & -6+\frac{3 \pi^{2}}{8}+\frac{7 \zeta_{3}}{3}-3 \mathrm{H}(0 ; z)+\frac{1}{4} \pi^{2} \mathrm{H}(0 ; z)-3 \mathrm{H}(1 ; z)+\frac{1}{4} \pi^{2} \mathrm{H}(1 ; z)-\frac{3}{2} \mathrm{H}(0,0 ; z) \\
& -\frac{3}{2} \mathrm{H}(0,1 ; z)-\frac{3}{2} \mathrm{H}(1,0 ; z)-\frac{3}{2} \mathrm{H}(1,1 ; z)-\mathrm{H}(0,0,0 ; z)-\mathrm{H}(0,0,1 ; z)-\mathrm{H}(0,1,0 ; z) \\
& -\mathrm{H}(0,1,1 ; z)-\mathrm{H}(1,0,0 ; z)-\mathrm{H}(1,0,1 ; z)-\mathrm{H}(1,1,0 ; z)-\mathrm{H}(1,1,1 ; z)+\frac{1}{z}\left(8-\frac{\pi^{2}}{2}\right. \\
& -\frac{7 \zeta_{3}}{3}+4 \mathrm{H}(0 ; z)-\frac{1}{4} \pi^{2} \mathrm{H}(0 ; z)+4 \mathrm{H}(1 ; z)-\frac{1}{4} \pi^{2} \mathrm{H}(1 ; z)+2 \mathrm{H}(0,0 ; z)+2 \mathrm{H}(0,1 ; z) \\
& +2 \mathrm{H}(1,0 ; z)+2 \mathrm{H}(1,1 ; z)+\mathrm{H}(0,0,0 ; z)+\mathrm{H}(0,0,1 ; z)+\mathrm{H}(0,1,0 ; z)+\mathrm{H}(0,1,1 ; z) \\
& +\mathrm{H}(1,0,0 ; z)+\mathrm{H}(1,0,1 ; z)+\mathrm{H}(1,1,0 ; z)+\mathrm{H}(1,1,1 ; z))+z\left(-\frac{7 \zeta_{3}}{6}-\frac{1}{8} \pi^{2} \mathrm{H}(0 ; z)\right. \\
& -\frac{1}{8} \pi^{2} \mathrm{H}(1 ; z)+\frac{1}{2} \mathrm{H}(0,0,0 ; z)+\frac{1}{2} \mathrm{H}(0,0,1 ; z)+\frac{1}{2} \mathrm{H}(0,1,0 ; z)+\frac{1}{2} \mathrm{H}(0,1,1 ; z) \\
& \left.\left.+\frac{1}{2} \mathrm{H}(1,0,0 ; z)+\frac{1}{2} \mathrm{H}(1,0,1 ; z)+\frac{1}{2} \mathrm{H}(1,1,0 ; z)+\frac{1}{2} \mathrm{H}(1,1,1 ; z)\right)\right] \\
+ & \mathcal{O}\left(\epsilon^{3}\right) .
\end{aligned}
$$

The NNLO double real radiation quark-gluon antenna functions are of the $D_{4}^{0}$ type (quark-gluon-gluon-gluon) and of $E_{4}^{0}$-type (quark-quark-antiquark-gluon). While the former has only one possible quark-initiated crossing, we have to distinguish several different cases for the latter.

The integrated form of the initial-final $(q, g g g)$ antenna function reads:

$$
\begin{aligned}
& \mathcal{D}_{q, g g g}^{0}= \\
& +\frac{1}{\epsilon^{4}}[10 \delta(1-z)] \\
& +\frac{1}{\epsilon^{3}}\left[10+22 \delta(1-z)-20 \mathcal{D}_{0}(z)+10 z\right] \\
& +\frac{1}{\epsilon^{2}}\left[17+\delta(1-z)\left(\frac{590}{9}-8 \pi^{2}\right)-44 \mathcal{D}_{0}(z)+40 \mathcal{D}_{1}(z)+22 \mathrm{H}(0 ; z)+20 \mathrm{H}(1 ; z)-\frac{40 \mathrm{H}(0 ; z)}{1-z}-\frac{4}{z}\right. \\
& \quad+z(5+22 \mathrm{H}(0 ; z)+20 \mathrm{H}(1 ; z))] \\
& +\frac{1}{\epsilon}\left[\frac{107}{3}-\frac{25 \pi^{2}}{3}+\delta(1-z)\left(\frac{4868}{27}-\frac{154 \pi^{2}}{9}-\frac{272 \zeta_{3}}{3}\right)+\left(-\frac{1180}{9}+16 \pi^{2}\right) \mathcal{D}_{0}(z)+88 \mathcal{D}_{1}(z)-40 \mathcal{D}_{2}(z)\right. \\
& \quad+\frac{113}{3} \mathrm{H}(0 ; z)+34 \mathrm{H}(1 ; z)+50 \mathrm{H}(0,0 ; z)+44 \mathrm{H}(0,1 ; z)+42 \mathrm{H}(1,0 ; z)+40 \mathrm{H}(1,1 ; z) \\
& \quad+\frac{1}{1-z}\left(-\frac{2 \pi^{2}}{3}-\frac{277}{3} \mathrm{H}(0 ; z)-88 \mathrm{H}(0,0 ; z)-80 \mathrm{H}(0,1 ; z)-84 \mathrm{H}(1,0 ; z)\right)+\frac{1}{z}\left(-\frac{112}{9}\right. \\
& \quad-8 \mathrm{H}(0 ; z)-8 \mathrm{H}(1 ; z))+z\left(22-\frac{25 \pi^{2}}{3}+\frac{41}{3} \mathrm{H}(0 ; z)+10 \mathrm{H}(1 ; z)+50 \mathrm{H}(0,0 ; z)\right.
\end{aligned}
$$




$$
\begin{aligned}
& +44 \mathrm{H}(0,1 ; z)+42 \mathrm{H}(1,0 ; z)+40 \mathrm{H}(1,1 ; z))] \\
& +\left[\frac{565}{9}-\frac{145 \pi^{2}}{18}-\frac{308 \zeta_{3}}{3}+\delta(1-z)\left(\frac{25811}{54}-\frac{1408 \pi^{2}}{27}+\frac{319 \pi^{4}}{180}-\frac{934 \zeta_{3}}{3}\right)+\mathcal{D}_{0}(z)\left(-\frac{9736}{27}\right.\right. \\
& \left.+\frac{308 \pi^{2}}{9}+\frac{544 \zeta_{3}}{3}\right)+\left(\frac{2360}{9}-32 \pi^{2}\right) \mathcal{D}_{1}(z)-88 \mathcal{D}_{2}(z)+\frac{80}{3} \mathcal{D}_{3}(z)-\frac{4}{3} \pi^{2} \mathrm{H}(-1 ; z)+\frac{616}{9} \mathrm{H}(0 ; z) \\
& -\frac{65}{3} \pi^{2} \mathrm{H}(0 ; z)+\frac{214}{3} \mathrm{H}(1 ; z)-\frac{50}{3} \pi^{2} \mathrm{H}(1 ; z)-8 \mathrm{H}(-1,0 ; z)+121 \mathrm{H}(0,0 ; z) \\
& +\frac{226}{3} \mathrm{H}(0,1 ; z)+96 \mathrm{H}(1,0 ; z)+68 \mathrm{H}(1,1 ; z)-16 \mathrm{H}(-1,-1,0 ; z)+8 \mathrm{H}(-1,0,0 ; z) \\
& +4 \mathrm{H}(0,-1,0 ; z)+106 \mathrm{H}(0,0,0 ; z)+100 \mathrm{H}(0,0,1 ; z)+84 \mathrm{H}(0,1,0 ; z)+88 \mathrm{H}(0,1,1 ; z) \\
& +82 \mathrm{H}(1,0,0 ; z)+84 \mathrm{H}(1,0,1 ; z)+80 \mathrm{H}(1,1,0 ; z)+80 \mathrm{H}(1,1,1 ; z)+\frac{1}{z}\left(-\frac{2087}{54}+\frac{34 \pi^{2}}{9}\right. \\
& -4 \zeta_{3}-\frac{2}{3} \pi^{2} \mathrm{H}(-1 ; z)-\frac{224}{9} \mathrm{H}(0 ; z)-\frac{224}{9} \mathrm{H}(1 ; z)+\frac{2}{3} \pi^{2} \mathrm{H}(1 ; z)-4 \mathrm{H}(-1,0 ; z) \\
& -16 \mathrm{H}(0,0 ; z)-16 \mathrm{H}(0,1 ; z)-\frac{44}{3} \mathrm{H}(1,0 ; z)-16 \mathrm{H}(1,1 ; z)-8 \mathrm{H}(-1,-1,0 ; z) \\
& +4 \mathrm{H}(-1,0,0 ; z)+8 \mathrm{H}(0,-1,0 ; z)+8 \mathrm{H}(1,0,0 ; z)+4 \mathrm{H}(1,1,0 ; z))+\frac{1}{1-z}\left(-\frac{11 \pi^{2}}{3}\right. \\
& +32 \zeta_{3}-\frac{2368}{9} \mathrm{H}(0 ; z)+\frac{116}{3} \pi^{2} \mathrm{H}(0 ; z)+\frac{4}{3} \pi^{2} \mathrm{H}(1 ; z)-243 \mathrm{H}(0,0 ; z)-\frac{554}{3} \mathrm{H}(0,1 ; z) \\
& -\frac{620}{3} \mathrm{H}(1,0 ; z)+8 \mathrm{H}(0,-1,0 ; z)-184 \mathrm{H}(0,0,0 ; z)-176 \mathrm{H}(0,0,1 ; z)-152 \mathrm{H}(0,1,0 ; z) \\
& -160 \mathrm{H}(0,1,1 ; z)-164 \mathrm{H}(1,0,0 ; z)-168 \mathrm{H}(1,0,1 ; z)-160 \mathrm{H}(1,1,0 ; z))+z\left(\frac{443}{6}\right. \\
& -\frac{25 \pi^{2}}{18}-\frac{320 \zeta_{3}}{3}-\frac{2}{3} \pi^{2} \mathrm{H}(-1 ; z)+\frac{391}{9} \mathrm{H}(0 ; z)-\frac{65}{3} \pi^{2} \mathrm{H}(0 ; z)+44 \mathrm{H}(1 ; z) \\
& -\frac{50}{3} \pi^{2} \mathrm{H}(1 ; z)-4 \mathrm{H}(-1,0 ; z)+47 \mathrm{H}(0,0 ; z)+\frac{82}{3} \mathrm{H}(0,1 ; z)+34 \mathrm{H}(1,0 ; z) \\
& +20 \mathrm{H}(1,1 ; z)-8 \mathrm{H}(-1,-1,0 ; z)+4 \mathrm{H}(-1,0,0 ; z)+106 \mathrm{H}(0,0,0 ; z)+100 \mathrm{H}(0,0,1 ; z) \\
& +84 \mathrm{H}(0,1,0 ; z)+88 \mathrm{H}(0,1,1 ; z)+82 \mathrm{H}(1,0,0 ; z)+84 \mathrm{H}(1,0,1 ; z)+80 \mathrm{H}(1,1,0 ; z) \\
& +80 \mathrm{H}(1,1,1 ; z))] \\
& +\mathcal{O}(\epsilon)
\end{aligned}
$$

For the $E_{4}^{0}$-type antenna functions, we can either cross the primary quark or the secondary quark into the initial state. In both cases, we have leading and subleading colour contributions.

Crossing the primary quark, one obtains the $\left(q, q^{\prime} \bar{q}^{\prime} g\right)$ antenna functions, whose integral reads at leading colour

$$
\begin{aligned}
& \mathcal{E}_{q, q^{\prime} \bar{q}^{\prime} g}^{0}= \\
+ & \frac{1}{\epsilon^{3}}\left[-\frac{5}{3} \delta(1-z)\right] \\
+ & \frac{1}{\epsilon^{2}}\left[-1-\frac{47}{9} \delta(1-z)+\frac{10}{3} \mathcal{D}_{0}(z)-\frac{2}{3 z}-z\right]
\end{aligned}
$$




$$
\begin{aligned}
+\frac{1}{\epsilon}[ & -\frac{4}{3}+\delta(1-z)\left(-\frac{557}{36}+\frac{23 \pi^{2}}{18}\right)+\frac{94}{9} \mathcal{D}_{0}(z)-\frac{20}{3} \mathcal{D}_{1}(z)-\frac{7}{3} \mathrm{H}(0 ; z)-2 \mathrm{H}(1 ; z)+\frac{22 \mathrm{H}(0 ; z)}{3(1-z)} \\
& \left.+\frac{1}{z}\left(-\frac{26}{9}-\frac{4}{3} \mathrm{H}(0 ; z)-\frac{4}{3} \mathrm{H}(1 ; z)\right)+z\left(-\frac{3}{2}-\frac{7}{3} \mathrm{H}(0 ; z)-2 \mathrm{H}(1 ; z)\right)\right] \\
+\left[\frac{10}{9}\right. & +\frac{\pi^{2}}{2}+\delta(1-z)\left(-\frac{28613}{648}+\frac{25 \pi^{2}}{6}+\frac{118 \zeta_{3}}{9}\right)+\left(\frac{557}{18}-\frac{23 \pi^{2}}{9}\right) \mathcal{D}_{0}(z)-\frac{188}{9} \mathcal{D}_{1}(z)+\frac{20}{3} \mathcal{D}_{2}(z) \\
& -\frac{38}{9} \mathrm{H}(0 ; z)-\frac{8}{3} \mathrm{H}(1 ; z)-\frac{19}{3} \mathrm{H}(0,0 ; z)-\frac{14}{3} \mathrm{H}(0,1 ; z)-\frac{16}{3} \mathrm{H}(1,0 ; z)-4 \mathrm{H}(1,1 ; z) \\
& +\frac{1}{1-z}\left(22 \mathrm{H}(0 ; z)+\frac{50}{3} \mathrm{H}(0,0 ; z)+\frac{44}{3} \mathrm{H}(0,1 ; z)+\frac{44}{3} \mathrm{H}(1,0 ; z)\right)+\frac{1}{z}\left(-\frac{103}{9}+\frac{5 \pi^{2}}{9}\right. \\
& \left.-\frac{52}{9} \mathrm{H}(0 ; z)-\frac{52}{9} \mathrm{H}(1 ; z)-\frac{8}{3} \mathrm{H}(0,0 ; z)-\frac{8}{3} \mathrm{H}(0,1 ; z)-\frac{8}{3} \mathrm{H}(1,0 ; z)-\frac{8}{3} \mathrm{H}(1,1 ; z)\right) \\
& +z\left(-\frac{41}{12}+\frac{5 \pi^{2}}{6}-\frac{32}{9} \mathrm{H}(0 ; z)-3 \mathrm{H}(1 ; z)-\frac{16}{3} \mathrm{H}(0,0 ; z)-\frac{14}{3} \mathrm{H}(0,1 ; z)-\frac{13}{3} \mathrm{H}(1,0 ; z)\right. \\
& -4 \mathrm{H}(1,1 ; z))] \\
+ & \mathcal{O}(\epsilon)
\end{aligned}
$$

and at subleading colour

$$
\begin{aligned}
& \tilde{\mathcal{E}}_{q, q^{\prime} \bar{q}^{\prime} g}^{0}= \\
& +\frac{1}{\epsilon^{3}}\left[-\frac{2}{3} \delta(1-z)\right] \\
& +\frac{1}{\epsilon^{2}}\left[-\frac{19}{9} \delta(1-z)+\frac{4}{3} \mathcal{D}_{0}(z)-\frac{2}{3 z}\right] \\
& +\frac{1}{\epsilon}\left[\delta(1-z)\left(-\frac{373}{54}+\frac{5 \pi^{2}}{9}\right)+\frac{38}{9} \mathcal{D}_{0}(z)-\frac{8}{3} \mathcal{D}_{1}(z)+\frac{8 \mathrm{H}(0 ; z)}{3(1-z)}+\frac{1}{z}\left(-\frac{25}{9}-\frac{4}{3} \mathrm{H}(0 ; z)-\frac{4}{3} \mathrm{H}(1 ; z)\right)\right] \\
& +\left[+\delta(1-z)\left(-\frac{6973}{324}+\frac{95 \pi^{2}}{54}+\frac{64 \zeta_{3}}{9}\right)+\left(\frac{373}{27}-\frac{10 \pi^{2}}{9}\right) \mathcal{D}_{0}(z)-\frac{76}{9} \mathcal{D}_{1}(z)+\frac{8}{3} \mathcal{D}_{2}(z)+\frac{1}{1-z}\left(\frac{76}{9} \mathrm{H}(0 ; z)\right.\right. \\
& \left.\quad+\frac{16}{3} \mathrm{H}(0,0 ; z)+\frac{16}{3} \mathrm{H}(0,1 ; z)+\frac{16}{3} \mathrm{H}(1,0 ; z)\right)+\frac{1}{z}\left(-\frac{523}{54}+\frac{5 \pi^{2}}{9}\right. \\
& \left.\left.\quad-\frac{50}{9} \mathrm{H}(0 ; z)-\frac{50}{9} \mathrm{H}(1 ; z)-\frac{8}{3} \mathrm{H}(0,0 ; z)-\frac{8}{3} \mathrm{H}(0,1 ; z)-\frac{8}{3} \mathrm{H}(1,0 ; z)-\frac{8}{3} \mathrm{H}(1,1 ; z)\right)\right] \\
& +\mathcal{O}(\epsilon) .
\end{aligned}
$$

Crossing the secondary quark, one obtains the $\left(q^{\prime}, q^{\prime} q g\right)$ antenna functions, whose integral reads at leading colour

$$
\begin{aligned}
& \mathcal{E}_{q^{\prime}, q^{\prime} q g}^{0}= \\
& +\frac{1}{\epsilon^{3}}\left[-4+\frac{4}{z}+2 z\right] \\
& +\frac{1}{\epsilon^{2}}\left[-15-\frac{4 z^{2}}{3}-4 \mathrm{H}(0 ; z)-8 \mathrm{H}(1 ; z)+\frac{1}{z}\left(\frac{55}{3}+8 \mathrm{H}(0 ; z)+8 \mathrm{H}(1 ; z)\right)\right. \\
& \left.\quad+z\left(\frac{3}{2}+\frac{13}{2} \mathrm{H}(0 ; z)+4 \mathrm{H}(1 ; z)\right)\right] \\
& +\frac{1}{\epsilon}\left[-\frac{235}{6}+\frac{7 \pi^{2}}{3}-39 \mathrm{H}(0 ; z)-30 \mathrm{H}(1 ; z)+4 \mathrm{H}(-1,0 ; z)-8 \mathrm{H}(0,0 ; z)-8 \mathrm{H}(0,1 ; z)\right.
\end{aligned}
$$




$$
\begin{aligned}
& -16 \mathrm{H}(1,0 ; z)-16 \mathrm{H}(1,1 ; z)+\frac{1}{z}\left(\frac{1537}{36}-3 \pi^{2}+28 \mathrm{H}(0 ; z)+\frac{110}{3} \mathrm{H}(1 ; z)+4 \mathrm{H}(-1,0 ; z)\right. \\
& +16 \mathrm{H}(0,0 ; z)+16 \mathrm{H}(0,1 ; z)+16 \mathrm{H}(1,0 ; z)+16 \mathrm{H}(1,1 ; z))+z\left(\frac{47}{12}-\frac{7 \pi^{2}}{3}-\frac{5}{2} \mathrm{H}(0 ; z)\right. \\
& \left.+3 \mathrm{H}(1 ; z)+2 \mathrm{H}(-1,0 ; z)+\frac{31}{2} \mathrm{H}(0,0 ; z)+13 \mathrm{H}(0,1 ; z)+8 \mathrm{H}(1,0 ; z)+8 \mathrm{H}(1,1 ; z)\right) \\
& \left.+z^{2}\left(\frac{32}{9}-\frac{16}{3} \mathrm{H}(0 ; z)-\frac{8}{3} \mathrm{H}(1 ; z)\right)\right] \\
& +\left[-\frac{2695}{18}+\frac{115 \pi^{2}}{6}+\frac{170 \zeta_{3}}{3}-2 \pi^{2} \mathrm{H}(-1 ; z)-\frac{337}{6} \mathrm{H}(0 ; z)+\frac{14}{3} \pi^{2} \mathrm{H}(0 ; z)-\frac{235}{3} \mathrm{H}(1 ; z)\right. \\
& +\frac{20}{3} \pi^{2} \mathrm{H}(1 ; z)-89 \mathrm{H}(0,0 ; z)-78 \mathrm{H}(0,1 ; z)-52 \mathrm{H}(1,0 ; z)-60 \mathrm{H}(1,1 ; z) \\
& -8 \mathrm{H}(-1,-1,0 ; z)+16 \mathrm{H}(-1,0,0 ; z)+8 \mathrm{H}(-1,0,1 ; z)+8 \mathrm{H}(0,-1,0 ; z)-16 \mathrm{H}(0,0,0 ; z) \\
& -16 \mathrm{H}(0,0,1 ; z)-12 \mathrm{H}(0,1,0 ; z)-16 \mathrm{H}(0,1,1 ; z)-32 \mathrm{H}(1,0,0 ; z)-32 \mathrm{H}(1,0,1 ; z) \\
& -32 \mathrm{H}(1,1,0 ; z)-32 \mathrm{H}(1,1,1 ; z)+\frac{1}{z}\left(\frac{37925}{216}-\frac{91 \pi^{2}}{6}-\frac{134 \zeta_{3}}{3}-2 \pi^{2} \mathrm{H}(-1 ; z)\right. \\
& +\frac{193}{2} \mathrm{H}(0 ; z)-8 \pi^{2} \mathrm{H}(0 ; z)+\frac{1537}{18} \mathrm{H}(1 ; z)-\frac{20}{3} \pi^{2} \mathrm{H}(1 ; z)+\frac{4}{3} \mathrm{H}(-1,0 ; z)+56 \mathrm{H}(0,0 ; z) \\
& +56 \mathrm{H}(0,1 ; z)+\frac{205}{3} \mathrm{H}(1,0 ; z)+\frac{220}{3} \mathrm{H}(1,1 ; z)-8 \mathrm{H}(-1,-1,0 ; z)+16 \mathrm{H}(-1,0,0 ; z) \\
& +8 \mathrm{H}(-1,0,1 ; z)+8 \mathrm{H}(0,-1,0 ; z)+32 \mathrm{H}(0,0,0 ; z)+32 \mathrm{H}(0,0,1 ; z)+28 \mathrm{H}(0,1,0 ; z) \\
& +32 \mathrm{H}(0,1,1 ; z)+32 \mathrm{H}(1,0,0 ; z)+32 \mathrm{H}(1,0,1 ; z)+32 \mathrm{H}(1,1,0 ; z)+32 \mathrm{H}(1,1,1 ; z)) \\
& +z\left(\frac{1069}{72}-\frac{3 \pi^{2}}{4}-\frac{82 \zeta_{3}}{3}-\pi^{2} \mathrm{H}(-1 ; z)+22 \mathrm{H}(0 ; z)-\frac{83}{12} \pi^{2} \mathrm{H}(0 ; z)+\frac{47}{6} \mathrm{H}(1 ; z)\right. \\
& -\frac{10}{3} \pi^{2} \mathrm{H}(1 ; z)-4 \mathrm{H}(-1,0 ; z)-\frac{23}{2} \mathrm{H}(0,0 ; z)-5 \mathrm{H}(0,1 ; z)+3 \mathrm{H}(1,0 ; z)+6 \mathrm{H}(1,1 ; z) \\
& -4 \mathrm{H}(-1,-1,0 ; z)+8 \mathrm{H}(-1,0,0 ; z)+4 \mathrm{H}(-1,0,1 ; z)+4 \mathrm{H}(0,-1,0 ; z)+\frac{67}{2} \mathrm{H}(0,0,0 ; z) \\
& +31 \mathrm{H}(0,0,1 ; z)+24 \mathrm{H}(0,1,0 ; z)+26 \mathrm{H}(0,1,1 ; z)+16 \mathrm{H}(1,0,0 ; z)+16 \mathrm{H}(1,0,1 ; z) \\
& +16 \mathrm{H}(1,1,0 ; z)+16 \mathrm{H}(1,1,1 ; z))+z^{2}\left(-\frac{208}{27}+2 \pi^{2}+\frac{128}{9} \mathrm{H}(0 ; z)+\frac{64}{9} \mathrm{H}(1 ; z)\right. \\
& \left.\left.-\frac{8}{3} \mathrm{H}(-1,0 ; z)-\frac{40}{3} \mathrm{H}(0,0 ; z)-\frac{32}{3} \mathrm{H}(0,1 ; z)-\frac{16}{3} \mathrm{H}(1,0 ; z)-\frac{16}{3} \mathrm{H}(1,1 ; z)\right)\right] \\
& +\mathcal{O}(\epsilon)
\end{aligned}
$$

and at subleading colour

$$
\begin{aligned}
& \tilde{\mathcal{E}}_{q^{\prime}, q^{\prime} q g}^{0}= \\
+ & \frac{1}{\epsilon^{3}}\left[-2+\frac{2}{z}+z\right] \\
+ & \frac{1}{\epsilon^{2}}\left[-8-5 \mathrm{H}(0 ; z)-4 \mathrm{H}(1 ; z)+\frac{1}{z}(9+4 \mathrm{H}(0 ; z)+4 \mathrm{H}(1 ; z))+z\left(\frac{7}{4}+\frac{5}{2} \mathrm{H}(0 ; z)+2 \mathrm{H}(1 ; z)\right)\right] \\
+ & \frac{1}{\epsilon}\left[-\frac{127}{4}+2 \pi^{2}-16 \mathrm{H}(0 ; z)-16 \mathrm{H}(1 ; z)-11 \mathrm{H}(0,0 ; z)-10 \mathrm{H}(0,1 ; z)-8 \mathrm{H}(1,0 ; z)\right. \\
& \quad-8 \mathrm{H}(1,1 ; z)+\frac{1}{z}\left(\frac{75}{2}-\frac{5 \pi^{2}}{3}+18 \mathrm{H}(0 ; z)+18 \mathrm{H}(1 ; z)+8 \mathrm{H}(0,0 ; z)+8 \mathrm{H}(0,1 ; z)\right.
\end{aligned}
$$




$$
\begin{aligned}
& +8 \mathrm{H}(1,0 ; z)+8 \mathrm{H}(1,1 ; z))+z\left(\frac{7}{2}-\pi^{2}+\frac{19}{4} \mathrm{H}(0 ; z)+\frac{7}{2} \mathrm{H}(1 ; z)+\frac{11}{2} \mathrm{H}(0,0 ; z)\right. \\
& +5 \mathrm{H}(0,1 ; z)+4 \mathrm{H}(1,0 ; z)+4 \mathrm{H}(1,1 ; z))] \\
& +\left[-\frac{499}{4}+\frac{20 \pi^{2}}{3}+\frac{70 \zeta_{3}}{3}-\frac{259}{4} \mathrm{H}(0 ; z)+\frac{9}{2} \pi^{2} \mathrm{H}(0 ; z)-\frac{127}{2} \mathrm{H}(1 ; z)+\frac{10}{3} \pi^{2} \mathrm{H}(1 ; z)\right. \\
& +32 \mathrm{H}(0,0 ; z)-32 \mathrm{H}(0,1 ; z)-32 \mathrm{H}(1,0 ; z)-32 \mathrm{H}(1,1 ; z)-23 \mathrm{H}(0,0,0 ; z) \\
& +22 \mathrm{H}(0,0,1 ; z)-20 \mathrm{H}(0,1,0 ; z)-20 \mathrm{H}(0,1,1 ; z)-16 \mathrm{H}(1,0,0 ; z)-16 \mathrm{H}(1,0,1 ; z) \\
& +16 \mathrm{H}(1,1,0 ; z)-16 \mathrm{H}(1,1,1 ; z)+\frac{1}{z}\left(\frac{565}{4}-\frac{15 \pi^{2}}{2}+75 \mathrm{H}(0 ; z)-\frac{10}{3} \pi^{2} \mathrm{H}(0 ; z)\right. \\
& +75 \mathrm{H}(1 ; z)-\frac{10}{3} \pi^{2} \mathrm{H}(1 ; z)+36 \mathrm{H}(0,0 ; z)+36 \mathrm{H}(0,1 ; z)+36 \mathrm{H}(1,0 ; z)+36 \mathrm{H}(1,1 ; z) \\
& +16 \mathrm{H}(0,0,0 ; z)+16 \mathrm{H}(0,0,1 ; z)+16 \mathrm{H}(0,1,0 ; z)+16 \mathrm{H}(0,1,1 ; z)+16 \mathrm{H}(1,0,0 ; z) \\
& \left.+16 \mathrm{H}(1,0,1 ; z)+16 \mathrm{H}(1,1,0 ; z)+16 \mathrm{H}(1,1,1 ; z)-\frac{64 \zeta_{3}}{3}\right)+z\left(13-\frac{15 \pi^{2}}{8}+7 \mathrm{H}(0 ; z)\right. \\
& +\frac{9}{4} \pi^{2} \mathrm{H}(0 ; z)+7 \mathrm{H}(1 ; z)-\frac{5}{3} \pi^{2} \mathrm{H}(1 ; z)+\frac{43}{4} \mathrm{H}(0,0 ; z)+\frac{19}{2} \mathrm{H}(0,1 ; z)+7 \mathrm{H}(1,0 ; z) \\
& +7 \mathrm{H}(1,1 ; z)+\frac{23}{2} \mathrm{H}(0,0,0 ; z)+11 \mathrm{H}(0,0,1 ; z)+10 \mathrm{H}(0,1,0 ; z)+10 \mathrm{H}(0,1,1 ; z) \\
& \left.\left.+8 \mathrm{H}(1,0,0 ; z)+8 \mathrm{H}(1,0,1 ; z)+8 \mathrm{H}(1,1,0 ; z)+8 \mathrm{H}(1,1,1 ; z)-\frac{35 \zeta_{3}}{3}\right)\right] \\
& +
\end{aligned}
$$

The one-loop virtual antenna functions at NNLO are obtained by crossing the results from [65]. For the $(q, g g)$-case, we have the leading colour and the quark loop correction, which integrate to

$$
\begin{aligned}
& \mathcal{D}_{q, g g}^{1}= \\
&+\frac{1}{\epsilon^{4}}\left[-\frac{1}{2} \delta(1-z)\right] \\
&+\frac{1}{\epsilon^{3}}\left[-\frac{1}{2}-\frac{55}{12} \delta(1-z)+\mathcal{D}_{0}(z)-\frac{z}{2}\right] \\
&+\frac{1}{\epsilon^{2}}\left[-\frac{7}{3}+\delta(1-z)\left(-\frac{85}{12}+\frac{7 \pi^{2}}{12}\right)+\frac{11}{2} \mathcal{D}_{0}(z)-2 \mathcal{D}_{1}(z)-2 \mathrm{H}(0 ; z)-\mathrm{H}(1 ; z)+\frac{4 \mathrm{H}(0 ; z)}{1-z}+\frac{1}{3 z}\right. \\
&\left.+\frac{1}{\epsilon}\left[-\frac{4}{3}-2 \mathrm{H}(0 ; z)-\mathrm{H}(1 ; z)\right)\right] \\
& \quad-\frac{23}{6} \mathrm{H}(0 ; z)-\frac{17}{6} \mathrm{H}(1 ; z)-6 \mathrm{H}(0,0 ; z)-3 \mathrm{H}(0,1 ; z)-3 \mathrm{H}(1,0 ; z)-2 \mathrm{H}(1,1 ; z) \\
& \quad+\frac{1}{1-z}(11 \mathrm{H}(0 ; z)+12 \mathrm{H}(0,0 ; z)+6 \mathrm{H}(0,1 ; z)+6 \mathrm{H}(1,0 ; z))+\frac{1}{z}\left(\frac{14}{9}+\frac{4}{3} \mathrm{H}(0 ; z)\right. \\
&\left.\quad+\frac{2}{3} \mathrm{H}(1 ; z)\right)+z\left(\frac{11}{6}+\frac{7 \pi^{2}}{12}+\frac{1}{6} \mathrm{H}(0 ; z)-\frac{5}{6} \mathrm{H}(1 ; z)-6 \mathrm{H}(0,0 ; z)-3 \mathrm{H}(0,1 ; z)\right. \\
&\quad-3 \mathrm{H}(1,0 ; z)-2 \mathrm{H}(1,1 ; z))]
\end{aligned}
$$




$$
\begin{aligned}
+[ & -\frac{17}{3}+\frac{67 \pi^{2}}{72}+\frac{25 \zeta_{3}}{3}+\delta(1-z)\left(-\frac{14453}{324}+\frac{3023 \pi^{2}}{432}-\frac{11 \pi^{4}}{72}+\frac{269 \zeta_{3}}{6}\right)+\left(\frac{3197}{108}-\frac{52 \pi^{2}}{9}\right. \\
& \left.-\frac{44 \zeta_{3}}{3}\right) \mathcal{D}_{0}(z)+\left(-\frac{73}{4}+\frac{7 \pi^{2}}{3}\right) \mathcal{D}_{1}(z)+\frac{11}{2} \mathcal{D}_{2}(z)-\frac{4}{3} \mathcal{D}_{3}(z)-\frac{9}{2} \mathrm{H}(0 ; z)+\frac{7}{3} \pi^{2} \mathrm{H}(0 ; z)-\frac{23}{6} \mathrm{H}(1 ; z) \\
& +\frac{4}{3} \pi^{2} \mathrm{H}(1 ; z)-\frac{79}{6} \mathrm{H}(0,0 ; z)-\frac{29}{6} \mathrm{H}(0,1 ; z)-\frac{61}{6} \mathrm{H}(1,0 ; z)-\frac{23}{6} \mathrm{H}(1,1 ; z) \\
& -14 \mathrm{H}(0,0,0 ; z)-8 \mathrm{H}(0,0,1 ; z)-6 \mathrm{H}(0,1,0 ; z)-5 \mathrm{H}(0,1,1 ; z)-6 \mathrm{H}(1,0,0 ; z) \\
& -5 \mathrm{H}(1,0,1 ; z)-4 \mathrm{H}(1,1,0 ; z)-4 \mathrm{H}(1,1,1 ; z)+\frac{1}{1-z}\left(\frac{11 \pi^{2}}{18}-2 \zeta_{3}+\frac{925}{36} \mathrm{H}(0 ; z)\right. \\
& -\frac{14}{3} \pi^{2} \mathrm{H}(0 ; z)-\frac{1}{3} \pi^{2} \mathrm{H}(1 ; z)+\frac{88}{3} \mathrm{H}(0,0 ; z)+\frac{44}{3} \mathrm{H}(0,1 ; z)+\frac{55}{3} \mathrm{H}(1,0 ; z) \\
& +28 \mathrm{H}(0,0,0 ; z)+16 \mathrm{H}(0,0,1 ; z)+12 \mathrm{H}(0,1,0 ; z)+10 \mathrm{H}(0,1,1 ; z)+12 \mathrm{H}(1,0,0 ; z) \\
& +10 \mathrm{H}(1,0,1 ; z)+8 \mathrm{H}(1,1,0 ; z))+\frac{1}{z}\left(\frac{145}{54}-\frac{11 \pi^{2}}{18}+\frac{53}{18} \mathrm{H}(0 ; z)+\frac{5}{2} \mathrm{H}(1 ; z)\right. \\
& \left.+\frac{10}{3} \mathrm{H}(0,0 ; z)+2 \mathrm{H}(0,1 ; z)+\frac{4}{3} \mathrm{H}(1,0 ; z)+\frac{4}{3} \mathrm{H}(1,1 ; z)\right)+z\left(\frac{79 \pi^{2}}{72}+\frac{25 \zeta_{3}}{3}+\frac{11}{6} \mathrm{H}(0 ; z)\right. \\
& +\frac{7}{3} \pi^{2} \mathrm{H}(0 ; z)+\frac{11}{6} \mathrm{H}(1 ; z)+\frac{4}{3} \pi^{2} \mathrm{H}(1 ; z)+\frac{17}{6} \mathrm{H}(0,0 ; z)+\frac{7}{6} \mathrm{H}(0,1 ; z)-\frac{1}{6} \mathrm{H}(1,0 ; z) \\
& +\frac{1}{6} \mathrm{H}(1,1 ; z)-14 \mathrm{H}(0,0,0 ; z)-8 \mathrm{H}(0,0,1 ; z)-6 \mathrm{H}(0,1,0 ; z)-5 \mathrm{H}(0,1,1 ; z) \\
& -6 \mathrm{H}(1,0,0 ; z)-5 \mathrm{H}(1,0,1 ; z)-4 \mathrm{H}(1,1,0 ; z)-4 \mathrm{H}(1,1,1 ; z))] \\
+ & \mathcal{O}(\epsilon)
\end{aligned}
$$

and

$$
\begin{aligned}
& \hat{\mathcal{D}}_{q, g g}^{1}= \\
& +\frac{1}{\epsilon^{3}}\left[\frac{2}{3} \delta(1-z)\right] \\
& +\frac{1}{\epsilon^{2}}\left[\frac{1}{3}+\frac{11}{18} \delta(1-z)-\frac{2}{3} \mathcal{D}_{0}(z)+\frac{z}{3}\right] \\
& +\frac{1}{\epsilon}\left[\frac{1}{3}+\delta(1-z)\left(\frac{143}{108}-\frac{\pi^{2}}{2}\right)-\frac{11}{18} \mathcal{D}_{0}(z)+\frac{2}{3} \mathcal{D}_{1}(z)+\frac{1}{3} \mathrm{H}(0 ; z)+\frac{1}{3} \mathrm{H}(1 ; z)-\frac{2 \mathrm{H}(0 ; z)}{3(1-z)}-\frac{1}{9 z}\right. \\
& \left.\quad+z\left(-\frac{1}{3}+\frac{1}{3} \mathrm{H}(0 ; z)+\frac{1}{3} \mathrm{H}(1 ; z)\right)\right] \\
& +\left[\frac{1}{2}-\frac{\pi^{2}}{4}+\delta(1-z)\left(\frac{979}{324}-\frac{11 \pi^{2}}{24}-\frac{14 \zeta_{3}}{9}\right)+\left(-\frac{38}{27}+\frac{\pi^{2}}{2}\right) \mathcal{D}_{0}(z)+\frac{11}{18} \mathcal{D}_{1}(z)-\frac{1}{3} \mathcal{D}_{2}(z)+\frac{1}{3} \mathrm{H}(0 ; z)\right. \\
& \quad+\frac{1}{3} \mathrm{H}(1 ; z)+\frac{1}{3} \mathrm{H}(0,0 ; z)+\frac{1}{3} \mathrm{H}(0,1 ; z)+\frac{1}{3} \mathrm{H}(1,0 ; z)+\frac{1}{3} \mathrm{H}(1,1 ; z)+\frac{1}{1-z}\left(-\frac{11}{18} \mathrm{H}(0 ; z)\right. \\
& \left.\quad-\frac{2}{3} \mathrm{H}(0,0 ; z)-\frac{2}{3} \mathrm{H}(0,1 ; z)-\frac{2}{3} \mathrm{H}(1,0 ; z)\right)+\frac{1}{z}\left(-\frac{2}{27}-\frac{1}{9} \mathrm{H}(0 ; z)-\frac{1}{9} \mathrm{H}(1 ; z)\right)+z\left(-\frac{\pi^{2}}{4}\right. \\
& \left.\left.\quad-\frac{1}{3} \mathrm{H}(0 ; z)-\frac{1}{3} \mathrm{H}(1 ; z)+\frac{1}{3} \mathrm{H}(0,0 ; z)+\frac{1}{3} \mathrm{H}(0,1 ; z)+\frac{1}{3} \mathrm{H}(1,0 ; z)+\frac{1}{3} \mathrm{H}(1,1 ; z)\right)\right] \\
& +
\end{aligned}
$$

For the $\left(q, q^{\prime} \bar{q}^{\prime}\right)$-case (primary quark in the initial state), there are leading and sub- 
leading colour as well as quark loop contributions. Their integrals are:

$$
\begin{aligned}
& \mathcal{E}_{q, q^{\prime} \bar{q}^{\prime}}^{1}= \\
& +\frac{1}{\epsilon^{2}}\left[\frac{11}{18} \delta(1-z)\right] \\
& +\frac{1}{\epsilon}\left[\frac{119}{108} \delta(1-z)-\frac{11}{18} \mathcal{D}_{0}(z)-\frac{2 \mathrm{H}(0 ; z)}{3(1-z)}+\frac{1}{z}\left(\frac{2}{9}+\frac{1}{3} \mathrm{H}(0 ; z)\right)\right] \\
& +\left[-\frac{1}{6}+\delta(1-z)\left(\frac{787}{324}-\frac{17 \pi^{2}}{24}\right)+\frac{2 \pi^{2}}{9}-\frac{32}{27} \mathcal{D}_{0}(z)+\frac{11}{18} \mathcal{D}_{1}(z)-\frac{1}{6} \mathrm{H}(0 ; z)+\frac{2}{3} \mathrm{H}(0,0 ; z)\right. \\
& \quad+\frac{2}{3} \mathrm{H}(1,0 ; z)+\frac{1}{1-z}\left(-\frac{\pi^{2}}{9}-\frac{31}{18} \mathrm{H}(0 ; z)-\frac{8}{3} \mathrm{H}(0,0 ; z)-\frac{2}{3} \mathrm{H}(0,1 ; z)-\frac{4}{3} \mathrm{H}(1,0 ; z)\right) \\
& \quad+\frac{1}{z}\left(\frac{119}{216}-\frac{\pi^{2}}{18}+\frac{37}{36} \mathrm{H}(0 ; z)-\frac{1}{9} \mathrm{H}(1 ; z)+\mathrm{H}(0,0 ; z)+\frac{1}{3} \mathrm{H}(0,1 ; z)+\frac{1}{3} \mathrm{H}(1,0 ; z)\right) \\
& \left.\quad+z\left(\frac{\pi^{2}}{18}+\frac{1}{6} \mathrm{H}(0,0 ; z)+\frac{1}{6} \mathrm{H}(1,0 ; z)\right)\right] \\
& +\mathcal{O}(\epsilon),
\end{aligned}
$$

$$
\begin{aligned}
& \tilde{\mathcal{E}}_{q, q^{\prime} \bar{q}^{\prime}}^{1}= \\
& +\frac{1}{\epsilon^{3}}\left[\frac{1}{6} \delta(1-z)\right] \\
& +\frac{1}{\epsilon^{2}}\left[\frac{19}{36} \delta(1-z)-\frac{1}{3} \mathcal{D}_{0}(z)+\frac{1}{6 z}\right] \\
& +\frac{1}{\epsilon}\left[\delta(1-z)\left(\frac{173}{108}-\frac{5 \pi^{2}}{36}\right)-\frac{19}{18} \mathcal{D}_{0}(z)+\frac{2}{3} \mathcal{D}_{1}(z)-\frac{2 \mathrm{H}(0 ; z)}{3(1-z)}+\frac{1}{z}\left(\frac{25}{36}+\frac{1}{3} \mathrm{H}(0 ; z)+\frac{1}{3} \mathrm{H}(1 ; z)\right)\right] \\
& +\left[\delta(1-z)\left(\frac{343}{81}-\frac{95 \pi^{2}}{216}-\frac{7 \zeta_{3}}{9}\right)+\left(-\frac{173}{54}+\frac{5 \pi^{2}}{18}\right) \mathcal{D}_{0}(z)+\frac{19}{9} \mathcal{D}_{1}(z)-\frac{2}{3} \mathcal{D}_{2}(z)+\frac{1}{1-z}\left(-\frac{19}{9} \mathrm{H}(0 ; z)\right.\right. \\
& \left.\quad-\frac{4}{3} \mathrm{H}(0,0 ; z)-\frac{4}{3} \mathrm{H}(0,1 ; z)-\frac{4}{3} \mathrm{H}(1,0 ; z)\right)+\frac{1}{z}\left(\frac{62}{27}-\frac{5 \pi^{2}}{36}+\frac{25}{18} \mathrm{H}(0 ; z)+\frac{25}{18} \mathrm{H}(1 ; z)\right. \\
& \left.\left.\quad+\frac{2}{3} \mathrm{H}(0,0 ; z)+\frac{2}{3} \mathrm{H}(0,1 ; z)+\frac{2}{3} \mathrm{H}(1,0 ; z)+\frac{2}{3} \mathrm{H}(1,1 ; z)\right)\right] \\
& +
\end{aligned}
$$

and

$$
\begin{aligned}
& \hat{\mathcal{E}}_{q, q^{\prime} \bar{q}^{\prime}}^{1}= \\
+ & \frac{1}{\epsilon}\left[\frac{5}{27} \delta(1-z)-\frac{1}{9} \mathcal{D}_{0}(z)+\frac{1}{18 z}\right] \\
+ & {\left[\delta(1-z)\left(\frac{53}{81}-\frac{\pi^{2}}{108}\right)-\frac{5}{9} \mathcal{D}_{0}(z)+\frac{1}{3} \mathcal{D}_{1}(z)-\frac{\mathrm{H}(0 ; z)}{3(1-z)}+\frac{1}{z}\left(\frac{1}{3}+\frac{1}{6} \mathrm{H}(0 ; z)+\frac{1}{6} \mathrm{H}(1 ; z)\right)\right] } \\
& +\mathcal{O}(\epsilon) .
\end{aligned}
$$

Likewise, the $\left(q^{\prime}, q^{\prime} q\right)$-case (secondary quark in the initial state), there are leading and subleading colour as well as quark loop contributions, which integrate to:

$$
\mathcal{E}_{q^{\prime}, q^{\prime} q}^{1}=
$$




$$
\begin{aligned}
& +\frac{1}{\epsilon^{2}}\left[\frac{3}{4}+\mathrm{H}(0 ; z)+\frac{1}{2} \mathrm{H}(1 ; z)+\frac{1}{z}\left(-\frac{3}{4}-\mathrm{H}(0 ; z)-\frac{1}{2} \mathrm{H}(1 ; z)\right)-z\left(\frac{3}{8}+\frac{1}{2} \mathrm{H}(0 ; z)+\frac{1}{4} \mathrm{H}(1 ; z)\right)\right] \\
& +\frac{1}{\epsilon}\left[-\frac{41}{36}-\frac{\pi^{2}}{3}+\frac{5}{3} \mathrm{H}(0 ; z)+\frac{7}{4} \mathrm{H}(1 ; z)+4 \mathrm{H}(0,0 ; z)+2 \mathrm{H}(0,1 ; z)+2 \mathrm{H}(1,0 ; z)+\frac{3}{2} \mathrm{H}(1,1 ; z)\right. \\
& +\frac{1}{z}\left(\frac{29}{36}+\frac{\pi^{2}}{3}-\frac{8}{3} \mathrm{H}(0 ; z)-\frac{9}{4} \mathrm{H}(1 ; z)-4 \mathrm{H}(0,0 ; z)-2 \mathrm{H}(0,1 ; z)-2 \mathrm{H}(1,0 ; z)\right. \\
& \left.-\frac{3}{2} \mathrm{H}(1,1 ; z)\right)+z\left(\frac{10}{9}+\frac{\pi^{2}}{6}+\frac{1}{6} \mathrm{H}(0 ; z)-\frac{3}{8} \mathrm{H}(1 ; z)-2 \mathrm{H}(0,0 ; z)-\mathrm{H}(0,1 ; z)\right. \\
& \left.\left.-\mathrm{H}(1,0 ; z)-\frac{3}{4} \mathrm{H}(1,1 ; z)\right)\right] \\
& +\left[-\frac{347}{54}-\frac{43 \pi^{2}}{12}-5 \zeta_{3}-\frac{1}{36} \mathrm{H}(0 ; z)-\frac{11}{6} \pi^{2} \mathrm{H}(0 ; z)+\frac{49}{36} \mathrm{H}(1 ; z)-\frac{3}{4} \pi^{2} \mathrm{H}(1 ; z)+\frac{3}{2} \mathrm{H}(0,0 ; z)\right. \\
& +\frac{11}{3} \mathrm{H}(0,1 ; z)-\frac{1}{3} \mathrm{H}(1,0 ; z)+\frac{15}{4} \mathrm{H}(1,1 ; z)+10 \mathrm{H}(0,0,0 ; z)+6 \mathrm{H}(0,0,1 ; z) \\
& +4 \mathrm{H}(0,1,0 ; z)+4 \mathrm{H}(0,1,1 ; z)+5 \mathrm{H}(1,0,0 ; z)+4 \mathrm{H}(1,0,1 ; z)+4 \mathrm{H}(1,1,0 ; z) \\
& +\frac{7}{2} \mathrm{H}(1,1,1 ; z)+\frac{1}{z}\left(\frac{871}{108}+\frac{41 \pi^{2}}{12}+5 \zeta_{3}-\frac{31}{18} \mathrm{H}(0 ; z)+\frac{11}{6} \pi^{2} \mathrm{H}(0 ; z)-\frac{115}{36} \mathrm{H}(1 ; z)\right. \\
& +\frac{3}{4} \pi^{2} \mathrm{H}(1 ; z)-\frac{15}{2} \mathrm{H}(0,0 ; z)-\frac{17}{3} \mathrm{H}(0,1 ; z)-\frac{19}{6} \mathrm{H}(1,0 ; z)-\frac{21}{4} \mathrm{H}(1,1 ; z) \\
& -10 \mathrm{H}(0,0,0 ; z)-6 \mathrm{H}(0,0,1 ; z)-4 \mathrm{H}(0,1,0 ; z)-4 \mathrm{H}(0,1,1 ; z)-5 \mathrm{H}(1,0,0 ; z) \\
& \left.-4 \mathrm{H}(1,0,1 ; z)-4 \mathrm{H}(1,1,0 ; z)-\frac{7}{2} \mathrm{H}(1,1,1 ; z)\right)+z\left(\frac{121}{54}+\frac{9 \pi^{2}}{8}+\frac{5 \zeta_{3}}{2}+\frac{20}{9} \mathrm{H}(0 ; z)\right. \\
& +\frac{11}{12} \pi^{2} \mathrm{H}(0 ; z)+\frac{10}{9} \mathrm{H}(1 ; z)+\frac{3}{8} \pi^{2} \mathrm{H}(1 ; z)+\frac{9}{4} \mathrm{H}(0,0 ; z)+\frac{1}{6} \mathrm{H}(0,1 ; z)+\frac{7}{6} \mathrm{H}(1,0 ; z) \\
& -\frac{3}{8} \mathrm{H}(1,1 ; z)-5 \mathrm{H}(0,0,0 ; z)-3 \mathrm{H}(0,0,1 ; z)-2 \mathrm{H}(0,1,0 ; z)-2 \mathrm{H}(0,1,1 ; z) \\
& \left.\left.-\frac{5}{2} \mathrm{H}(1,0,0 ; z)-2 \mathrm{H}(1,0,1 ; z)-2 \mathrm{H}(1,1,0 ; z)-\frac{7}{4} \mathrm{H}(1,1,1 ; z)\right)\right] \\
& +\mathcal{O}(\epsilon) \text {, } \\
& \tilde{\mathcal{E}}_{q^{\prime}, q^{\prime} q}^{1}= \\
& +\frac{1}{\epsilon^{3}}\left[\frac{1}{2}-\frac{1}{2 z}-\frac{z}{4}\right] \\
& +\frac{1}{\epsilon^{2}}\left[\frac{7}{4}+\mathrm{H}(0 ; z)+\frac{1}{2} \mathrm{H}(1 ; z)-\frac{1}{z}\left(\frac{9}{4}+\mathrm{H}(0 ; z)+\frac{1}{2} \mathrm{H}(1 ; z)\right)-z\left(\frac{3}{8}+\frac{1}{2} \mathrm{H}(0 ; z)+\frac{1}{4} \mathrm{H}(1 ; z)\right)\right] \\
& +\frac{1}{\epsilon}\left[\frac{13}{2}-\frac{\pi^{2}}{4}+\frac{7}{2} \mathrm{H}(0 ; z)+\frac{7}{4} \mathrm{H}(1 ; z)+2 \mathrm{H}(0,0 ; z)+\mathrm{H}(0,1 ; z)+\mathrm{H}(1,0 ; z)+\frac{1}{2} \mathrm{H}(1,1 ; z)\right. \\
& +\frac{1}{z}\left(-\frac{35}{4}+\frac{\pi^{2}}{4}-\frac{9}{2} \mathrm{H}(0 ; z)-\frac{9}{4} \mathrm{H}(1 ; z)-2 \mathrm{H}(0,0 ; z)-\mathrm{H}(0,1 ; z)-\mathrm{H}(1,0 ; z)\right. \\
& \left.-\frac{1}{2} \mathrm{H}(1,1 ; z)\right)+z\left(-1+\frac{\pi^{2}}{8}-\frac{3}{4} \mathrm{H}(0 ; z)-\frac{3}{8} \mathrm{H}(1 ; z)-\mathrm{H}(0,0 ; z)-\frac{1}{2} \mathrm{H}(0,1 ; z)\right. \\
& \left.\left.-\frac{1}{2} \mathrm{H}(1,0 ; z)-\frac{1}{4} \mathrm{H}(1,1 ; z)\right)\right] \\
& +\left[\frac{43}{2}-\frac{7 \pi^{2}}{8}-\frac{13 \zeta_{3}}{3}+13 \mathrm{H}(0 ; z)-\frac{1}{2} \pi^{2} \mathrm{H}(0 ; z)+\frac{13}{2} \mathrm{H}(1 ; z)-\frac{1}{4} \pi^{2} \mathrm{H}(1 ; z)+7 \mathrm{H}(0,0 ; z)\right. \\
& +\frac{7}{2} \mathrm{H}(0,1 ; z)+\frac{7}{2} \mathrm{H}(1,0 ; z)+\frac{7}{4} \mathrm{H}(1,1 ; z)+4 \mathrm{H}(0,0,0 ; z)+2 \mathrm{H}(0,0,1 ; z)+2 \mathrm{H}(0,1,0 ; z)
\end{aligned}
$$




$$
\begin{aligned}
& +\mathrm{H}(0,1,1 ; z)+2 \mathrm{H}(1,0,0 ; z)+\mathrm{H}(1,0,1 ; z)+\mathrm{H}(1,1,0 ; z)+\frac{1}{2} \mathrm{H}(1,1,1 ; z)+\frac{1}{z}\left(-\frac{121}{4}\right. \\
& +\frac{9 \pi^{2}}{8}+\frac{13 \zeta_{3}}{3}-\frac{35}{2} \mathrm{H}(0 ; z)+\frac{1}{2} \pi^{2} \mathrm{H}(0 ; z)-\frac{35}{4} \mathrm{H}(1 ; z)+\frac{1}{4} \pi^{2} \mathrm{H}(1 ; z)-9 \mathrm{H}(0,0 ; z) \\
& -\frac{9}{2} \mathrm{H}(0,1 ; z)-\frac{9}{2} \mathrm{H}(1,0 ; z)-\frac{9}{4} \mathrm{H}(1,1 ; z)-4 \mathrm{H}(0,0,0 ; z)-2 \mathrm{H}(0,0,1 ; z)-2 \mathrm{H}(0,1,0 ; z) \\
& \left.-\mathrm{H}(0,1,1 ; z)-2 \mathrm{H}(1,0,0 ; z)-\mathrm{H}(1,0,1 ; z)-\mathrm{H}(1,1,0 ; z)-\frac{1}{2} \mathrm{H}(1,1,1 ; z)\right)+z(-2 \\
& +\frac{3 \pi^{2}}{16}+\frac{13 \zeta_{3}}{6}-2 \mathrm{H}(0 ; z)+\frac{1}{4} \pi^{2} \mathrm{H}(0 ; z)-\mathrm{H}(1 ; z)+\frac{1}{8} \pi^{2} \mathrm{H}(1 ; z)-\frac{3}{2} \mathrm{H}(0,0 ; z) \\
& -\frac{3}{4} \mathrm{H}(0,1 ; z)-\frac{3}{4} \mathrm{H}(1,0 ; z)-\frac{3}{8} \mathrm{H}(1,1 ; z)-2 \mathrm{H}(0,0,0 ; z)-\mathrm{H}(0,0,1 ; z)-\mathrm{H}(0,1,0 ; z) \\
& \left.\left.-\frac{1}{2} \mathrm{H}(0,1,1 ; z)-\mathrm{H}(1,0,0 ; z)-\frac{1}{2} \mathrm{H}(1,0,1 ; z)-\frac{1}{2} \mathrm{H}(1,1,0 ; z)-\frac{1}{4} \mathrm{H}(1,1,1 ; z)\right)\right] \\
& +\mathcal{O}(\epsilon)
\end{aligned}
$$

and

$$
\begin{aligned}
& \hat{\mathcal{E}}_{q^{\prime}, q^{\prime} q}^{1}= \\
& +\frac{1}{\epsilon}\left[\frac{13}{18}+\frac{1}{3} \mathrm{H}(0 ; z)-\frac{1}{z}\left(\frac{8}{9}+\frac{1}{3} \mathrm{H}(0 ; z)\right)-z\left(\frac{5}{18}+\frac{1}{6} \mathrm{H}(0 ; z)\right)\right] \\
& +\left[\frac{85}{27}+\frac{\pi^{2}}{12}+\frac{35}{18} \mathrm{H}(0 ; z)+\frac{13}{18} \mathrm{H}(1 ; z)+\mathrm{H}(0,0 ; z)+\frac{1}{3} \mathrm{H}(0,1 ; z)+\frac{1}{3} \mathrm{H}(1,0 ; z)+\frac{1}{z}\left(-\frac{118}{27}\right.\right. \\
& \left.\quad-\frac{\pi^{2}}{12}-\frac{22}{9} \mathrm{H}(0 ; z)-\frac{8}{9} \mathrm{H}(1 ; z)-\mathrm{H}(0,0 ; z)-\frac{1}{3} \mathrm{H}(0,1 ; z)-\frac{1}{3} \mathrm{H}(1,0 ; z)\right) \\
& \left.\quad+z\left(-\frac{14}{27}-\frac{\pi^{2}}{24}-\frac{5}{9} \mathrm{H}(0 ; z)-\frac{5}{18} \mathrm{H}(1 ; z)-\frac{1}{2} \mathrm{H}(0,0 ; z)-\frac{1}{6} \mathrm{H}(0,1 ; z)-\frac{1}{6} \mathrm{H}(1,0 ; z)\right)\right] \\
& \quad+\mathcal{O}(\epsilon) .
\end{aligned}
$$

\subsection{Gluon-gluon antennae}

The gluon-gluon antenna functions can contribute to quark-initiated processes, since they also contain configurations with the splitting of a gluon into a quark-antiquark pair.

At NLO, one has only the $(q, q g)$ gluon-gluon antenna function, whose integral is

$$
\begin{aligned}
& \mathcal{G}_{q, q g}^{0}= \\
& \quad \frac{1}{\epsilon}\left[-1+\frac{1}{z}+\frac{z}{2}\right] \\
& +\left[-1-\mathrm{H}(0 ; z)-\mathrm{H}(1 ; z)+\frac{1}{z}\left(\frac{7}{4}+\mathrm{H}(0 ; z)+\mathrm{H}(1 ; z)\right)+z\left(\frac{1}{2} \mathrm{H}(0 ; z)+\frac{1}{2} \mathrm{H}(1 ; z)\right)\right] \\
& +\epsilon\left[-2+\frac{\pi^{2}}{4}-\mathrm{H}(0 ; z)-\mathrm{H}(1 ; z)-\mathrm{H}(0,0 ; z)-\mathrm{H}(0,1 ; z)-\mathrm{H}(1,0 ; z)-\mathrm{H}(1,1 ; z)\right. \\
& \quad+\frac{1}{z}\left(\frac{7}{2}-\frac{\pi^{2}}{4}+\frac{7}{4} \mathrm{H}(0 ; z)+\frac{7}{4} \mathrm{H}(1 ; z)+\mathrm{H}(0,0 ; z)+\mathrm{H}(0,1 ; z)+\mathrm{H}(1,0 ; z)+\mathrm{H}(1,1 ; z)\right) \\
& \left.\quad+z\left(-\frac{\pi^{2}}{8}+\frac{1}{2} \mathrm{H}(0,0 ; z)+\frac{1}{2} \mathrm{H}(0,1 ; z)+\frac{1}{2} \mathrm{H}(1,0 ; z)+\frac{1}{2} \mathrm{H}(1,1 ; z)\right)\right]
\end{aligned}
$$




$$
\begin{aligned}
+\epsilon^{2}[ & -4+\frac{\pi^{2}}{4}+\frac{7 \zeta_{3}}{3}-2 \mathrm{H}(0 ; z)+\frac{1}{4} \pi^{2} \mathrm{H}(0 ; z)-2 \mathrm{H}(1 ; z)+\frac{1}{4} \pi^{2} \mathrm{H}(1 ; z)-\mathrm{H}(0,0 ; z) \\
& -\mathrm{H}(0,1 ; z)-\mathrm{H}(1,0 ; z)-\mathrm{H}(1,1 ; z)-\mathrm{H}(0,0,0 ; z)-\mathrm{H}(0,0,1 ; z)-\mathrm{H}(0,1,0 ; z) \\
& -\mathrm{H}(0,1,1 ; z)-\mathrm{H}(1,0,0 ; z)-\mathrm{H}(1,0,1 ; z)-\mathrm{H}(1,1,0 ; z)-\mathrm{H}(1,1,1 ; z)+\frac{1}{z}\left(7-\frac{7 \pi^{2}}{16}\right. \\
& -\frac{7 \zeta_{3}}{3}+\frac{7}{2} \mathrm{H}(0 ; z)-\frac{1}{4} \pi^{2} \mathrm{H}(0 ; z)+\frac{7}{2} \mathrm{H}(1 ; z)-\frac{1}{4} \pi^{2} \mathrm{H}(1 ; z)+\frac{7}{4} \mathrm{H}(0,0 ; z)+\frac{7}{4} \mathrm{H}(0,1 ; z) \\
& +\frac{7}{4} \mathrm{H}(1,0 ; z)+\frac{7}{4} \mathrm{H}(1,1 ; z)+\mathrm{H}(0,0,0 ; z)+\mathrm{H}(0,0,1 ; z)+\mathrm{H}(0,1,0 ; z)+\mathrm{H}(0,1,1 ; z) \\
& +\mathrm{H}(1,0,0 ; z)+\mathrm{H}(1,0,1 ; z)+\mathrm{H}(1,1,0 ; z)+\mathrm{H}(1,1,1 ; z))+z\left(-\frac{7 \zeta_{3}}{6}-\frac{1}{8} \pi^{2} \mathrm{H}(0 ; z)\right. \\
& -\frac{1}{8} \pi^{2} \mathrm{H}(1 ; z)+\frac{1}{2} \mathrm{H}(0,0,0 ; z)+\frac{1}{2} \mathrm{H}(0,0,1 ; z)+\frac{1}{2} \mathrm{H}(0,1,0 ; z)+\frac{1}{2} \mathrm{H}(0,1,1 ; z) \\
& \left.\left.+\frac{1}{2} \mathrm{H}(1,0,0 ; z)+\frac{1}{2} \mathrm{H}(1,0,1 ; z)+\frac{1}{2} \mathrm{H}(1,1,0 ; z)+\frac{1}{2} \mathrm{H}(1,1,1 ; z)\right)\right] \\
+ & \mathcal{O}\left(\epsilon^{3}\right) .
\end{aligned}
$$

At NNLO, the double real radiation $G_{4}^{0}$-type and $H_{4}^{0}=$ type antenna functions allow for crossings with initial state quarks.

The $(q, q g g)$ at leading colour integrates to

$$
\begin{aligned}
\mathcal{G}_{q, q g g}^{0}= \\
+\frac{1}{\epsilon^{3}}\left[-8+\frac{8}{z}+4 z\right] \\
+\frac{1}{\epsilon^{2}}\left[-\frac{82}{3}-9 \mathrm{H}(0 ; z)-16 \mathrm{H}(1 ; z)+\frac{1}{z}\left(\frac{104}{3}+16 \mathrm{H}(0 ; z)+16 \mathrm{H}(1 ; z)\right)+z\left(\frac{53}{12}+\frac{21}{2} \mathrm{H}(0 ; z)\right.\right. \\
\left.\quad+8 \mathrm{H}(1 ; z))-\frac{4}{3} z^{2}\right] \\
+\frac{1}{\epsilon}\left[-\frac{2291}{36}+\frac{13 \pi^{2}}{3}-\frac{185}{3} \mathrm{H}(0 ; z)-\frac{164}{3} \mathrm{H}(1 ; z)+4 \mathrm{H}(-1,0 ; z)-15 \mathrm{H}(0,0 ; z)\right. \\
\quad-18 \mathrm{H}(0,1 ; z)-32 \mathrm{H}(1,0 ; z)-32 \mathrm{H}(1,1 ; z)+\frac{1}{z}\left(\frac{499}{6}-6 \pi^{2}+\frac{164}{3} \mathrm{H}(0 ; z)+\frac{208}{3} \mathrm{H}(1 ; z)\right. \\
\quad+4 \mathrm{H}(-1,0 ; z)+32 \mathrm{H}(0,0 ; z)+32 \mathrm{H}(0,1 ; z)+32 \mathrm{H}(1,0 ; z)+32 \mathrm{H}(1,1 ; z))+z\left(\frac{301}{36}\right. \\
\quad-\frac{23 \pi^{2}}{6}+\frac{61}{12} \mathrm{H}(0 ; z)+\frac{53}{6} \mathrm{H}(1 ; z)+2 \mathrm{H}(-1,0 ; z)+\frac{47}{2} \mathrm{H}(0,0 ; z)+21 \mathrm{H}(0,1 ; z) \\
\left.+\quad 16 \mathrm{H}(1,0 ; z)+16 \mathrm{H}(1,1 ; z))+z^{2}\left(\frac{32}{9}-\frac{16}{3} \mathrm{H}(0 ; z)-\frac{8}{3} \mathrm{H}(1 ; z)\right)\right] \\
+\left[-\frac{6625}{27}+\frac{256 \pi^{2}}{9}+\frac{286 \zeta_{3}}{3}-2 \pi^{2} \mathrm{H}(-1 ; z)-\frac{3181}{36} \mathrm{H}(0 ; z)+\frac{55}{6} \pi^{2} \mathrm{H}(0 ; z)-\frac{2291}{18} \mathrm{H}(1 ; z)\right. \\
\quad+\frac{38}{3} \pi^{2} \mathrm{H}(1 ; z)-8 \mathrm{H}(-1,0 ; z)-\frac{373}{3} \mathrm{H}(0,0 ; z)-\frac{370}{3} \mathrm{H}(0,1 ; z)-\frac{298}{3} \mathrm{H}(1,0 ; z) \\
\quad-\frac{328}{3} \mathrm{H}(1,1 ; z)-8 \mathrm{H}(-1,-1,0 ; z)+16 \mathrm{H}(-1,0,0 ; z)+8 \mathrm{H}(-1,0,1 ; z)+16 \mathrm{H}(0,-1,0 ; z) \\
\quad-27 \mathrm{H}(0,0,0 ; z)-30 \mathrm{H}(0,0,1 ; z)-28 \mathrm{H}(0,1,0 ; z)-36 \mathrm{H}(0,1,1 ; z)-60 \mathrm{H}(1,0,0 ; z)
\end{aligned}
$$




$$
\begin{aligned}
& -64 \mathrm{H}(1,0,1 ; z)-64 \mathrm{H}(1,1,0 ; z)-64 \mathrm{H}(1,1,1 ; z)+\frac{1}{z}\left(\frac{33167}{108}-\frac{250 \pi^{2}}{9}-\frac{268 \zeta_{3}}{3}\right. \\
& -2 \pi^{2} \mathrm{H}(-1 ; z)+\frac{1633}{9} \mathrm{H}(0 ; z)-16 \pi^{2} \mathrm{H}(0 ; z)+\frac{499}{3} \mathrm{H}(1 ; z)-\frac{38}{3} \pi^{2} \mathrm{H}(1 ; z) \\
& -\frac{32}{3} \mathrm{H}(-1,0 ; z)+\frac{328}{3} \mathrm{H}(0,0 ; z)+\frac{328}{3} \mathrm{H}(0,1 ; z)+\frac{398}{3} \mathrm{H}(1,0 ; z)+\frac{416}{3} \mathrm{H}(1,1 ; z) \\
& -8 \mathrm{H}(-1,-1,0 ; z)+16 \mathrm{H}(-1,0,0 ; z)+8 \mathrm{H}(-1,0,1 ; z)+64 \mathrm{H}(0,0,0 ; z)+64 \mathrm{H}(0,0,1 ; z) \\
& +56 \mathrm{H}(0,1,0 ; z)+64 \mathrm{H}(0,1,1 ; z)+60 \mathrm{H}(1,0,0 ; z)+64 \mathrm{H}(1,0,1 ; z)+64 \mathrm{H}(1,1,0 ; z) \\
& +64 \mathrm{H}(1,1,1 ; z))+z\left(\frac{7411}{216}-\frac{199 \pi^{2}}{72}-\frac{149 \zeta_{3}}{3}-\pi^{2} \mathrm{H}(-1 ; z)+\frac{511}{18} \mathrm{H}(0 ; z)\right. \\
& -\frac{131}{12} \pi^{2} \mathrm{H}(0 ; z)+\frac{301}{18} \mathrm{H}(1 ; z)-\frac{19}{3} \pi^{2} \mathrm{H}(1 ; z)+\frac{65}{12} \mathrm{H}(0,0 ; z)+\frac{61}{6} \mathrm{H}(0,1 ; z) \\
& +\frac{50}{3} \mathrm{H}(1,0 ; z)+\frac{53}{3} \mathrm{H}(1,1 ; z)-4 \mathrm{H}(-1,-1,0 ; z)+8 \mathrm{H}(-1,0,0 ; z)+4 \mathrm{H}(-1,0,1 ; z) \\
& +\frac{99}{2} \mathrm{H}(0,0,0 ; z)+47 \mathrm{H}(0,0,1 ; z)+38 \mathrm{H}(0,1,0 ; z)+42 \mathrm{H}(0,1,1 ; z)+30 \mathrm{H}(1,0,0 ; z) \\
& +32 \mathrm{H}(1,0,1 ; z)+32 \mathrm{H}(1,1,0 ; z)+32 \mathrm{H}(1,1,1 ; z))+z^{2}\left(-\frac{208}{27}+2 \pi^{2}+\frac{128}{9} \mathrm{H}(0 ; z)\right. \\
& \left.\left.+\frac{64}{9} \mathrm{H}(1 ; z)-\frac{8}{3} \mathrm{H}(-1,0 ; z)-\frac{40}{3} \mathrm{H}(0,0 ; z)-\frac{32}{3} \mathrm{H}(0,1 ; z)-\frac{16}{3} \mathrm{H}(1,0 ; z)-\frac{16}{3} \mathrm{H}(1,1 ; z)\right)\right] \\
& + \\
& +
\end{aligned}
$$

while the subleading colour is

$$
\begin{aligned}
& \tilde{\mathcal{G}}_{q, q g g}^{0}= \\
& +\frac{1}{\epsilon^{3}}\left[-4+\frac{4}{z}+2 z\right] \\
& +\frac{1}{\epsilon^{2}}\left[-12-10 \mathrm{H}(0 ; z)-8 \mathrm{H}(1 ; z)+\frac{1}{z}(16+8 \mathrm{H}(0 ; z)+8 \mathrm{H}(1 ; z))+z\left(\frac{7}{2}+5 \mathrm{H}(0 ; z)+4 \mathrm{H}(1 ; z)\right)\right] \\
& +\frac{1}{\epsilon}\left[-\frac{91}{2}+4 \pi^{2}-22 \mathrm{H}(0 ; z)-24 \mathrm{H}(1 ; z)-22 \mathrm{H}(0,0 ; z)-20 \mathrm{H}(0,1 ; z)-16 \mathrm{H}(1,0 ; z)\right. \\
& -16 \mathrm{H}(1,1 ; z)+\frac{1}{z}\left(67-\frac{10 \pi^{2}}{3}+32 \mathrm{H}(0 ; z)+32 \mathrm{H}(1 ; z)+16 \mathrm{H}(0,0 ; z)+16 \mathrm{H}(0,1 ; z)\right. \\
& +16 \mathrm{H}(1,0 ; z)+16 \mathrm{H}(1,1 ; z))+z\left(\frac{11}{2}-2 \pi^{2}+\frac{19}{2} \mathrm{H}(0 ; z)+7 \mathrm{H}(1 ; z)+11 \mathrm{H}(0,0 ; z)\right. \\
& +10 \mathrm{H}(0,1 ; z)+8 \mathrm{H}(1,0 ; z)+8 \mathrm{H}(1,1 ; z))] \\
& +\left[-184+\frac{32 \pi^{2}}{3}+\frac{140 \zeta_{3}}{3}-\frac{4}{3} \pi^{2} \mathrm{H}(-1 ; z)-\frac{185}{2} \mathrm{H}(0 ; z)+9 \pi^{2} \mathrm{H}(0 ; z)-91 \mathrm{H}(1 ; z)\right. \\
& +8 \pi^{2} \mathrm{H}(1 ; z)+8 \mathrm{H}(-1,0 ; z)-38 \mathrm{H}(0,0 ; z)-44 \mathrm{H}(0,1 ; z)-44 \mathrm{H}(1,0 ; z) \\
& -48 \mathrm{H}(1,1 ; z)-16 \mathrm{H}(-1,-1,0 ; z)+8 \mathrm{H}(-1,0,0 ; z)+16 \mathrm{H}(0,-1,0 ; z)-46 \mathrm{H}(0,0,0 ; z) \\
& -44 \mathrm{H}(0,0,1 ; z)-40 \mathrm{H}(0,1,0 ; z)-40 \mathrm{H}(0,1,1 ; z)-16 \mathrm{H}(1,0,0 ; z)-32 \mathrm{H}(1,0,1 ; z) \\
& -24 \mathrm{H}(1,1,0 ; z)-32 \mathrm{H}(1,1,1 ; z)+\frac{1}{z}\left(\frac{485}{2}-\frac{40 \pi^{2}}{3}-\frac{80 \zeta_{3}}{3}-\frac{4}{3} \pi^{2} \mathrm{H}(-1 ; z)+134 \mathrm{H}(0 ; z)\right. \\
& -\frac{20}{3} \pi^{2} \mathrm{H}(0 ; z)+134 \mathrm{H}(1 ; z)-8 \pi^{2} \mathrm{H}(1 ; z)+64 \mathrm{H}(0,0 ; z)+64 \mathrm{H}(0,1 ; z)+64 \mathrm{H}(1,0 ; z) \\
& +64 \mathrm{H}(1,1 ; z)-16 \mathrm{H}(-1,-1,0 ; z)+8 \mathrm{H}(-1,0,0 ; z)+32 \mathrm{H}(0,0,0 ; z)+32 \mathrm{H}(0,0,1 ; z)
\end{aligned}
$$




$$
\begin{aligned}
& +32 \mathrm{H}(0,1,0 ; z)+32 \mathrm{H}(0,1,1 ; z)+16 \mathrm{H}(1,0,0 ; z)+32 \mathrm{H}(1,0,1 ; z)+24 \mathrm{H}(1,1,0 ; z) \\
& +32 \mathrm{H}(1,1,1 ; z))+z\left(\frac{97}{4}-\frac{7 \pi^{2}}{4}-\frac{46 \zeta_{3}}{3}-\frac{2}{3} \pi^{2} \mathrm{H}(-1 ; z)+11 \mathrm{H}(0 ; z)-\frac{9}{2} \pi^{2} \mathrm{H}(0 ; z)\right. \\
& +11 \mathrm{H}(1 ; z)-4 \pi^{2} \mathrm{H}(1 ; z)+8 \mathrm{H}(-1,0 ; z)+\frac{31}{2} \mathrm{H}(0,0 ; z)+19 \mathrm{H}(0,1 ; z)+16 \mathrm{H}(1,0 ; z) \\
& +14 \mathrm{H}(1,1 ; z)-8 \mathrm{H}(-1,-1,0 ; z)+4 \mathrm{H}(-1,0,0 ; z)+23 \mathrm{H}(0,0,0 ; z)+22 \mathrm{H}(0,0,1 ; z) \\
& +20 \mathrm{H}(0,1,0 ; z)+20 \mathrm{H}(0,1,1 ; z)+8 \mathrm{H}(1,0,0 ; z)+16 \mathrm{H}(1,0,1 ; z)+12 \mathrm{H}(1,1,0 ; z) \\
& +16 \mathrm{H}(1,1,1 ; z))] \\
& +\mathcal{O}(\epsilon)
\end{aligned}
$$

The four-quark antenna function contains two different quark flavours. Since it is symmetric under the interchange of the quark-antiquark pairs, we have to consider only one crossing, resulting in $\left(q, q q^{\prime} \bar{q}^{\prime}\right)$, which integrates to

$$
\begin{aligned}
& \mathcal{H}_{q, q q^{\prime} \bar{q}^{\prime}}^{0}= \\
&+\frac{1}{\epsilon^{2}}\left[\frac{4}{3}-\frac{4}{3 z}-\frac{2 z}{3}\right] \\
&+\frac{1}{\epsilon}\left[\frac{50}{9}+\frac{8}{3} \mathrm{H}(0 ; z)+\frac{8}{3} \mathrm{H}(1 ; z)+\frac{1}{z}\left(-\frac{50}{9}-\frac{8}{3} \mathrm{H}(0 ; z)-\frac{8}{3} \mathrm{H}(1 ; z)\right)+z\left(-\frac{19}{9}-\frac{4}{3} \mathrm{H}(0 ; z)\right.\right. \\
&\left.\left.\quad-\frac{4}{3} \mathrm{H}(1 ; z)\right)\right] \\
&+\left[\frac{505}{27}-\frac{10 \pi^{2}}{9}+\frac{100}{9} \mathrm{H}(0 ; z)+\frac{100}{9} \mathrm{H}(1 ; z)+\frac{16}{3} \mathrm{H}(0,0 ; z)+\frac{16}{3} \mathrm{H}(0,1 ; z)+\frac{16}{3} \mathrm{H}(1,0 ; z)\right. \\
& \quad+\frac{16}{3} \mathrm{H}(1,1 ; z)+\frac{1}{z}\left(-\frac{505}{27}+\frac{10 \pi^{2}}{9}-\frac{100}{9} \mathrm{H}(0 ; z)-\frac{100}{9} \mathrm{H}(1 ; z)-\frac{16}{3} \mathrm{H}(0,0 ; z)\right. \\
&\left.\quad-\frac{16}{3} \mathrm{H}(0,1 ; z)-\frac{16}{3} \mathrm{H}(1,0 ; z)-\frac{16}{3} \mathrm{H}(1,1 ; z)\right)+z\left(-\frac{355}{54}+\frac{5 \pi^{2}}{9}\right. \\
&\left.\left.\quad-\frac{38}{9} \mathrm{H}(0 ; z)-\frac{38}{9} \mathrm{H}(1 ; z)-\frac{8}{3} \mathrm{H}(0,0 ; z)-\frac{8}{3} \mathrm{H}(0,1 ; z)-\frac{8}{3} \mathrm{H}(1,0 ; z)-\frac{8}{3} \mathrm{H}(1,1 ; z)\right)\right] \\
&+ \mathcal{O}(\epsilon) .
\end{aligned}
$$

The quark-initiated one-loop virtual gluon-gluon antenna of the form $(q, q g)$ have a leading and subleading colour contribution and a quark loop piece. Their integrals are:

$$
\begin{aligned}
& \mathcal{G}_{q, q g}^{1}= \\
& +\frac{1}{\epsilon^{2}}\left[\frac{3}{4}+\mathrm{H}(0 ; z)+\frac{1}{2} \mathrm{H}(1 ; z)+\frac{1}{z}\left(-\frac{3}{4}-\mathrm{H}(0 ; z)-\frac{1}{2} \mathrm{H}(1 ; z)\right)-z\left(\frac{3}{8}+\frac{1}{2} \mathrm{H}(0 ; z)+\frac{1}{4} \mathrm{H}(1 ; z)\right)\right] \\
& +\frac{1}{\epsilon}\left[-\frac{53}{36}-\frac{\pi^{2}}{3}+\frac{2}{3} \mathrm{H}(0 ; z)+\frac{5}{4} \mathrm{H}(1 ; z)+4 \mathrm{H}(0,0 ; z)+2 \mathrm{H}(0,1 ; z)+2 \mathrm{H}(1,0 ; z)+\frac{3}{2} \mathrm{H}(1,1 ; z)\right. \\
& \quad+\frac{1}{z}\left(\frac{79}{72}+\frac{\pi^{2}}{3}-\frac{13}{6} \mathrm{H}(0 ; z)-2 \mathrm{H}(1 ; z)-4 \mathrm{H}(0,0 ; z)-2 \mathrm{H}(0,1 ; z)-2 \mathrm{H}(1,0 ; z)\right. \\
& \left.\quad-\frac{3}{2} \mathrm{H}(1,1 ; z)\right)+z\left(\frac{10}{9}+\frac{\pi^{2}}{6}+\frac{1}{6} \mathrm{H}(0 ; z)-\frac{3}{8} \mathrm{H}(1 ; z)-2 \mathrm{H}(0,0 ; z)-\mathrm{H}(0,1 ; z)\right.
\end{aligned}
$$




$$
\begin{aligned}
& \left.\left.-\mathrm{H}(1,0 ; z)-\frac{3}{4} \mathrm{H}(1,1 ; z)\right)\right] \\
& +\left[-\frac{571}{108}-\frac{35 \pi^{2}}{12}-5 \zeta_{3}-\frac{73}{36} \mathrm{H}(0 ; z)-\frac{11}{6} \pi^{2} \mathrm{H}(0 ; z)+\frac{1}{36} \mathrm{H}(1 ; z)-\frac{3}{4} \pi^{2} \mathrm{H}(1 ; z)-\mathrm{H}(0,0 ; z)\right. \\
& +\frac{5}{3} \mathrm{H}(0,1 ; z)-\frac{5}{6} \mathrm{H}(1,0 ; z)+\frac{9}{4} \mathrm{H}(1,1 ; z)+10 \mathrm{H}(0,0,0 ; z)+6 \mathrm{H}(0,0,1 ; z)+4 \mathrm{H}(0,1,0 ; z) \\
& +4 \mathrm{H}(0,1,1 ; z)+5 \mathrm{H}(1,0,0 ; z)+4 \mathrm{H}(1,0,1 ; z)+4 \mathrm{H}(1,1,0 ; z)+\frac{7}{2} \mathrm{H}(1,1,1 ; z) \\
& +\frac{1}{z}\left(\frac{3733}{432}+\frac{37 \pi^{2}}{12}+5 \zeta_{3}-\frac{43}{72} \mathrm{H}(0 ; z)+\frac{11}{6} \pi^{2} \mathrm{H}(0 ; z)-\frac{25}{9} \mathrm{H}(1 ; z)+\frac{3}{4} \pi^{2} \mathrm{H}(1 ; z)\right. \\
& -6 \mathrm{H}(0,0 ; z)-\frac{14}{3} \mathrm{H}(0,1 ; z)-\frac{19}{6} \mathrm{H}(1,0 ; z)-\frac{9}{2} \mathrm{H}(1,1 ; z)-10 \mathrm{H}(0,0,0 ; z) \\
& -6 \mathrm{H}(0,0,1 ; z)-4 \mathrm{H}(0,1,0 ; z)-4 \mathrm{H}(0,1,1 ; z)-5 \mathrm{H}(1,0,0 ; z)-4 \mathrm{H}(1,0,1 ; z) \\
& \left.-4 \mathrm{H}(1,1,0 ; z)-\frac{7}{2} \mathrm{H}(1,1,1 ; z)\right)+z\left(\frac{121}{54}+\frac{23 \pi^{2}}{24}+\frac{5 \zeta_{3}}{2}+\frac{20}{9} \mathrm{H}(0 ; z)+\frac{11}{12} \pi^{2} \mathrm{H}(0 ; z)\right. \\
& +\frac{10}{9} \mathrm{H}(1 ; z)+\frac{3}{8} \pi^{2} \mathrm{H}(1 ; z)+\frac{3}{2} \mathrm{H}(0,0 ; z)+\frac{1}{6} \mathrm{H}(0,1 ; z)+\frac{5}{12} \mathrm{H}(1,0 ; z)-\frac{3}{8} \mathrm{H}(1,1 ; z) \\
& -5 \mathrm{H}(0,0,0 ; z)-3 \mathrm{H}(0,0,1 ; z)-2 \mathrm{H}(0,1,0 ; z)-2 \mathrm{H}(0,1,1 ; z)-\frac{5}{2} \mathrm{H}(1,0,0 ; z) \\
& \left.\left.-2 \mathrm{H}(1,0,1 ; z)-2 \mathrm{H}(1,1,0 ; z)-\frac{7}{4} \mathrm{H}(1,1,1 ; z)\right)\right] \\
& +\mathcal{O}(\epsilon) \\
& \tilde{\mathcal{G}}_{q, q g}^{1}= \\
& +\frac{1}{\epsilon^{3}}\left[\frac{1}{2}-\frac{1}{2 z}-\frac{z}{4}\right] \\
& +\frac{1}{\epsilon^{2}}\left[\frac{5}{4}+\mathrm{H}(0 ; z)+\frac{1}{2} \mathrm{H}(1 ; z)-\frac{1}{z}\left(2+\mathrm{H}(0 ; z)+\frac{1}{2} \mathrm{H}(1 ; z)\right)-z\left(\frac{3}{8}+\frac{1}{2} \mathrm{H}(0 ; z)+\frac{1}{4} \mathrm{H}(1 ; z)\right)\right] \\
& +\frac{1}{\epsilon}\left[\frac{17}{4}-\frac{\pi^{2}}{4}+\frac{5}{2} \mathrm{H}(0 ; z)+\frac{5}{4} \mathrm{H}(1 ; z)+2 \mathrm{H}(0,0 ; z)+\mathrm{H}(0,1 ; z)+\mathrm{H}(1,0 ; z)+\frac{1}{2} \mathrm{H}(1,1 ; z)\right. \\
& +\frac{1}{z}\left(-\frac{31}{4}+\frac{\pi^{2}}{4}-4 \mathrm{H}(0 ; z)-2 \mathrm{H}(1 ; z)-2 \mathrm{H}(0,0 ; z)-\mathrm{H}(0,1 ; z)-\mathrm{H}(1,0 ; z)\right. \\
& \left.-\frac{1}{2} \mathrm{H}(1,1 ; z)\right)+z\left(-1+\frac{\pi^{2}}{8}-\frac{3}{4} \mathrm{H}(0 ; z)-\frac{3}{8} \mathrm{H}(1 ; z)-\mathrm{H}(0,0 ; z)-\frac{1}{2} \mathrm{H}(0,1 ; z)\right. \\
& \left.\left.-\frac{1}{2} \mathrm{H}(1,0 ; z)-\frac{1}{4} \mathrm{H}(1,1 ; z)\right)\right] \\
& +\left[\frac{51}{4}-\frac{19 \pi^{2}}{24}-\frac{10 \zeta_{3}}{3}+\frac{35}{4} \mathrm{H}(0 ; z)-\frac{1}{2} \pi^{2} \mathrm{H}(0 ; z)+\frac{17}{4} \mathrm{H}(1 ; z)-\frac{7}{12} \pi^{2} \mathrm{H}(1 ; z)+\frac{9}{2} \mathrm{H}(0,0 ; z)\right. \\
& +\frac{5}{2} \mathrm{H}(0,1 ; z)+2 \mathrm{H}(1,0 ; z)+\frac{5}{4} \mathrm{H}(1,1 ; z)+4 \mathrm{H}(0,0,0 ; z)+2 \mathrm{H}(0,0,1 ; z)+2 \mathrm{H}(0,1,0 ; z) \\
& +\mathrm{H}(0,1,1 ; z)+\mathrm{H}(1,0,0 ; z)+\mathrm{H}(1,0,1 ; z)+\frac{1}{2} \mathrm{H}(1,1,1 ; z)+\frac{1}{z}\left(-\frac{415}{16}+\pi^{2}+\frac{10 \zeta_{3}}{3}\right. \\
& -\frac{31}{2} \mathrm{H}(0 ; z)+\frac{1}{2} \pi^{2} \mathrm{H}(0 ; z)-\frac{67}{8} \mathrm{H}(1 ; z)+\frac{7}{12} \pi^{2} \mathrm{H}(1 ; z)-8 \mathrm{H}(0,0 ; z)-4 \mathrm{H}(0,1 ; z) \\
& -4 \mathrm{H}(1,0 ; z)-2 \mathrm{H}(1,1 ; z)-4 \mathrm{H}(0,0,0 ; z)-2 \mathrm{H}(0,0,1 ; z)-2 \mathrm{H}(0,1,0 ; z)-\mathrm{H}(0,1,1 ; z) \\
& \left.-\mathrm{H}(1,0,0 ; z)-\mathrm{H}(1,0,1 ; z)-\frac{1}{2} \mathrm{H}(1,1,1 ; z)\right)+z\left(-2+\frac{5 \pi^{2}}{48}+\frac{5 \zeta_{3}}{3}-2 \mathrm{H}(0 ; z)\right.
\end{aligned}
$$




$$
\begin{aligned}
+ & \frac{1}{4} \pi^{2} \mathrm{H}(0 ; z)-\mathrm{H}(1 ; z)+\frac{7}{24} \pi^{2} \mathrm{H}(1 ; z)-\frac{7}{4} \mathrm{H}(0,0 ; z)-\frac{3}{4} \mathrm{H}(0,1 ; z)-\mathrm{H}(1,0 ; z) \\
& -\frac{3}{8} \mathrm{H}(1,1 ; z)-2 \mathrm{H}(0,0,0 ; z)-\mathrm{H}(0,0,1 ; z)-\mathrm{H}(0,1,0 ; z)-\frac{1}{2} \mathrm{H}(0,1,1 ; z) \\
& \left.\left.-\frac{1}{2} \mathrm{H}(1,0,0 ; z)-\frac{1}{2} \mathrm{H}(1,0,1 ; z)-\frac{1}{4} \mathrm{H}(1,1,1 ; z)\right)\right] \\
+ & \mathcal{O}(\epsilon) \\
& \hat{\mathcal{G}}_{q, q g}^{1}= \\
+ & \frac{1}{\epsilon}\left[\frac{5}{9}+\frac{1}{3} \mathrm{H}(0 ; z)-\frac{1}{z}\left(\frac{29}{36}+\frac{1}{3} \mathrm{H}(0 ; z)\right)-z\left(\frac{5}{18}+\frac{1}{6} \mathrm{H}(0 ; z)\right)\right] \\
+ & {\left[\frac{52}{27}+\frac{\pi^{2}}{4}+\frac{13}{9} \mathrm{H}(0 ; z)+\frac{5}{9} \mathrm{H}(1 ; z)+\mathrm{H}(0,0 ; z)+\frac{1}{3} \mathrm{H}(0,1 ; z)+\frac{1}{3} \mathrm{H}(1,0 ; z)+\frac{1}{z}\left(-\frac{415}{108}\right.\right.} \\
& \left.-\frac{\pi^{2}}{4}-\frac{79}{36} \mathrm{H}(0 ; z)-\frac{29}{36} \mathrm{H}(1 ; z)-\mathrm{H}(0,0 ; z)-\frac{1}{3} \mathrm{H}(0,1 ; z)-\frac{1}{3} \mathrm{H}(1,0 ; z)\right)+z\left(-\frac{14}{27}\right. \\
& \left.\left.-\frac{\pi^{2}}{8}-\frac{5}{9} \mathrm{H}(0 ; z)-\frac{5}{18} \mathrm{H}(1 ; z)-\frac{1}{2} \mathrm{H}(0,0 ; z)-\frac{1}{6} \mathrm{H}(0,1 ; z)-\frac{1}{6} \mathrm{H}(1,0 ; z)\right)\right] \\
& +\mathcal{O}(\epsilon) .
\end{aligned}
$$

\section{Gluon initiated antennae}

The gluon-initiated initial-final antenna functions are obtained from final-final antenna functions listed in [65] by crossing a gluon into the initial state. As in the quark case, their unintegrated forms are pure kinematical crossings of the final-final expressions, with no symmetry factors or polarization sums multiplied on them. Also, we do not provide a decomposition into sub-antennae here. The precise decomposition depends on the requirements and symmetries of the process under consideration, and is normally performed in the context of an actual calculation.

As in the quark-initiated case, some attention has to be paid in the crossing of the virtual one-loop antenna functions from the final-final kinematics [65] to the initial-final kinematics. This crossing requires the analytical continuation of the polylogarithmic functions to the relevant phase space, again consisting of three different Riemann sheets [119-126].

All gluon-initiated initial-final antenna functions are listed in table 2, their integrated forms are collected in the following.

\subsection{Quark-quark antennae}

The NLO quark-antiquark antenna function to order $\epsilon^{2}$ is:

$$
\begin{aligned}
& \mathcal{A}_{g, q \bar{q}}^{0}= \\
+ & \frac{1}{\epsilon}\left[1-2 z+2 z^{2}\right] \\
+ & {\left[\left(1-2 z+2 z^{2}\right)(\mathrm{H}(0 ; z)+\mathrm{H}(1 ; z))\right] }
\end{aligned}
$$




$$
\begin{aligned}
& +\epsilon\left[2+\left(1-2 z+2 z^{2}\right)\left(-\frac{\pi^{2}}{4}+\mathrm{H}(0,0 ; z)+\mathrm{H}(0,1 ; z)+\mathrm{H}(1,0 ; z)+\mathrm{H}(1,1 ; z)\right)\right] \\
& +\epsilon^{2}\left[4+2 \mathrm{H}(0 ; z)+2 \mathrm{H}(1 ; z)+\left(1-2 z+2 z^{2}\right)\left(-\frac{7 \zeta_{3}}{3}-\frac{1}{4} \pi^{2} \mathrm{H}(0 ; z)-\frac{1}{4} \pi^{2} \mathrm{H}(1 ; z)\right.\right. \\
& \quad+\mathrm{H}(0,0,0 ; z)+\mathrm{H}(0,0,1 ; z)+\mathrm{H}(0,1,0 ; z)+\mathrm{H}(0,1,1 ; z)+\mathrm{H}(1,0,0 ; z)+\mathrm{H}(1,0,1 ; z) \\
& \quad+\mathrm{H}(1,1,0 ; z)+\mathrm{H}(1,1,1 ; z))] \\
& \quad+\mathcal{O}\left(\epsilon^{3}\right) .
\end{aligned}
$$

For the NNLO double real radiation antenna functions, we have the leading and subleading colour contributions to $(g, g q \bar{q})$, whose integrals read

$$
\begin{aligned}
& \mathcal{A}_{g, g q \bar{q}}^{0}= \\
& +\frac{1}{\epsilon^{3}}\left[3-6 z+6 z^{2}\right] \\
& +\frac{1}{\epsilon^{2}}\left[\frac{13}{4}-\frac{4}{3 z}+\frac{7}{2} \mathrm{H}(0 ; z)+6 \mathrm{H}(1 ; z)+z(-15-19 \mathrm{H}(0 ; z)-12 \mathrm{H}(1 ; z))\right. \\
& \left.+z^{2}\left(\frac{49}{3}+12 \mathrm{H}(0 ; z)+12 \mathrm{H}(1 ; z)\right)\right] \\
& +\frac{1}{\epsilon}\left[\frac{173}{12}-2 \pi^{2}+\frac{27}{4} \mathrm{H}(0 ; z)+\frac{13}{2} \mathrm{H}(1 ; z)-2 \mathrm{H}(-1,0 ; z)+\frac{11}{2} \mathrm{H}(0,0 ; z)+7 \mathrm{H}(0,1 ; z)\right. \\
& +11 \mathrm{H}(1,0 ; z)+12 \mathrm{H}(1,1 ; z)+\frac{1}{z}\left(\frac{8}{9}-\frac{8}{3} \mathrm{H}(1 ; z)\right)+z\left(-\frac{89}{12}+\frac{22 \pi^{2}}{3}-16 \mathrm{H}(0 ; z)\right. \\
& -30 \mathrm{H}(1 ; z)-4 \mathrm{H}(-1,0 ; z)-43 \mathrm{H}(0,0 ; z)-38 \mathrm{H}(0,1 ; z)-22 \mathrm{H}(1,0 ; z)-24 \mathrm{H}(1,1 ; z)) \\
& +z^{2}\left(\frac{91}{9}-\frac{17 \pi^{2}}{3}+\frac{142}{3} \mathrm{H}(0 ; z)+\frac{98}{3} \mathrm{H}(1 ; z)-4 \mathrm{H}(-1,0 ; z)+26 \mathrm{H}(0,0 ; z)+24 \mathrm{H}(0,1 ; z)\right. \\
& +22 \mathrm{H}(1,0 ; z)+24 \mathrm{H}(1,1 ; z))] \\
& +\left[\frac{1895}{36}-\frac{67 \pi^{2}}{24}-41 \zeta_{3}+\pi^{2} \mathrm{H}(-1 ; z)+\frac{9}{4} \mathrm{H}(0 ; z)-\frac{15}{4} \pi^{2} \mathrm{H}(0 ; z)+\frac{173}{6} \mathrm{H}(1 ; z)-5 \pi^{2} \mathrm{H}(1 ; z)\right. \\
& +2 \mathrm{H}(-1,0 ; z)+\frac{63}{4} \mathrm{H}(0,0 ; z)+\frac{27}{2} \mathrm{H}(0,1 ; z)+13 \mathrm{H}(1,0 ; z)+13 \mathrm{H}(1,1 ; z) \\
& +4 \mathrm{H}(-1,-1,0 ; z)-8 \mathrm{H}(-1,0,0 ; z)-4 \mathrm{H}(-1,0,1 ; z)-8 \mathrm{H}(0,-1,0 ; z)+\frac{19}{2} \mathrm{H}(0,0,0 ; z) \\
& +11 \mathrm{H}(0,0,1 ; z)+10 \mathrm{H}(0,1,0 ; z)+14 \mathrm{H}(0,1,1 ; z)+17 \mathrm{H}(1,0,0 ; z)+22 \mathrm{H}(1,0,1 ; z) \\
& +22 \mathrm{H}(1,1,0 ; z)+24 \mathrm{H}(1,1,1 ; z)+\frac{2}{3 z^{2}} \mathrm{H}(-1,0 ; z)+\frac{1}{z}\left(-\frac{502}{27}+\frac{2 \pi^{2}}{3}-\frac{2}{3} \mathrm{H}(0 ; z)\right. \\
& \left.+\frac{16}{9} \mathrm{H}(1 ; z)+\frac{8}{3} \mathrm{H}(-1,0 ; z)-\frac{16}{3} \mathrm{H}(1,0 ; z)-\frac{16}{3} \mathrm{H}(1,1 ; z)\right)+z\left(-\frac{1135}{18}+\frac{185 \pi^{2}}{18}+86 \zeta_{3}\right. \\
& +2 \pi^{2} \mathrm{H}(-1 ; z)-\frac{217}{4} \mathrm{H}(0 ; z)+\frac{121}{6} \pi^{2} \mathrm{H}(0 ; z)-\frac{89}{6} \mathrm{H}(1 ; z)+10 \pi^{2} \mathrm{H}(1 ; z) \\
& +\frac{56}{3} \mathrm{H}(-1,0 ; z)-\frac{116}{3} \mathrm{H}(0,0 ; z)-32 \mathrm{H}(0,1 ; z)-62 \mathrm{H}(1,0 ; z)-60 \mathrm{H}(1,1 ; z) \\
& +8 \mathrm{H}(-1,-1,0 ; z)-16 \mathrm{H}(-1,0,0 ; z)-8 \mathrm{H}(-1,0,1 ; z)-91 \mathrm{H}(0,0,0 ; z)-86 \mathrm{H}(0,0,1 ; z) \\
& -68 \mathrm{H}(0,1,0 ; z)-76 \mathrm{H}(0,1,1 ; z)-34 \mathrm{H}(1,0,0 ; z)-44 \mathrm{H}(1,0,1 ; z)-44 \mathrm{H}(1,1,0 ; z)
\end{aligned}
$$




$$
\begin{aligned}
& -48 \mathrm{H}(1,1,1 ; z))+z^{2}\left(\frac{2329}{27}-\frac{37 \pi^{2}}{2}-92 \zeta_{3}+2 \pi^{2} \mathrm{H}(-1 ; z)-\frac{86}{9} \mathrm{H}(0 ; z)-\frac{40}{3} \pi^{2} \mathrm{H}(0 ; z)\right. \\
& +\frac{182}{9} \mathrm{H}(1 ; z)-10 \pi^{2} \mathrm{H}(1 ; z)+\frac{44}{3} \mathrm{H}(-1,0 ; z)+\frac{328}{3} \mathrm{H}(0,0 ; z)+\frac{284}{3} \mathrm{H}(0,1 ; z) \\
& +\frac{196}{3} \mathrm{H}(1,0 ; z)+\frac{196}{3} \mathrm{H}(1,1 ; z)+8 \mathrm{H}(-1,-1,0 ; z)-16 \mathrm{H}(-1,0,0 ; z)-8 \mathrm{H}(-1,0,1 ; z) \\
& -16 \mathrm{H}(0,-1,0 ; z)+54 \mathrm{H}(0,0,0 ; z)+52 \mathrm{H}(0,0,1 ; z)+40 \mathrm{H}(0,1,0 ; z)+48 \mathrm{H}(0,1,1 ; z) \\
& +34 \mathrm{H}(1,0,0 ; z)+44 \mathrm{H}(1,0,1 ; z)+44 \mathrm{H}(1,1,0 ; z)+48 \mathrm{H}(1,1,1 ; z))] \\
& +\mathcal{O}(\epsilon)
\end{aligned}
$$

and

$$
\begin{aligned}
& \tilde{\mathcal{A}}_{g, g q \bar{q}}^{0}= \\
& +\frac{1}{\epsilon^{3}}\left[4-8 z+8 z^{2}\right] \\
& +\frac{1}{\epsilon^{2}}\left[\frac{13}{2}+7 \mathrm{H}(0 ; z)+8 \mathrm{H}(1 ; z)-z(14+14 \mathrm{H}(0 ; z)+16 \mathrm{H}(1 ; z))\right. \\
& \left.+z^{2}(12+16 \mathrm{H}(0 ; z)+16 \mathrm{H}(1 ; z))\right] \\
& +\frac{1}{\epsilon}\left[\frac{39}{2}-\frac{7 \pi^{2}}{3}+\frac{15}{2} \mathrm{H}(0 ; z)+13 \mathrm{H}(1 ; z)+15 \mathrm{H}(0,0 ; z)+14 \mathrm{H}(0,1 ; z)+18 \mathrm{H}(1,0 ; z)\right. \\
& +16 \mathrm{H}(1,1 ; z)+z\left(-\frac{75}{2}+\frac{14 \pi^{2}}{3}-32 \mathrm{H}(0 ; z)-28 \mathrm{H}(1 ; z)-30 \mathrm{H}(0,0 ; z)-28 \mathrm{H}(0,1 ; z)\right. \\
& -36 \mathrm{H}(1,0 ; z)-32 \mathrm{H}(1,1 ; z))+z^{2}\left(38-\frac{16 \pi^{2}}{3}+24 \mathrm{H}(0 ; z)+24 \mathrm{H}(1 ; z)+36 \mathrm{H}(0,0 ; z)\right. \\
& +32 \mathrm{H}(0,1 ; z)+36 \mathrm{H}(1,0 ; z)+32 \mathrm{H}(1,1 ; z))] \\
& +\left[\frac{301}{6}-\frac{9 \pi^{2}}{4}-\frac{86 \zeta_{3}}{3}+\frac{4}{3} \pi^{2} \mathrm{H}(-1 ; z)+\frac{199}{6} \mathrm{H}(0 ; z)-\frac{37}{6} \pi^{2} \mathrm{H}(0 ; z)+39 \mathrm{H}(1 ; z)-\frac{22}{3} \pi^{2} \mathrm{H}(1 ; z)\right. \\
& -24 \mathrm{H}(-1,0 ; z)+\frac{27}{2} \mathrm{H}(0,0 ; z)+15 \mathrm{H}(0,1 ; z)+30 \mathrm{H}(1,0 ; z)+26 \mathrm{H}(1,1 ; z) \\
& +16 \mathrm{H}(-1,-1,0 ; z)-8 \mathrm{H}(-1,0,0 ; z)-16 \mathrm{H}(0,-1,0 ; z)+31 \mathrm{H}(0,0,0 ; z)+30 \mathrm{H}(0,0,1 ; z) \\
& +28 \mathrm{H}(0,1,0 ; z)+28 \mathrm{H}(0,1,1 ; z)+26 \mathrm{H}(1,0,0 ; z)+36 \mathrm{H}(1,0,1 ; z)+28 \mathrm{H}(1,1,0 ; z) \\
& +32 \mathrm{H}(1,1,1 ; z)+\frac{4}{3 z^{2}} \mathrm{H}(-1,0 ; z)+\frac{1}{z}\left(\frac{4}{3}-\frac{4}{3} \mathrm{H}(0 ; z)\right)+z\left(-99+\frac{77 \pi^{2}}{9}+\frac{76 \zeta_{3}}{3}\right. \\
& +\frac{8}{3} \pi^{2} \mathrm{H}(-1 ; z)-\frac{153}{2} \mathrm{H}(0 ; z)+\frac{37}{3} \pi^{2} \mathrm{H}(0 ; z)-75 \mathrm{H}(1 ; z)+\frac{44}{3} \pi^{2} \mathrm{H}(1 ; z)-\frac{56}{3} \mathrm{H}(-1,0 ; z) \\
& -\frac{160}{3} \mathrm{H}(0,0 ; z)-64 \mathrm{H}(0,1 ; z)-60 \mathrm{H}(1,0 ; z)-56 \mathrm{H}(1,1 ; z)+32 \mathrm{H}(-1,-1,0 ; z) \\
& -16 \mathrm{H}(-1,0,0 ; z)-62 \mathrm{H}(0,0,0 ; z)-60 \mathrm{H}(0,0,1 ; z)-56 \mathrm{H}(0,1,0 ; z)-56 \mathrm{H}(0,1,1 ; z) \\
& -52 \mathrm{H}(1,0,0 ; z)-72 \mathrm{H}(1,0,1 ; z)-56 \mathrm{H}(1,1,0 ; z)-64 \mathrm{H}(1,1,1 ; z))+z^{2}\left(98-10 \pi^{2}\right. \\
& -\frac{208 \zeta_{3}}{3}+\frac{4}{3} \pi^{2} \mathrm{H}(-1 ; z)+76 \mathrm{H}(0 ; z)-\frac{44}{3} \pi^{2} \mathrm{H}(0 ; z)+76 \mathrm{H}(1 ; z)-\frac{40}{3} \pi^{2} \mathrm{H}(1 ; z) \\
& +48 \mathrm{H}(0,0 ; z)+48 \mathrm{H}(0,1 ; z)+48 \mathrm{H}(1,0 ; z)+48 \mathrm{H}(1,1 ; z)+16 \mathrm{H}(-1,-1,0 ; z) \\
& -8 \mathrm{H}(-1,0,0 ; z)-16 \mathrm{H}(0,-1,0 ; z)+76 \mathrm{H}(0,0,0 ; z)+72 \mathrm{H}(0,0,1 ; z)+64 \mathrm{H}(0,1,0 ; z)
\end{aligned}
$$




$$
\begin{aligned}
& \quad+64 \mathrm{H}(0,1,1 ; z)+68 \mathrm{H}(1,0,0 ; z)+72 \mathrm{H}(1,0,1 ; z)+64 \mathrm{H}(1,1,0 ; z)+64 \mathrm{H}(1,1,1 ; z))] \\
& +\mathcal{O}(\epsilon) .
\end{aligned}
$$

The one-loop corrections to the $(q, q \bar{q})$ antenna function have a leading and subleading colour term as well as a closed quark loop contribution. These integrate to

$$
\begin{aligned}
& \mathcal{A}_{g, q \bar{q}}^{1}= \\
& +\frac{1}{\epsilon^{3}}\left[-\frac{1}{2}+z-z^{2}\right] \\
& +\frac{1}{\epsilon^{2}}\left[-\frac{7}{3}-\frac{3}{2} \mathrm{H}(0 ; z)-\frac{1}{2} \mathrm{H}(1 ; z)+z\left(\frac{11}{3}+3 \mathrm{H}(0 ; z)+\mathrm{H}(1 ; z)\right)\right. \\
& \left.+z^{2}\left(-\frac{11}{3}-3 \mathrm{H}(0 ; z)-\mathrm{H}(1 ; z)\right)\right] \\
& +\frac{1}{\epsilon}\left[-4+\frac{\pi^{2}}{2}-\frac{10}{3} \mathrm{H}(0 ; z)-\frac{7}{3} \mathrm{H}(1 ; z)-4 \mathrm{H}(0,0 ; z)-\frac{3}{2} \mathrm{H}(0,1 ; z)-\mathrm{H}(1,0 ; z)-\frac{1}{2} \mathrm{H}(1,1 ; z)\right. \\
& +z\left(\frac{1}{4}-\pi^{2}+\frac{11}{3} \mathrm{H}(0 ; z)+\frac{11}{3} \mathrm{H}(1 ; z)+8 \mathrm{H}(0,0 ; z)+3 \mathrm{H}(0,1 ; z)+2 \mathrm{H}(1,0 ; z)+\mathrm{H}(1,1 ; z)\right) \\
& \left.+z^{2}\left(\pi^{2}-\frac{11}{3} \mathrm{H}(0 ; z)-\frac{11}{3} \mathrm{H}(1 ; z)-8 \mathrm{H}(0,0 ; z)-3 \mathrm{H}(0,1 ; z)-2 \mathrm{H}(1,0 ; z)-\mathrm{H}(1,1 ; z)\right)\right] \\
& +\left[-\frac{227}{12}+\frac{19 \pi^{2}}{24}+\frac{53 \zeta_{3}}{6}-11 \mathrm{H}(0 ; z)+\frac{19}{12} \pi^{2} \mathrm{H}(0 ; z)-4 \mathrm{H}(1 ; z)+\frac{7}{12} \pi^{2} \mathrm{H}(1 ; z)-\frac{19}{3} \mathrm{H}(0,0 ; z)\right. \\
& -\frac{10}{3} \mathrm{H}(0,1 ; z)-\frac{10}{3} \mathrm{H}(1,0 ; z)-\frac{7}{3} \mathrm{H}(1,1 ; z)-9 \mathrm{H}(0,0,0 ; z)-4 \mathrm{H}(0,0,1 ; z)-2 \mathrm{H}(0,1,0 ; z) \\
& -\frac{3}{2} \mathrm{H}(0,1,1 ; z)-\mathrm{H}(1,0,0 ; z)-\mathrm{H}(1,0,1 ; z)-\frac{1}{2} \mathrm{H}(1,1,1 ; z)+z\left(\frac{1}{2}-\frac{7 \pi^{2}}{12}-\frac{53 \zeta_{3}}{3}\right. \\
& +\frac{1}{2} \mathrm{H}(0 ; z)-\frac{19}{6} \pi^{2} \mathrm{H}(0 ; z)+\frac{1}{4} \mathrm{H}(1 ; z)-\frac{7}{6} \pi^{2} \mathrm{H}(1 ; z)+\frac{14}{3} \mathrm{H}(0,0 ; z)+\frac{11}{3} \mathrm{H}(0,1 ; z) \\
& +\frac{14}{3} \mathrm{H}(1,0 ; z)+\frac{11}{3} \mathrm{H}(1,1 ; z)+18 \mathrm{H}(0,0,0 ; z)+8 \mathrm{H}(0,0,1 ; z)+4 \mathrm{H}(0,1,0 ; z) \\
& +3 \mathrm{H}(0,1,1 ; z)+2 \mathrm{H}(1,0,0 ; z)+2 \mathrm{H}(1,0,1 ; z)+\mathrm{H}(1,1,1 ; z))+z^{2}\left(\frac{11 \pi^{2}}{12}+\frac{53 \zeta_{3}}{3}\right. \\
& +\frac{19}{6} \pi^{2} \mathrm{H}(0 ; z)+\frac{7}{6} \pi^{2} \mathrm{H}(1 ; z)-\frac{11}{3} \mathrm{H}(0,0 ; z)-\frac{11}{3} \mathrm{H}(0,1 ; z)-\frac{11}{3} \mathrm{H}(1,0 ; z) \\
& -\frac{11}{3} \mathrm{H}(1,1 ; z)-18 \mathrm{H}(0,0,0 ; z)-8 \mathrm{H}(0,0,1 ; z)-4 \mathrm{H}(0,1,0 ; z)-3 \mathrm{H}(0,1,1 ; z) \\
& -2 \mathrm{H}(1,0,0 ; z)-2 \mathrm{H}(1,0,1 ; z)-\mathrm{H}(1,1,1 ; z))] \\
& +\mathcal{O}(\epsilon)
\end{aligned}
$$

and

$$
\begin{aligned}
& \tilde{\mathcal{A}}_{g, q \bar{q}}^{1}= \\
& +\frac{1}{\epsilon^{2}}\left[-\frac{1}{2} \mathrm{H}(0 ; z)-\frac{1}{2} \mathrm{H}(1 ; z)+z(\mathrm{H}(0 ; z)+\mathrm{H}(1 ; z))-z^{2}(\mathrm{H}(0 ; z)+\mathrm{H}(1 ; z))\right] \\
& +\frac{1}{\epsilon}\left[\frac{\pi^{2}}{12}-\frac{1}{2} \mathrm{H}(0 ; z)-\frac{1}{2} \mathrm{H}(1 ; z)-2 \mathrm{H}(0,0 ; z)-\frac{3}{2} \mathrm{H}(0,1 ; z)-2 \mathrm{H}(1,0 ; z)-\frac{3}{2} \mathrm{H}(1,1 ; z)\right. \\
& \quad+z\left(-\frac{1}{4}-\frac{\pi^{2}}{6}+4 \mathrm{H}(0,0 ; z)+3 \mathrm{H}(0,1 ; z)+4 \mathrm{H}(1,0 ; z)+3 \mathrm{H}(1,1 ; z)\right)
\end{aligned}
$$




$$
\begin{aligned}
& \left.+z^{2}\left(\frac{\pi^{2}}{6}-4 \mathrm{H}(0,0 ; z)-3 \mathrm{H}(0,1 ; z)-4 \mathrm{H}(1,0 ; z)-3 \mathrm{H}(1,1 ; z)\right)\right] \\
+\left[\frac{5}{4}\right. & -\frac{\pi^{2}}{12}-\frac{3 \zeta_{3}}{2}-3 \mathrm{H}(0 ; z)+\frac{3}{4} \pi^{2} \mathrm{H}(0 ; z)-4 \mathrm{H}(1 ; z)+\frac{13}{12} \pi^{2} \mathrm{H}(1 ; z)-\frac{5}{2} \mathrm{H}(0,0 ; z) \\
& -\frac{3}{2} \mathrm{H}(0,1 ; z)-\frac{5}{2} \mathrm{H}(1,0 ; z)-\frac{3}{2} \mathrm{H}(1,1 ; z)-5 \mathrm{H}(0,0,0 ; z)-4 \mathrm{H}(0,0,1 ; z) \\
& -4 \mathrm{H}(0,1,0 ; z)-\frac{7}{2} \mathrm{H}(0,1,1 ; z)-4 \mathrm{H}(1,0,0 ; z)-4 \mathrm{H}(1,0,1 ; z)-3 \mathrm{H}(1,1,0 ; z) \\
& -\frac{7}{2} \mathrm{H}(1,1,1 ; z)+z\left(-\frac{3}{2}+\frac{\pi^{2}}{3}+3 \zeta_{3}-\frac{1}{2} \mathrm{H}(0 ; z)-\frac{3}{2} \pi^{2} \mathrm{H}(0 ; z)-\frac{1}{4} \mathrm{H}(1 ; z)-\frac{13}{6} \pi^{2} \mathrm{H}(1 ; z)\right. \\
& +\mathrm{H}(0,0 ; z)+\mathrm{H}(1,0 ; z)+10 \mathrm{H}(0,0,0 ; z)+8 \mathrm{H}(0,0,1 ; z)+8 \mathrm{H}(0,1,0 ; z)+7 \mathrm{H}(0,1,1 ; z) \\
& +8 \mathrm{H}(1,0,0 ; z)+8 \mathrm{H}(1,0,1 ; z)+6 \mathrm{H}(1,1,0 ; z)+7 \mathrm{H}(1,1,1 ; z))+z^{2}\left(-\zeta_{3}+\mathrm{H}(0 ; z)\right. \\
& +\frac{3}{2} \pi^{2} \mathrm{H}(0 ; z)+\mathrm{H}(1 ; z)+\frac{3}{2} \pi^{2} \mathrm{H}(1 ; z)-10 \mathrm{H}(0,0,0 ; z)-8 \mathrm{H}(0,0,1 ; z)-8 \mathrm{H}(0,1,0 ; z) \\
& -7 \mathrm{H}(0,1,1 ; z)-10 \mathrm{H}(1,0,0 ; z)-8 \mathrm{H}(1,0,1 ; z)-8 \mathrm{H}(1,1,0 ; z)-7 \mathrm{H}(1,1,1 ; z))] \\
+ & \mathcal{O}(\epsilon)
\end{aligned}
$$

and

$$
\begin{aligned}
\hat{\mathcal{A}}_{g, q \bar{q}}^{1}= \\
+\frac{1}{\epsilon^{2}}\left[\frac{1}{3}-\frac{2 z}{3}+\frac{2 z^{2}}{3}\right] \\
+\frac{1}{\epsilon}\left[\frac{1}{3} \mathrm{H}(0 ; z)+\frac{1}{3} \mathrm{H}(1 ; z)+z\left(-\frac{2}{3} \mathrm{H}(0 ; z)-\frac{2}{3} \mathrm{H}(1 ; z)\right)+z^{2}\left(\frac{2}{3} \mathrm{H}(0 ; z)+\frac{2}{3} \mathrm{H}(1 ; z)\right)\right] \\
+\left[\frac{2}{3}-\frac{\pi^{2}}{12}+\frac{1}{3} \mathrm{H}(0,0 ; z)+\frac{1}{3} \mathrm{H}(0,1 ; z)+\frac{1}{3} \mathrm{H}(1,0 ; z)+\frac{1}{3} \mathrm{H}(1,1 ; z)+z\left(\frac{\pi^{2}}{6}-\frac{2}{3} \mathrm{H}(0,0 ; z)\right.\right. \\
\left.\quad-\frac{2}{3} \mathrm{H}(0,1 ; z)-\frac{2}{3} \mathrm{H}(1,0 ; z)-\frac{2}{3} \mathrm{H}(1,1 ; z)\right)+z^{2}\left(-\frac{\pi^{2}}{6}+\frac{2}{3} \mathrm{H}(0,0 ; z)+\frac{2}{3} \mathrm{H}(0,1 ; z)\right. \\
\left.\left.\quad+\frac{2}{3} \mathrm{H}(1,0 ; z)+\frac{2}{3} \mathrm{H}(1,1 ; z)\right)\right] \\
+\mathcal{O}(\epsilon) .
\end{aligned}
$$

\subsection{Quark-gluon antennae}

The integrated gluon-initiated $(g, q q)$ antenna function at NLO is through to order $\epsilon^{2}$ :

$$
\begin{aligned}
& \mathcal{D}_{g, g q}^{0}= \\
+ & \frac{1}{\epsilon^{2}}[\delta(1-z)] \\
+ & \frac{1}{\epsilon}\left[\frac{5}{2}+\frac{3}{4} \delta(1-z)-\mathcal{D}_{0}(z)-\frac{1}{z}-2 z+2 z^{2}\right] \\
+ & {\left[-\frac{1}{2}+\delta(1-z)\left(\frac{7}{4}-\frac{\pi^{2}}{4}\right)-\frac{3}{4} \mathcal{D}_{0}(z)+\mathcal{D}_{1}(z)+\frac{5}{2} \mathrm{H}(0 ; z)+\frac{5}{2} \mathrm{H}(1 ; z)-\frac{1}{1-z} \mathrm{H}(0 ; z)\right.} \\
& \left.\quad-\frac{1}{z}\left(\frac{3}{4}+\mathrm{H}(0 ; z)+\mathrm{H}(1 ; z)\right)+\left(-z+z^{2}\right)(2 \mathrm{H}(0 ; z)+2 \mathrm{H}(1 ; z))\right]
\end{aligned}
$$




$$
\begin{aligned}
+\epsilon[ & 1-\frac{5 \pi^{2}}{8}+\delta(1-z)\left(\frac{7}{2}-\frac{3 \pi^{2}}{16}-\frac{7 \zeta_{3}}{3}\right)+\left(-\frac{7}{4}+\frac{\pi^{2}}{4}\right) \mathcal{D}_{0}(z)+\frac{3}{4} \mathcal{D}_{1}(z)-\frac{1}{2} \mathcal{D}_{2}(z)-\frac{1}{2} \mathrm{H}(0 ; z) \\
& -\frac{1}{2} \mathrm{H}(1 ; z)+\frac{5}{2} \mathrm{H}(0,0 ; z)+\frac{5}{2} \mathrm{H}(0,1 ; z)+\frac{5}{2} \mathrm{H}(1,0 ; z)+\frac{5}{2} \mathrm{H}(1,1 ; z)-\frac{1}{1-z}\left(\frac{3}{4} \mathrm{H}(0 ; z)\right. \\
& +\mathrm{H}(0,0 ; z)+\mathrm{H}(0,1 ; z)+\mathrm{H}(1,0 ; z))+\frac{1}{z}\left(-\frac{7}{4}+\frac{\pi^{2}}{4}-\frac{3}{4} \mathrm{H}(0 ; z)-\frac{3}{4} \mathrm{H}(1 ; z)\right. \\
& -\mathrm{H}(0,0 ; z)-\mathrm{H}(0,1 ; z)-\mathrm{H}(1,0 ; z)-\mathrm{H}(1,1 ; z))+\left(z-z^{2}\right)\left(\frac{\pi^{2}}{2}-2 \mathrm{H}(0,0 ; z)\right. \\
& -2 \mathrm{H}(0,1 ; z)-2 \mathrm{H}(1,0 ; z)-2 \mathrm{H}(1,1 ; z))] \\
+\epsilon^{2} & {\left[3+\frac{\pi^{2}}{8}-\frac{35 \zeta_{3}}{6}+\delta(1-z)\left(7-\frac{7 \pi^{2}}{16}-\frac{\pi^{4}}{96}-\frac{7 \zeta_{3}}{4}\right)+\left(-\frac{7}{2}+\frac{3 \pi^{2}}{16}+\frac{7 \zeta_{3}}{3}\right) \mathcal{D}_{0}(z)+\left(\frac{7}{4}-\frac{\pi^{2}}{4}\right) \mathcal{D}_{1}(z)\right.} \\
& -\frac{3}{8} \mathcal{D}_{2}(z)+\frac{1}{6} \mathcal{D}_{3}(z)+\mathrm{H}(0 ; z)-\frac{5}{8} \pi^{2} \mathrm{H}(0 ; z)+\mathrm{H}(1 ; z)-\frac{5}{8} \pi^{2} \mathrm{H}(1 ; z)-\frac{1}{2} \mathrm{H}(0,0 ; z) \\
& -\frac{1}{2} \mathrm{H}(0,1 ; z)-\frac{1}{2} \mathrm{H}(1,0 ; z)-\frac{1}{2} \mathrm{H}(1,1 ; z)+\frac{5}{2} \mathrm{H}(0,0,0 ; z)+\frac{5}{2} \mathrm{H}(0,0,1 ; z)+\frac{5}{2} \mathrm{H}(0,1,0 ; z) \\
& +\frac{5}{2} \mathrm{H}(0,1,1 ; z)+\frac{5}{2} \mathrm{H}(1,0,0 ; z)+\frac{5}{2} \mathrm{H}(1,0,1 ; z)+\frac{5}{2} \mathrm{H}(1,1,0 ; z)+\frac{5}{2} \mathrm{H}(1,1,1 ; z) \\
& +\frac{1}{1-z}\left(-\frac{7}{4} \mathrm{H}(0 ; z)+\frac{1}{4} \pi^{2} \mathrm{H}(0 ; z)-\frac{3}{4} \mathrm{H}(0,0 ; z)-\frac{3}{4} \mathrm{H}(0,1 ; z)-\frac{3}{4} \mathrm{H}(1,0 ; z)\right. \\
& -\mathrm{H}(0,0,0 ; z)-\mathrm{H}(0,0,1 ; z)-\mathrm{H}(0,1,0 ; z)-\mathrm{H}(0,1,1 ; z)-\mathrm{H}(1,0,0 ; z)-\mathrm{H}(1,0,1 ; z) \\
& -\mathrm{H}(1,1,0 ; z))+\frac{1}{z}\left(-\frac{7}{2}+\frac{3 \pi^{2}}{16}+\frac{7 \zeta_{3}}{3}-\frac{7}{4} \mathrm{H}(0 ; z)+\frac{1}{4} \pi^{2} \mathrm{H}(0 ; z)-\frac{7}{4} \mathrm{H}(1 ; z)+\frac{1}{4} \pi^{2} \mathrm{H}(1 ; z)\right. \\
& -\frac{3}{4} \mathrm{H}(0,0 ; z)-\frac{3}{4} \mathrm{H}(0,1 ; z)-\frac{3}{4} \mathrm{H}(1,0 ; z)-\frac{3}{4} \mathrm{H}(1,1 ; z)-\mathrm{H}(0,0,0 ; z)-\mathrm{H}(0,0,1 ; z) \\
& -\mathrm{H}(0,1,0 ; z)-\mathrm{H}(0,1,1 ; z)-\mathrm{H}(1,0,0 ; z)-\mathrm{H}(1,0,1 ; z)-\mathrm{H}(1,1,0 ; z)-\mathrm{H}(1,1,1 ; z)) \\
& +\left(z-z^{2}\right)\left(\frac{14 \zeta_{3}}{3}+\frac{1}{2} \pi^{2} \mathrm{H}(0 ; z)+\frac{1}{2} \pi^{2} \mathrm{H}(1 ; z)-2 \mathrm{H}(0,0,0 ; z)-2 \mathrm{H}(0,0,1 ; z)-2 \mathrm{H}(0,1,0 ; z)\right. \\
& -2 \mathrm{H}(0,1,1 ; z)-2 \mathrm{H}(1,0,0 ; z)-2 \mathrm{H}(1,0,1 ; z)-2 \mathrm{H}(1,1,0 ; z)-2 \mathrm{H}(1,1,1 ; z))] \\
+ & \left(\epsilon^{3}\right) .
\end{aligned}
$$

In crossing one of the gluons in the $D_{4}^{0}$ final-final quark-gluon antenna function into the initial state, one has to distinguish two cases, depending on whether the gluon is colourconnected to the quark or not. If the crossed gluon is colour-connected to the quark, we obtain the following integrated antenna function:

$$
\begin{aligned}
& \mathcal{D}_{g, g g q}^{0}= \\
& +\frac{1}{\epsilon^{4}}[3 \delta(1-z)] \\
& +\frac{1}{\epsilon^{3}}\left[16+\frac{19}{3} \delta(1-z)-6 \mathcal{D}_{0}(z)-\frac{6}{z}-14 z+14 z^{2}\right] \\
& +\frac{1}{\epsilon^{2}}\left[\frac{85}{4}+\delta(1-z)\left(\frac{1429}{72}-\frac{7 \pi^{2}}{3}\right)-\frac{38}{3} \mathcal{D}_{0}(z)+12 \mathcal{D}_{1}(z)+26 \mathrm{H}(0 ; z)+32 \mathrm{H}(1 ; z)-\frac{12 \mathrm{H}(0 ; z)}{1-z}\right. \\
& \quad+z\left(-\frac{61}{2}-34 \mathrm{H}(0 ; z)-28 \mathrm{H}(1 ; z)\right)+\frac{1}{z}\left(-\frac{62}{3}-12 \mathrm{H}(0 ; z)-12 \mathrm{H}(1 ; z)\right)
\end{aligned}
$$




$$
\begin{aligned}
& \left.+z^{2}(31+28 \mathrm{H}(0 ; z)+28 \mathrm{H}(1 ; z))\right] \\
& +\frac{1}{\epsilon}\left[\frac{223}{4}-\frac{21 \pi^{2}}{2}+\delta(1-z)\left(\frac{23959}{432}-\frac{181 \pi^{2}}{36}-25 \zeta_{3}\right)+\left(-\frac{1429}{36}+\frac{14 \pi^{2}}{3}\right) \mathcal{D}_{0}(z)+\frac{76}{3} \mathcal{D}_{1}(z)-12 \mathcal{D}_{2}(z)\right. \\
& +\frac{595}{12} \mathrm{H}(0 ; z)+\frac{85}{2} \mathrm{H}(1 ; z)-2 \mathrm{H}(-1,0 ; z)+49 \mathrm{H}(0,0 ; z)+52 \mathrm{H}(0,1 ; z)+65 \mathrm{H}(1,0 ; z) \\
& +64 \mathrm{H}(1,1 ; z)-\frac{1}{1-z}\left(\frac{76}{3} \mathrm{H}(0 ; z)+24 \mathrm{H}(0,0 ; z)+24 \mathrm{H}(0,1 ; z)+24 \mathrm{H}(1,0 ; z)\right) \\
& +\frac{1}{z}\left(-\frac{1823}{36}+\frac{14 \pi^{2}}{3}-\frac{98}{3} \mathrm{H}(0 ; z)-\frac{124}{3} \mathrm{H}(1 ; z)-24 \mathrm{H}(0,0 ; z)-24 \mathrm{H}(0,1 ; z)-24 \mathrm{H}(1,0 ; z)\right. \\
& -24 \mathrm{H}(1,1 ; z))+z\left(-\frac{265}{4}+\frac{37 \pi^{2}}{3}-\frac{307}{6} \mathrm{H}(0 ; z)-61 \mathrm{H}(1 ; z)-4 \mathrm{H}(-1,0 ; z)-76 \mathrm{H}(0,0 ; z)\right. \\
& -68 \mathrm{H}(0,1 ; z)-58 \mathrm{H}(1,0 ; z)-56 \mathrm{H}(1,1 ; z))+z^{2}\left(\frac{565}{9}-11 \pi^{2}+\frac{230}{3} \mathrm{H}(0 ; z)+62 \mathrm{H}(1 ; z)\right. \\
& -4 \mathrm{H}(-1,0 ; z)+62 \mathrm{H}(0,0 ; z)+56 \mathrm{H}(0,1 ; z)+58 \mathrm{H}(1,0 ; z)+56 \mathrm{H}(1,1 ; z))] \\
& +\left[\frac{7793}{36}-\frac{1385 \pi^{2}}{72}-\frac{479 \zeta_{3}}{3}+\delta(1-z)\left(\frac{389623}{2592}-\frac{6857 \pi^{2}}{432}+\frac{163 \pi^{4}}{360}-\frac{1027 \zeta_{3}}{18}\right)\right. \\
& +\left(-\frac{23959}{216}+\frac{181 \pi^{2}}{18}+50 \zeta_{3}\right) \mathcal{D}_{0}(z)+\left(\frac{1429}{18}-\frac{28 \pi^{2}}{3}\right) \mathcal{D}_{1}(z)-\frac{76}{3} \mathcal{D}_{2}(z)+8 \mathcal{D}_{3}(z)+3 \pi^{2} \mathrm{H}(-1 ; z) \\
& +\frac{2801}{36} \mathrm{H}(0 ; z)-24 \pi^{2} \mathrm{H}(0 ; z)+\frac{223}{2} \mathrm{H}(1 ; z)-27 \pi^{2} \mathrm{H}(1 ; z)+10 \mathrm{H}(-1,0 ; z)+\frac{317}{4} \mathrm{H}(0,0 ; z) \\
& +\frac{595}{6} \mathrm{H}(0,1 ; z)+78 \mathrm{H}(1,0 ; z)+85 \mathrm{H}(1,1 ; z)+28 \mathrm{H}(-1,-1,0 ; z)-20 \mathrm{H}(-1,0,0 ; z) \\
& -4 \mathrm{H}(-1,0,1 ; z)-44 \mathrm{H}(0,-1,0 ; z)+95 \mathrm{H}(0,0,0 ; z)+98 \mathrm{H}(0,0,1 ; z)+94 \mathrm{H}(0,1,0 ; z) \\
& +104 \mathrm{H}(0,1,1 ; z)+101 \mathrm{H}(1,0,0 ; z)+130 \mathrm{H}(1,0,1 ; z)+118 \mathrm{H}(1,1,0 ; z)+128 \mathrm{H}(1,1,1 ; z) \\
& +\frac{1}{1-z}\left(\frac{5 \pi^{2}}{6}+16 \zeta_{3}-\frac{1501}{18} \mathrm{H}(0 ; z)+\frac{34}{3} \pi^{2} \mathrm{H}(0 ; z)-\frac{122}{3} \mathrm{H}(0,0 ; z)-\frac{152}{3} \mathrm{H}(0,1 ; z)\right. \\
& -\frac{137}{3} \mathrm{H}(1,0 ; z)+8 \mathrm{H}(0,-1,0 ; z)-48 \mathrm{H}(0,0,0 ; z)-48 \mathrm{H}(0,0,1 ; z)-44 \mathrm{H}(0,1,0 ; z) \\
& -48 \mathrm{H}(0,1,1 ; z)-44 \mathrm{H}(1,0,0 ; z)-48 \mathrm{H}(1,0,1 ; z)-48 \mathrm{H}(1,1,0 ; z))+z\left(-\frac{9395}{36}\right. \\
& +\frac{701 \pi^{2}}{36}+\frac{376 \zeta_{3}}{3}+4 \pi^{2} \mathrm{H}(-1 ; z)-\frac{6349}{36} \mathrm{H}(0 ; z)+\frac{101}{3} \pi^{2} \mathrm{H}(0 ; z)-\frac{265}{2} \mathrm{H}(1 ; z) \\
& +24 \pi^{2} \mathrm{H}(1 ; z)-\frac{201}{2} \mathrm{H}(0,0 ; z)-\frac{307}{3} \mathrm{H}(0,1 ; z)-130 \mathrm{H}(1,0 ; z)-122 \mathrm{H}(1,1 ; z) \\
& +32 \mathrm{H}(-1,-1,0 ; z)-28 \mathrm{H}(-1,0,0 ; z)-8 \mathrm{H}(-1,0,1 ; z)-160 \mathrm{H}(0,0,0 ; z) \\
& -152 \mathrm{H}(0,0,1 ; z)-128 \mathrm{H}(0,1,0 ; z)-136 \mathrm{H}(0,1,1 ; z)-94 \mathrm{H}(1,0,0 ; z)-116 \mathrm{H}(1,0,1 ; z) \\
& -104 \mathrm{H}(1,1,0 ; z)-112 \mathrm{H}(1,1,1 ; z))+\frac{1}{z}\left(-\frac{46025}{216}+\frac{151 \pi^{2}}{9}+54 \zeta_{3}+\frac{2}{3} \pi^{2} \mathrm{H}(-1 ; z)\right. \\
& -\frac{2179}{18} \mathrm{H}(0 ; z)+\frac{34}{3} \pi^{2} \mathrm{H}(0 ; z)-\frac{1823}{18} \mathrm{H}(1 ; z)+10 \pi^{2} \mathrm{H}(1 ; z)+\frac{68}{3} \mathrm{H}(-1,0 ; z)-\frac{196}{3} \mathrm{H}(0,0 ; z) \\
& -\frac{196}{3} \mathrm{H}(0,1 ; z)-\frac{239}{3} \mathrm{H}(1,0 ; z)-\frac{248}{3} \mathrm{H}(1,1 ; z)+8 \mathrm{H}(-1,-1,0 ; z)-4 \mathrm{H}(-1,0,0 ; z) \\
& +8 \mathrm{H}(0,-1,0 ; z)-48 \mathrm{H}(0,0,0 ; z)-48 \mathrm{H}(0,0,1 ; z)-44 \mathrm{H}(0,1,0 ; z)-48 \mathrm{H}(0,1,1 ; z) \\
& -36 \mathrm{H}(1,0,0 ; z)-48 \mathrm{H}(1,0,1 ; z)-44 \mathrm{H}(1,1,0 ; z)-48 \mathrm{H}(1,1,1 ; z))+z^{2}\left(\frac{6668}{27}-\frac{553 \pi^{2}}{18}\right.
\end{aligned}
$$




$$
\begin{aligned}
& -\frac{484 \zeta_{3}}{3}+\frac{10}{3} \pi^{2} \mathrm{H}(-1 ; z)+\frac{862}{9} \mathrm{H}(0 ; z)-28 \pi^{2} \mathrm{H}(0 ; z)+\frac{1130}{9} \mathrm{H}(1 ; z)-\frac{70}{3} \pi^{2} \mathrm{H}(1 ; z) \\
& +\frac{44}{3} \mathrm{H}(-1,0 ; z)+168 \mathrm{H}(0,0 ; z)+\frac{460}{3} \mathrm{H}(0,1 ; z)+124 \mathrm{H}(1,0 ; z)+124 \mathrm{H}(1,1 ; z) \\
& +24 \mathrm{H}(-1,-1,0 ; z)-24 \mathrm{H}(-1,0,0 ; z)-8 \mathrm{H}(-1,0,1 ; z)-32 \mathrm{H}(0,-1,0 ; z) \\
& +130 \mathrm{H}(0,0,0 ; z)+124 \mathrm{H}(0,0,1 ; z)+104 \mathrm{H}(0,1,0 ; z)+112 \mathrm{H}(0,1,1 ; z) \\
& +102 \mathrm{H}(1,0,0 ; z)+116 \mathrm{H}(1,0,1 ; z)+108 \mathrm{H}(1,1,0 ; z)+112 \mathrm{H}(1,1,1 ; z))] \\
& +
\end{aligned}
$$

while the crossing the middle gluon (which is colour-connected to the other two gluons) results in:

$$
\begin{aligned}
& \mathcal{D}_{g^{\prime}, g g q}^{0}= \\
& +\frac{1}{\epsilon^{4}}[4 \delta(1-z)] \\
& +\frac{1}{\epsilon^{3}}\left[18+6 \delta(1-z)-8 \mathcal{D}_{0}(z)-\frac{8}{z}-12 z+12 z^{2}\right] \\
& +\frac{1}{\epsilon^{2}}\left[\frac{45}{2}+\delta(1-z)\left(\frac{75}{4}-\frac{10 \pi^{2}}{3}\right)-12 \mathcal{D}_{0}(z)+16 \mathcal{D}_{1}(z)+26 \mathrm{H}(0 ; z)-\frac{16 \mathrm{H}(0 ; z)}{1-z}+\frac{1}{z}(-28\right. \\
& -16 \mathrm{H}(0 ; z)-16 \mathrm{H}(1 ; z))+36 \mathrm{H}(1 ; z)+z(-29-40 \mathrm{H}(0 ; z)-24 \mathrm{H}(1 ; z)) \\
& \left.+z^{2}(34+24 \mathrm{H}(0 ; z)+24 \mathrm{H}(1 ; z))\right] \\
& +\frac{1}{\epsilon}\left[\frac{85}{2}-\frac{40 \pi^{2}}{3}+\delta(1-z)\left(\frac{417}{8}-5 \pi^{2}-\frac{116 \zeta_{3}}{3}\right)+\left(-\frac{75}{2}+\frac{20 \pi^{2}}{3}\right) \mathcal{D}_{0}(z)+24 \mathcal{D}_{1}(z)-16 \mathcal{D}_{2}(z)\right. \\
& +\frac{133}{2} \mathrm{H}(0 ; z)+45 \mathrm{H}(1 ; z)-16 \mathrm{H}(-1,0 ; z)+56 \mathrm{H}(0,0 ; z)+52 \mathrm{H}(0,1 ; z)+70 \mathrm{H}(1,0 ; z) \\
& +72 \mathrm{H}(1,1 ; z)+\frac{1}{1-z}(-24 \mathrm{H}(0 ; z)-36 \mathrm{H}(0,0 ; z)-32 \mathrm{H}(0,1 ; z)-32 \mathrm{H}(1,0 ; z)) \\
& +\frac{1}{1+z}\left(\frac{2 \pi^{2}}{3}+8 \mathrm{H}(-1,0 ; z)-4 \mathrm{H}(0,0 ; z)\right)+\frac{1}{z}\left(-\frac{785}{18}+6 \pi^{2}-\frac{116}{3} \mathrm{H}(0 ; z)-56 \mathrm{H}(1 ; z)\right. \\
& -8 \mathrm{H}(-1,0 ; z)-32 \mathrm{H}(0,0 ; z)-32 \mathrm{H}(0,1 ; z)-32 \mathrm{H}(1,0 ; z)-32 \mathrm{H}(1,1 ; z))+z\left(-\frac{59}{2}\right. \\
& +\frac{46 \pi^{2}}{3}-29 \mathrm{H}(0 ; z)-58 \mathrm{H}(1 ; z)-8 \mathrm{H}(-1,0 ; z)-92 \mathrm{H}(0,0 ; z)-80 \mathrm{H}(0,1 ; z) \\
& -44 \mathrm{H}(1,0 ; z)-48 \mathrm{H}(1,1 ; z))+z^{2}\left(\frac{280}{9}-\frac{34 \pi^{2}}{3}+\frac{292}{3} \mathrm{H}(0 ; z)+68 \mathrm{H}(1 ; z)\right. \\
& -8 \mathrm{H}(-1,0 ; z)+52 \mathrm{H}(0,0 ; z)+48 \mathrm{H}(0,1 ; z)+44 \mathrm{H}(1,0 ; z)+48 \mathrm{H}(1,1 ; z))] \\
& +\left[\frac{3493}{18}-\frac{1121 \pi^{2}}{36}-274 \zeta_{3}+\delta(1-z)\left(\frac{2275}{16}-\frac{125 \pi^{2}}{8}+\frac{61 \pi^{4}}{90}-64 \zeta_{3}\right)+\mathcal{D}_{0}(z)\left(-\frac{417}{4}+10 \pi^{2}\right.\right. \\
& \left.+\frac{232 \zeta_{3}}{3}\right)+\left(75-\frac{40 \pi^{2}}{3}\right) \mathcal{D}_{1}(z)-24 \mathcal{D}_{2}(z)+\frac{32}{3} \mathcal{D}_{3}(z)+\frac{26}{3} \pi^{2} \mathrm{H}(-1 ; z)+\frac{137}{6} \mathrm{H}(0 ; z) \\
& -\frac{89}{3} \pi^{2} \mathrm{H}(0 ; z)+85 \mathrm{H}(1 ; z)-\frac{86}{3} \pi^{2} \mathrm{H}(1 ; z)+\frac{1055}{6} \mathrm{H}(0,0 ; z)+133 \mathrm{H}(0,1 ; z) \\
& +\frac{262}{3} \mathrm{H}(1,0 ; z)+90 \mathrm{H}(1,1 ; z)+40 \mathrm{H}(-1,-1,0 ; z)-68 \mathrm{H}(-1,0,0 ; z)-32 \mathrm{H}(-1,0,1 ; z)
\end{aligned}
$$




$$
\begin{aligned}
& -36 \mathrm{H}(0,-1,0 ; z)+116 \mathrm{H}(0,0,0 ; z)+112 \mathrm{H}(0,0,1 ; z)+84 \mathrm{H}(0,1,0 ; z)+104 \mathrm{H}(0,1,1 ; z) \\
& +146 \mathrm{H}(1,0,0 ; z)+140 \mathrm{H}(1,0,1 ; z)+148 \mathrm{H}(1,1,0 ; z)+144 \mathrm{H}(1,1,1 ; z) \\
& +\frac{1}{1-z}\left(+24 \zeta_{3}-79 \mathrm{H}(0 ; z)+\frac{52}{3} \pi^{2} \mathrm{H}(0 ; z)-\frac{166}{3} \mathrm{H}(0,0 ; z)-48 \mathrm{H}(0,1 ; z)-48 \mathrm{H}(1,0 ; z)\right. \\
& -76 \mathrm{H}(0,0,0 ; z)-72 \mathrm{H}(0,0,1 ; z)-56 \mathrm{H}(0,1,0 ; z)-64 \mathrm{H}(0,1,1 ; z)-64 \mathrm{H}(1,0,0 ; z) \\
& -64 \mathrm{H}(1,0,1 ; z)-64 \mathrm{H}(1,1,0 ; z))+\frac{1}{1+z}\left(+28 \zeta_{3}-\frac{16}{3} \pi^{2} \mathrm{H}(-1 ; z)+\frac{4}{3} \pi^{2} \mathrm{H}(0 ; z)\right. \\
& -32 \mathrm{H}(-1,-1,0 ; z)+40 \mathrm{H}(-1,0,0 ; z)+16 \mathrm{H}(-1,0,1 ; z)+24 \mathrm{H}(0,-1,0 ; z) \\
& -12 \mathrm{H}(0,0,0 ; z)-8 \mathrm{H}(0,0,1 ; z))+\frac{1}{z}\left(-\frac{8407}{36}+\frac{206 \pi^{2}}{9}+\frac{14}{3} \pi^{2} \mathrm{H}(-1 ; z)-\frac{1141}{9} \mathrm{H}(0 ; z)\right. \\
& +16 \pi^{2} \mathrm{H}(0 ; z)-\frac{785}{9} \mathrm{H}(1 ; z)+\frac{38}{3} \pi^{2} \mathrm{H}(1 ; z)-\frac{20}{3} \mathrm{H}(-1,0 ; z)-\frac{232}{3} \mathrm{H}(0,0 ; z) \\
& -\frac{232}{3} \mathrm{H}(0,1 ; z)-\frac{314}{3} \mathrm{H}(1,0 ; z)-112 \mathrm{H}(1,1 ; z)+24 \mathrm{H}(-1,-1,0 ; z)-36 \mathrm{H}(-1,0,0 ; z) \\
& -16 \mathrm{H}(-1,0,1 ; z)-24 \mathrm{H}(0,-1,0 ; z)-64 \mathrm{H}(0,0,0 ; z)-64 \mathrm{H}(0,0,1 ; z)-56 \mathrm{H}(0,1,0 ; z) \\
& -64 \mathrm{H}(0,1,1 ; z)-72 \mathrm{H}(1,0,0 ; z)-64 \mathrm{H}(1,0,1 ; z)-68 \mathrm{H}(1,1,0 ; z)-64 \mathrm{H}(1,1,1 ; z) \\
& \left.+\frac{256 \zeta_{3}}{3}\right)+z\left(-\frac{3571}{18}+\frac{377 \pi^{2}}{18}+188 \zeta_{3}+\frac{10}{3} \pi^{2} \mathrm{H}(-1 ; z)-\frac{857}{6} \mathrm{H}(0 ; z)+\frac{128}{3} \pi^{2} \mathrm{H}(0 ; z)\right. \\
& -59 \mathrm{H}(1 ; z)+\frac{58}{3} \pi^{2} \mathrm{H}(1 ; z)+36 \mathrm{H}(-1,0 ; z)-\frac{191}{3} \mathrm{H}(0,0 ; z)-58 \mathrm{H}(0,1 ; z) \\
& -\frac{344}{3} \mathrm{H}(1,0 ; z)-116 \mathrm{H}(1,1 ; z)+8 \mathrm{H}(-1,-1,0 ; z)-28 \mathrm{H}(-1,0,0 ; z)-16 \mathrm{H}(-1,0,1 ; z) \\
& -196 \mathrm{H}(0,0,0 ; z)-184 \mathrm{H}(0,0,1 ; z)-144 \mathrm{H}(0,1,0 ; z)-160 \mathrm{H}(0,1,1 ; z)-76 \mathrm{H}(1,0,0 ; z) \\
& -88 \mathrm{H}(1,0,1 ; z)-92 \mathrm{H}(1,1,0 ; z)-96 \mathrm{H}(1,1,1 ; z))+z^{2}\left(\frac{2026}{9}-\frac{343 \pi^{2}}{9}-184 \zeta_{3}\right. \\
& +4 \pi^{2} \mathrm{H}(-1 ; z)+\frac{8}{3} \mathrm{H}(0 ; z)-\frac{80}{3} \pi^{2} \mathrm{H}(0 ; z)+\frac{560}{9} \mathrm{H}(1 ; z)-20 \pi^{2} \mathrm{H}(1 ; z)+\frac{88}{3} \mathrm{H}(-1,0 ; z) \\
& +224 \mathrm{H}(0,0 ; z)+\frac{584}{3} \mathrm{H}(0,1 ; z)+136 \mathrm{H}(1,0 ; z)+136 \mathrm{H}(1,1 ; z)+16 \mathrm{H}(-1,-1,0 ; z) \\
& -32 \mathrm{H}(-1,0,0 ; z)-16 \mathrm{H}(-1,0,1 ; z)-32 \mathrm{H}(0,-1,0 ; z)+108 \mathrm{H}(0,0,0 ; z) \\
& +104 \mathrm{H}(0,0,1 ; z)+80 \mathrm{H}(0,1,0 ; z)+96 \mathrm{H}(0,1,1 ; z)+68 \mathrm{H}(1,0,0 ; z)+88 \mathrm{H}(1,0,1 ; z) \\
& +88 \mathrm{H}(1,1,0 ; z)+96 \mathrm{H}(1,1,1 ; z))] \\
& +\mathcal{O}(\epsilon) .
\end{aligned}
$$

Crossing the gluon in the $E_{4}^{0}$-type antenna functions into the initial state results in $\left(g, q q^{\prime} \bar{q}^{\prime}\right)$-antenna at leading and subleading colour, which integrate to:

$$
\begin{aligned}
& \mathcal{E}_{g, q q^{\prime} \bar{q}^{\prime}}^{0}= \\
+ & \frac{1}{\epsilon^{3}}\left[-\frac{1}{3} \delta(1-z)\right] \\
+ & \frac{1}{\epsilon^{2}}\left[-\frac{3}{2}-\frac{19}{18} \delta(1-z)+\frac{2}{3} \mathcal{D}_{0}(z)+\mathrm{H}(0 ; z)+\frac{4}{3 z}+z\left(\frac{3}{2}+\mathrm{H}(0 ; z)\right)-\frac{8}{3} z^{2}\right] \\
+ & \frac{1}{\epsilon}\left[-\frac{7}{3}-\frac{\pi^{2}}{3}+\delta(1-z)\left(-\frac{373}{108}+\frac{5 \pi^{2}}{18}\right)+\frac{19}{9} \mathcal{D}_{0}(z)-\frac{4}{3} \mathcal{D}_{1}(z)-\frac{5}{6} \mathrm{H}(0 ; z)+\frac{4}{3(1-z)} \mathrm{H}(0 ; z)\right.
\end{aligned}
$$




$$
\begin{aligned}
& -3 \mathrm{H}(1 ; z)+3 \mathrm{H}(0,0 ; z)+2 \mathrm{H}(0,1 ; z)+\frac{1}{z}\left(\frac{52}{9}+\frac{8}{3} \mathrm{H}(0 ; z)+\frac{8}{3} \mathrm{H}(1 ; z)\right)+z\left(\frac{13}{3}-\frac{\pi^{2}}{3}\right. \\
& \left.\left.+\frac{25}{6} \mathrm{H}(0 ; z)+3 \mathrm{H}(1 ; z)+3 \mathrm{H}(0,0 ; z)+2 \mathrm{H}(0,1 ; z)\right)-z^{2}\left(8+\frac{16}{3} \mathrm{H}(0 ; z)+\frac{16}{3} \mathrm{H}(1 ; z)\right)\right] \\
+[- & \frac{17}{6}+\frac{19 \pi^{2}}{36}-2 \zeta_{3}+\delta(1-z)\left(-\frac{7081}{648}+\frac{95 \pi^{2}}{108}+\frac{32 \zeta_{3}}{9}\right)+\left(\frac{373}{54}-\frac{5 \pi^{2}}{9}\right) \mathcal{D}_{0}(z)-\frac{38}{9} \mathcal{D}_{1}(z) \\
& +\frac{4}{3} \mathcal{D}_{2}(z)+\frac{43}{9} \mathrm{H}(0 ; z)-\frac{7}{6} \pi^{2} \mathrm{H}(0 ; z)-\frac{14}{3} \mathrm{H}(1 ; z)+\frac{1}{2} \mathrm{H}(0,0 ; z)-\frac{5}{3} \mathrm{H}(0,1 ; z)-6 \mathrm{H}(1,0 ; z) \\
& -6 \mathrm{H}(1,1 ; z)+7 \mathrm{H}(0,0,0 ; z)+6 \mathrm{H}(0,0,1 ; z)+4 \mathrm{H}(0,1,0 ; z)+4 \mathrm{H}(0,1,1 ; z) \\
& +\frac{1}{1-z}\left(\frac{38}{9} \mathrm{H}(0 ; z)+\frac{8}{3} \mathrm{H}(0,0 ; z)+\frac{8}{3} \mathrm{H}(0,1 ; z)+\frac{8}{3} \mathrm{H}(1,0 ; z)\right)+\frac{1}{z}\left(23-\frac{10 \pi^{2}}{9}\right. \\
& \left.+\frac{104}{9} \mathrm{H}(0 ; z)+\frac{104}{9} \mathrm{H}(1 ; z)+\frac{16}{3} \mathrm{H}(0,0 ; z)+\frac{16}{3} \mathrm{H}(0,1 ; z)+\frac{16}{3} \mathrm{H}(1,0 ; z)+\frac{16}{3} \mathrm{H}(1,1 ; z)\right) \\
& +z\left(\frac{16}{3}-\frac{59 \pi^{2}}{36}-2 \zeta_{3}+\frac{115}{9} \mathrm{H}(0 ; z)-\frac{7}{6} \pi^{2} \mathrm{H}(0 ; z)+\frac{26}{3} \mathrm{H}(1 ; z)+\frac{19}{2} \mathrm{H}(0,0 ; z)\right. \\
& +\frac{25}{3} \mathrm{H}(0,1 ; z)+6 \mathrm{H}(1,0 ; z)+6 \mathrm{H}(1,1 ; z)+7 \mathrm{H}(0,0,0 ; z)+6 \mathrm{H}(0,0,1 ; z)+4 \mathrm{H}(0,1,0 ; z) \\
& +4 \mathrm{H}(0,1,1 ; z))+z^{2}\left(-\frac{580}{27}+\frac{20 \pi^{2}}{9}-16 \mathrm{H}(0 ; z)-16 \mathrm{H}(1 ; z)-\frac{32}{3} \mathrm{H}(0,0 ; z)\right. \\
& \left.\left.-\frac{32}{3} \mathrm{H}(0,1 ; z)-\frac{32}{3} \mathrm{H}(1,0 ; z)-\frac{32}{3} \mathrm{H}(1,1 ; z)\right)\right] \\
+ & \mathcal{O}(\epsilon)
\end{aligned}
$$

and

$$
\begin{aligned}
& \tilde{\mathcal{E}}_{g, q q^{\prime} q^{\prime}}^{0}= \\
& +\frac{1}{\epsilon^{2}}\left[1+2 \mathrm{H}(0 ; z)+\frac{4}{3 z}+z(-1+2 \mathrm{H}(0 ; z))-\frac{4}{3} z^{2}\right] \\
& +\frac{1}{\epsilon}\left[\frac{10}{3}-\frac{2 \pi^{2}}{3}+5 \mathrm{H}(0 ; z)+2 \mathrm{H}(1 ; z)+\frac{1}{z}\left(\frac{44}{9}+\frac{8}{3} \mathrm{H}(0 ; z)+\frac{8}{3} \mathrm{H}(1 ; z)\right)+6 \mathrm{H}(0,0 ; z)\right. \\
& \quad+4 \mathrm{H}(0,1 ; z)+z\left(-\frac{16}{3}-\frac{2 \pi^{2}}{3}-\mathrm{H}(0 ; z)-2 \mathrm{H}(1 ; z)+6 \mathrm{H}(0,0 ; z)+4 \mathrm{H}(0,1 ; z)\right) \\
& \left.\quad+z^{2}\left(-\frac{26}{9}-\frac{8}{3} \mathrm{H}(0 ; z)-\frac{8}{3} \mathrm{H}(1 ; z)\right)\right] \\
& +\frac{200}{9}-\frac{11 \pi^{2}}{6}-4 \zeta_{3}+\frac{68}{3} \mathrm{H}(0 ; z)-\frac{7}{3} \pi^{2} \mathrm{H}(0 ; z)+\frac{20}{3} \mathrm{H}(1 ; z)+13 \mathrm{H}(0,0 ; z)+10 \mathrm{H}(0,1 ; z) \\
& \quad+4 \mathrm{H}(1,0 ; z)+14 \mathrm{H}(0,0,0 ; z)+12 \mathrm{H}(0,0,1 ; z)+8 \mathrm{H}(0,1,0 ; z)+8 \mathrm{H}(0,1,1 ; z) \\
& \quad+4 \mathrm{H}(1,1 ; z)+\frac{1}{z}\left(\frac{457}{27}-\frac{10 \pi^{2}}{9}+\frac{88}{9} \mathrm{H}(0 ; z)+\frac{88}{9} \mathrm{H}(1 ; z)+\frac{16}{3} \mathrm{H}(0,0 ; z)+\frac{16}{3} \mathrm{H}(0,1 ; z)\right. \\
& \left.\quad+\frac{16}{3} \mathrm{H}(1,0 ; z)+\frac{16}{3} \mathrm{H}(1,1 ; z)\right)+z\left(-\frac{299}{9}+\frac{\pi^{2}}{2}-\frac{14}{3} \mathrm{H}(0 ; z)-\frac{7}{3} \pi^{2} \mathrm{H}(0 ; z)-\frac{32}{3} \mathrm{H}(1 ; z)\right. \\
& \quad-\mathrm{H}(0,0 ; z)-2 \mathrm{H}(0,1 ; z)-4 \mathrm{H}(1,0 ; z)-4 \mathrm{H}(1,1 ; z)+14 \mathrm{H}(0,0,0 ; z)+12 \mathrm{H}(0,0,1 ; z) \\
& \left.\quad+8 \mathrm{H}(0,1,0 ; z)+8 \mathrm{H}(0,1,1 ; z)-4 \zeta_{3}\right)+z^{2}\left(-\frac{160}{27}+\frac{10 \pi^{2}}{9}-\frac{52}{9} \mathrm{H}(0 ; z)-\frac{52}{9} \mathrm{H}(1 ; z)\right. \\
& \left.\left.\quad-\frac{16}{3} \mathrm{H}(0,0 ; z)-\frac{16}{3} \mathrm{H}(0,1 ; z)-\frac{16}{3} \mathrm{H}(1,0 ; z)-\frac{16}{3} \mathrm{H}(1,1 ; z)\right)\right]
\end{aligned}
$$


The integrals of the one-loop virtual corrections to the $(g, g q)$ antenna functions at leading colour and for a closed quark loop yield:

$$
\begin{aligned}
& \mathcal{D}_{g, g q}^{1}= \\
& +\frac{1}{\epsilon^{4}}\left[-\frac{1}{4} \delta(1-z)\right] \\
& +\frac{1}{\epsilon^{3}}\left[-\frac{5}{4}-\frac{53}{24} \delta(1-z)+\frac{1}{2} \mathcal{D}_{0}(z)+\frac{1}{2 z}+z-z^{2}\right] \\
& +\frac{1}{\epsilon^{2}}\left[-\frac{13}{3}+\delta(1-z)\left(-3+\frac{7 \pi^{2}}{24}\right)+\frac{31}{12} \mathcal{D}_{0}(z)-\mathcal{D}_{1}(z)-5 \mathrm{H}(0 ; z)-\frac{5}{2} \mathrm{H}(1 ; z)+\frac{2 \mathrm{H}(0 ; z)}{1-z}+\frac{1}{z}\left(\frac{31}{12}\right.\right. \\
& \left.+2 \mathrm{H}(0 ; z)+\mathrm{H}(1 ; z))+z\left(\frac{11}{3}+4 \mathrm{H}(0 ; z)+2 \mathrm{H}(1 ; z)\right)+z^{2}\left(-\frac{11}{3}-4 \mathrm{H}(0 ; z)-2 \mathrm{H}(1 ; z)\right)\right] \\
& +\frac{1}{\epsilon}\left[-\frac{1}{12}+\frac{35 \pi^{2}}{24}+\delta(1-z)\left(-\frac{197}{24}+\frac{39 \pi^{2}}{16}+\frac{11 \zeta_{3}}{3}\right)+\left(\frac{37}{8}-\frac{7 \pi^{2}}{12}\right) \mathcal{D}_{0}(z)-\frac{10}{3} \mathcal{D}_{1}(z)+\mathcal{D}_{2}(z)\right. \\
& -\frac{43}{12} \mathrm{H}(0 ; z)-\frac{49}{12} \mathrm{H}(1 ; z)-15 \mathrm{H}(0,0 ; z)-\frac{15}{2} \mathrm{H}(0,1 ; z)-\frac{15}{2} \mathrm{H}(1,0 ; z)-5 \mathrm{H}(1,1 ; z) \\
& +\frac{1}{1-z}\left(\frac{29}{6} \mathrm{H}(0 ; z)+6 \mathrm{H}(0,0 ; z)+3 \mathrm{H}(0,1 ; z)+3 \mathrm{H}(1,0 ; z)\right)+\frac{1}{z}\left(\frac{37}{8}-\frac{7 \pi^{2}}{12}+\frac{29}{6} \mathrm{H}(0 ; z)\right. \\
& \left.+\frac{10}{3} \mathrm{H}(1 ; z)+6 \mathrm{H}(0,0 ; z)+3 \mathrm{H}(0,1 ; z)+3 \mathrm{H}(1,0 ; z)+2 \mathrm{H}(1,1 ; z)\right)+z\left(\frac{1}{12}-\frac{7 \pi^{2}}{6}\right. \\
& \left.+\frac{11}{3} \mathrm{H}(0 ; z)+\frac{11}{3} \mathrm{H}(1 ; z)+12 \mathrm{H}(0,0 ; z)+6 \mathrm{H}(0,1 ; z)+6 \mathrm{H}(1,0 ; z)+4 \mathrm{H}(1,1 ; z)\right) \\
& \left.+z^{2}\left(\frac{7 \pi^{2}}{6}-\frac{11}{3} \mathrm{H}(0 ; z)-\frac{11}{3} \mathrm{H}(1 ; z)-12 \mathrm{H}(0,0 ; z)-6 \mathrm{H}(0,1 ; z)-6 \mathrm{H}(1,0 ; z)-4 \mathrm{H}(1,1 ; z)\right)\right] \\
& +\left[-7+\frac{241 \pi^{2}}{48}+\frac{125 \zeta_{3}}{6}+\delta(1-z)\left(-\frac{239}{12}+\frac{283 \pi^{2}}{96}-\frac{11 \pi^{4}}{144}+\frac{793 \zeta_{3}}{36}\right)+\left(\frac{317}{24}-\frac{11 \pi^{2}}{4}\right.\right. \\
& \left.-\frac{22 \zeta_{3}}{3}\right) \mathcal{D}_{0}(z)+\left(-\frac{63}{8}+\frac{7 \pi^{2}}{6}\right) \mathcal{D}_{1}(z)+\frac{29}{12} \mathcal{D}_{2}(z)-\frac{2}{3} \mathcal{D}_{3}(z)-\frac{37}{12} \mathrm{H}(0 ; z)+\frac{35}{6} \pi^{2} \mathrm{H}(0 ; z)-\frac{13}{12} \mathrm{H}(1 ; z) \\
& +\frac{10}{3} \pi^{2} \mathrm{H}(1 ; z)-\frac{19}{12} \mathrm{H}(0,0 ; z)-\frac{37}{12} \mathrm{H}(0,1 ; z)-\frac{37}{12} \mathrm{H}(1,0 ; z)-\frac{43}{12} \mathrm{H}(1,1 ; z) \\
& -35 \mathrm{H}(0,0,0 ; z)-20 \mathrm{H}(0,0,1 ; z)-15 \mathrm{H}(0,1,0 ; z)-\frac{25}{2} \mathrm{H}(0,1,1 ; z)-15 \mathrm{H}(1,0,0 ; z) \\
& -\frac{25}{2} \mathrm{H}(1,0,1 ; z)-10 \mathrm{H}(1,1,0 ; z)-10 \mathrm{H}(1,1,1 ; z)+\frac{1}{1-z}\left(\frac{\pi^{2}}{4}-\zeta_{3}+\frac{91}{8} \mathrm{H}(0 ; z)\right. \\
& -\frac{7}{3} \pi^{2} \mathrm{H}(0 ; z)-\frac{1}{6} \pi^{2} \mathrm{H}(1 ; z)+\frac{37}{3} \mathrm{H}(0,0 ; z)+\frac{19}{3} \mathrm{H}(0,1 ; z)+\frac{47}{6} \mathrm{H}(1,0 ; z)+14 \mathrm{H}(0,0,0 ; z) \\
& +8 \mathrm{H}(0,0,1 ; z)+6 \mathrm{H}(0,1,0 ; z)+5 \mathrm{H}(0,1,1 ; z)+6 \mathrm{H}(1,0,0 ; z)+5 \mathrm{H}(1,0,1 ; z) \\
& +4 \mathrm{H}(1,1,0 ; z))+\frac{1}{z}\left(\frac{107}{8}-\frac{7 \pi^{2}}{2}-\frac{25 \zeta_{3}}{3}+\frac{91}{8} \mathrm{H}(0 ; z)-\frac{7}{3} \pi^{2} \mathrm{H}(0 ; z)+\frac{63}{8} \mathrm{H}(1 ; z)\right. \\
& -\frac{4}{3} \pi^{2} \mathrm{H}(1 ; z)+\frac{28}{3} \mathrm{H}(0,0 ; z)+\frac{19}{3} \mathrm{H}(0,1 ; z)+\frac{29}{6} \mathrm{H}(1,0 ; z)+\frac{29}{6} \mathrm{H}(1,1 ; z) \\
& +14 \mathrm{H}(0,0,0 ; z)+8 \mathrm{H}(0,0,1 ; z)+6 \mathrm{H}(0,1,0 ; z)+5 \mathrm{H}(0,1,1 ; z)+6 \mathrm{H}(1,0,0 ; z) \\
& +5 \mathrm{H}(1,0,1 ; z)+4 \mathrm{H}(1,1,0 ; z)+4 \mathrm{H}(1,1,1 ; z))+z\left(\frac{11}{36}-\frac{17 \pi^{2}}{4}-\frac{50 \zeta_{3}}{3}+\frac{1}{6} \mathrm{H}(0 ; z)\right.
\end{aligned}
$$




$$
\begin{aligned}
& -\frac{14}{3} \pi^{2} \mathrm{H}(0 ; z)+\frac{1}{12} \mathrm{H}(1 ; z)-\frac{8}{3} \pi^{2} \mathrm{H}(1 ; z)+\frac{11}{3} \mathrm{H}(0,0 ; z)+\frac{11}{3} \mathrm{H}(0,1 ; z)+\frac{11}{3} \mathrm{H}(1,0 ; z) \\
& +\frac{11}{3} \mathrm{H}(1,1 ; z)+28 \mathrm{H}(0,0,0 ; z)+16 \mathrm{H}(0,0,1 ; z)+12 \mathrm{H}(0,1,0 ; z)+10 \mathrm{H}(0,1,1 ; z) \\
& +12 \mathrm{H}(1,0,0 ; z)+10 \mathrm{H}(1,0,1 ; z)+8 \mathrm{H}(1,1,0 ; z)+8 \mathrm{H}(1,1,1 ; z))+z^{2}\left(\frac{17 \pi^{2}}{4}+\frac{50 \zeta_{3}}{3}\right. \\
& +\frac{14}{3} \pi^{2} \mathrm{H}(0 ; z)+\frac{8}{3} \pi^{2} \mathrm{H}(1 ; z)-\frac{11}{3} \mathrm{H}(0,0 ; z)-\frac{11}{3} \mathrm{H}(0,1 ; z)-\frac{11}{3} \mathrm{H}(1,0 ; z)-\frac{11}{3} \mathrm{H}(1,1 ; z) \\
& -28 \mathrm{H}(0,0,0 ; z)-16 \mathrm{H}(0,0,1 ; z)-12 \mathrm{H}(0,1,0 ; z)-10 \mathrm{H}(0,1,1 ; z)-12 \mathrm{H}(1,0,0 ; z) \\
& -10 \mathrm{H}(1,0,1 ; z)-8 \mathrm{H}(1,1,0 ; z)-8 \mathrm{H}(1,1,1 ; z))] \\
& +\mathcal{O}(\epsilon)
\end{aligned}
$$

and

$$
\begin{aligned}
& \hat{\mathcal{D}}_{g, g q}^{1}= \\
&+\frac{1}{\epsilon^{3}}\left[\frac{1}{3} \delta(1-z)\right] \\
&+\frac{1}{\epsilon^{2}}\left[\frac{5}{6}+\frac{1}{4} \delta(1-z)-\frac{1}{3} \mathcal{D}_{0}(z)-\frac{1}{3 z}-\frac{2}{3} z+\frac{2}{3} z^{2}\right] \\
&+\frac{1}{\epsilon}\left[-\frac{1}{6}+\delta(1-z)\left(\frac{7}{12}-\frac{\pi^{2}}{4}\right)-\frac{1}{4} \mathcal{D}_{0}(z)+\frac{1}{3} \mathcal{D}_{1}(z)+\frac{5}{6} \mathrm{H}(0 ; z)-\frac{\mathrm{H}(0 ; z)}{3(1-z)}+\frac{1}{z}\left(-\frac{1}{4}-\frac{1}{3} \mathrm{H}(0 ; z)\right.\right. \\
&\left.\left.\quad-\frac{1}{3} \mathrm{H}(1 ; z)\right)+z\left(-\frac{1}{12}-\frac{2}{3} \mathrm{H}(0 ; z)-\frac{2}{3} \mathrm{H}(1 ; z)\right)+z^{2}\left(\frac{2}{3} \mathrm{H}(0 ; z)+\frac{2}{3} \mathrm{H}(1 ; z)\right)+\frac{5}{6} \mathrm{H}(1 ; z)\right] \\
&+\left[\frac{1}{2}-\frac{5 \pi^{2}}{8}+\delta(1-z)\left(\frac{7}{6}-\frac{3 \pi^{2}}{16}-\frac{7 \zeta_{3}}{9}\right)+\left(-\frac{7}{12}+\frac{\pi^{2}}{4}\right) \mathcal{D}_{0}(z)+\frac{1}{4} \mathcal{D}_{1}(z)-\frac{1}{6} \mathcal{D}_{2}(z)-\frac{1}{6} \mathrm{H}(0 ; z)\right. \\
& \quad-\frac{1}{6} \mathrm{H}(1 ; z)+\frac{5}{6} \mathrm{H}(0,0 ; z)+\frac{5}{6} \mathrm{H}(0,1 ; z)+\frac{5}{6} \mathrm{H}(1,0 ; z)+\frac{5}{6} \mathrm{H}(1,1 ; z)+\frac{1}{1-z}\left(-\frac{1}{4} \mathrm{H}(0 ; z)\right. \\
&\left.\quad-\frac{1}{3} \mathrm{H}(0,0 ; z)-\frac{1}{3} \mathrm{H}(0,1 ; z)-\frac{1}{3} \mathrm{H}(1,0 ; z)\right)+\frac{1}{z}\left(-\frac{3}{4}+\frac{\pi^{2}}{4}-\frac{1}{4} \mathrm{H}(0 ; z)-\frac{1}{4} \mathrm{H}(1 ; z)\right. \\
&\left.\quad-\frac{1}{3} \mathrm{H}(0,0 ; z)-\frac{1}{3} \mathrm{H}(0,1 ; z)-\frac{1}{3} \mathrm{H}(1,0 ; z)-\frac{1}{3} \mathrm{H}(1,1 ; z)\right)+z\left(-\frac{7}{18}+\frac{\pi^{2}}{2}-\frac{1}{6} \mathrm{H}(0 ; z)\right. \\
&\left.\quad-\frac{1}{12} \mathrm{H}(1 ; z)-\frac{2}{3} \mathrm{H}(0,0 ; z)-\frac{2}{3} \mathrm{H}(0,1 ; z)-\frac{2}{3} \mathrm{H}(1,0 ; z)-\frac{2}{3} \mathrm{H}(1,1 ; z)\right)+z^{2}\left(-\frac{\pi^{2}}{2}\right. \\
&\left.\left.\quad+\frac{2}{3} \mathrm{H}(0,0 ; z)+\frac{2}{3} \mathrm{H}(0,1 ; z)+\frac{2}{3} \mathrm{H}(1,0 ; z)+\frac{2}{3} \mathrm{H}(1,1 ; z)\right)\right] \\
&+ \mathcal{O}(\epsilon) .
\end{aligned}
$$

\subsection{Gluon-gluon antennae}

The NLO gluon-gluon antenna functions crossed to initial-final kinematics integrate to:

$$
\begin{aligned}
& \mathcal{F}_{g, g g}^{0}= \\
+ & \frac{1}{\epsilon^{2}}[2 \delta(1-z)] \\
+ & \frac{1}{\epsilon}\left[4+\frac{11}{6} \delta(1-z)-\frac{2}{z}-2 z+2 z^{2}-2 \mathcal{D}_{0}(z)\right]
\end{aligned}
$$




$$
\begin{aligned}
& +\left[\delta(1-z)\left(\frac{67}{18}-\frac{\pi^{2}}{2}\right)-\frac{11}{6} \mathcal{D}_{0}(z)+2 \mathcal{D}_{1}(z)+4 \mathrm{H}(0 ; z)+4 \mathrm{H}(1 ; z)-\frac{2}{1-z} \mathrm{H}(0 ; z)\right. \\
& \left.-\frac{1}{z}\left(\frac{11}{6}+2 \mathrm{H}(0 ; z)+2 \mathrm{H}(1 ; z)\right)+\left(-z+z^{2}\right)(2 \mathrm{H}(0 ; z)+2 \mathrm{H}(1 ; z))\right] \\
& +\epsilon\left[2-\pi^{2}+\delta(1-z)\left(\frac{202}{27}-\frac{11 \pi^{2}}{24}-\frac{14 \zeta_{3}}{3}\right)+\left(-\frac{67}{18}+\frac{\pi^{2}}{2}\right) \mathcal{D}_{0}(z)+\frac{11}{6} \mathcal{D}_{1}(z)-\mathcal{D}_{2}(z)+4 \mathrm{H}(0,0 ; z)\right. \\
& +4 \mathrm{H}(0,1 ; z)+4 \mathrm{H}(1,0 ; z)+4 \mathrm{H}(1,1 ; z)+\frac{1}{1-z}\left(-\frac{11}{6} \mathrm{H}(0 ; z)-2 \mathrm{H}(0,0 ; z)-2 \mathrm{H}(0,1 ; z)\right. \\
& -2 \mathrm{H}(1,0 ; z))+\frac{1}{z}\left(-\frac{67}{18}+\frac{\pi^{2}}{2}-\frac{11}{6} \mathrm{H}(0 ; z)-\frac{11}{6} \mathrm{H}(1 ; z)-2 \mathrm{H}(0,0 ; z)-2 \mathrm{H}(0,1 ; z)\right. \\
& -2 \mathrm{H}(1,0 ; z)-2 \mathrm{H}(1,1 ; z))+\left(z-z^{2}\right)\left(\frac{\pi^{2}}{2}-2 \mathrm{H}(0,0 ; z)-2 \mathrm{H}(0,1 ; z)-2 \mathrm{H}(1,0 ; z)\right. \\
& -2 \mathrm{H}(1,1 ; z))] \\
& +\epsilon^{2}\left[6-\frac{28 \zeta_{3}}{3}+\delta(1-z)\left(\frac{1214}{81}-\frac{67 \pi^{2}}{72}-\frac{\pi^{4}}{48}-\frac{77 \zeta_{3}}{18}\right)+\mathcal{D}_{0}(z)\left(-\frac{202}{27}+\frac{11 \pi^{2}}{24}+\frac{14 \zeta_{3}}{3}\right)\right. \\
& +\left(\frac{67}{18}-\frac{\pi^{2}}{2}\right) \mathcal{D}_{1}(z)-\frac{11}{12} \mathcal{D}_{2}(z)+\frac{1}{3} \mathcal{D}_{3}(z)+2 \mathrm{H}(0 ; z)-\pi^{2} \mathrm{H}(0 ; z)+2 \mathrm{H}(1 ; z)-\pi^{2} \mathrm{H}(1 ; z) \\
& +4 \mathrm{H}(0,0,0 ; z)+4 \mathrm{H}(0,0,1 ; z)+4 \mathrm{H}(0,1,0 ; z)+4 \mathrm{H}(0,1,1 ; z)+4 \mathrm{H}(1,0,0 ; z) \\
& +4 \mathrm{H}(1,0,1 ; z)+4 \mathrm{H}(1,1,0 ; z)+4 \mathrm{H}(1,1,1 ; z)+\frac{1}{1-z}\left(-\frac{67}{18} \mathrm{H}(0 ; z)+\frac{1}{2} \pi^{2} \mathrm{H}(0 ; z)\right. \\
& -\frac{11}{6} \mathrm{H}(0,0 ; z)-\frac{11}{6} \mathrm{H}(0,1 ; z)-\frac{11}{6} \mathrm{H}(1,0 ; z)-2 \mathrm{H}(0,0,0 ; z)-2 \mathrm{H}(0,0,1 ; z) \\
& -2 \mathrm{H}(0,1,0 ; z)-2 \mathrm{H}(0,1,1 ; z)-2 \mathrm{H}(1,0,0 ; z)-2 \mathrm{H}(1,0,1 ; z)-2 \mathrm{H}(1,1,0 ; z)) \\
& +\frac{1}{z}\left(-\frac{202}{27}+\frac{11 \pi^{2}}{24}+\frac{14 \zeta_{3}}{3}-\frac{67}{18} \mathrm{H}(0 ; z)+\frac{1}{2} \pi^{2} \mathrm{H}(0 ; z)-\frac{67}{18} \mathrm{H}(1 ; z)+\frac{1}{2} \pi^{2} \mathrm{H}(1 ; z)\right. \\
& -\frac{11}{6} \mathrm{H}(0,0 ; z)-\frac{11}{6} \mathrm{H}(0,1 ; z)-\frac{11}{6} \mathrm{H}(1,0 ; z)-\frac{11}{6} \mathrm{H}(1,1 ; z)-2 \mathrm{H}(0,0,0 ; z) \\
& -2 \mathrm{H}(0,0,1 ; z)-2 \mathrm{H}(0,1,0 ; z)-2 \mathrm{H}(0,1,1 ; z)-2 \mathrm{H}(1,0,0 ; z)-2 \mathrm{H}(1,0,1 ; z) \\
& -2 \mathrm{H}(1,1,0 ; z)-2 \mathrm{H}(1,1,1 ; z))+\left(z-z^{2}\right)\left(+\frac{14 \zeta_{3}}{3}+\frac{1}{2} \pi^{2} \mathrm{H}(0 ; z)+\frac{1}{2} \pi^{2} \mathrm{H}(1 ; z)\right. \\
& -2 \mathrm{H}(0,0,0 ; z)-2 \mathrm{H}(0,0,1 ; z)-2 \mathrm{H}(0,1,0 ; z)-2 \mathrm{H}(0,1,1 ; z)-2 \mathrm{H}(1,0,0 ; z) \\
& -2 \mathrm{H}(1,0,1 ; z)-2 \mathrm{H}(1,1,0 ; z)-2 \mathrm{H}(1,1,1 ; z))] \\
& +\mathcal{O}\left(\epsilon^{3}\right)
\end{aligned}
$$

for the $(g, g g)$ case and to

$$
\begin{aligned}
& \mathcal{G}_{g, q \bar{q}}^{0}= \\
+ & \frac{1}{\epsilon}\left[-\frac{\delta(1-z)}{3}\right] \\
+ & {\left[-\frac{5}{9} \delta(1-z)+\frac{1}{3} \mathcal{D}_{0}(z)+\frac{1}{3 z}\right] } \\
+ & {\left[\delta(1-z)\left(-\frac{28}{27}+\frac{\pi^{2}}{12}\right)+\frac{5}{9} \mathcal{D}_{0}(z)-\frac{1}{3} \mathcal{D}_{1}(z)+\frac{1}{3(1-z)} \mathrm{H}(0 ; z)+\frac{1}{z}\left(\frac{5}{9}+\frac{1}{3} \mathrm{H}(0 ; z)+\frac{1}{3} \mathrm{H}(1 ; z)\right)\right] }
\end{aligned}
$$




$$
\begin{aligned}
+\epsilon^{2}[ & \delta(1-z)\left(-\frac{164}{81}+\frac{5 \pi^{2}}{36}+\frac{7 \zeta_{3}}{9}\right)+\left(\frac{28}{27}-\frac{\pi^{2}}{12}\right) \mathcal{D}_{0}(z)-\frac{5}{9} \mathcal{D}_{1}(z)+\frac{1}{6} \mathcal{D}_{2}(z)+\frac{1}{1-z}\left(\frac{5}{9} \mathrm{H}(0 ; z)\right. \\
& \left.+\frac{1}{3} \mathrm{H}(0,0 ; z)+\frac{1}{3} \mathrm{H}(0,1 ; z)+\frac{1}{3} \mathrm{H}(1,0 ; z)\right)+\frac{1}{z}\left(\frac{28}{27}-\frac{\pi^{2}}{12}+\frac{5}{9} \mathrm{H}(0 ; z)+\frac{5}{9} \mathrm{H}(1 ; z)\right. \\
& \left.\left.+\frac{1}{3} \mathrm{H}(0,0 ; z)+\frac{1}{3} \mathrm{H}(0,1 ; z)+\frac{1}{3} \mathrm{H}(1,0 ; z)+\frac{1}{3} \mathrm{H}(1,1 ; z)\right)\right] \\
+ & \mathcal{O}\left(\epsilon^{3}\right)
\end{aligned}
$$

for the $(g, q \bar{q})$ case.

The integrated form of the NNLO double real radiation antenna function for $(g, g g g)$ is:

$$
\begin{aligned}
& \mathcal{F}_{\text {g,ggg }}^{0}= \\
& +\frac{1}{\epsilon^{4}}[10 \delta(1-z)] \\
& +\frac{1}{\epsilon^{3}}\left[40+22 \delta(1-z)-20 \mathcal{D}_{0}(z)-\frac{20}{z}-20 z+20 z^{2}\right] \\
& +\frac{1}{\epsilon^{2}}\left[68+\delta(1-z)\left(\frac{590}{9}-8 \pi^{2}\right)-44 \mathcal{D}_{0}(z)+40 \mathcal{D}_{1}(z)+64 \mathrm{H}(0 ; z)+80 \mathrm{H}(1 ; z)-\frac{40}{1-z} \mathrm{H}(0 ; z)\right. \\
& -\frac{1}{z}\left(\frac{220}{3}+40 \mathrm{H}(0 ; z)+40 \mathrm{H}(1 ; z)\right)+z(-46-56 \mathrm{H}(0 ; z)-40 \mathrm{H}(1 ; z)) \\
& \left.+z^{2}\left(\frac{154}{3}+40 \mathrm{H}(0 ; z)+40 \mathrm{H}(1 ; z)\right)\right] \\
& +\frac{1}{\epsilon}\left[\frac{461}{3}-28 \pi^{2}+\delta(1-z)\left(\frac{4868}{27}-\frac{154 \pi^{2}}{9}-\frac{272 \zeta_{3}}{3}\right)+\left(-\frac{1180}{9}+16 \pi^{2}\right) \mathcal{D}_{0}(z)+88 \mathcal{D}_{1}(z)-40 \mathcal{D}_{2}(z)\right. \\
& +\frac{482}{3} \mathrm{H}(0 ; z)+136 \mathrm{H}(1 ; z)-16 \mathrm{H}(-1,0 ; z)+128 \mathrm{H}(0,0 ; z)+128 \mathrm{H}(0,1 ; z) \\
& +160 \mathrm{H}(1,0 ; z)+160 \mathrm{H}(1,1 ; z)+\frac{1}{1-z}(-88 \mathrm{H}(0 ; z)-84 \mathrm{H}(0,0 ; z)-80 \mathrm{H}(0,1 ; z) \\
& -80 \mathrm{H}(1,0 ; z))+\frac{1}{1+z}\left(\frac{2 \pi^{2}}{3}+8 \mathrm{H}(-1,0 ; z)-4 \mathrm{H}(0,0 ; z)\right)+\frac{1}{z}\left(-\frac{1556}{9}+\frac{46 \pi^{2}}{3}\right. \\
& -\frac{352}{3} \mathrm{H}(0 ; z)-\frac{440}{3} \mathrm{H}(1 ; z)-8 \mathrm{H}(-1,0 ; z)-80 \mathrm{H}(0,0 ; z)-80 \mathrm{H}(0,1 ; z)-80 \mathrm{H}(1,0 ; z) \\
& -80 \mathrm{H}(1,1 ; z))+z\left(-\frac{257}{3}+\frac{62 \pi^{2}}{3}-\frac{202}{3} \mathrm{H}(0 ; z)-92 \mathrm{H}(1 ; z)-8 \mathrm{H}(-1,0 ; z)\right. \\
& -128 \mathrm{H}(0,0 ; z)-112 \mathrm{H}(0,1 ; z)-80 \mathrm{H}(1,0 ; z)-80 \mathrm{H}(1,1 ; z))+z^{2}\left(\frac{778}{9}-\frac{50 \pi^{2}}{3}\right. \\
& +132 \mathrm{H}(0 ; z)+\frac{308}{3} \mathrm{H}(1 ; z)-8 \mathrm{H}(-1,0 ; z)+88 \mathrm{H}(0,0 ; z)+80 \mathrm{H}(0,1 ; z)+80 \mathrm{H}(1,0 ; z) \\
& +80 \mathrm{H}(1,1 ; z))] \\
& +\left[\frac{5249}{9}-\frac{592 \pi^{2}}{9}-\frac{1424 \zeta_{3}}{3}+\delta(1-z)\left(\frac{25811}{54}-\frac{1408 \pi^{2}}{27}+\frac{319 \pi^{4}}{180}-\frac{934 \zeta_{3}}{3}\right)+\mathcal{D}_{0}(z)\left(-\frac{9736}{27}\right.\right. \\
& \left.+\frac{308 \pi^{2}}{9}+\frac{544 \zeta_{3}}{3}\right)+\left(\frac{2360}{9}-32 \pi^{2}\right) \mathcal{D}_{1}(z)-88 \mathcal{D}_{2}(z)+\frac{80}{3} \mathcal{D}_{3}(z)+\frac{32}{3} \pi^{2} \mathrm{H}(-1 ; z)+\frac{1759}{9} \mathrm{H}(0 ; z) \\
& -64 \pi^{2} \mathrm{H}(0 ; z)+\frac{922}{3} \mathrm{H}(1 ; z)-\frac{200}{3} \pi^{2} \mathrm{H}(1 ; z)+16 \mathrm{H}(-1,0 ; z)+\frac{1030}{3} \mathrm{H}(0,0 ; z) \\
& +\frac{964}{3} \mathrm{H}(0,1 ; z)+\frac{808}{3} \mathrm{H}(1,0 ; z)+272 \mathrm{H}(1,1 ; z)+64 \mathrm{H}(-1,-1,0 ; z)-80 \mathrm{H}(-1,0,0 ; z)
\end{aligned}
$$




$$
\begin{aligned}
& -32 \mathrm{H}(-1,0,1 ; z)-96 \mathrm{H}(0,-1,0 ; z)+256 \mathrm{H}(0,0,0 ; z)+256 \mathrm{H}(0,0,1 ; z) \\
& +224 \mathrm{H}(0,1,0 ; z)+256 \mathrm{H}(0,1,1 ; z)+272 \mathrm{H}(1,0,0 ; z)+320 \mathrm{H}(1,0,1 ; z)+304 \mathrm{H}(1,1,0 ; z) \\
& +320 \mathrm{H}(1,1,1 ; z)+\frac{1}{1-z}\left(-\frac{22 \pi^{2}}{9}+44 \zeta_{3}-\frac{2360}{9} \mathrm{H}(0 ; z)+40 \pi^{2} \mathrm{H}(0 ; z)+\frac{4}{3} \pi^{2} \mathrm{H}(1 ; z)\right. \\
& -\frac{616}{3} \mathrm{H}(0,0 ; z)-176 \mathrm{H}(0,1 ; z)-\frac{572}{3} \mathrm{H}(1,0 ; z)+24 \mathrm{H}(0,-1,0 ; z)-172 \mathrm{H}(0,0,0 ; z) \\
& -168 \mathrm{H}(0,0,1 ; z)-144 \mathrm{H}(0,1,0 ; z)-160 \mathrm{H}(0,1,1 ; z)-136 \mathrm{H}(1,0,0 ; z)-160 \mathrm{H}(1,0,1 ; z) \\
& -152 \mathrm{H}(1,1,0 ; z))+\frac{1}{1+z}\left(+28 \zeta_{3}-\frac{16}{3} \pi^{2} \mathrm{H}(-1 ; z)+\frac{4}{3} \pi^{2} \mathrm{H}(0 ; z)-32 \mathrm{H}(-1,-1,0 ; z)\right. \\
& +40 \mathrm{H}(-1,0,0 ; z)+16 \mathrm{H}(-1,0,1 ; z)+24 \mathrm{H}(0,-1,0 ; z)-12 \mathrm{H}(0,0,0 ; z)-8 \mathrm{H}(0,0,1 ; z)) \\
& +\frac{1}{z}\left(-\frac{2102}{3}+\frac{550 \pi^{2}}{9}+\frac{592 \zeta_{3}}{3}+\frac{16}{3} \pi^{2} \mathrm{H}(-1 ; z)-\frac{1216}{3} \mathrm{H}(0 ; z)+\frac{116}{3} \pi^{2} \mathrm{H}(0 ; z)\right. \\
& -\frac{3112}{9} \mathrm{H}(1 ; z)+\frac{100}{3} \pi^{2} \mathrm{H}(1 ; z)+\frac{88}{3} \mathrm{H}(-1,0 ; z)-\frac{704}{3} \mathrm{H}(0,0 ; z)-\frac{704}{3} \mathrm{H}(0,1 ; z) \\
& -\frac{836}{3} \mathrm{H}(1,0 ; z)-\frac{880}{3} \mathrm{H}(1,1 ; z)+32 \mathrm{H}(-1,-1,0 ; z)-40 \mathrm{H}(-1,0,0 ; z)-16 \mathrm{H}(-1,0,1 ; z) \\
& -160 \mathrm{H}(0,0,0 ; z)-160 \mathrm{H}(0,0,1 ; z)-144 \mathrm{H}(0,1,0 ; z)-160 \mathrm{H}(0,1,1 ; z)-136 \mathrm{H}(1,0,0 ; z) \\
& -160 \mathrm{H}(1,0,1 ; z)-152 \mathrm{H}(1,1,0 ; z)-160 \mathrm{H}(1,1,1 ; z))+z\left(-\frac{3305}{9}+\frac{311 \pi^{2}}{9}+\frac{688 \zeta_{3}}{3}\right. \\
& +\frac{16}{3} \pi^{2} \mathrm{H}(-1 ; z)-\frac{2363}{9} \mathrm{H}(0 ; z)+\frac{172}{3} \pi^{2} \mathrm{H}(0 ; z)-\frac{514}{3} \mathrm{H}(1 ; z)+\frac{100}{3} \pi^{2} \mathrm{H}(1 ; z) \\
& +16 \mathrm{H}(-1,0 ; z)-\frac{362}{3} \mathrm{H}(0,0 ; z)-\frac{404}{3} \mathrm{H}(0,1 ; z)-\frac{536}{3} \mathrm{H}(1,0 ; z)-184 \mathrm{H}(1,1 ; z) \\
& +32 \mathrm{H}(-1,-1,0 ; z)-40 \mathrm{H}(-1,0,0 ; z)-16 \mathrm{H}(-1,0,1 ; z)-272 \mathrm{H}(0,0,0 ; z) \\
& -256 \mathrm{H}(0,0,1 ; z)-208 \mathrm{H}(0,1,0 ; z)-224 \mathrm{H}(0,1,1 ; z)-136 \mathrm{H}(1,0,0 ; z)-160 \mathrm{H}(1,0,1 ; z) \\
& -152 \mathrm{H}(1,1,0 ; z)-160 \mathrm{H}(1,1,1 ; z))+z^{2}\left(\frac{10382}{27}-\frac{473 \pi^{2}}{9}-\frac{760 \zeta_{3}}{3}+\frac{16}{3} \pi^{2} \mathrm{H}(-1 ; z)\right. \\
& +\frac{340}{3} \mathrm{H}(0 ; z)-\frac{124}{3} \pi^{2} \mathrm{H}(0 ; z)+\frac{1556}{9} \mathrm{H}(1 ; z)-\frac{100}{3} \pi^{2} \mathrm{H}(1 ; z)+\frac{88}{3} \mathrm{H}(-1,0 ; z) \\
& +\frac{880}{3} \mathrm{H}(0,0 ; z)+264 \mathrm{H}(0,1 ; z)+\frac{616}{3} \mathrm{H}(1,0 ; z)+\frac{616}{3} \mathrm{H}(1,1 ; z) \\
& +32 \mathrm{H}(-1,-1,0 ; z)-40 \mathrm{H}(-1,0,0 ; z)-16 \mathrm{H}(-1,0,1 ; z)-48 \mathrm{H}(0,-1,0 ; z) \\
& +184 \mathrm{H}(0,0,0 ; z)+176 \mathrm{H}(0,0,1 ; z)+144 \mathrm{H}(0,1,0 ; z)+160 \mathrm{H}(0,1,1 ; z)+136 \mathrm{H}(1,0,0 ; z) \\
& +160 \mathrm{H}(1,0,1 ; z)+152 \mathrm{H}(1,1,0 ; z)+160 \mathrm{H}(1,1,1 ; z))] \\
& +\mathcal{O}(\epsilon)
\end{aligned}
$$

The $(g, q \bar{q} g)$ antenna function has a leading and subleading colour contribution. They integrate to:

$$
\begin{aligned}
& \mathcal{G}_{g, q \bar{q} g}^{0}= \\
+ & \frac{1}{\epsilon^{3}}\left[-\frac{5}{3} \delta(1-z)\right] \\
+ & \frac{1}{\epsilon^{2}}\left[-\frac{7}{2}-\frac{47}{9} \delta(1-z)+\frac{10}{3} \mathcal{D}_{0}(z)+\mathrm{H}(0 ; z)+\frac{4}{z}+z\left(\frac{3}{2}+\mathrm{H}(0 ; z)\right)-\frac{8}{3} z^{2}\right]
\end{aligned}
$$




$$
\begin{aligned}
+\frac{1}{\epsilon}[ & -\frac{59}{6}-\frac{\pi^{2}}{3}+\delta(1-z)\left(-\frac{557}{36}+\frac{23 \pi^{2}}{18}\right)+\frac{94}{9} \mathcal{D}_{0}(z)-\frac{20}{3} \mathcal{D}_{1}(z)-\frac{35}{6} \mathrm{H}(0 ; z)-7 \mathrm{H}(1 ; z) \\
& +\frac{20 \mathrm{H}(0 ; z)}{3(1-z)}+\frac{1}{z}\left(\frac{148}{9}+8 \mathrm{H}(0 ; z)+8 \mathrm{H}(1 ; z)\right)+3 \mathrm{H}(0,0 ; z)+2 \mathrm{H}(0,1 ; z)+z\left(\frac{41}{6}-\frac{\pi^{2}}{3}\right. \\
& \left.\left.+\frac{25}{6} \mathrm{H}(0 ; z)+3 \mathrm{H}(1 ; z)+3 \mathrm{H}(0,0 ; z)+2 \mathrm{H}(0,1 ; z)\right)+z^{2}\left(-\frac{28}{3}-\frac{16}{3} \mathrm{H}(0 ; z)-\frac{16}{3} \mathrm{H}(1 ; z)\right)\right] \\
+[- & \frac{259}{12}+\frac{11 \pi^{2}}{4}-2 \zeta_{3}+\delta(1-z)\left(-\frac{28613}{648}+\frac{25 \pi^{2}}{6}+\frac{118 \zeta_{3}}{9}\right)+\left(\frac{557}{18}-\frac{23 \pi^{2}}{9}\right) \mathcal{D}_{0}(z)-\frac{188}{9} \mathcal{D}_{1}(z) \\
& +\frac{20}{3} \mathcal{D}_{2}(z)-\frac{113}{9} \mathrm{H}(0 ; z)-\frac{7}{6} \pi^{2} \mathrm{H}(0 ; z)-\frac{59}{3} \mathrm{H}(1 ; z)-\frac{59}{6} \mathrm{H}(0,0 ; z)-\frac{35}{3} \mathrm{H}(0,1 ; z) \\
& -\frac{40}{3} \mathrm{H}(1,0 ; z)-14 \mathrm{H}(1,1 ; z)+\frac{1}{1-z}\left(\frac{2 \pi^{2}}{9}+\frac{188}{9} \mathrm{H}(0 ; z)+\frac{44}{3} \mathrm{H}(0,0 ; z)+\frac{40}{3} \mathrm{H}(0,1 ; z)\right. \\
& \left.+\frac{44}{3} \mathrm{H}(1,0 ; z)\right)+\frac{1}{z}\left(\frac{3065}{54}-\frac{10 \pi^{2}}{3}+\frac{296}{9} \mathrm{H}(0 ; z)+\frac{296}{9} \mathrm{H}(1 ; z)+16 \mathrm{H}(0,0 ; z)\right. \\
& +16 \mathrm{H}(0,1 ; z)+16 \mathrm{H}(1,0 ; z)+16 \mathrm{H}(1,1 ; z))+7 \mathrm{H}(0,0,0 ; z)+6 \mathrm{H}(0,0,1 ; z) \\
& +4 \mathrm{H}(0,1,0 ; z)+4 \mathrm{H}(0,1,1 ; z)+z\left(\frac{71}{4}-\frac{25 \pi^{2}}{12}-2 \zeta_{3}+\frac{160}{9} \mathrm{H}(0 ; z)-\frac{7}{6} \pi^{2} \mathrm{H}(0 ; z)\right. \\
& +\frac{41}{3} \mathrm{H}(1 ; z)+\frac{49}{6} \mathrm{H}(0,0 ; z)+\frac{25}{3} \mathrm{H}(0,1 ; z)+\frac{14}{3} \mathrm{H}(1,0 ; z)+6 \mathrm{H}(1,1 ; z)+7 \mathrm{H}(0,0,0 ; z) \\
& +6 \mathrm{H}(0,0,1 ; z)+4 \mathrm{H}(0,1,0 ; z)+4 \mathrm{H}(0,1,1 ; z))+z^{2}\left(-\frac{757}{27}+\frac{20 \pi^{2}}{9}-\frac{56}{3} \mathrm{H}(0 ; z)\right. \\
& \left.\left.+\frac{56}{3} \mathrm{H}(1 ; z)-\frac{32}{3} \mathrm{H}(0,0 ; z)-\frac{32}{3} \mathrm{H}(0,1 ; z)-\frac{32}{3} \mathrm{H}(1,0 ; z)-\frac{32}{3} \mathrm{H}(1,1 ; z)\right)\right] \\
+ & \mathcal{O}(\epsilon)
\end{aligned}
$$

and

$$
\begin{aligned}
& \tilde{\mathcal{G}}_{g, q q \bar{q} g}^{0}= \\
& +\frac{1}{\epsilon^{3}}\left[-\frac{2}{3} \delta(1-z)\right] \\
& +\frac{1}{\epsilon^{2}}\left[1-\frac{19}{9} \delta(1-z)+\frac{4}{3} \mathcal{D}_{0}(z)+2 \mathrm{H}(0 ; z)+\frac{8}{3 z}+z(-1+2 \mathrm{H}(0 ; z))-\frac{4}{3} z^{2}\right] \\
& +\frac{1}{\epsilon}\left[\frac{7}{3}-\frac{2 \pi^{2}}{3}+\delta(1-z)\left(-\frac{373}{54}+\frac{5 \pi^{2}}{9}\right)+\frac{38}{9} \mathcal{D}_{0}(z)-\frac{8}{3} \mathcal{D}_{1}(z)+3 \mathrm{H}(0 ; z)+2 \mathrm{H}(1 ; z)+6 \mathrm{H}(0,0 ; z)\right. \\
& \quad+4 \mathrm{H}(0,1 ; z)+\frac{8 \mathrm{H}(0 ; z)}{3(1-z)}+\frac{1}{z}\left(\frac{76}{9}+\frac{16}{3} \mathrm{H}(0 ; z)+\frac{16}{3} \mathrm{H}(1 ; z)\right)+z\left(-\frac{7}{3}-\frac{2 \pi^{2}}{3}-\mathrm{H}(0 ; z)\right. \\
& \left.\quad-2 \mathrm{H}(1 ; z)+6 \mathrm{H}(0,0 ; z)+4 \mathrm{H}(0,1 ; z))+z^{2}\left(-\frac{38}{9}-\frac{8}{3} \mathrm{H}(0 ; z)-\frac{8}{3} \mathrm{H}(1 ; z)\right)\right] \\
& +\left[\frac{391}{18}-\frac{13 \pi^{2}}{18}-4 \zeta_{3}+\delta(1-z)\left(-\frac{6973}{324}+\frac{95 \pi^{2}}{54}+\frac{64 \zeta_{3}}{9}\right)+\left(\frac{373}{27}-\frac{10 \pi^{2}}{9}\right) \mathcal{D}_{0}(z)-\frac{76}{9} \mathcal{D}_{1}(z)\right. \\
& \quad+\frac{8}{3} \mathcal{D}_{2}(z)+\frac{52}{3} \mathrm{H}(0 ; z)-\frac{7}{3} \pi^{2} \mathrm{H}(0 ; z)+\frac{14}{3} \mathrm{H}(1 ; z)+\frac{25}{3} \mathrm{H}(0,0 ; z)+6 \mathrm{H}(0,1 ; z) \\
& \quad+\frac{16}{3} \mathrm{H}(1,0 ; z)+4 \mathrm{H}(1,1 ; z)+14 \mathrm{H}(0,0,0 ; z)+12 \mathrm{H}(0,0,1 ; z)+8 \mathrm{H}(0,1,0 ; z) \\
& \quad+8 \mathrm{H}(0,1,1 ; z)+\frac{1}{1-z}\left(\frac{76}{9} \mathrm{H}(0 ; z)+8 \mathrm{H}(0,0 ; z)+\frac{16}{3} \mathrm{H}(0,1 ; z)+\frac{16}{3} \mathrm{H}(1,0 ; z)\right)
\end{aligned}
$$




$$
\begin{aligned}
& +\frac{1}{z}\left(\frac{809}{27}-\frac{28 \pi^{2}}{9}+\frac{152}{9} \mathrm{H}(0 ; z)+\frac{152}{9} \mathrm{H}(1 ; z)+\frac{32}{3} \mathrm{H}(0,0 ; z)\right. \\
& \left.+\frac{32}{3} \mathrm{H}(0,1 ; z)+8 \mathrm{H}(1,0 ; z)+\frac{32}{3} \mathrm{H}(1,1 ; z)\right)+z\left(-\frac{325}{18}-\frac{7 \pi^{2}}{18}-4 \zeta_{3}+\frac{4}{3} \mathrm{H}(0 ; z)\right. \\
& -\frac{7}{3} \pi^{2} \mathrm{H}(0 ; z)-\frac{14}{3} \mathrm{H}(1 ; z)-\frac{11}{3} \mathrm{H}(0,0 ; z)-2 \mathrm{H}(0,1 ; z)-\frac{20}{3} \mathrm{H}(1,0 ; z)-4 \mathrm{H}(1,1 ; z) \\
& +14 \mathrm{H}(0,0,0 ; z)+12 \mathrm{H}(0,0,1 ; z)+8 \mathrm{H}(0,1,0 ; z)+8 \mathrm{H}(0,1,1 ; z))+z^{2}\left(-\frac{346}{27}+\frac{10 \pi^{2}}{9}\right. \\
& \left.\left.-\frac{76}{9} \mathrm{H}(0 ; z)-\frac{76}{9} \mathrm{H}(1 ; z)-\frac{16}{3} \mathrm{H}(0,0 ; z)-\frac{16}{3} \mathrm{H}(0,1 ; z)-\frac{16}{3} \mathrm{H}(1,0 ; z)-\frac{16}{3} \mathrm{H}(1,1 ; z)\right)\right] \\
& +\mathcal{O}(\epsilon) .
\end{aligned}
$$

Finally, the integrated one-loop antenna functions for $(g, g g)$ at leading colour and for a closed quark loop read:

$$
\begin{aligned}
& \mathcal{F}_{g, g g}^{1}= \\
& +\frac{1}{\epsilon^{4}}\left[-\frac{\delta(1-z)}{2}\right] \\
& +\frac{1}{\epsilon^{3}}\left[-2-\frac{55}{12} \delta(1-z)+\mathcal{D}_{0}(z)+\frac{1}{z}+z-z^{2}\right] \\
& +\frac{1}{\epsilon^{2}}\left[-\frac{22}{3}+\delta(1-z)\left(-\frac{85}{12}+\frac{7 \pi^{2}}{12}\right)+\frac{11}{2} \mathcal{D}_{0}(z)-2 \mathcal{D}_{1}(z)-8 \mathrm{H}(0 ; z)-4 \mathrm{H}(1 ; z)+\frac{4 \mathrm{H}(0 ; z)}{1-z}\right. \\
& +\frac{1}{z}\left(\frac{11}{2}+4 \mathrm{H}(0 ; z)+2 \mathrm{H}(1 ; z)\right)+z\left(\frac{11}{3}+4 \mathrm{H}(0 ; z)+2 \mathrm{H}(1 ; z)\right) \\
& \left.+z^{2}\left(-\frac{11}{3}-4 \mathrm{H}(0 ; z)-2 \mathrm{H}(1 ; z)\right)\right] \\
& +\frac{1}{\epsilon}\left[-2+\frac{7 \pi^{2}}{3}+\delta(1-z)\left(-\frac{1967}{108}+\frac{385 \pi^{2}}{72}+\frac{22 \zeta_{3}}{3}\right)+\left(\frac{389}{36}-\frac{7 \pi^{2}}{6}\right) \mathcal{D}_{0}(z)-\frac{22}{3} \mathcal{D}_{1}(z)+2 \mathcal{D}_{2}(z)\right. \\
& -\frac{22}{3} \mathrm{H}(0 ; z)-\frac{22}{3} \mathrm{H}(1 ; z)-24 \mathrm{H}(0,0 ; z)-12 \mathrm{H}(0,1 ; z)-12 \mathrm{H}(1,0 ; z)-8 \mathrm{H}(1,1 ; z) \\
& +\frac{1}{1-z}(11 \mathrm{H}(0 ; z)+12 \mathrm{H}(0,0 ; z)+6 \mathrm{H}(0,1 ; z)+6 \mathrm{H}(1,0 ; z))+z\left(\frac{1}{6}-\frac{7 \pi^{2}}{6}+\frac{11}{3} \mathrm{H}(0 ; z)\right. \\
& \left.+\frac{11}{3} \mathrm{H}(1 ; z)+12 \mathrm{H}(0,0 ; z)+6 \mathrm{H}(0,1 ; z)+6 \mathrm{H}(1,0 ; z)+4 \mathrm{H}(1,1 ; z)\right)+z^{2}\left(\frac{7 \pi^{2}}{6}\right. \\
& \left.-\frac{11}{3} \mathrm{H}(0 ; z)-\frac{11}{3} \mathrm{H}(1 ; z)-12 \mathrm{H}(0,0 ; z)-6 \mathrm{H}(0,1 ; z)-6 \mathrm{H}(1,0 ; z)-4 \mathrm{H}(1,1 ; z)\right) \\
& +\frac{1}{z}\left(\frac{389}{36}-\frac{7 \pi^{2}}{6}+11 \mathrm{H}(0 ; z)+\frac{22}{3} \mathrm{H}(1 ; z)+12 \mathrm{H}(0,0 ; z)+6 \mathrm{H}(0,1 ; z)+6 \mathrm{H}(1,0 ; z)\right. \\
& +4 \mathrm{H}(1,1 ; z))] \\
& +\left[-13+\frac{169 \pi^{2}}{18}+\frac{100 \zeta_{3}}{3}+\delta(1-z)\left(-\frac{14453}{324}+\frac{3155 \pi^{2}}{432}-\frac{11 \pi^{4}}{72}+\frac{269 \zeta_{3}}{6}\right)+\left(\frac{3197}{108}-\frac{55 \pi^{2}}{9}\right.\right. \\
& \left.-\frac{44 \zeta_{3}}{3}\right) \mathcal{D}_{0}(z)+\left(-\frac{73}{4}+\frac{7 \pi^{2}}{3}\right) \mathcal{D}_{1}(z)+\frac{11}{2} \mathcal{D}_{2}(z)-\frac{4}{3} \mathcal{D}_{3}(z)-\frac{20}{3} \mathrm{H}(0 ; z)+\frac{28}{3} \pi^{2} \mathrm{H}(0 ; z)-4 \mathrm{H}(1 ; z) \\
& +\frac{16}{3} \pi^{2} \mathrm{H}(1 ; z)-\frac{20}{3} \mathrm{H}(0,0 ; z)-\frac{22}{3} \mathrm{H}(0,1 ; z)-\frac{20}{3} \mathrm{H}(1,0 ; z)-\frac{22}{3} \mathrm{H}(1,1 ; z)
\end{aligned}
$$




$$
\begin{aligned}
& -56 \mathrm{H}(0,0,0 ; z)-32 \mathrm{H}(0,0,1 ; z)-24 \mathrm{H}(0,1,0 ; z)-20 \mathrm{H}(0,1,1 ; z)-24 \mathrm{H}(1,0,0 ; z) \\
& -20 \mathrm{H}(1,0,1 ; z)-16 \mathrm{H}(1,1,0 ; z)-16 \mathrm{H}(1,1,1 ; z)+\frac{1}{1-z}\left(\frac{11 \pi^{2}}{18}-2 \zeta_{3}+\frac{925}{36} \mathrm{H}(0 ; z)\right. \\
& -\frac{14}{3} \pi^{2} \mathrm{H}(0 ; z)-\frac{1}{3} \pi^{2} \mathrm{H}(1 ; z)+\frac{88}{3} \mathrm{H}(0,0 ; z)+\frac{44}{3} \mathrm{H}(0,1 ; z)+\frac{55}{3} \mathrm{H}(1,0 ; z) \\
& +28 \mathrm{H}(0,0,0 ; z)+16 \mathrm{H}(0,0,1 ; z)+12 \mathrm{H}(0,1,0 ; z)+10 \mathrm{H}(0,1,1 ; z)+12 \mathrm{H}(1,0,0 ; z) \\
& +10 \mathrm{H}(1,0,1 ; z)+8 \mathrm{H}(1,1,0 ; z))+\frac{1}{z}\left(\frac{3179}{108}-\frac{143 \pi^{2}}{18}-\frac{50 \zeta_{3}}{3}+\frac{925}{36} \mathrm{H}(0 ; z)\right. \\
& -\frac{14}{3} \pi^{2} \mathrm{H}(0 ; z)+\frac{73}{4} \mathrm{H}(1 ; z)-\frac{8}{3} \pi^{2} \mathrm{H}(1 ; z)+22 \mathrm{H}(0,0 ; z)+\frac{44}{3} \mathrm{H}(0,1 ; z)+11 \mathrm{H}(1,0 ; z) \\
& +11 \mathrm{H}(1,1 ; z)+28 \mathrm{H}(0,0,0 ; z)+16 \mathrm{H}(0,0,1 ; z)+12 \mathrm{H}(0,1,0 ; z)+10 \mathrm{H}(0,1,1 ; z) \\
& +12 \mathrm{H}(1,0,0 ; z)+10 \mathrm{H}(1,0,1 ; z)+8 \mathrm{H}(1,1,0 ; z)+8 \mathrm{H}(1,1,1 ; z))+z\left(\frac{11}{18}-\frac{181 \pi^{2}}{36}\right. \\
& -\frac{50 \zeta_{3}}{3}+\frac{1}{3} \mathrm{H}(0 ; z)-\frac{14}{3} \pi^{2} \mathrm{H}(0 ; z)+\frac{1}{6} \mathrm{H}(1 ; z)-\frac{8}{3} \pi^{2} \mathrm{H}(1 ; z)+\frac{7}{3} \mathrm{H}(0,0 ; z)+\frac{11}{3} \mathrm{H}(0,1 ; z) \\
& +\frac{7}{3} \mathrm{H}(1,0 ; z)+\frac{11}{3} \mathrm{H}(1,1 ; z)+28 \mathrm{H}(0,0,0 ; z)+16 \mathrm{H}(0,0,1 ; z)+12 \mathrm{H}(0,1,0 ; z) \\
& +10 \mathrm{H}(0,1,1 ; z)+12 \mathrm{H}(1,0,0 ; z)+10 \mathrm{H}(1,0,1 ; z)+8 \mathrm{H}(1,1,0 ; z)+8 \mathrm{H}(1,1,1 ; z)) \\
& +z^{2}\left(\frac{55 \pi^{2}}{12}+\frac{50 \zeta_{3}}{3}+\frac{14}{3} \pi^{2} \mathrm{H}(0 ; z)+\frac{8}{3} \pi^{2} \mathrm{H}(1 ; z)-\frac{11}{3} \mathrm{H}(0,0 ; z)-\frac{11}{3} \mathrm{H}(0,1 ; z)\right. \\
& +\frac{11}{3} \mathrm{H}(1,0 ; z)-\frac{11}{3} \mathrm{H}(1,1 ; z)-28 \mathrm{H}(0,0,0 ; z)-16 \mathrm{H}(0,0,1 ; z)-12 \mathrm{H}(0,1,0 ; z) \\
& -10 \mathrm{H}(0,1,1 ; z)-12 \mathrm{H}(1,0,0 ; z)-10 \mathrm{H}(1,0,1 ; z)-8 \mathrm{H}(1,1,0 ; z)-8 \mathrm{H}(1,1,1 ; z))] \\
& +\mathcal{O}(\epsilon)
\end{aligned}
$$

and

$$
\begin{aligned}
& \hat{\mathcal{F}}_{g, g g}^{1}= \\
& +\frac{1}{\epsilon^{3}}\left[\frac{2}{3} \delta(1-z)\right] \\
& +\frac{1}{\epsilon^{2}}\left[\frac{4}{3}+\frac{11}{18} \delta(1-z)-\frac{2}{3} \mathcal{D}_{0}(z)-\frac{2}{3 z}-\frac{2}{3} z+\frac{2}{3} z^{2}\right] \\
& +\frac{1}{\epsilon}\left[\delta(1-z)\left(\frac{143}{108}-\frac{5 \pi^{2}}{6}\right)-\frac{11}{18} \mathcal{D}_{0}(z)+\frac{2}{3} \mathcal{D}_{1}(z)+\frac{4}{3} \mathrm{H}(0 ; z)+\frac{4}{3} \mathrm{H}(1 ; z)-\frac{2 \mathrm{H}(0 ; z)}{3(1-z)}-\frac{1}{z}\left(\frac{11}{18}\right.\right. \\
& \left.\left.\quad+\frac{2}{3} \mathrm{H}(0 ; z)+\frac{2}{3} \mathrm{H}(1 ; z)\right)-z\left(\frac{1}{6}+\frac{2}{3} \mathrm{H}(0 ; z)+\frac{2}{3} \mathrm{H}(1 ; z)\right)+z^{2}\left(\frac{2}{3} \mathrm{H}(0 ; z)+\frac{2}{3} \mathrm{H}(1 ; z)\right)\right] \\
& +\left[\frac{4}{3}-\frac{5 \pi^{2}}{3}+\delta(1-z)\left(\frac{979}{324}-\frac{55 \pi^{2}}{72}-\frac{14 \zeta_{3}}{9}\right)+\left(-\frac{38}{27}+\frac{5 \pi^{2}}{6}\right) \mathcal{D}_{0}(z)+\frac{11}{18} \mathcal{D}_{1}(z)-\frac{1}{3} \mathcal{D}_{2}(z)\right. \\
& \quad+\frac{4}{3} \mathrm{H}(0,0 ; z)+\frac{4}{3} \mathrm{H}(0,1 ; z)+\frac{4}{3} \mathrm{H}(1,0 ; z)+\frac{4}{3} \mathrm{H}(1,1 ; z)+\frac{1}{1-z}\left(-\frac{11}{18} \mathrm{H}(0 ; z)\right. \\
& \left.\quad-\frac{2}{3} \mathrm{H}(0,0 ; z)-\frac{2}{3} \mathrm{H}(0,1 ; z)-\frac{2}{3} \mathrm{H}(1,0 ; z)\right)+\frac{1}{z}\left(-\frac{85}{54}+\frac{5 \pi^{2}}{6}-\frac{11}{18} \mathrm{H}(0 ; z)-\frac{11}{18} \mathrm{H}(1 ; z)\right. \\
& \left.\quad-\frac{2}{3} \mathrm{H}(0,0 ; z)-\frac{2}{3} \mathrm{H}(0,1 ; z)-\frac{2}{3} \mathrm{H}(1,0 ; z)-\frac{2}{3} \mathrm{H}(1,1 ; z)\right)+z\left(-\frac{7}{9}+\frac{5 \pi^{2}}{6}-\frac{1}{3} \mathrm{H}(0 ; z)\right.
\end{aligned}
$$




$$
\begin{aligned}
& \left.\quad-\frac{1}{6} \mathrm{H}(1 ; z)-\frac{2}{3} \mathrm{H}(0,0 ; z)-\frac{2}{3} \mathrm{H}(0,1 ; z)-\frac{2}{3} \mathrm{H}(1,0 ; z)-\frac{2}{3} \mathrm{H}(1,1 ; z)\right)+z^{2}\left(-\frac{5 \pi^{2}}{6}\right. \\
& \left.\left.+\frac{2}{3} \mathrm{H}(0,0 ; z)+\frac{2}{3} \mathrm{H}(0,1 ; z)+\frac{2}{3} \mathrm{H}(1,0 ; z)+\frac{2}{3} \mathrm{H}(1,1 ; z)\right)\right] \\
& +\mathcal{O}(\epsilon)
\end{aligned}
$$

while the leading and subleading colour and quark loop contributions to the one-loop $(g, q \bar{q})$ antenna function integrate to:

$$
\begin{aligned}
& \mathcal{G}_{g, q \bar{q}}^{1}= \\
& +\frac{1}{\epsilon^{2}}\left[\frac{11}{18} \delta(1-z)\right] \\
& +\frac{1}{\epsilon}\left[\frac{119}{108} \delta(1-z)-\frac{11}{18} \mathcal{D}_{0}(z)-\frac{2 \mathrm{H}(0 ; z)}{3(1-z)}+\frac{1}{z}\left(-\frac{11}{18}-\frac{2}{3} \mathrm{H}(0 ; z)\right)\right] \\
& +\left[-\frac{2}{3}-\frac{\pi^{2}}{9}+\delta(1-z)\left(\frac{787}{324}-\frac{55 \pi^{2}}{72}\right)-\frac{32}{27} \mathcal{D}_{0}(z)+\frac{11}{18} \mathcal{D}_{1}(z)-\frac{2}{3} \mathrm{H}(0 ; z)-\frac{1}{3} \mathrm{H}(0,0 ; z)\right. \\
& \quad-\frac{1}{3} \mathrm{H}(1,0 ; z)+\frac{1}{1-z}\left(-\frac{\pi^{2}}{9}-\frac{31}{18} \mathrm{H}(0 ; z)-\frac{8}{3} \mathrm{H}(0,0 ; z)-\frac{2}{3} \mathrm{H}(0,1 ; z)-\frac{4}{3} \mathrm{H}(1,0 ; z)\right) \\
& \quad+\frac{1}{z}\left(-\frac{83}{108}+\frac{\pi^{2}}{9}-\frac{31}{18} \mathrm{H}(0 ; z)-\frac{11}{18} \mathrm{H}(1 ; z)-2 \mathrm{H}(0,0 ; z)-\frac{2}{3} \mathrm{H}(0,1 ; z)-\frac{2}{3} \mathrm{H}(1,0 ; z)\right) \\
& \left.\quad+z\left(\frac{2 \pi^{2}}{9}+\frac{2}{3} \mathrm{H}(0,0 ; z)+\frac{2}{3} \mathrm{H}(1,0 ; z)\right)\right] \\
& +\mathcal{O}(\epsilon)
\end{aligned}
$$

and

$$
\begin{aligned}
& \tilde{\mathcal{G}}_{g, q \bar{q}}^{1}= \\
& +\frac{1}{\epsilon^{3}}\left[\frac{1}{6} \delta(1-z)\right] \\
& +\frac{1}{\epsilon^{2}}\left[\frac{19}{36} \delta(1-z)-\frac{1}{3} \mathcal{D}_{0}(z)-\frac{1}{3 z}\right] \\
& +\frac{1}{\epsilon}\left[\delta(1-z)\left(\frac{173}{108}-\frac{5 \pi^{2}}{36}\right)-\frac{19}{18} \mathcal{D}_{0}(z)+\frac{2}{3} \mathcal{D}_{1}(z)-\frac{2 \mathrm{H}(0 ; z)}{3(1-z)}+\frac{1}{z}\left(-\frac{19}{18}-\frac{2}{3} \mathrm{H}(0 ; z)-\frac{2}{3} \mathrm{H}(1 ; z)\right)\right] \\
& +\left[-\frac{2}{3}-\frac{\pi^{2}}{9}+\delta(1-z)\left(\frac{343}{81}-\frac{95 \pi^{2}}{216}-\frac{7 \zeta_{3}}{9}\right)+\left(-\frac{173}{54}+\frac{5 \pi^{2}}{18}\right) \mathcal{D}_{0}(z)+\frac{19}{9} \mathcal{D}_{1}(z)-\frac{2}{3} \mathcal{D}_{2}(z)\right. \\
& \quad-\frac{2}{3} \mathrm{H}(0 ; z)-\frac{1}{3} \mathrm{H}(0,0 ; z)-\frac{1}{3} \mathrm{H}(1,0 ; z)+\frac{1}{1-z}\left(-\frac{19}{9} \mathrm{H}(0 ; z)-2 \mathrm{H}(0,0 ; z)-\frac{4}{3} \mathrm{H}(0,1 ; z)\right. \\
& \left.\quad-\frac{4}{3} \mathrm{H}(1,0 ; z)\right)+\frac{1}{z}\left(-\frac{355}{108}+\frac{\pi^{2}}{2}-\frac{19}{9} \mathrm{H}(0 ; z)-\frac{19}{9} \mathrm{H}(1 ; z)-\frac{4}{3} \mathrm{H}(0,0 ; z)-\frac{4}{3} \mathrm{H}(0,1 ; z)\right. \\
& \left.\left.\quad-\frac{2}{3} \mathrm{H}(1,0 ; z)-\frac{4}{3} \mathrm{H}(1,1 ; z)\right)+z\left(\frac{2 \pi^{2}}{9}+\frac{2}{3} \mathrm{H}(0,0 ; z)+\frac{2}{3} \mathrm{H}(1,0 ; z)\right)\right] \\
& +\mathcal{O}(\epsilon)
\end{aligned}
$$

and

$$
\begin{aligned}
\hat{\mathcal{G}}_{g, q \bar{q}}^{1}= \\
+\frac{1}{\epsilon}\left[\frac{5}{27} \delta(1-z)-\frac{1}{9} \mathcal{D}_{0}(z)-\frac{1}{9 z}\right]
\end{aligned}
$$




$$
\begin{aligned}
& +\left[\delta(1-z)\left(\frac{53}{81}+\frac{5 \pi^{2}}{108}\right)-\frac{5}{9} \mathcal{D}_{0}(z)+\frac{1}{3} \mathcal{D}_{1}(z)-\frac{\mathrm{H}(0 ; z)}{3(1-z)}+\frac{1}{z}\left(-\frac{5}{9}-\frac{1}{3} \mathrm{H}(0 ; z)-\frac{1}{3} \mathrm{H}(1 ; z)\right)\right] \\
& +\mathcal{O}(\epsilon) .
\end{aligned}
$$

\section{Rederivation of NNLO coefficient functions}

Being derived from physical matrix elements, the integrated antenna functions can be compared to results from literature for inclusive cross sections or coefficient functions, as was done previously for the final-final antennae in $[66-68,88]$. In the case of the initialfinal antennae, we can compare the integrated quark-antiquark antennae and gluon-gluon antennae against NNLO corrections to deep inelastic coefficient functions known in the literature. The former ones can be checked against DIS structure function calculations [113, $114]$ whereas the latter can be compared to the $\phi$-DIS structure functions computed in [127, 128]. The quark-gluon antennae, derived from neutralino decay, cannot be associated to any physical process and only the deepest pole could be checked against a combination of Altarelli-Parisi splitting functions.

\subsection{Deep inelastic scattering: $\gamma$ induced}

In deep inelastic scattering (DIS) the hadronic tensor $W^{\mu \nu}$ is contracted with the metric tensor $-g_{\mu \nu}$. This corresponds to the trace of the hadronic tensor, which in terms of the structure functions $\mathcal{F}_{L}$ and $\mathcal{F}_{2}$ is given by

$$
-W_{\mu}^{\mu}=-\frac{d-1}{2} \mathcal{F}_{L}\left(z, Q^{2}\right)+\frac{d-2}{2} \mathcal{F}_{2}\left(z, Q^{2}\right)
$$

where the structure functions can be expanded in powers of the strong coupling constant, following the notation of $[113,114]$ (but using $d=4-2 \epsilon$, as throughout the rest of this work):

$$
\begin{aligned}
& \mathcal{F}_{L}=\mathcal{F}_{L}^{(0)}+\frac{\alpha_{s}}{4 \pi} S_{\epsilon}\left(\frac{\mu^{2}}{Q^{2}}\right)^{\epsilon} \mathcal{F}_{L}^{(1)}+\left(\frac{\alpha_{s}}{4 \pi}\right)^{2} S_{\epsilon}^{2}\left(\frac{\mu^{2}}{Q^{2}}\right)^{2 \epsilon} \mathcal{F}_{L}^{(2)}+\mathcal{O}\left(\alpha_{s}^{3}\right), \\
& \mathcal{F}_{2}=\mathcal{F}_{2}^{(0)}+\frac{\alpha_{s}}{4 \pi} S_{\epsilon}\left(\frac{\mu^{2}}{Q^{2}}\right)^{\epsilon} \mathcal{F}_{2}^{(1)}+\left(\frac{\alpha_{s}}{4 \pi}\right)^{2} S_{\epsilon}^{2}\left(\frac{\mu^{2}}{Q^{2}}\right)^{2 \epsilon} \mathcal{F}_{2}^{(2)}+\mathcal{O}\left(\alpha_{s}^{3}\right)
\end{aligned}
$$

and $S_{\epsilon}=\exp \left[\epsilon\left(\ln (4 \pi)-\gamma_{E}\right)\right]$. For clarity, we drop the dependence on $z$ and $Q^{2}$. The factors $S_{\epsilon}$ and $\mu$ are the conventional factors appearing in dimensional regularization.

To zeroth order in $\alpha_{s}$ the structure functions are given by the simple parton model result

$$
\mathcal{F}_{L, q}^{(0)}=\mathcal{F}_{L, g}^{(0)}=0, \quad \mathcal{F}_{2, q}^{(0)}=\delta(1-z), \quad \mathcal{F}_{2, g}^{(0)}=0 .
$$

Since the overall normalization of the antenna functions is given by the leading order antenna $\mathcal{A}_{q, q}=\delta(1-z)$, we find that the correct normalization of $W_{\mu}^{\mu}$ to be checked against the antennae is

$$
-\frac{2}{d-2} W_{\mu}^{\mu}=\mathcal{F}_{2}-\frac{d-1}{d-2} \mathcal{F}_{L} .
$$


This last equation at order $\alpha_{s}$ and $\alpha_{s}^{2}$ can be compared to a linear combinations of NLO and NNLO antennae respectively. For completeness and clarity, before giving the explicit relations between the structure functions and the antennae, we report the expressions of the structure functions in terms of the Altarelli-Parisi splitting functions and the other coefficient functions $c_{i}$ and $a_{i}$. We take them from [113, 114], adjusting to $d=4-2 \epsilon$ and $\beta_{0}=\frac{11}{6} C_{A}-\frac{1}{3} N_{F}$.

$$
\begin{aligned}
& \mathcal{F}_{L, g}^{(1)}=N_{F}\left(c_{L, g}^{(1)}-\epsilon 2 a_{L, g}^{(1)}\right), \\
& \mathcal{F}_{2, g}^{(1)}=N_{F}\left(-\frac{1}{2 \epsilon} P_{g q}^{(0)}+c_{2, g}^{(1)}-\epsilon 2 a_{2, g}^{(1)}\right), \\
& \mathcal{F}_{L, q}^{(1)}=\left(c_{L, q}^{(1)}-\epsilon 2 a_{L, q}^{(1)}\right), \\
& \mathcal{F}_{2, q}^{(1)}=\left(-\frac{1}{2 \epsilon} P_{q q}^{(0)}+c_{2, q}^{(1)}-\epsilon 2 a_{2, q}^{(1)}\right), \\
& \mathcal{F}_{L, g}^{(2)}=N_{F}\left(\frac{1}{\epsilon}\left(2 \beta_{0} c_{L, g}^{(1)}-\frac{1}{2} P_{g g}^{(0)} \otimes c_{L, g}^{(1)}-\frac{1}{2} P_{q g}^{(0)} \otimes c_{L, q}^{(1)}\right)\right. \\
& \left.+c_{L, g}^{(2)}-4 \beta_{0} a_{L, g}^{(1)}+P_{g g}^{(0)} \otimes a_{L, g}^{(1)}+P_{q g}^{(0)} \otimes a_{L, q}^{(1)}\right), \\
& \mathcal{F}_{2, g}^{(2)}=N_{F}\left(\frac{1}{\epsilon^{2}}\left(\frac{1}{8} P_{q g}^{(0)} \otimes\left(P_{g g}^{(0)}+P_{q q}^{(0)}\right)-\frac{\beta_{0}}{2} P_{q q}^{(0)}\right)\right. \\
& +\frac{1}{\epsilon}\left(-\frac{1}{4} P_{q g}^{(1)}+2 \beta_{0} c_{2, g}^{(1)}-\frac{1}{2} P_{g g}^{(0)} \otimes c_{2, g}^{(1)}-\frac{1}{2} P_{q g}^{(0)} \otimes c_{2, q}^{(1)}\right) \\
& \left.+c_{2, g}^{(2)}-4 \beta_{0} a_{2, g}^{(1)}+P_{g g}^{(0)} \otimes a_{2, g}^{(1)}+P_{q g}^{(0)} \otimes a_{2, q}^{(1)}\right), \\
& \mathcal{F}_{L, q}^{(2) \mathrm{NS}}=\left(\frac{1}{\epsilon}\left(2 \beta_{0} c_{L, q}^{(1)}-\frac{1}{2} P_{q q}^{(0)} \otimes c_{L, q}^{(1)}\right)+c_{L, q}^{(2) \mathrm{NS}}-4 \beta_{0} a_{L, q}^{(1)}+P_{q q}^{(0)} \otimes a_{L, q}^{(1)}\right) \text {, } \\
& \mathcal{F}_{2, q}^{(2) \mathrm{NS}}=\left(\frac{1}{\epsilon^{2}}\left(\frac{1}{8} P_{q q}^{(0)} \otimes P_{q q}^{(0)}-\frac{\beta_{0}}{2} P_{q q}^{(0)}\right)\right. \\
& +\frac{1}{\epsilon}\left(-\frac{1}{4}\left(P_{q q}^{(1) \mathrm{NS}}+P_{q \bar{q}}^{(1) \mathrm{NS}}\right)+2 \beta_{0} c_{2, q}^{(1)}-\frac{1}{2} P_{q q}^{(0)} \otimes c_{2, q}^{(1)}\right) \\
& \left.+c_{2, q}^{(2) \mathrm{NS}}-4 \beta_{0} a_{2, q}^{(1)}+P_{q q}^{(0)} \otimes a_{2, q}^{(1)}\right), \\
& \mathcal{F}_{L, q}^{(2) \mathrm{PS}}=\left(-\frac{1}{2 \epsilon} P_{g q}^{(0)} \otimes c_{L, g}^{(1)}+c_{L, q}^{(2) \mathrm{PS}}+P_{g q}^{(0)} \otimes a_{L, g}^{(1)}\right), \\
& \mathcal{F}_{2, q}^{(2) \mathrm{PS}}=\left(\frac{1}{\epsilon^{2}}\left(\frac{1}{8} P_{q g}^{(0)} \otimes P_{g q}^{(0)}\right)-\frac{1}{\epsilon}\left(\frac{1}{4} P_{q q}^{(1) \mathrm{PS}}+\frac{1}{2} P_{g q}^{(0)} \otimes c_{2, g}^{(1)}\right)\right. \\
& \left.+c_{2, q}^{(2) \mathrm{PS}}+P_{g q}^{(0)} \otimes a_{2, g}^{(1)}\right) \text {. }
\end{aligned}
$$

The previous expressions are needed to check all the initial-final quark-antiquark antennae. They are still unrenormalized and are thus to be compared to the unrenormalized antenna 
functions. The following identities hold at NLO:

$$
\begin{aligned}
\frac{1}{C_{F}}\left(\mathcal{F}_{2, q}^{(1)}-\frac{3-2 \epsilon}{2-2 \epsilon} \mathcal{F}_{L, q}^{(1)}\right) & =4 \mathcal{A}_{q, g q}+\left.8 \delta(1-z) F_{q}^{(1)}\right|_{N}, \\
\frac{(2-2 \epsilon)}{N_{F}}\left(\mathcal{F}_{2, g}^{(1)}-\frac{3-2 \epsilon}{2-2 \epsilon} \mathcal{F}_{L, g}^{(1)}\right) & =-4 \mathcal{A}_{g, q \bar{q}},
\end{aligned}
$$

where $F_{q}^{(1)}$ is the one-loop quark form-factor given in [129]. The notation $\left.X\right|_{a}$ means that only the terms of $X$ proportional to $a$ are considered. The corresponding identities at NNLO are

$$
\begin{aligned}
\left.\frac{1}{C_{F}}\left(\mathcal{F}_{2, q}^{(2, \mathrm{NS})}-\frac{3-2 \epsilon}{2-2 \epsilon} \mathcal{F}_{L, q}^{(2, \mathrm{NS})}\right)\right|_{\frac{1}{N}}= & -\tilde{\mathcal{A}}_{q, g g q}^{0}+8 \tilde{\mathcal{A}}_{q, g q}^{1, U}-4 \mathcal{C}_{q, \bar{q} q \bar{q}}^{0}-2 \mathcal{C}_{\bar{q}, \bar{q} q \bar{q}}^{0}-2 \mathcal{C}_{\bar{q}, q \bar{q} \bar{q}}^{0} \\
& +\left.16 \delta(1-z)\left(F_{q}^{(2, U)}+C_{F} F_{q}^{(1) 2}\right)\right|_{\frac{1}{N}} \\
\left.\frac{1}{C_{F}}\left(\mathcal{F}_{2, q}^{(2, \mathrm{NS})}-\frac{3-2 \epsilon}{2-2 \epsilon} \mathcal{F}_{L, q}^{(2, \mathrm{NS})}\right)\right|_{N}= & 2 \mathcal{A}_{q, g g q}^{0}+8 \mathcal{A}_{q, g q}^{1, U}+\left.16 \delta(1-z)\left(F_{q}^{(2, U)}+C_{F} F_{q}^{(1) 2}\right)\right|_{N}, \\
\left.\frac{1}{C_{F}}\left(\mathcal{F}_{2, q}^{(2, \mathrm{NS})}-\frac{3-2 \epsilon}{2-2 \epsilon} \mathcal{F}_{L, q}^{(2, \mathrm{NS})}\right)\right|_{N_{F}}= & 2 \mathcal{B}_{q, q^{\prime} \bar{q}^{\prime} q}^{0}+\left.16 \delta(1-z)\left(F_{q}^{(2, U)}+C_{F} F_{q}^{(1) 2}\right)\right|_{N_{F}} \\
\frac{1}{C_{F} N_{F}}\left(\mathcal{F}_{2, q}^{(2, \mathrm{PS})}-\frac{3-2 \epsilon}{2-2 \epsilon} \mathcal{F}_{L, q}^{(2, \mathrm{PS})}\right)= & 2 \overline{\mathcal{B}}_{q, q^{\prime} \bar{q}^{\prime} q}^{0}, \\
\left.\frac{(2-2 \epsilon)}{N_{F}}\left(\mathcal{F}_{2, g}^{(2)}-\frac{3-2 \epsilon}{2-2 \epsilon} \mathcal{F}_{L, g}^{(2)}\right)\right|_{\frac{1}{N}}= & 2 \tilde{\mathcal{A}}_{g, g q \bar{q}}^{0}-8.17 \tilde{\mathcal{A}}_{g, q \bar{q}}^{1, U}, \\
\left.\frac{(2-2 \epsilon)}{N_{F}}\left(\mathcal{F}_{2, g}^{(2)}-\frac{3-2 \epsilon}{2-2 \epsilon} \mathcal{F}_{L, g}^{(2)}\right)\right|_{N}= & -4 \mathcal{A}_{g, g q \bar{q}}^{0}-8 \mathcal{A}_{g, q \bar{q}}^{1, U} .
\end{aligned}
$$

The structure functions $(7.5)-(7.14)$ are given in $[113,114]$ in unrenormalized form. To compare with our results, we thus considered the one-loop unrenormalized antenna functions (obtained by undoing the renormalization) the two-loop unrenormalized form factor, both characterized by the label $U$. Full agreement is found with $[113,114]$.

\subsection{Deep inelastic scattering: $\phi$-scalar induced}

The gluon-gluon $\mathcal{F}_{-}, \mathcal{G}$ - and $\mathcal{H}$-type antenna functions can be checked with the deep inelastic scalar induced structure functions derived in $[127,128]$. These structure functions are obtained in an effective theory with a scalar $\phi$ coupled to the square of the gluon field strength tensor. This effective theory is very instrumental in deriving gluonic splitting functions, and is also the effective field theory describing the coupling of the Higgs boson to gluons through a loop of asymptotically heavy coloured particles. Since $\phi$ is a scalar particle the corresponding hadronic tensor has only one structure function which can be either quark- or gluon-initiated. Their perturbative expansion reads:

$$
\begin{aligned}
& \mathcal{T}_{\phi, g}=\mathcal{T}_{\phi, g}^{(0)}+\frac{\alpha_{s}}{4 \pi} S_{\epsilon}\left(\frac{\mu^{2}}{Q^{2}}\right)^{\epsilon} \mathcal{T}_{\phi, g}^{(1)}+\left(\frac{\alpha_{s}}{4 \pi}\right)^{2} S_{\epsilon}^{2}\left(\frac{\mu^{2}}{Q^{2}}\right)^{2 \epsilon} \mathcal{T}_{\phi, g}^{(2)}+\mathcal{O}\left(\alpha_{s}^{3}\right), \\
& \mathcal{T}_{\phi, q}=\mathcal{T}_{\phi, q}^{(0)}+\frac{\alpha_{s}}{4 \pi} S_{\epsilon}\left(\frac{\mu^{2}}{Q^{2}}\right)^{\epsilon} \mathcal{T}_{\phi, q}^{(1)}+\left(\frac{\alpha_{s}}{4 \pi}\right)^{2} S_{\epsilon}^{2}\left(\frac{\mu^{2}}{Q^{2}}\right)^{2 \epsilon} \mathcal{T}_{\phi, q}^{(2)}+\mathcal{O}\left(\alpha_{s}^{3}\right) .
\end{aligned}
$$


At NLO and NNLO the structure functions have the following form:

$$
\begin{aligned}
\mathcal{T}_{\phi, g}^{(1)}=( & \left.-\frac{1}{2 \epsilon} P_{g g}^{(0)}+c_{\phi, g}^{(1)}-\epsilon 2 a_{\phi, g}^{(1)}\right) \\
\mathcal{T}_{\phi, q}^{(1)}=( & \left.-\frac{1}{2 \epsilon} P_{g q}^{(0)}+c_{\phi, q}^{(1)}-\epsilon 2 a_{\phi, q}^{(1)}\right) \\
\mathcal{T}_{\phi, g}^{(2)}=( & \frac{1}{\epsilon^{2}}\left(\frac{1}{8} P_{g g}^{(0)} \otimes P_{g g}^{(0)}+\frac{N_{F}}{8} P_{g q}^{(0)} \otimes P_{q g}^{(0)}+\frac{\beta_{0}}{2} P_{g g}^{(0)}\right) \\
& +\frac{1}{\epsilon}\left(-\frac{1}{4} P_{g g}^{(1)}-\frac{1}{2} P_{g g}^{(0)} \otimes c_{\phi, g}^{(1)}-\frac{N_{F}}{2} P_{q g}^{(0)} \otimes c_{\phi, q}^{(1)}\right) \\
& \left.+c_{\phi, g}^{(2)}+P_{g g}^{(0)} \otimes a_{\phi, g}^{(1)}+N_{F} P_{q g}^{(0)} \otimes a_{\phi, q}^{(1)}\right) \\
\mathcal{T}_{\phi, q}^{(2)}=( & \frac{1}{\epsilon^{2}}\left(\frac{1}{8} P_{q q}^{(0)} \otimes P_{g q}^{(0)}+\frac{1}{8} P_{g g}^{(0)} \otimes P_{g q}^{(0)}+\frac{\beta_{0}}{2} P_{g q}^{(0)}\right) \\
& +\frac{1}{\epsilon}\left(-\frac{1}{4} P_{g q}^{(1)}-\frac{1}{2} P_{g q}^{(0)} \otimes c_{\phi, g}^{(1)}-\frac{1}{2} P_{q q}^{(0)} \otimes c_{\phi, q}^{(1)}\right) \\
& \left.+c_{\phi, q}^{(2)}+P_{g q}^{(0)} \otimes a_{\phi, g}^{(1)}+P_{q q}^{(0)} \otimes a_{\phi, q}^{(1)}\right)
\end{aligned}
$$

The explicit expressions for the coefficients $c_{\phi, i}^{(1)}, a_{\phi, i}^{(1)}$ and $c_{\phi, i}^{(2)}$, with $i=q, g$ are given in appendix B. They were derived independently in [128], and we find full agreement. In our calculation, they follow from the following identities between structure functions and antennae:

$$
\begin{aligned}
\mathcal{T}_{\phi, g}^{(1)} & =2\left(C_{A} \mathcal{F}_{g, g g}^{0}+N_{F} \mathcal{G}_{g, q \bar{q}}^{0}\right)+4 \delta(1-z) F_{g}^{(1)} \\
\frac{1}{C_{F}(1-\epsilon)} \mathcal{T}_{\phi, q}^{(1)} & =-4 \mathcal{G}_{q, q g}^{0}, \\
\left.\mathcal{T}_{\phi, g}^{(2)}\right|_{N^{2}} & =\mathcal{F}_{g, g g g}^{0}+4 \mathcal{F}_{g, g g}^{1, R}+\left.4 \delta(1-z)\left(2 F_{g}^{(2)}+F_{g}^{(1) 2}\right)\right|_{N^{2}} \\
\left.\mathcal{T}_{\phi, g}^{(2)}\right|_{N N_{F}} & =2 \mathcal{G}_{g, q \bar{q} g}^{0}+4 \mathcal{G}_{g, q \bar{q}}^{1, R}+4 \hat{\mathcal{F}}_{g, g g}^{1, R}+\left.4 \delta(1-z)\left(2 F_{g}^{(2)}+F_{g}^{(1) 2}\right)\right|_{N N_{F}} \\
\left.\mathcal{T}_{\phi, g}^{(2)}\right|_{\frac{N_{F}}{N}} & =-\tilde{\mathcal{G}}_{g, q \bar{q} g}^{0}+4 \tilde{\mathcal{G}}_{g, q \bar{q}}^{1, R}+\left.4 \delta(1-z)\left(2 F_{g}^{(2)}+F_{g}^{(1) 2}\right)\right|_{\frac{N_{F}}{N}} \\
\left.\mathcal{T}_{\phi, g}^{(2)}\right|_{N_{F}^{2}} & =4 \hat{\mathcal{G}}_{g, q \bar{q}}^{1, R}+\left.4 \delta(1-z)\left(2 F_{g}^{(2)}+F_{g}^{(1) 2}\right)\right|_{N_{F}^{2}} \\
\left.\frac{1}{C_{F}(1-\epsilon)} \mathcal{T}_{\phi, q}^{(2)}\right|_{N} & =-2 \mathcal{G}_{q, q g g}^{0}-8 \mathcal{G}_{q, q g}^{1, R} \\
\left.\frac{1}{C_{F}(1-\epsilon)} \mathcal{T}_{\phi, q}^{(2)}\right|_{\frac{1}{N}} & =\tilde{\mathcal{G}}_{q, q g g}^{0}+8 \tilde{\mathcal{G}}_{q, q g}^{1, R}-4 \tilde{\mathcal{J}}_{q, q q \bar{q}}^{0} \\
\left.\frac{1}{C_{F}(1-\epsilon)} \mathcal{T}_{\phi, q}^{(2)}\right|_{N_{F}} & =-2 \mathcal{H}_{q, q q^{\prime} \bar{q}^{\prime}}^{0}-8 \hat{\mathcal{G}}_{q, q g}^{1, R}
\end{aligned}
$$

The antenna $\tilde{\mathcal{J}}_{q, q q \bar{q}}^{0}$ comes from the interference of four quark final states with identical quark flavour, which is not contained in table 1 since it is finite in all limits. It is given by

$$
\tilde{\mathcal{J}}_{q, q q \bar{q}}^{0}=\left(-\frac{7}{4}+\frac{7}{4 z}+\frac{9 z}{8}+\left(2-\frac{2}{z}-z\right) \zeta_{3}\right)+\mathcal{O}(\epsilon)
$$


The functions $F_{g}^{(i)}(i=1,2)$ are the gluon form factor at one- respectively two-loop given in [129].

\section{Conclusions and outlook}

In this paper, we have extended the NNLO antenna subtraction formalism [65] to include initial-final antenna configurations, where one of the hard radiator partons is in the initial state. We described the construction of initial-final antenna subtraction terms from antenna functions, including the required phase space factorization and mappings.

The analytic integration of the NNLO initial-final antenna functions requires the derivation of inclusive $2 \rightarrow 3$ phase space integrals at tree-level and of $2 \rightarrow 2$ phase space integrals at one loop. We reduced all these integrals to a small set of master integrals, which we computed using differential equations or by direct integration. With these, we provide integrals of all NNLO initial-final antenna functions, as required for the implementation into a parton-level event generator programme. A highly non-trivial check of our results is provided by the rederivation of deep inelastic coefficient functions [113, 114, 127, 128], which can be expressed as particular linear combinations of antenna functions.

The subtraction terms presented here allow the construction of a parton-level event generator program for the calculation of NNLO corrections to jet production observables in deeply inelastic electron-proton scattering. Of foremost importance in this context is the dijet production cross section in deep inelastic scattering [130-135], which has been used at HERA to measure the gluon distribution in the proton and the strong coupling constant $\alpha_{s}$. This cross section is at present only known to NLO [119-124], which is insufficient for various reasons. The error on the extraction of $\alpha_{s}$ from HERA $(2+1)$ jet data is dominated $[136,137]$ not by the statistical uncertainty on the data, but by the uncertainty inherent to the NLO calculation, as estimated by varying renormalization and factorization scales. Given that the statistical precision of the data will further improve once all data from the HERA-II run are analysed, the theoretical description requires the inclusion of the next-to-next-to-leading order (NNLO), i.e. $\mathcal{O}\left(\alpha_{s}^{3}\right)$, corrections. Moreover, present determinations of parton distribution functions [138-141] at NNLO accuracy [142, 143] can only include data sets for observables where NNLO corrections are known [113, 114, 144-147]. Consequently, the precise HERA deep inelastic dijet data could be used in global NNLO determinations only once the NNLO QCD corrections to this observable are computed. Using the subtraction formalism derived here, and the already known matrix elements [17, 18, 125, 126, 148-154], such a calculation can now be envisaged.

Moreover, the initial-final antenna functions derived here are an important ingredient to the calculation of NNLO corrections to jet observables at hadron colliders. In this case, all radiator configurations contribute: final-final (derived in [65]), initial-final (derived here) and initial-initial. The initial-initial antenna functions are at present known only to NLO, and work on their extension to NNLO is ongoing. 


\section{Acknowledgments}

We would like to thank Andreas Vogt and Sven Moch for providing us with the results of [128] on the NNLO $\phi$-DIS coefficient functions prior to publication, and for useful discussions. This research was supported in part by the Swiss National Science Foundation (SNF) under contracts PP0022-118864 and 200020-126691.

\section{A Master integrals}

The common prefactor $S_{\Gamma}$ is defined as:

$$
S_{\Gamma}=\left(\frac{(4 \pi)^{\epsilon}}{16 \pi^{2} \Gamma(1-\epsilon)}\right)^{2} .
$$

\section{A.1 Master integrals for double real radiation}

The double real master integrals expanded to the needed order in $\epsilon$ are given by:

$$
\begin{aligned}
I_{0}= & S_{\Gamma}\left(Q^{2}\right)^{1-2 \epsilon}(1-x)^{1-2 \epsilon} x^{-1} \pi \\
& {\left[1+\epsilon\left(\frac{13}{2}+2 \mathrm{H}(0 ; x)\right)+\epsilon^{2}\left(\frac{115}{4}-\frac{2}{3} \pi^{2}+13 \mathrm{H}(0 ; x)+4 \mathrm{H}(0,0 ; x)\right)\right.} \\
& +\epsilon^{3}\left(\frac{865}{8}-\frac{13}{3} \pi^{2}-10 \zeta_{3}+\frac{115}{2} \mathrm{H}(0 ; x)-\frac{4}{3} \pi^{2} \mathrm{H}(0 ; x)+26 \mathrm{H}(0,0 ; x)+8 \mathrm{H}(0,0,0 ; x)\right) \\
& +\epsilon^{4}\left(\frac{5971}{16}-\frac{115}{6} \pi^{2}-65 \zeta_{3}-\frac{\pi^{4}}{30}+\frac{865}{4} \mathrm{H}(0 ; x)-\frac{26}{3} \pi^{2} \mathrm{H}(0 ; x)-20 \mathrm{H}(0 ; x) \zeta_{3}\right. \\
& \left.\left.+115 \mathrm{H}(0,0 ; x)-\frac{8}{3} \pi^{2} \mathrm{H}(0,0 ; x)+52 \mathrm{H}(0,0,0 ; x)+16 \mathrm{H}(0,0,0,0 ; x)\right)+\mathcal{O}\left(\epsilon^{5}\right)\right] \\
I_{2}= & S_{\Gamma}\left(Q^{2}\right)^{-2 \epsilon}(1-x)^{-2 \epsilon} \pi \\
& {\left[2 \mathrm{H}(0 ; x)-\epsilon\left(\frac{2}{3} \pi^{2}-10 \mathrm{H}(0 ; x)-6 \mathrm{H}(0,0 ; x)+4 \mathrm{H}(1,0 ; x)\right)-\epsilon^{2}\left(\frac{10}{3} \pi^{2}+4 \zeta_{3}\right.\right.} \\
& -38 \mathrm{H}(0 ; x)+2 \pi^{2} \mathrm{H}(0 ; x)-\frac{4}{3} \pi^{2} \mathrm{H}(1 ; x)-30 \mathrm{H}(0,0 ; x)+20 \mathrm{H}(1,0 ; x)+4 \mathrm{H}(0,1,0 ; x) \\
& -14 \mathrm{H}(0,0,0 ; x)+12 \mathrm{H}(1,0,0 ; x)-8 \mathrm{H}(1,1,0 ; x))+\epsilon^{3}\left(-\frac{38}{3} \pi^{2}-20 \zeta_{3}+\frac{11}{45} \pi^{4}\right. \\
& +130 \mathrm{H}(0 ; x)-10 \pi^{2} \mathrm{H}(0 ; x)-24 \mathrm{H}(0 ; x) \zeta_{3}+\frac{20}{3} \pi^{2} \mathrm{H}(1 ; x)+8 \mathrm{H}(1 ; x) \zeta_{3} \\
& +\frac{4}{3} \pi^{2} \mathrm{H}(0,1 ; x)+114 \mathrm{H}(0,0 ; x)-\frac{14}{3} \pi^{2} \mathrm{H}(0,0 ; x)-76 \mathrm{H}(1,0 ; x)+4 \pi^{2} \mathrm{H}(1,0 ; x) \\
& -\frac{8}{3} \pi^{2} \mathrm{H}(1,1 ; x)-20 \mathrm{H}(0,1,0 ; x)-4 \mathrm{H}(0,0,1,0 ; x)+70 \mathrm{H}(0,0,0 ; x)-60 \mathrm{H}(1,0,0 ; x) \\
& +40 \mathrm{H}(1,1,0 ; x)+8 \mathrm{H}(1,0,1,0 ; x)-12 \mathrm{H}(0,1,0,0 ; x)+8 \mathrm{H}(0,1,1,0 ; x) \\
& +30 \mathrm{H}(0,0,0,0 ; x)-28 \mathrm{H}(1,0,0,0 ; x)+24 \mathrm{H}(1,1,0,0 ; x)-16 \mathrm{H}(1,1,1,0 ; x)) \\
& \left.+\mathcal{O}\left(\epsilon^{4}\right)\right], \\
I_{26} & S_{\Gamma}\left(Q^{2}\right)^{-1-2 \epsilon}(1-x)^{-2 \epsilon} x \pi
\end{aligned}
$$




$$
\begin{aligned}
& {\left[\frac{1}{\epsilon}\left(\frac{2 \pi^{2}}{3}+2 \mathrm{H}(0,0 ; x)+2 \mathrm{H}(1,0 ; x)\right)+4 \zeta_{3}-\frac{2}{3} \pi^{2} \mathrm{H}(0 ; x)-\frac{2}{3} \pi^{2} \mathrm{H}(1 ; x)\right.} \\
& -4 \mathrm{H}(0,1,0 ; x)+6 \mathrm{H}(0,0,0 ; x)+6 \mathrm{H}(1,0,0 ; x)-4 \mathrm{H}(1,1,0 ; x)+\epsilon\left(\frac{5}{9} \pi^{4}\right. \\
& -4 \mathrm{H}(0 ; x) \zeta_{3}-4 \mathrm{H}(1 ; x) \zeta_{3}+\frac{4}{3} \pi^{2} \mathrm{H}(0,1 ; x)-2 \pi^{2} \mathrm{H}(0,0 ; x)-2 \pi^{2} \mathrm{H}(1,0 ; x) \\
& +\frac{4}{3} \pi^{2} \mathrm{H}(1,1 ; x)-4 \mathrm{H}(0,0,1,0 ; x)-4 \mathrm{H}(1,0,1,0 ; x)-12 \mathrm{H}(0,1,0,0 ; x) \\
& +8 \mathrm{H}(0,1,1,0 ; x)+14 \mathrm{H}(0,0,0,0 ; x)+14 \mathrm{H}(1,0,0,0 ; x)-12 \mathrm{H}(1,1,0,0 ; x) \\
& \left.+8 \mathrm{H}(1,1,1,0 ; x))+\mathcal{O}\left(\epsilon^{2}\right)\right], \\
& I_{249}=S_{\Gamma}\left(Q^{2}\right)^{-2 \epsilon-2}(1-x)^{-2 \epsilon} x^{2} \pi \\
& {\left[-\frac{2}{\epsilon^{3}}-\frac{8}{\epsilon^{2}} \mathrm{H}(0 ; x)+\frac{1}{\epsilon}\left(\frac{10}{3} \pi^{2}+10 \mathrm{H}(1,0 ; x)-22 \mathrm{H}(0,0 ; x)\right)+56 \zeta_{3}+10 \pi^{2} \mathrm{H}(0 ; x)\right.} \\
& -\frac{10}{3} \pi^{2} \mathrm{H}(1 ; x)+20 \mathrm{H}(0,1,0 ; x)-50 \mathrm{H}(0,0,0 ; x)+38 \mathrm{H}(1,0,0 ; x)-20 \mathrm{H}(1,1,0 ; x) \\
& +\epsilon\left(-\frac{56}{45} \pi^{4}+156 \zeta_{3} \mathrm{H}(0 ; x)-68 \mathrm{H}(1 ; x) \zeta_{3}-\frac{20}{3} \pi^{2} \mathrm{H}(0,1 ; x)+22 \pi^{2} \mathrm{H}(0,0 ; x)\right. \\
& -18 \pi^{2} \mathrm{H}(1,0 ; x)+\frac{20}{3} \pi^{2} \mathrm{H}(1,1 ; x)+28 \mathrm{H}(0,0,1,0 ; x)-52 \mathrm{H}(1,0,1,0 ; x) \\
& +76 \mathrm{H}(0,1,0,0 ; x)-40 \mathrm{H}(0,1,1,0 ; x)-106 \mathrm{H}(0,0,0,0 ; x)+94 \mathrm{H}(1,0,0,0 ; x) \\
& \left.-76 \mathrm{H}(1,1,0,0 ; x)+40 \mathrm{H}(1,1,1,0 ; x))+\mathcal{O}\left(\epsilon^{2}\right)\right] \text {, } \\
& I_{125}=S_{\Gamma}\left(Q^{2}\right)^{-2-2 \epsilon}(1-x)^{-2 \epsilon}(1+x)^{-1} x^{2} \pi \\
& {\left[\frac{2}{\epsilon^{2}} \mathrm{H}(0 ; x)+\frac{1}{\epsilon}\left(-\pi^{2}-4 \mathrm{H}(-1,0 ; x)+8 \mathrm{H}(0,0 ; x)-4 \mathrm{H}(1,0 ; x)\right)-14 \zeta_{3}\right.} \\
& -\frac{8}{3} \pi^{2} \mathrm{H}(0 ; x)+2 \pi^{2} \mathrm{H}(1 ; x)-8 \mathrm{H}(-2,0 ; x)+2 \pi^{2} \mathrm{H}(-1 ; x)-8 \mathrm{H}(0,1,0 ; x) \\
& +8 \mathrm{H}(-1,-1,0 ; x)-16 \mathrm{H}(-1,0,0 ; x)+8 \mathrm{H}(-1,1,0 ; x)+20 \mathrm{H}(0,0,0 ; x) \\
& +8 \mathrm{H}(1,-1,0 ; x)-16 \mathrm{H}(1,0,0 ; x)+8 \mathrm{H}(1,1,0 ; x)+\epsilon\left(-\frac{\pi^{4}}{30}+4 \pi^{2} \mathrm{H}(0,-1 ; x)\right. \\
& +4 \pi^{2} \mathrm{H}(0,1 ; x)-4 \pi^{2} \mathrm{H}(-1,-1 ; x)+\frac{16}{3} \pi^{2} \mathrm{H}(-1,0 ; x)-4 \pi^{2} \mathrm{H}(-1,1 ; x) \\
& -\frac{20}{3} \pi^{2} \mathrm{H}(0,0 ; x)-4 \pi^{2} \mathrm{H}(1,-1 ; x)+\frac{16}{3} \pi^{2} \mathrm{H}(1,0 ; x)-4 \pi^{2} \mathrm{H}(1,1 ; x) \\
& +28 \zeta_{3} \mathrm{H}(-1 ; x)-28 \zeta_{3} \mathrm{H}(0 ; x)+28 \zeta_{3} \mathrm{H}(1 ; x)-32 \mathrm{H}(0,0,-1,0 ; x) \\
& -8 \mathrm{H}(0,0,1,0 ; x)+16 \mathrm{H}(0,-1,-1,0 ; x)-32 \mathrm{H}(0,-1,0,0 ; x)+16 \mathrm{H}(0,-1,1,0 ; x) \\
& +16 \mathrm{H}(-1,0,-1,0 ; x)+16 \mathrm{H}(-1,0,1,0 ; x)+16 \mathrm{H}(1,0,-1,0 ; x)+16 \mathrm{H}(1,0,1,0 ; x) \\
& +16 \mathrm{H}(0,1,-1,0 ; x)-32 \mathrm{H}(0,1,0,0 ; x)+16 \mathrm{H}(0,1,1,0 ; x)-16 \mathrm{H}(-1,-1,-1,0 ; x) \\
& +32 \mathrm{H}(-1,-1,0,0 ; x)-16 \mathrm{H}(-1,-1,1,0 ; x)-40 \mathrm{H}(-1,0,0,0 ; x) \\
& -16 \mathrm{H}(-1,1,-1,0 ; x)+32 \mathrm{H}(-1,1,0,0 ; x)-16 \mathrm{H}(-1,1,1,0 ; x)+44 \mathrm{H}(0,0,0,0 ; x) \\
& -16 \mathrm{H}(1,-1,-1,0 ; x)+32 \mathrm{H}(1,-1,0,0 ; x)-16 \mathrm{H}(1,-1,1,0 ; x)-40 \mathrm{H}(1,0,0,0 ; x) \\
& \left.-16 \mathrm{H}(1,1,-1,0 ; x)+32 \mathrm{H}(1,1,0,0 ; x)-16 \mathrm{H}(1,1,1,0 ; x))+\mathcal{O}\left(\epsilon^{2}\right)\right], \\
& I_{235}=S_{\Gamma}\left(Q^{2}\right)^{-2-2 \epsilon}(1-x)^{-1-2 \epsilon} x^{2} \pi
\end{aligned}
$$




$$
\begin{aligned}
& {\left[-\frac{\mathrm{H}(0 ; x)}{\epsilon^{2}}-\frac{7 \mathrm{H}(0,0 ; x)}{\epsilon}+8 \zeta_{3}+\frac{7}{3} \pi^{2} \mathrm{H}(0 ; x)-8 \mathrm{H}(0,-1,0 ; x)+10 \mathrm{H}(0,1,0 ; x)\right.} \\
& -19 \mathrm{H}(0,0,0 ; x)+\epsilon\left(-\frac{2 \pi^{4}}{5}+20 \zeta_{3} \mathrm{H}(0 ; x)-32 \mathrm{H}(0,0,-1,0 ; x)+4 \pi^{2} \mathrm{H}(0,-1 ; x)\right. \\
& -2 \pi^{2} \mathrm{H}(0,1 ; x)+\frac{19}{3} \pi^{2} \mathrm{H}(0,0 ; x)+10 \mathrm{H}(0,0,1,0 ; x)+16 \mathrm{H}(0,-1,-1,0 ; x) \\
& -32 \mathrm{H}(0,-1,0,0 ; x)+16 \mathrm{H}(0,-1,1,0 ; x)+16 \mathrm{H}(0,1,-1,0 ; x)+22 \mathrm{H}(0,1,0,0 ; x) \\
& \left.-20 \mathrm{H}(0,1,1,0 ; x)-43 \mathrm{H}(0,0,0,0 ; x))+\mathcal{O}\left(\epsilon^{2}\right)\right] \\
& I_{1245}=S_{\Gamma}\left(Q^{2}\right)^{-3-2 \epsilon}(1-x)^{-2 \epsilon}(1+x)^{-1} x^{3} \pi \\
& {\left[-\frac{8 \mathrm{H}(0 ; x)}{\epsilon^{2}}+\frac{1}{\epsilon}\left(\frac{10 \pi^{2}}{3}+8 \mathrm{H}(-1,0 ; x)-28 \mathrm{H}(0,0 ; x)+16 \mathrm{H}(1,0 ; x)\right)+28 \zeta_{3}\right.} \\
& -\frac{8}{3} \pi^{2} \mathrm{H}(-1 ; x)+\frac{28}{3} \pi^{2} \mathrm{H}(0 ; x)-\frac{20}{3} \pi^{2} \mathrm{H}(1 ; x)+8 \mathrm{H}(0,-1,0 ; x)+24 \mathrm{H}(0,1,0 ; x) \\
& +24 \mathrm{H}(-1,0,0 ; x)-16 \mathrm{H}(-1,1,0 ; x)-68 \mathrm{H}(0,0,0 ; x)-16 \mathrm{H}(1,-1,0 ; x) \\
& +56 \mathrm{H}(1,0,0 ; x)-32 \mathrm{H}(1,1,0 ; x)+\epsilon\left(-\frac{49 \pi^{4}}{45}+88 \mathrm{H}(0 ; x) \zeta_{3}-56 \mathrm{H}(1 ; x) \zeta_{3}\right. \\
& -\frac{4}{3} \pi^{2} \mathrm{H}(0,-1 ; x)-\frac{28}{3} \pi^{2} \mathrm{H}(0,1 ; x)+16 \mathrm{H}(0,0,-1,0 ; x)-\frac{8}{3} \pi^{2} \mathrm{H}(-1,-1 ; x) \\
& -8 \pi^{2} \mathrm{H}(-1,0 ; x)+\frac{16}{3} \pi^{2} \mathrm{H}(-1,1 ; x)+\frac{68}{3} \pi^{2} \mathrm{H}(0,0 ; x)+\frac{16}{3} \pi^{2} \mathrm{H}(1,-1 ; x) \\
& -\frac{56}{3} \pi^{2} \mathrm{H}(1,0 ; x)+\frac{40}{3} \pi^{2} \mathrm{H}(1,1 ; x)+24 \mathrm{H}(0,0,1,0 ; x)+16 \mathrm{H}(0,-1,-1,0 ; x) \\
& +16 \mathrm{H}(0,-1,0,0 ; x)-16 \mathrm{H}(0,-1,1,0 ; x)+16 \mathrm{H}(-1,0,-1,0 ; x)-16 \mathrm{H}(-1,0,1,0 ; x) \\
& -16 \mathrm{H}(1,0,-1,0 ; x)-48 \mathrm{H}(1,0,1,0 ; x)-16 \mathrm{H}(0,1,-1,0 ; x)+80 \mathrm{H}(0,1,0,0 ; x) \\
& -48 \mathrm{H}(0,1,1,0 ; x)-32 \mathrm{H}(-1,-1,-1,0 ; x)+16 \mathrm{H}(-1,-1,0,0 ; x) \\
& +56 \mathrm{H}(-1,0,0,0 ; x)-48 \mathrm{H}(-1,1,0,0 ; x)+32 \mathrm{H}(-1,1,1,0 ; x)-148 \mathrm{H}(0,0,0,0 ; x) \\
& -48 \mathrm{H}(1,-1,0,0 ; x)+32 \mathrm{H}(1,-1,1,0 ; x)+136 \mathrm{H}(1,0,0,0 ; x)+32 \mathrm{H}(1,1,-1,0 ; x) \\
& \left.-112 \mathrm{H}(1,1,0,0 ; x)+64 \mathrm{H}(1,1,1,0 ; x))+\mathcal{O}\left(\epsilon^{2}\right)\right],
\end{aligned}
$$

$$
\begin{aligned}
I_{2356}= & S_{\Gamma}\left(Q^{2}\right)^{-3-2 \epsilon}(1-x)^{-1-2 \epsilon} x^{3} \pi \\
& {\left[-\frac{2}{\epsilon^{3}}-\frac{8 \mathrm{H}(0 ; x)}{\epsilon^{2}}+\frac{1}{\epsilon}\left(2 \pi^{2}-28 \mathrm{H}(0,0 ; x)+4 \mathrm{H}(1,0 ; x)\right)+32 \zeta_{3}+\frac{28}{3} \pi^{2} \mathrm{H}(0 ; x)\right.}
\end{aligned}
$$$$
-8 \mathrm{H}(0,-1,0 ; x)+24 \mathrm{H}(0,1,0 ; x)-68 \mathrm{H}(0,0,0 ; x)+28 \mathrm{H}(1,0,0 ; x)+\epsilon\left(256 \zeta_{3}\right.
$$$$
-\frac{43 \pi^{4}}{18}+4 \pi^{2} \mathrm{H}(0,-1 ; x)-4 \pi^{2} \mathrm{H}(0,1 ; x)+\frac{68}{3} \pi^{2} \mathrm{H}(0,0 ; x)-\frac{28}{3} \pi^{2} \mathrm{H}(1,0 ; x)
$$$$
+88 \zeta_{3} \mathrm{H}(0 ; x)-32 \zeta_{3} \mathrm{H}(1 ; x)-16 \mathrm{H}(0,0,-1,0 ; x)+24 \mathrm{H}(0,0,1,0 ; x)
$$$$
+16 \mathrm{H}(0,-1,-1,0 ; x)-32 \mathrm{H}(0,-1,0,0 ; x)+16 \mathrm{H}(0,-1,1,0 ; x)+32 \mathrm{H}(1,0,-1,0 ; x)
$$$$
-40 \mathrm{H}(1,0,1,0 ; x)+16 \mathrm{H}(0,1,-1,0 ; x)+96 \mathrm{H}(0,1,0,0 ; x)-32 \mathrm{H}(0,1,1,0 ; x)
$$

$$
\begin{aligned}
& \left.-148 \mathrm{H}(0,0,0,0 ; x)+76 \mathrm{H}(1,0,0,0 ; x))+\mathcal{O}\left(\epsilon^{2}\right)\right], \\
I_{1346}= & S_{\Gamma}\left(Q^{2}\right)^{-3-2 \epsilon}(1-x)^{-1-2 \epsilon} x^{3} \pi
\end{aligned}
$$




$$
\begin{aligned}
& {\left[-\frac{10}{\epsilon^{3}}-\frac{20 \mathrm{H}(0 ; x)}{\epsilon^{2}}+\frac{1}{\epsilon}\left(\frac{20 \pi^{2}}{3}-40 \mathrm{H}(0,0 ; x)\right)+92 \zeta_{3}+\frac{40}{3} \pi^{2} \mathrm{H}(0 ; x)-80 \mathrm{H}(0,0,0 ; x)\right.} \\
& \left.+\epsilon\left(-\frac{1}{3} \pi^{4}+184 \zeta_{3} \mathrm{H}(0 ; x)+\frac{80}{3} \pi^{2} \mathrm{H}(0,0 ; x)-160 \mathrm{H}(0,0,0,0 ; x)\right)+\mathcal{O}\left(\epsilon^{2}\right)\right]
\end{aligned}
$$

\section{A.2 Master integrals for one-loop single real radiation}

The virtual master integrals are given by:

$$
\begin{aligned}
V_{14}= & 2 \pi i S_{\Gamma}\left(Q^{2}\right)^{-2 \epsilon}(1-x)^{-\epsilon} \\
& {\left[\frac{1}{\epsilon}+4+\mathrm{H}(0 ; x)+\epsilon\left(12-\frac{\pi^{2}}{6}+4 \mathrm{H}(0 ; x)+\mathrm{H}(0,0 ; x)\right)+\epsilon^{2}\left(32-\frac{2 \pi^{2}}{3}-4 \zeta_{3}\right.\right.} \\
& \left.+12 \mathrm{H}(0 ; x)-\frac{1}{6} \pi^{2} \mathrm{H}(0 ; x)+4 \mathrm{H}(0,0 ; x)+\mathrm{H}(0,0,0 ; x)\right)+\epsilon^{3}\left(80-2 \pi^{2}-16 \zeta_{3}\right. \\
& -\frac{7 \pi^{4}}{120}+32 \mathrm{H}(0 ; x)-\frac{2}{3} \pi^{2} \mathrm{H}(0 ; x)-4 \mathrm{H}(0 ; x) \zeta_{3}+12 \mathrm{H}(0,0 ; x)-\frac{1}{6} \pi^{2} \mathrm{H}(0,0 ; x) \\
& \left.+4 \mathrm{H}(0,0,0 ; x)+\mathrm{H}(0,0,0,0 ; x))+\mathcal{O}\left(\epsilon^{4}\right)\right]
\end{aligned}
$$

$$
\begin{aligned}
V_{24}= & V_{14}(1-x)^{-\epsilon} \\
& {\left[1+\epsilon \mathrm{H}(0 ; x)+\epsilon^{2}\left(\mathrm{H}(0,0 ; x)-\frac{\pi^{2}}{2}\right)+\epsilon^{3}\left(\mathrm{H}(0,0,0 ; x)-\frac{\pi^{2}}{2} \mathrm{H}(0 ; x)\right)+\mathcal{O}\left(\epsilon^{4}\right)\right], }
\end{aligned}
$$

$$
\begin{aligned}
V_{13}= & 2 \pi i S_{\Gamma}\left(Q^{2}\right)^{-2 \epsilon}(1-x)^{-\epsilon} \\
& {\left[\frac{1}{\epsilon}+5+2 \mathrm{H}(0 ; x)+\epsilon\left(19-\frac{\pi^{2}}{3}+10 \mathrm{H}(0 ; x)+4 \mathrm{H}(0,0 ; x)\right)+\epsilon^{2}\left(65-\frac{5 \pi^{2}}{3}-8 \zeta_{3}\right.\right.} \\
& \left.+38 \mathrm{H}(0 ; x)-\frac{2}{3} \pi^{2} \mathrm{H}(0 ; x)+20 \mathrm{H}(0,0 ; x)+8 \mathrm{H}(0,0,0 ; x)\right)+\epsilon^{3}\left(211-\frac{19}{3} \pi^{2}-40 \zeta_{3}\right. \\
& -\frac{7}{45} \pi^{4}+130 \mathrm{H}(0 ; x)-\frac{10}{3} \pi^{2} \mathrm{H}(0 ; x)-16 \mathrm{H}(0 ; x) \zeta_{3}+76 \mathrm{H}(0,0 ; x)-\frac{4}{3} \pi^{2} \mathrm{H}(0,0 ; x) \\
& \left.+40 \mathrm{H}(0,0,0 ; x)+16 \mathrm{H}(0,0,0,0 ; x))+\mathcal{O}\left(\epsilon^{4}\right)\right]
\end{aligned}
$$




$$
\begin{aligned}
V_{134}= & 2 \pi i S_{\Gamma}\left(Q^{2}\right)^{-1-2 \epsilon}(1-x)^{-\epsilon} x \\
& {\left[\frac{1}{\epsilon}\left(\frac{\pi^{2}}{3}+\mathrm{H}(0,0 ; x)+\mathrm{H}(1,0 ; x)\right)-\zeta_{3}-\frac{1}{6} \pi^{2} \mathrm{H}(0 ; x)+\frac{1}{6} \pi^{2} \mathrm{H}(1 ; x)+3 \mathrm{H}(0,0,0 ; x)\right.} \\
& +4 \mathrm{H}(1,0,0 ; x)+\mathrm{H}(1,1,0 ; x)+\epsilon\left(\frac{43}{360} \pi^{4}-4 \mathrm{H}(0 ; x) \zeta_{3}-5 \mathrm{H}(1 ; x) \zeta_{3}\right. \\
& -\frac{1}{2} \pi^{2} \mathrm{H}(0,0 ; x)-\frac{2}{3} \pi^{2} \mathrm{H}(1,0 ; x)+\frac{1}{6} \pi^{2} \mathrm{H}(1,1 ; x)+7 \mathrm{H}(0,0,0,0 ; x) \\
& \left.+10 \mathrm{H}(1,0,0,0 ; x)+4 \mathrm{H}(1,1,0,0 ; x)+\mathrm{H}(1,1,1,0 ; x))+\mathcal{O}\left(\epsilon^{2}\right)\right],
\end{aligned}
$$

$C_{1234}=2 \pi i S_{\Gamma}\left(Q^{2}\right)^{-2-2 \epsilon}(1-x)^{-\epsilon} x^{2}$

$$
\begin{aligned}
& {\left[-\frac{2}{\epsilon^{3}}-\frac{6}{\epsilon^{2}} \mathrm{H}(0 ; x)+\frac{1}{\epsilon}\left(\frac{5 \pi^{2}}{3}-16 \mathrm{H}(0,0 ; x)+2 \mathrm{H}(1,0 ; x)\right)+34 \zeta_{3}+\frac{16}{3} \pi^{2} \mathrm{H}(0 ; x)\right.} \\
& +\frac{1}{3} \pi^{2} \mathrm{H}(1 ; x)+8 \mathrm{H}(0,1,0 ; x)-36 \mathrm{H}(0,0,0 ; x)+12 \mathrm{H}(1,0,0 ; x)+2 \mathrm{H}(1,1,0 ; x) \\
& +\epsilon\left(-\frac{4}{9} \pi^{4}+80 \mathrm{H}(0 ; x) \zeta_{3}-22 \mathrm{H}(1 ; x) \zeta_{3}+\frac{4}{3} \pi^{2} \mathrm{H}(0,1 ; x)+\frac{34}{3} \pi^{2} \mathrm{H}(0,0 ; x)\right. \\
& -\frac{14}{3} \pi^{2} \mathrm{H}(1,0 ; x)+\frac{1}{3} \pi^{2} \mathrm{H}(1,1 ; x)+16 \mathrm{H}(0,0,1,0 ; x)-8 \mathrm{H}(1,0,1,0 ; x) \\
& +40 \mathrm{H}(0,1,0,0 ; x)+8 \mathrm{H}(0,1,1,0 ; x)-76 \mathrm{H}(0,0,0,0 ; x)+32 \mathrm{H}(1,0,0,0 ; x) \\
& \left.+4 \mathrm{H}(1,1,0,0 ; x)+2 \mathrm{H}(1,1,1,0 ; x))+\mathcal{O}\left(\epsilon^{2}\right)\right],
\end{aligned}
$$

$V_{12345}=2 \pi i S_{\Gamma}\left(Q^{2}\right)^{-3-2 \epsilon}(1-x)^{-1-2 \epsilon} x^{3}$

$$
\begin{aligned}
& {\left[-\frac{3}{\epsilon^{3}}-\frac{9}{\epsilon^{2}} \mathrm{H}(0 ; x)+\frac{1}{\epsilon}\left(\frac{13 \pi^{2}}{6}+3 \mathrm{H}(0,1 ; x)-22 \mathrm{H}(0,0 ; x)+2 \mathrm{H}(1,0 ; x)\right)+23 \zeta_{3}\right.} \\
& +\frac{17}{3} \pi^{2} \mathrm{H}(0 ; x)+\frac{1}{3} \pi^{2} \mathrm{H}(1 ; x)+10 \mathrm{H}(0,0,1 ; x)-2 \mathrm{H}(1,0,1 ; x)+10 \mathrm{H}(0,1,0 ; x) \\
& -3 \mathrm{H}(0,1,1 ; x)-48 \mathrm{H}(0,0,0 ; x)+8 \mathrm{H}(1,0,0 ; x)+\mathcal{O}(\epsilon)] .
\end{aligned}
$$

\section{B $\phi$-DIS coefficient functions}

The coefficient functions for $\phi$-DIS structure functions derived by comparing them with the antennae as done in section 7 are given by:

$$
c_{\phi, g}^{(1)}=N\left[\delta(1-z)\left(\frac{67}{9}-\frac{2 \pi^{2}}{3}\right)-\frac{11}{3} \mathcal{D}_{0}(z)+4 \mathcal{D}_{1}(z)+8 \mathrm{H}(0 ; z)+8 \mathrm{H}(1 ; z)-\frac{4 \mathrm{H}(0 ; z)}{1-z}\right.
$$




$$
\begin{aligned}
& \left.-\frac{1}{z}\left(\frac{11}{3}+4 \mathrm{H}(0 ; z)+4 \mathrm{H}(1 ; z)\right)-z(4 \mathrm{H}(0 ; z)+4 \mathrm{H}(1 ; z))+z^{2}(4 \mathrm{H}(0 ; z)+4 \mathrm{H}(1 ; z))\right] \\
& +N_{F}\left[-\frac{10}{9} \delta(1-z)+\frac{2}{3} \mathcal{D}_{0}(z)+\frac{2}{3 z}\right] \\
& a_{\phi, g}^{(1)}=N\left[-2+\pi^{2}+\delta(1-z)\left(-\frac{148}{27}+\frac{11 \pi^{2}}{24}\right)+\left(\frac{67}{18}-\frac{\pi^{2}}{2}\right) \mathcal{D}_{0}(z)-\frac{11}{6} \mathcal{D}_{1}(z)+\mathcal{D}_{2}(z)\right. \\
& -4 \mathrm{H}(0,0 ; z)-4 \mathrm{H}(0,1 ; z)-4 \mathrm{H}(1,0 ; z)-4 \mathrm{H}(1,1 ; z)+\frac{1}{1-z}\left(\frac{11}{6} \mathrm{H}(0 ; z)+2 \mathrm{H}(0,0 ; z)\right. \\
& +2 \mathrm{H}(0,1 ; z)+2 \mathrm{H}(1,0 ; z))+\frac{1}{z}\left(\frac{67}{18}-\frac{\pi^{2}}{2}+\frac{11}{6} \mathrm{H}(0 ; z)+\frac{11}{6} \mathrm{H}(1 ; z)+2 \mathrm{H}(0,0 ; z)\right. \\
& +2 \mathrm{H}(0,1 ; z)+2 \mathrm{H}(1,0 ; z)+2 \mathrm{H}(1,1 ; z))+z\left(-\frac{\pi^{2}}{2}+2 \mathrm{H}(0,0 ; z)+2 \mathrm{H}(0,1 ; z)\right. \\
& +2 \mathrm{H}(1,0 ; z)+2 \mathrm{H}(1,1 ; z))+z^{2}\left(\frac{\pi^{2}}{2}-2 \mathrm{H}(0,0 ; z)-2 \mathrm{H}(0,1 ; z)-2 \mathrm{H}(1,0 ; z)\right. \\
& -2 \mathrm{H}(1,1 ; z))] \\
& +N_{F}\left[\delta(1-z)\left(\frac{28}{27}-\frac{\pi^{2}}{12}\right)-\frac{5}{9} \mathcal{D}_{0}(z)+\frac{1}{3} \mathcal{D}_{1}(z)-\frac{\mathrm{H}(0 ; z)}{3(1-z)}-\frac{1}{z}\left(\frac{5}{9}+\frac{1}{3} \mathrm{H}(0 ; z)\right.\right. \\
& \left.\left.+\frac{1}{3} \mathrm{H}(1 ; z)\right)\right] \\
& c_{\phi, q}^{(1)}=N\left[2 \mathrm{H}(0 ; z)+2 \mathrm{H}(1 ; z)-\frac{1}{z}\left(\frac{3}{2}+2 \mathrm{H}(0 ; z)+2 \mathrm{H}(1 ; z)\right)+z(1-\mathrm{H}(0 ; z)-\mathrm{H}(1 ; z))\right] \\
& +\frac{1}{N}\left[-2 \mathrm{H}(0 ; z)-2 \mathrm{H}(1 ; z)+\frac{1}{z}\left(\frac{3}{2}+2 \mathrm{H}(0 ; z)+2 \mathrm{H}(1 ; z)\right)+z(-1+\mathrm{H}(0 ; z)\right. \\
& +\mathrm{H}(1 ; z))] \\
& a_{\phi, q}^{(1)}=N\left[-1+\frac{\pi^{2}}{4}-\mathrm{H}(0,0 ; z)-\mathrm{H}(0,1 ; z)-\mathrm{H}(1,0 ; z)-\mathrm{H}(1,1 ; z)+\frac{1}{z}\left(\frac{7}{4}-\frac{\pi^{2}}{4}+\frac{3}{4} \mathrm{H}(0 ; z)\right.\right. \\
& \left.+\frac{3}{4} \mathrm{H}(1 ; z)+\mathrm{H}(0,0 ; z)+\mathrm{H}(0,1 ; z)+\mathrm{H}(1,0 ; z)+\mathrm{H}(1,1 ; z)\right)+z\left(-\frac{\pi^{2}}{8}-\frac{1}{2} \mathrm{H}(0 ; z)\right. \\
& \left.\left.-\frac{1}{2} \mathrm{H}(1 ; z)+\frac{1}{2} \mathrm{H}(0,0 ; z)+\frac{1}{2} \mathrm{H}(0,1 ; z)+\frac{1}{2} \mathrm{H}(1,0 ; z)+\frac{1}{2} \mathrm{H}(1,1 ; z)\right)\right] \\
& +\frac{1}{N}\left[1-\frac{\pi^{2}}{4}+\mathrm{H}(0,0 ; z)+\mathrm{H}(0,1 ; z)+\mathrm{H}(1,0 ; z)+\mathrm{H}(1,1 ; z)+\frac{1}{z}\left(-\frac{7}{4}+\frac{\pi^{2}}{4}\right.\right. \\
& \left.-\frac{3}{4} \mathrm{H}(0 ; z)-\frac{3}{4} \mathrm{H}(1 ; z)-\mathrm{H}(0,0 ; z)-\mathrm{H}(0,1 ; z)-\mathrm{H}(1,0 ; z)-\mathrm{H}(1,1 ; z)\right)+z\left(\frac{\pi^{2}}{8}\right. \\
& \left.\left.+\frac{1}{2} \mathrm{H}(0 ; z)+\frac{1}{2} \mathrm{H}(1 ; z)-\frac{1}{2} \mathrm{H}(0,0 ; z)-\frac{1}{2} \mathrm{H}(0,1 ; z)-\frac{1}{2} \mathrm{H}(1,0 ; z)-\frac{1}{2} \mathrm{H}(1,1 ; z)\right)\right] \text {, } \\
& c_{\phi, g}^{(2)}=N^{2}\left[\frac{5150}{27}-\frac{266 \pi^{2}}{9}-160 \zeta_{3}+\delta(1-z)\left(\frac{30425}{162}-\frac{389 \pi^{2}}{27}+\frac{101 \pi^{4}}{180}-\frac{242 \zeta_{3}}{3}\right)\right.
\end{aligned}
$$




$$
\begin{aligned}
& +\left(-\frac{2570}{27}+\frac{88 \pi^{2}}{9}\right) \mathcal{D}_{0}(z)+\left(\frac{778}{9}-\frac{20}{3} \pi^{2}\right) \mathcal{D}_{1}(z)-\frac{88}{3} \mathcal{D}_{2}(z)+8 \mathcal{D}_{3}(z) \\
& +\frac{32}{3} \pi^{2} \mathrm{H}(-1 ; z)+\frac{449}{9} \mathrm{H}(0 ; z)-\frac{32}{3} \pi^{2} \mathrm{H}(0 ; z)+\frac{1192}{9} \mathrm{H}(1 ; z)-\frac{40}{3} \pi^{2} \mathrm{H}(1 ; z) \\
& +16 \mathrm{H}(-1,0 ; z)+\frac{532}{3} \mathrm{H}(0,1 ; z)+202 \mathrm{H}(0,0 ; z)+136 \mathrm{H}(1,0 ; z)+136 \mathrm{H}(1,1 ; z) \\
& +64 \mathrm{H}(0,0,1 ; z)-96 \mathrm{H}(0,-1,0 ; z)-32 \mathrm{H}(-1,0,1 ; z)+112 \mathrm{H}(1,0,1 ; z) \\
& +64 \mathrm{H}(0,1,0 ; z)+80 \mathrm{H}(0,1,1 ; z)+64 \mathrm{H}(-1,-1,0 ; z)-80 \mathrm{H}(-1,0,0 ; z) \\
& +80 \mathrm{H}(1,0,0 ; z)+112 \mathrm{H}(1,1,0 ; z)+96 \mathrm{H}(1,1,1 ; z)+\frac{1}{1-z}\left(68 \zeta_{3}-\frac{778}{9} \mathrm{H}(0 ; z)\right. \\
& +\frac{28}{3} \pi^{2} \mathrm{H}(0 ; z)-\frac{176}{3} \mathrm{H}(0,1 ; z)-44 \mathrm{H}(0,0 ; z)-\frac{176}{3} \mathrm{H}(1,0 ; z)-56 \mathrm{H}(0,0,1 ; z) \\
& +24 \mathrm{H}(0,-1,0 ; z)-56 \mathrm{H}(1,0,1 ; z)-48 \mathrm{H}(0,1,0 ; z)-56 \mathrm{H}(0,1,1 ; z)-28 \mathrm{H}(0,0,0 ; z) \\
& -40 \mathrm{H}(1,0,0 ; z)-56 \mathrm{H}(1,1,0 ; z))+\frac{1}{1+z}\left(28 \zeta_{3}-\frac{16}{3} \pi^{2} \mathrm{H}(-1 ; z)+\frac{4}{3} \pi^{2} \mathrm{H}(0 ; z)\right. \\
& -8 \mathrm{H}(0,0,1 ; z)+24 \mathrm{H}(0,-1,0 ; z)+16 \mathrm{H}(-1,0,1 ; z)-32 \mathrm{H}(-1,-1,0 ; z) \\
& +40 \mathrm{H}(-1,0,0 ; z)-12 \mathrm{H}(0,0,0 ; z))+\frac{1}{z}\left(-\frac{2743}{9}+\frac{176 \pi^{2}}{9}+40 \zeta_{3}+\frac{16}{3} \pi^{2} \mathrm{H}(-1 ; z)\right. \\
& -\frac{1556}{9} \mathrm{H}(0 ; z)+8 \pi^{2} \mathrm{H}(0 ; z)-\frac{340}{3} \mathrm{H}(1 ; z)+\frac{20}{3} \pi^{2} \mathrm{H}(1 ; z)-88 \mathrm{H}(0,1 ; z) \\
& +\frac{88}{3} \mathrm{H}(-1,0 ; z)-\frac{220}{3} \mathrm{H}(0,0 ; z)-\frac{352}{3} \mathrm{H}(1,0 ; z)-\frac{352}{3} \mathrm{H}(1,1 ; z)-48 \mathrm{H}(0,0,1 ; z) \\
& -16 \mathrm{H}(-1,0,1 ; z)-56 \mathrm{H}(1,0,1 ; z)-48 \mathrm{H}(0,1,0 ; z)-56 \mathrm{H}(0,1,1 ; z) \\
& +32 \mathrm{H}(-1,-1,0 ; z)-40 \mathrm{H}(-1,0,0 ; z)-16 \mathrm{H}(0,0,0 ; z)-40 \mathrm{H}(1,0,0 ; z) \\
& -56 \mathrm{H}(1,1,0 ; z)-48 \mathrm{H}(1,1,1 ; z))+z\left(-\frac{4486}{27}+\frac{136 \pi^{2}}{9}+72 \zeta_{3}+\frac{16}{3} \pi^{2} \mathrm{H}(-1 ; z)\right. \\
& -\frac{1477}{9} \mathrm{H}(0 ; z)+\frac{56}{3} \pi^{2} \mathrm{H}(0 ; z)-\frac{662}{9} \mathrm{H}(1 ; z)+\frac{20}{3} \pi^{2} \mathrm{H}(1 ; z)-\frac{224}{3} \mathrm{H}(0,1 ; z) \\
& +16 \mathrm{H}(-1,0 ; z)-66 \mathrm{H}(0,0 ; z)-92 \mathrm{H}(1,0 ; z)-92 \mathrm{H}(1,1 ; z)-112 \mathrm{H}(0,0,1 ; z) \\
& -16 \mathrm{H}(-1,0,1 ; z)-56 \mathrm{H}(1,0,1 ; z)-80 \mathrm{H}(0,1,0 ; z)-88 \mathrm{H}(0,1,1 ; z) \\
& +32 \mathrm{H}(-1,-1,0 ; z)-40 \mathrm{H}(-1,0,0 ; z)-96 \mathrm{H}(0,0,0 ; z)-40 \mathrm{H}(1,0,0 ; z) \\
& -56 \mathrm{H}(1,1,0 ; z)-48 \mathrm{H}(1,1,1 ; z))+z^{2}\left(\frac{5747}{27}-\frac{242 \pi^{2}}{9}-96 \zeta_{3}+\frac{16}{3} \pi^{2} \mathrm{H}(-1 ; z)\right. \\
& +\frac{242}{9} \mathrm{H}(0 ; z)-\frac{32}{3} \pi^{2} \mathrm{H}(0 ; z)+\frac{778}{9} \mathrm{H}(1 ; z)-\frac{20}{3} \pi^{2} \mathrm{H}(1 ; z)+\frac{484}{3} \mathrm{H}(0,1 ; z) \\
& +\frac{88}{3} \mathrm{H}(-1,0 ; z)+\frac{572}{3} \mathrm{H}(0,0 ; z)+\frac{308}{3} \mathrm{H}(1,0 ; z)+\frac{308}{3} \mathrm{H}(1,1 ; z)+64 \mathrm{H}(0,0,1 ; z) \\
& -48 \mathrm{H}(0,-1,0 ; z)-16 \mathrm{H}(-1,0,1 ; z)+56 \mathrm{H}(1,0,1 ; z)+48 \mathrm{H}(0,1,0 ; z) \\
& +56 \mathrm{H}(0,1,1 ; z)+32 \mathrm{H}(-1,-1,0 ; z)-40 \mathrm{H}(-1,0,0 ; z)+40 \mathrm{H}(0,0,0 ; z) \\
& +40 \mathrm{H}(1,0,0 ; z)+56 \mathrm{H}(1,1,0 ; z)+48 \mathrm{H}(1,1,1 ; z))] \\
& +N N_{F}\left[\frac{431}{54}+\frac{11 \pi^{2}}{9}-4 \zeta_{3}-\delta(1-z)\left(\frac{21551}{324}-\frac{28 \pi^{2}}{9}-\frac{8 \zeta_{3}}{3}\right)+\left(\frac{233}{9}-\frac{16 \pi^{2}}{9}\right) \mathcal{D}_{0}(z)\right. \\
& -\frac{56}{3} \mathcal{D}_{1}(z)+\frac{16}{3} \mathcal{D}_{2}(z)-\frac{113}{9} \mathrm{H}(0 ; z)-\frac{4}{3} \pi^{2} \mathrm{H}(0 ; z)-\frac{163}{9} \mathrm{H}(1 ; z)-\frac{22}{3} \mathrm{H}(0,1 ; z)
\end{aligned}
$$




$$
\begin{aligned}
& -5 \mathrm{H}(0,0 ; z)-14 \mathrm{H}(1,0 ; z)-14 \mathrm{H}(1,1 ; z)+8 \mathrm{H}(0,0,1 ; z)+4 \mathrm{H}(0,1,0 ; z) \\
& +4 \mathrm{H}(0,1,1 ; z)+10 \mathrm{H}(0,0,0 ; z)+\frac{1}{1-z}\left(\frac{56}{3} \mathrm{H}(0 ; z)+\frac{32}{3} \mathrm{H}(0,1 ; z)+8 \mathrm{H}(0,0 ; z)\right. \\
& \left.+\frac{32}{3} \mathrm{H}(1,0 ; z)\right)+\frac{1}{z}\left(\frac{995}{18}-\frac{20 \pi^{2}}{9}+\frac{296}{9} \mathrm{H}(0 ; z)+\frac{296}{9} \mathrm{H}(1 ; z)+\frac{40}{3} \mathrm{H}(0,1 ; z)\right. \\
& \left.+\frac{32}{3} \mathrm{H}(0,0 ; z)+\frac{40}{3} \mathrm{H}(1,0 ; z)+\frac{40}{3} \mathrm{H}(1,1 ; z)\right)+z\left(\frac{1}{27}-\frac{22 \pi^{2}}{9}-4 \zeta_{3}+\frac{193}{9} \mathrm{H}(0 ; z)\right. \\
& -\frac{4}{3} \pi^{2} \mathrm{H}(0 ; z)+\frac{125}{9} \mathrm{H}(1 ; z)+\frac{44}{3} \mathrm{H}(0,1 ; z)+17 \mathrm{H}(0,0 ; z)+6 \mathrm{H}(1,0 ; z)+6 \mathrm{H}(1,1 ; z) \\
& +8 \mathrm{H}(0,0,1 ; z)+4 \mathrm{H}(0,1,0 ; z)+4 \mathrm{H}(0,1,1 ; z)+10 \mathrm{H}(0,0,0 ; z)) \\
& +z^{2}\left(-\frac{802}{27}+\frac{16 \pi^{2}}{9}-\frac{244}{9} \mathrm{H}(0 ; z)-\frac{244}{9} \mathrm{H}(1 ; z)-\frac{32}{3} \mathrm{H}(0,1 ; z)-\frac{32}{3} \mathrm{H}(0,0 ; z)\right. \\
& \left.\left.-\frac{32}{3} \mathrm{H}(1,0 ; z)-\frac{32}{3} \mathrm{H}(1,1 ; z)\right)\right] \\
& +\frac{N_{F}}{N}\left[-\frac{521}{18}+\pi^{2}+4 \zeta_{3}+\delta(1-z)\left(\frac{63}{4}-12 \zeta_{3}\right)-\mathcal{D}_{0}(z)-\frac{43}{3} \mathrm{H}(0 ; z)+\frac{4}{3} \pi^{2} \mathrm{H}(0 ; z)\right. \\
& -\frac{31}{3} \mathrm{H}(1 ; z)-6 \mathrm{H}(0,1 ; z)-7 \mathrm{H}(0,0 ; z)-2 \mathrm{H}(1,0 ; z)-2 \mathrm{H}(1,1 ; z)-8 \mathrm{H}(0,0,1 ; z) \\
& -4 \mathrm{H}(0,1,0 ; z)-4 \mathrm{H}(0,1,1 ; z)-10 \mathrm{H}(0,0,0 ; z)+\frac{1}{z}\left(-\frac{361}{54}+\frac{4 \pi^{2}}{9}-\frac{32}{9} \mathrm{H}(0 ; z)\right. \\
& \left.-\frac{32}{9} \mathrm{H}(1 ; z)-\frac{8}{3} \mathrm{H}(0,1 ; z)-\frac{8}{3} \mathrm{H}(0,0 ; z)-\frac{8}{3} \mathrm{H}(1,0 ; z)-\frac{8}{3} \mathrm{H}(1,1 ; z)\right)+z\left(\frac{193}{9}+\frac{2 \pi^{2}}{3}\right. \\
& +4 \zeta_{3}+\frac{1}{3} \mathrm{H}(0 ; z)+\frac{4}{3} \pi^{2} \mathrm{H}(0 ; z)+\frac{19}{3} \mathrm{H}(1 ; z)-4 \mathrm{H}(0,1 ; z)-5 \mathrm{H}(0,0 ; z)+2 \mathrm{H}(1,0 ; z) \\
& +2 \mathrm{H}(1,1 ; z)-8 \mathrm{H}(0,0,1 ; z)-4 \mathrm{H}(0,1,0 ; z)-4 \mathrm{H}(0,1,1 ; z)-10 \mathrm{H}(0,0,0 ; z)) \\
& +z^{2}\left(\frac{248}{27}-\frac{4 \pi^{2}}{9}+\frac{68}{9} \mathrm{H}(0 ; z)+\frac{68}{9} \mathrm{H}(1 ; z)+\frac{8}{3} \mathrm{H}(0,1 ; z)+\frac{8}{3} \mathrm{H}(0,0 ; z)+\frac{8}{3} \mathrm{H}(1,0 ; z)\right. \\
& \left.\left.+\frac{8}{3} \mathrm{H}(1,1 ; z)\right)\right] \\
& +N_{F}^{2}\left[\delta(1-z)\left(\frac{100}{81}-\frac{4 \pi^{2}}{27}\right)-\frac{40}{27} \mathcal{D}_{0}(z)+\frac{8}{9} \mathcal{D}_{1}(z)-\frac{8}{9(1-z)} \mathrm{H}(0 ; z)\right. \\
& \left.-\frac{1}{z}\left(\frac{40}{27}+\frac{8}{9} \mathrm{H}(0 ; z)+\frac{8}{9} \mathrm{H}(1 ; z)\right)\right] \\
& c_{\phi, q}^{(2)}=N^{2}\left[\frac{2245}{27}-\frac{191 \pi^{2}}{18}-34 \zeta_{3}+2 \pi^{2} \mathrm{H}(-1 ; z)+\frac{5}{12} \mathrm{H}(0 ; z)+\frac{247}{9} \mathrm{H}(1 ; z)-\frac{10}{3} \pi^{2} \mathrm{H}(1 ; z)\right. \\
& +\frac{191}{3} \mathrm{H}(0,1 ; z)+12 \mathrm{H}(-1,0 ; z)+\frac{259}{3} \mathrm{H}(0,0 ; z)+\frac{116}{3} \mathrm{H}(1,0 ; z)+\frac{103}{3} \mathrm{H}(1,1 ; z) \\
& -16 \mathrm{H}(0,-1,0 ; z)-8 \mathrm{H}(-1,0,1 ; z)+24 \mathrm{H}(1,0,1 ; z)+6 \mathrm{H}(0,1,0 ; z)+10 \mathrm{H}(0,1,1 ; z) \\
& +8 \mathrm{H}(-1,-1,0 ; z)-16 \mathrm{H}(-1,0,0 ; z)-15 \mathrm{H}(0,0,0 ; z)+20 \mathrm{H}(1,0,0 ; z) \\
& +24 \mathrm{H}(1,1,0 ; z)+22 \mathrm{H}(1,1,1 ; z)+\frac{1}{z}\left(-\frac{28507}{216}+\frac{133 \pi^{2}}{18}+28 \zeta_{3}+2 \pi^{2} \mathrm{H}(-1 ; z)\right.
\end{aligned}
$$




$$
\begin{aligned}
& -\frac{469}{6} \mathrm{H}(0 ; z)+\frac{10}{3} \pi^{2} \mathrm{H}(0 ; z)-32 \mathrm{H}(1 ; z)+\frac{10}{3} \pi^{2} \mathrm{H}(1 ; z)-\frac{89}{3} \mathrm{H}(0,1 ; z) \\
& +\frac{44}{3} \mathrm{H}(-1,0 ; z)-\frac{106}{3} \mathrm{H}(0,0 ; z)-\frac{133}{3} \mathrm{H}(1,0 ; z)-40 \mathrm{H}(1,1 ; z)-20 \mathrm{H}(0,0,1 ; z) \\
& -8 \mathrm{H}(-1,0,1 ; z)-24 \mathrm{H}(1,0,1 ; z)-20 \mathrm{H}(0,1,0 ; z)-24 \mathrm{H}(0,1,1 ; z) \\
& +8 \mathrm{H}(-1,-1,0 ; z)-16 \mathrm{H}(-1,0,0 ; z)-8 \mathrm{H}(0,0,0 ; z)-20 \mathrm{H}(1,0,0 ; z)-24 \mathrm{H}(1,1,0 ; z) \\
& -22 \mathrm{H}(1,1,1 ; z))+z\left(-\frac{329}{108}-\frac{4 \pi^{2}}{9}+19 \zeta_{3}+\pi^{2} \mathrm{H}(-1 ; z)-24 \mathrm{H}(0 ; z)+\frac{10}{3} \pi^{2} \mathrm{H}(0 ; z)\right. \\
& -\frac{227}{36} \mathrm{H}(1 ; z)+\frac{5}{3} \pi^{2} \mathrm{H}(1 ; z)+\frac{14}{3} \mathrm{H}(0,1 ; z)+2 \mathrm{H}(-1,0 ; z)+\frac{55}{12} \mathrm{H}(0,0 ; z) \\
& -\frac{17}{6} \mathrm{H}(1,0 ; z)-\frac{5}{3} \mathrm{H}(1,1 ; z)-20 \mathrm{H}(0,0,1 ; z)-4 \mathrm{H}(-1,0,1 ; z)-12 \mathrm{H}(1,0,1 ; z) \\
& -15 \mathrm{H}(0,1,0 ; z)-17 \mathrm{H}(0,1,1 ; z)+4 \mathrm{H}(-1,-1,0 ; z)-8 \mathrm{H}(-1,0,0 ; z)-\frac{33}{2} \mathrm{H}(0,0,0 ; z) \\
& -10 \mathrm{H}(1,0,0 ; z)-12 \mathrm{H}(1,1,0 ; z)-11 \mathrm{H}(1,1,1 ; z))+z^{2}\left(\frac{304}{27}-\frac{4 \pi^{2}}{3}-\frac{176}{9} \mathrm{H}(0 ; z)\right. \\
& \left.\left.-\frac{88}{9} \mathrm{H}(1 ; z)+8 \mathrm{H}(0,1 ; z)+\frac{8}{3} \mathrm{H}(-1,0 ; z)+\frac{32}{3} \mathrm{H}(0,0 ; z)+\frac{8}{3} \mathrm{H}(1,0 ; z)+\frac{8}{3} \mathrm{H}(1,1 ; z)\right)\right] \\
& +\left[-\frac{2947}{27}+\frac{103 \pi^{2}}{9}+36 \zeta_{3}-\frac{8}{3} \pi^{2} \mathrm{H}(-1 ; z)-\frac{25}{6} \mathrm{H}(0 ; z)+\frac{4}{3} \pi^{2} \mathrm{H}(0 ; z)-\frac{373}{9} \mathrm{H}(1 ; z)\right. \\
& +\frac{10}{3} \pi^{2} \mathrm{H}(1 ; z)-\frac{206}{3} \mathrm{H}(0,1 ; z)-8 \mathrm{H}(-1,0 ; z)-\frac{250}{3} \mathrm{H}(0,0 ; z)-\frac{140}{3} \mathrm{H}(1,0 ; z) \\
& -\frac{136}{3} \mathrm{H}(1,1 ; z)-8 \mathrm{H}(0,0,1 ; z)+24 \mathrm{H}(0,-1,0 ; z)+8 \mathrm{H}(-1,0,1 ; z)-28 \mathrm{H}(1,0,1 ; z) \\
& -12 \mathrm{H}(0,1,0 ; z)-16 \mathrm{H}(0,1,1 ; z)-16 \mathrm{H}(-1,-1,0 ; z)+20 \mathrm{H}(-1,0,0 ; z) \\
& +10 \mathrm{H}(0,0,0 ; z)-20 \mathrm{H}(1,0,0 ; z)-28 \mathrm{H}(1,1,0 ; z)-24 \mathrm{H}(1,1,1 ; z)+\frac{1}{z}\left(\frac{7525}{54}-\frac{80 \pi^{2}}{9}\right. \\
& -20 \zeta_{3}-\frac{8}{3} \pi^{2} \mathrm{H}(-1 ; z)+\frac{242}{3} \mathrm{H}(0 ; z)-4 \pi^{2} \mathrm{H}(0 ; z)+48 \mathrm{H}(1 ; z)-\frac{10}{3} \pi^{2} \mathrm{H}(1 ; z) \\
& +\frac{116}{3} \mathrm{H}(0,1 ; z)-\frac{44}{3} \mathrm{H}(-1,0 ; z)+\frac{106}{3} \mathrm{H}(0,0 ; z)+\frac{160}{3} \mathrm{H}(1,0 ; z)+52 \mathrm{H}(1,1 ; z) \\
& +24 \mathrm{H}(0,0,1 ; z)+8 \mathrm{H}(-1,0,1 ; z)+28 \mathrm{H}(1,0,1 ; z)+24 \mathrm{H}(0,1,0 ; z)+28 \mathrm{H}(0,1,1 ; z) \\
& -16 \mathrm{H}(-1,-1,0 ; z)+20 \mathrm{H}(-1,0,0 ; z)+8 \mathrm{H}(0,0,0 ; z)+20 \mathrm{H}(1,0,0 ; z) \\
& +28 \mathrm{H}(1,1,0 ; z)+24 \mathrm{H}(1,1,1 ; z))+z\left(\frac{367}{54}+\frac{\pi^{2}}{9}-16 \zeta_{3}-\frac{4}{3} \pi^{2} \mathrm{H}(-1 ; z)+\frac{39}{2} \mathrm{H}(0 ; z)\right. \\
& -4 \pi^{2} \mathrm{H}(0 ; z)+\frac{50}{9} \mathrm{H}(1 ; z)-\frac{5}{3} \pi^{2} \mathrm{H}(1 ; z)+\frac{4}{3} \mathrm{H}(0,1 ; z)+2 \mathrm{H}(-1,0 ; z)-\frac{29}{6} \mathrm{H}(0,0 ; z) \\
& +\frac{19}{3} \mathrm{H}(1,0 ; z)+\frac{23}{3} \mathrm{H}(1,1 ; z)+24 \mathrm{H}(0,0,1 ; z)+4 \mathrm{H}(-1,0,1 ; z)+14 \mathrm{H}(1,0,1 ; z) \\
& +18 \mathrm{H}(0,1,0 ; z)+20 \mathrm{H}(0,1,1 ; z)-8 \mathrm{H}(-1,-1,0 ; z)+10 \mathrm{H}(-1,0,0 ; z)+19 \mathrm{H}(0,0,0 ; z) \\
& +10 \mathrm{H}(1,0,0 ; z)+14 \mathrm{H}(1,1,0 ; z)+12 \mathrm{H}(1,1,1 ; z))+z^{2}\left(-\frac{304}{27}+\frac{4 \pi^{2}}{3}+\frac{176}{9} \mathrm{H}(0 ; z)\right. \\
& \left.\left.+\frac{88}{9} \mathrm{H}(1 ; z)-8 \mathrm{H}(0,1 ; z)-\frac{8}{3} \mathrm{H}(-1,0 ; z)-\frac{32}{3} \mathrm{H}(0,0 ; z)-\frac{8}{3} \mathrm{H}(1,0 ; z)-\frac{8}{3} \mathrm{H}(1,1 ; z)\right)\right] \\
& +\frac{1}{N^{2}}\left[26-\frac{5 \pi^{2}}{6}-2 \zeta_{3}+\frac{2}{3} \pi^{2} \mathrm{H}(-1 ; z)+\frac{15}{4} \mathrm{H}(0 ; z)-\frac{4}{3} \pi^{2} \mathrm{H}(0 ; z)+14 \mathrm{H}(1 ; z)\right.
\end{aligned}
$$




$$
\begin{aligned}
& +5 \mathrm{H}(0,1 ; z)-4 \mathrm{H}(-1,0 ; z)-3 \mathrm{H}(0,0 ; z)+8 \mathrm{H}(1,0 ; z)+11 \mathrm{H}(1,1 ; z)+8 \mathrm{H}(0,0,1 ; z) \\
& -8 \mathrm{H}(0,-1,0 ; z)+4 \mathrm{H}(1,0,1 ; z)+6 \mathrm{H}(0,1,0 ; z)+6 \mathrm{H}(0,1,1 ; z)+8 \mathrm{H}(-1,-1,0 ; z) \\
& -4 \mathrm{H}(-1,0,0 ; z)+5 \mathrm{H}(0,0,0 ; z)+4 \mathrm{H}(1,1,0 ; z)+2 \mathrm{H}(1,1,1 ; z)+\frac{1}{z}\left(-\frac{59}{8}+\frac{3 \pi^{2}}{2}\right. \\
& -8 \zeta_{3}+\frac{2}{3} \pi^{2} \mathrm{H}(-1 ; z)-\frac{5}{2} \mathrm{H}(0 ; z)+\frac{2}{3} \pi^{2} \mathrm{H}(0 ; z)-16 \mathrm{H}(1 ; z)-9 \mathrm{H}(0,1 ; z) \\
& -9 \mathrm{H}(1,0 ; z)-12 \mathrm{H}(1,1 ; z)-4 \mathrm{H}(0,0,1 ; z)-4 \mathrm{H}(1,0,1 ; z)-4 \mathrm{H}(0,1,0 ; z) \\
& -4 \mathrm{H}(0,1,1 ; z)+8 \mathrm{H}(-1,-1,0 ; z)-4 \mathrm{H}(-1,0,0 ; z)-4 \mathrm{H}(1,1,0 ; z)-2 \mathrm{H}(1,1,1 ; z)) \\
& +z\left(-\frac{15}{4}+\frac{\pi^{2}}{3}-3 \zeta_{3}+\frac{1}{3} \pi^{2} \mathrm{H}(-1 ; z)+\frac{9}{2} \mathrm{H}(0 ; z)+\frac{2}{3} \pi^{2} \mathrm{H}(0 ; z)+\frac{3}{4} \mathrm{H}(1 ; z)\right. \\
& -6 \mathrm{H}(0,1 ; z)-4 \mathrm{H}(-1,0 ; z)+\frac{1}{4} \mathrm{H}(0,0 ; z)-\frac{7}{2} \mathrm{H}(1,0 ; z)-6 \mathrm{H}(1,1 ; z)-4 \mathrm{H}(0,0,1 ; z) \\
& -2 \mathrm{H}(1,0,1 ; z)-3 \mathrm{H}(0,1,0 ; z)-3 \mathrm{H}(0,1,1 ; z)+4 \mathrm{H}(-1,-1,0 ; z)-2 \mathrm{H}(-1,0,0 ; z) \\
& \left.\left.-\frac{5}{2} \mathrm{H}(0,0,0 ; z)-2 \mathrm{H}(1,1,0 ; z)-\mathrm{H}(1,1,1 ; z)\right)\right] \\
& +N N_{F}\left[-\frac{166}{27}+\frac{4 \pi^{2}}{9}-\frac{26}{3} \mathrm{H}(0 ; z)-\frac{58}{9} \mathrm{H}(1 ; z)-\frac{8}{3} \mathrm{H}(0,1 ; z)-\frac{16}{3} \mathrm{H}(0,0 ; z)\right. \\
& -\frac{8}{3} \mathrm{H}(1,0 ; z)-\frac{4}{3} \mathrm{H}(1,1 ; z)+\frac{1}{z}\left(\frac{737}{54}-\frac{4 \pi^{2}}{9}+\frac{32}{3} \mathrm{H}(0 ; z)+\frac{58}{9} \mathrm{H}(1 ; z)+\frac{8}{3} \mathrm{H}(0,1 ; z)\right. \\
& \left.+\frac{16}{3} \mathrm{H}(0,0 ; z)+\frac{8}{3} \mathrm{H}(1,0 ; z)+\frac{4}{3} \mathrm{H}(1,1 ; z)\right)+z\left(\frac{14}{27}-\frac{2 \pi^{2}}{9}+\frac{11}{3} \mathrm{H}(0 ; z)+\frac{29}{9} \mathrm{H}(1 ; z)\right. \\
& \left.\left.+\frac{4}{3} \mathrm{H}(0,1 ; z)+\frac{8}{3} \mathrm{H}(0,0 ; z)+\frac{4}{3} \mathrm{H}(1,0 ; z)+\frac{2}{3} \mathrm{H}(1,1 ; z)\right)\right] \\
& +\frac{N_{F}}{N}\left[\frac{166}{27}-\frac{4 \pi^{2}}{9}+\frac{26}{3} \mathrm{H}(0 ; z)+\frac{58}{9} \mathrm{H}(1 ; z)+\frac{8}{3} \mathrm{H}(0,1 ; z)+\frac{16}{3} \mathrm{H}(0,0 ; z)+\frac{8}{3} \mathrm{H}(1,0 ; z)\right. \\
& +\frac{4}{3} \mathrm{H}(1,1 ; z)+\frac{1}{z}\left(-\frac{737}{54}+\frac{4 \pi^{2}}{9}-\frac{32}{3} \mathrm{H}(0 ; z)-\frac{58}{9} \mathrm{H}(1 ; z)-\frac{8}{3} \mathrm{H}(0,1 ; z)-\frac{16}{3} \mathrm{H}(0,0 ; z)\right. \\
& \left.-\frac{8}{3} \mathrm{H}(1,0 ; z)-\frac{4}{3} \mathrm{H}(1,1 ; z)\right)+z\left(-\frac{14}{27}+\frac{2 \pi^{2}}{9}-\frac{11}{3} \mathrm{H}(0 ; z)-\frac{29}{9} \mathrm{H}(1 ; z)-\frac{4}{3} \mathrm{H}(0,1 ; z)\right. \\
& \left.\left.-\frac{8}{3} \mathrm{H}(0,0 ; z)-\frac{4}{3} \mathrm{H}(1,0 ; z)-\frac{2}{3} \mathrm{H}(1,1 ; z)\right)\right] \text {. }
\end{aligned}
$$

They are in full agreement with [128].

\section{References}

[1] R.K. Ellis, W.J. Stirling and B.R. Webber, QCD and collider physics, Cambridge University Press, Cambridge U.K. (1996).

[2] G. Dissertori, I.G. Knowles and M. Schmelling, Quantum chromodynamics: high energy experiments and theory, Oxford University Press, Oxford U.K. (2003).

[3] Z. Bern, L.J. Dixon and A. Ghinculov, Two-loop correction to Bhabha scattering, Phys. Rev. D 63 (2001) 053007 [hep-ph/0010075] [SPIRES]. 
[4] C. Anastasiou, E.W.N. Glover, C. Oleari and M.E. Tejeda-Yeomans, Two-loop QCD corrections to the scattering of massless distinct quarks, Nucl. Phys. B 601 (2001) 318 [hep-ph/0010212] [SPIRES].

[5] C. Anastasiou, E.W.N. Glover, C. Oleari and M.E. Tejeda-Yeomans, Two-loop QCD corrections to massless identical quark scattering, Nucl. Phys. B 601 (2001) 341 [hep-ph/0011094] [SPIRES].

[6] C. Anastasiou, E.W.N. Glover, C. Oleari and M.E. Tejeda-Yeomans, Two-loop QCD corrections to massless quark-gluon scattering, Nucl. Phys. B 605 (2001) 486 [hep-ph/0101304] [SPIRES].

[7] E.W.N. Glover, C. Oleari and M.E. Tejeda-Yeomans, Two-loop QCD corrections to gluon-gluon scattering, Nucl. Phys. B 605 (2001) 467 [hep-ph/0102201] [SPIRES].

[8] C. Anastasiou, E.W.N. Glover and M.E. Tejeda-Yeomans, Two-loop QED and QCD corrections to massless fermion-boson scattering, Nucl. Phys. B 629 (2002) 255 [hep-ph/0201274] [SPIRES].

[9] E.W.N. Glover and M.E. Tejeda-Yeomans, Two-loop QCD helicity amplitudes for massless quark-massless gauge boson scattering, JHEP 06 (2003) 033 [hep-ph/0304169] [SPIRES].

[10] E.W.N. Glover, Two-loop QCD helicity amplitudes for massless quark-quark scattering, JHEP 04 (2004) 021 [hep-ph/0401119] [SPIRES].

[11] Z. Bern, A. De Freitas and L.J. Dixon, Two-loop amplitudes for gluon fusion into two photons, JHEP 09 (2001) 037 [hep-ph/0109078] [SPIRES].

[12] Z. Bern, A. De Freitas and L.J. Dixon, Two-loop helicity amplitudes for gluon gluon scattering in QCD and supersymmetric Yang-Mills theory, JHEP 03 (2002) 018 [hep-ph/0201161] [SPIRES].

[13] Z. Bern, A. De Freitas and L.J. Dixon, Two-loop helicity amplitudes for quark gluon scattering in QCD and gluino gluon scattering in supersymmetric Yang-Mills theory, JHEP 06 (2003) 028 [hep-ph/0304168] [SPIRES].

[14] A. De Freitas and Z. Bern, Two-loop helicity amplitudes for quark-quark scattering in QCD and gluino gluino scattering in supersymmetric Yang-Mills theory, JHEP 09 (2004) 039 [hep-ph/0409007] [SPIRES].

[15] Z. Bern, A. De Freitas, L.J. Dixon, A. Ghinculov and H.L. Wong, QCD and QED corrections to light-by-light scattering, JHEP 11 (2001) 031 [hep-ph/0109079] [SPIRES].

[16] T. Binoth, E.W.N. Glover, P. Marquard and J.J. van der Bij, Two-loop corrections to light-by-light scattering in supersymmetric QED, JHEP 05 (2002) 060 [hep-ph/0202266] [SPIRES].

[17] L.W. Garland, T. Gehrmann, E.W.N. Glover, A. Koukoutsakis and E. Remiddi, The two-loop QCD matrix element for $e^{+} e^{-} \rightarrow 3$ jets, Nucl. Phys. B 627 (2002) 107 [hep-ph/0112081] [SPIRES].

[18] L.W. Garland, T. Gehrmann, E.W.N. Glover, A. Koukoutsakis and E. Remiddi, Two-loop QCD helicity amplitudes for $e^{+} e^{-} \rightarrow 3$ jets, Nucl. Phys. B 642 (2002) 227 [hep-ph/0206067] [SPIRES].

[19] J.M. Campbell and R.K. Ellis, Next-to-leading order corrections to $W+2$ jet and $Z+2$ jet production at hadron colliders, Phys. Rev. D 65 (2002) 113007 [hep-ph/0202176] [SPIRES]. 
[20] A. Signer and L.J. Dixon, Electron positron annihilation into four jets at next-to-leading order in $\alpha_{s}$, Phys. Rev. Lett. 78 (1997) 811 [hep-ph/9609460] [SPIRES].

[21] L.J. Dixon and A. Signer, Complete $O\left(\alpha_{s}^{3}\right)$ results for $e^{+} e^{-} \rightarrow(\gamma, Z) \rightarrow$ four jets, Phys. Rev. D 56 (1997) 4031 [hep-ph/9706285] [SPIRES].

[22] Z. Nagy and Z. Trócsányi, Next-to-leading order calculation of four-jet shape variables, Phys. Rev. Lett. 79 (1997) 3604 [hep-ph/9707309] [SPIRES].

[23] J.M. Campbell, M.A. Cullen and E.W.N. Glover, Four jet event shapes in electron positron annihilation, Eur. Phys. J. C 9 (1999) 245 [hep-ph/9809429] [SPIRES].

[24] S. Weinzierl and D.A. Kosower, QCD corrections to four-jet production and three-jet structure in $e^{+} e^{-}$annihilation, Phys. Rev. D 60 (1999) 054028 [hep-ph/9901277] [SPIRES].

[25] W.B. Kilgore and W.T. Giele, Next-to-leading order gluonic three jet production at hadron colliders, Phys. Rev. D 55 (1997) 7183 [hep-ph/9610433] [SPIRES].

[26] Z. Nagy and Z. Trócsányi, Multi-jet cross sections in deep inelastic scattering at next-to-leading order, Phys. Rev. Lett. 87 (2001) 082001 [hep-ph/0104315] [SPIRES].

[27] Z. Nagy, Three-jet cross sections in hadron hadron collisions at next-to-leading order, Phys. Rev. Lett. 88 (2002) 122003 [hep-ph/0110315] [SPIRES].

[28] Z. Nagy, Next-to-leading order calculation of three jet observables in hadron hadron collision, Phys. Rev. D 68 (2003) 094002 [hep-ph/0307268] [SPIRES].

[29] Z. Bern, L.J. Dixon, D.C. Dunbar and D.A. Kosower, One-loop n-point gauge theory amplitudes, unitarity and collinear limits, Nucl. Phys. B 425 (1994) 217 [hep-ph/9403226] [SPIRES].

[30] D.A. Kosower, All-order collinear behavior in gauge theories, Nucl. Phys. B 552 (1999) 319 [hep-ph/9901201] [SPIRES].

[31] D.A. Kosower and P. Uwer, One-loop splitting amplitudes in gauge theory, Nucl. Phys. B 563 (1999) 477 [hep-ph/9903515] [SPIRES].

[32] Z. Bern, V. Del Duca and C.R. Schmidt, The infrared behavior of one-loop gluon amplitudes at next-to-next-to-leading order, Phys. Lett. B 445 (1998) 168 [hep-ph/9810409] [SPIRES].

[33] Z. Bern, V. Del Duca, W.B. Kilgore and C.R. Schmidt, The infrared behavior of one-loop QCD amplitudes at next-to-next-to-leading order, Phys. Rev. D 60 (1999) 116001 [hep-ph/9903516] [SPIRES].

[34] S. Catani and M. Grazzini, The soft-gluon current at one-loop order, Nucl. Phys. B 591 (2000) 435 [hep-ph/0007142] [SPIRES].

[35] D.A. Kosower, All-orders singular emission in gauge theories, Phys. Rev. Lett. 91 (2003) 061602 [hep-ph/0301069] [SPIRES].

[36] S. Weinzierl, Subtraction terms for one-loop amplitudes with one unresolved parton, JHEP 07 (2003) 052 [hep-ph/0306248] [SPIRES].

[37] C. Anastasiou, Z. Bern, L.J. Dixon and D.A. Kosower, Planar amplitudes in maximally supersymmetric Yang-Mills theory, Phys. Rev. Lett. 91 (2003) 251602 [hep-th/0309040] [SPIRES]. 
[38] Z. Bern, L.J. Dixon and D.A. Kosower, Two-loop $g \rightarrow$ gg splitting amplitudes in QCD, JHEP 08 (2004) 012 [hep-ph/0404293] [SPIRES].

[39] S.D. Badger and E.W.N. Glover, Two-loop splitting functions in QCD, JHEP 07 (2004) 040 [hep-ph/0405236] [SPIRES].

[40] A. Gehrmann-De Ridder and E.W.N. Glover, A complete $\mathcal{O}\left(\alpha \alpha_{s}\right)$ calculation of the photon +1 jet rate in $e^{+} e^{-}$annihilation, Nucl. Phys. B 517 (1998) 269 [hep-ph/9707224] [SPIRES].

[41] J.M. Campbell and E.W.N. Glover, Double unresolved approximations to multiparton scattering amplitudes, Nucl. Phys. B 527 (1998) 264 [hep-ph/9710255] [SPIRES].

[42] S. Catani and M. Grazzini, Collinear factorization and splitting functions for next-to-next-to-leading order QCD calculations, Phys. Lett. B 446 (1999) 143 [hep-ph/9810389] [SPIRES].

[43] S. Catani and M. Grazzini, Infrared factorization of tree level QCD amplitudes at the next-to-next-to-leading order and beyond, Nucl. Phys. B 570 (2000) 287 [hep-ph/9908523] [SPIRES].

[44] F.A. Berends and W.T. Giele, Multiple soft gluon radiation in parton processes, Nucl. Phys. B 313 (1989) 595 [SPIRES].

[45] V. Del Duca, A. Frizzo and F. Maltoni, Factorization of tree $Q C D$ amplitudes in the high-energy limit and in the collinear limit, Nucl. Phys. B 568 (2000) 211 [hep-ph/9909464] [SPIRES].

[46] T.G. Birthwright, E.W.N. Glover, V.V. Khoze and P. Marquard, Multi-gluon collinear limits from MHV diagrams, JHEP 05 (2005) 013 [hep-ph/0503063] [SPIRES].

[47] Z. Kunszt and D.E. Soper, Calculation of jet cross-sections in hadron collisions at order $\alpha_{s}^{3}$, Phys. Rev. D 46 (1992) 192 [SPIRES].

[48] S. Frixione, Z. Kunszt and A. Signer, Three jet cross-sections to next-to-leading order, Nucl. Phys. B 467 (1996) 399 [hep-ph/9512328] [SPIRES].

[49] S. Catani and M.H. Seymour, A general algorithm for calculating jet cross sections in NLO QCD, Nucl. Phys. B 485 (1997) 291 [Erratum ibid. B 510 (1998) 503] [hep-ph/9605323] [SPIRES].

[50] D.A. Kosower, Antenna factorization of gauge-theory amplitudes, Phys. Rev. D 57 (1998) 5410 [hep-ph/9710213] [SPIRES].

[51] D.A. Kosower, Antenna factorization in strongly-ordered limits, Phys. Rev. D 71 (2005) 045016 [hep-ph/0311272] [SPIRES].

[52] Z. Nagy, G. Somogyi and Z. Trócsányi, Separation of soft and collinear infrared limits of QCD squared matrix elements, hep-ph/0702273 [SPIRES].

[53] G. Somogyi, Subtraction with hadronic initial states: an NNLO-compatible scheme, JHEP 05 (2009) 016 [arXiv:0903.1218] [SPIRES].

[54] D.A. Kosower, Multiple singular emission in gauge theories, Phys. Rev. D 67 (2003) 116003 [hep-ph/0212097] [SPIRES].

[55] S. Weinzierl, Subtraction terms at NNLO, JHEP 03 (2003) 062 [hep-ph/0302180] [SPIRES]. 
[56] W.B. Kilgore, Subtraction terms for hadronic production processes at next-to-next-to-leading order, Phys. Rev. D 70 (2004) 031501 [hep-ph/0403128] [SPIRES].

[57] S. Frixione and M. Grazzini, Subtraction at NNLO, JHEP 06 (2005) 010 [hep-ph/0411399] [SPIRES].

[58] G. Somogyi, Z. Trócsányi and V. Del Duca, Matching of singly- and doubly-unresolved limits of tree-level QCD squared matrix elements, JHEP 06 (2005) 024 [hep-ph/0502226] [SPIRES].

[59] G. Somogyi, Z. Trócsányi and V. Del Duca, A subtraction scheme for computing QCD jet cross sections at NNLO: regularization of doubly-real emissions, JHEP 01 (2007) 070 [hep-ph/0609042] [SPIRES].

[60] G. Somogyi and Z. Trócsányi, A subtraction scheme for computing QCD jet cross sections at NNLO: regularization of real-virtual emission, JHEP 01 (2007) 052 [hep-ph/0609043] [SPIRES].

[61] G. Somogyi and Z. Trócsányi, A subtraction scheme for computing QCD jet cross sections at NNLO: integrating the subtraction terms I, JHEP 08 (2008) 042 [arXiv:0807.0509] [SPIRES].

[62] U. Aglietti, V. Del Duca, C. Duhr, G. Somogyi and Z. Trócsányi, Analytic integration of real-virtual counterterms in NNLO jet cross sections I, JHEP 09 (2008) 107 [arXiv: 0807.0514] [SPIRES].

[63] P. Bolzoni, S.-O. Moch, G. Somogyi and Z. Trócsányi, Analytic integration of real-virtual counterterms in NNLO jet cross sections II, JHEP 08 (2009) 079 [arXiv:0905.4390] [SPIRES].

[64] S. Catani and M. Grazzini, An NNLO subtraction formalism in hadron collisions and its application to Higgs boson production at the LHC, Phys. Rev. Lett. 98 (2007) 222002 [hep-ph/0703012] [SPIRES].

[65] A. Gehrmann-De Ridder, T. Gehrmann and E.W.N. Glover, Antenna subtraction at NNLO, JHEP 09 (2005) 056 [hep-ph/0505111] [SPIRES].

[66] A. Gehrmann-De Ridder, T. Gehrmann and E.W.N. Glover, Infrared structure of $e^{+} e^{-} \rightarrow 2$ jets at NNLO, Nucl. Phys. B 691 (2004) 195 [hep-ph/0403057] [SPIRES].

[67] A. Gehrmann-De Ridder, T. Gehrmann and E.W.N. Glover, Quark-gluon antenna functions from neutralino decay, Phys. Lett. B 612 (2005) 36 [hep-ph/0501291] [SPIRES].

[68] A. Gehrmann-De Ridder, T. Gehrmann and E.W.N. Glover, Gluon-gluon antenna functions from Higgs boson decay, Phys. Lett. B 612 (2005) 49 [hep-ph/0502110] [SPIRES].

[69] A. Gehrmann-De Ridder, T. Gehrmann, E.W.N. Glover and G. Heinrich, Infrared structure of $e^{+} e^{-} \rightarrow 3$ jets at NNLO, JHEP 11 (2007) 058 [arXiv:0710.0346] [SPIRES].

[70] A. Gehrmann-De Ridder, T. Gehrmann, E.W.N. Glover and G. Heinrich, Jet rates in electron-positron annihilation at $O\left(\alpha_{s}^{3}\right)$ in QCD, Phys. Rev. Lett. 100 (2008) 172001 [arXiv: 0802.0813] [SPIRES].

[71] S. Weinzierl, NNLO corrections to 3-jet observables in electron-positron annihilation, Phys. Rev. Lett. 101 (2008) 162001 [arXiv:0807.3241] [SPIRES].

[72] S. Weinzierl, The infrared structure of $e^{+} e^{-} \rightarrow 3$ jets at NNLO reloaded, JHEP 07 (2009) 009 [arXiv:0904.1145] [SPIRES]. 
[73] A. Gehrmann-De Ridder, T. Gehrmann, E.W.N. Glover and G. Heinrich, Second-order QCD corrections to the thrust distribution, Phys. Rev. Lett. 99 (2007) 132002 [arXiv:0707.1285] [SPIRES].

[74] A. Gehrmann-De Ridder, T. Gehrmann, E.W.N. Glover and G. Heinrich, NNLO corrections to event shapes in $e^{+} e^{-}$annihilation, JHEP 12 (2007) 094 [arXiv:0711.4711] [SPIRES].

[75] A. Gehrmann-De Ridder, T. Gehrmann, E.W.N. Glover and G. Heinrich, NNLO moments of event shapes in $e^{+} e^{-}$annihilation, JHEP 05 (2009) 106 [arXiv:0903.4658] [SPIRES].

[76] S. Weinzierl, Event shapes and jet rates in electron-positron annihilation at NNLO, JHEP 06 (2009) 041 [arXiv: 0904.1077] [SPIRES].

[77] S. Weinzierl, Moments of event shapes in electron-positron annihilation at NNLO, Phys. Rev. D 80 (2009) 094018 [arXiv:0909.5056] [SPIRES].

[78] G. Dissertori et al., First determination of the strong coupling constant using NNLO predictions for hadronic event shapes in $e^{+} e^{-}$annihilations, JHEP 02 (2008) 040 [arXiv:0712.0327] [SPIRES].

[79] G. Dissertori et al., Determination of the strong coupling constant using matched $N N L O+N L L A$ predictions for hadronic event shapes in $e^{+} e^{-}$annihilations, JHEP 08 (2009) 036 [arXiv:0906.3436] [SPIRES].

[80] G. Dissertori et al., Precise determination of the strong coupling constant at NNLO in QCD from the three-jet rate in electron-positron annihilation at LEP, arXiv:0910.4283 [SPIRES].

[81] T. Becher and M.D. Schwartz, A precise determination of $\alpha_{s}$ from LEP thrust data using effective field theory, JHEP 07 (2008) 034 [arXiv: 0803.0342] [SPIRES].

[82] R.A. Davison and B.R. Webber, Non-perturbative contribution to the thrust distribution in $e^{+} e^{-}$annihilation, Eur. Phys. J. C 59 (2009) 13 [arXiv:0809.3326] [SPIRES].

[83] JADE collaboration, S. Bethke, S. Kluth, C. Pahl and J. Schieck, Determination of the strong coupling $\alpha_{S}$ from hadronic event shapes and NNLO QCD predictions using JADE data, Eur. Phys. J. C 64 (2009) 351 [arXiv:0810.1389] [SPIRES].

[84] T. Gehrmann, M. Jaquier and G. Luisoni, Hadronization effects in event shape moments, arXiv:0911.2422 [SPIRES].

[85] W.T. Giele, D.A. Kosower and P.Z. Skands, A simple shower and matching algorithm, Phys. Rev. D 78 (2008) 014026 [arXiv:0707.3652] [SPIRES].

[86] S. Catani, L. Trentadue, G. Turnock and B.R. Webber, Resummation of large logarithms in $e^{+} e^{-}$event shape distributions, Nucl. Phys. B 407 (1993) 3 [SPIRES].

[87] T. Gehrmann, G. Luisoni and H. Stenzel, Matching NLLA+NNLO for event shape distributions, Phys. Lett. B 664 (2008) 265 [arXiv:0803.0695] [SPIRES].

[88] A. Gehrmann-De Ridder and M. Ritzmann, NLO antenna subtraction with massive fermions, JHEP 07 (2009) 041 [arXiv: 0904.3297] [SPIRES].

[89] A. Daleo, T. Gehrmann and D. Maître, Antenna subtraction with hadronic initial states, JHEP 04 (2007) 016 [hep-ph/0612257] [SPIRES].

[90] T. Binoth and G. Heinrich, An automatized algorithm to compute infrared divergent multi-loop integrals, Nucl. Phys. B 585 (2000) 741 [hep-ph/0004013] [SPIRES]. 
[91] G. Heinrich, A numerical method for NNLO calculations, Nucl. Phys. (Proc. Suppl.) 116 (2003) 368 [hep-ph/0211144] [SPIRES].

[92] C. Anastasiou, K. Melnikov and F. Petriello, A new method for real radiation at NNLO, Phys. Rev. D 69 (2004) 076010 [hep-ph/0311311] [SPIRES].

[93] T. Binoth and G. Heinrich, Numerical evaluation of phase space integrals by sector decomposition, Nucl. Phys. B 693 (2004) 134 [hep-ph/0402265] [SPIRES].

[94] C. Anastasiou, K. Melnikov and F. Petriello, Higgs boson production at hadron colliders: differential cross sections through next-to-next-to-leading order, Phys. Rev. Lett. 93 (2004) 262002 [hep-ph/0409088] [SPIRES].

[95] C. Anastasiou, K. Melnikov and F. Petriello, Fully differential Higgs boson production and the di-photon signal through next-to-next-to-leading order, Nucl. Phys. B 724 (2005) 197 [hep-ph/0501130] [SPIRES].

[96] C. Anastasiou, G. Dissertori and F. Stockli, NNLO QCD predictions for the $H \rightarrow W W \rightarrow \ell \ell \nu \nu$ signal at the LHC, JHEP 09 (2007) 018 [arXiv:0707.2373] [SPIRES].

[97] K. Melnikov and F. Petriello, The $W$ boson production cross section at the LHC through $O\left(\alpha_{s}^{2}\right)$, Phys. Rev. Lett. 96 (2006) 231803 [hep-ph/0603182] [SPIRES].

[98] M. Grazzini, NNLO predictions for the Higgs boson signal in the $H \rightarrow W W \rightarrow \ell \nu \ell \nu$ and $H \rightarrow Z Z \rightarrow 4 \ell$ decay channels, JHEP 02 (2008) 043 [arXiv:0801.3232] [SPIRES].

[99] S. Catani, L. Cieri, G. Ferrera, D. de Florian and M. Grazzini, Vector boson production at hadron colliders: a fully exclusive QCD calculation at NNLO,

Phys. Rev. Lett. 103 (2009) 082001 [arXiv:0903.2120] [SPIRES].

[100] C. Anastasiou and K. Melnikov, Higgs boson production at hadron colliders in NNLO QCD, Nucl. Phys. B 646 (2002) 220 [hep-ph/0207004] [SPIRES].

[101] F.V. Tkachov, A theorem on analytical calculability of four loop renormalization group functions, Phys. Lett. B 100 (1981) 65 [SPIRES].

[102] K.G. Chetyrkin and F.V. Tkachov, Integration by parts: the algorithm to calculate $\beta$-functions in 4 loops, Nucl. Phys. B 192 (1981) 159 [SPIRES].

[103] T. Gehrmann and E. Remiddi, Differential equations for two-loop four-point functions, Nucl. Phys. B 580 (2000) 485 [hep-ph/9912329] [SPIRES].

[104] S. Laporta, High-precision calculation of multi-loop Feynman integrals by difference equations, Int. J. Mod. Phys. A 15 (2000) 5087 [hep-ph/0102033] [SPIRES].

[105] A.V. Kotikov, Differential equations method: new technique for massive Feynman diagrams calculation, Phys. Lett. B 254 (1991) 158 [SPIRES].

[106] A.V. Kotikov, Differential equations method: the calculation of vertex type Feynman diagrams, Phys. Lett. B 259 (1991) 314 [SPIRES].

[107] A.V. Kotikov, Differential equation method: the calculation of $N$ point Feynman diagrams, Phys. Lett. B 267 (1991) 123 [SPIRES].

[108] E. Remiddi, Differential equations for Feynman graph amplitudes, Nuovo Cim. A 110 (1997) 1435 [hep-th/9711188] [SPIRES].

[109] M. Caffo, H. Czyz, S. Laporta and E. Remiddi, Master equations for master amplitudes, Acta Phys. Polon. B 29 (1998) 2627 [hep-th/9807119] [SPIRES]. 
[110] M. Caffo, H. Czyz, S. Laporta and E. Remiddi, Master equations for master amplitudes, Acta Phys. Polon. B 29 (1998) 2627 [hep-th/9807119] [SPIRES].

[111] M. Caffo, H. Czyz, S. Laporta and E. Remiddi, The master differential equations for the 2-loop sunrise selfmass amplitudes, Nuovo Cim. A 111 (1998) 365 [hep-th/9805118] [SPIRES].

[112] A. Gehrmann-De Ridder, T. Gehrmann and G. Heinrich, Four-particle phase space integrals in massless QCD, Nucl. Phys. B 682 (2004) 265 [hep-ph/0311276] [SPIRES].

[113] E.B. Zijlstra and W.L. van Neerven, Order $\alpha_{s}^{2} Q C D$ corrections to the deep inelastic proton structure functions $F_{2}$ and $F_{L}$, Nucl. Phys. B 383 (1992) 525 [SPIRES].

[114] E.B. Zijlstra and W.L. van Neerven, Order $\alpha_{s}^{2}$ correction to the structure function $F_{3}\left(x, Q^{2}\right)$ in deep inelastic neutrino-hadron scattering, Phys. Lett. B 297 (1992) 377 [SPIRES].

[115] E. Remiddi and J.A.M. Vermaseren, Harmonic polylogarithms, Int. J. Mod. Phys. A 15 (2000) 725 [hep-ph/9905237] [SPIRES].

[116] T. Gehrmann and E. Remiddi, Numerical evaluation of harmonic polylogarithms, Comput. Phys. Commun. 141 (2001) 296 [hep-ph/0107173] [SPIRES].

[117] D. Maître, HPL, a Mathematica implementation of the harmonic polylogarithms, Comput. Phys. Commun. 174 (2006) 222 [hep-ph/0507152] [SPIRES].

[118] D. Maître, Extension of HPL to complex arguments, hep-ph/0703052 [SPIRES].

[119] D. Graudenz, Three jet production in deep inelastic electron-proton scattering to order $\alpha_{s}^{2}$, Phys. Lett. B 256 (1991) 518 [SPIRES].

[120] D. Graudenz, Next-to-leading order QCD corrections to jet cross-sections and jet rates in deeply inelastic electron proton scattering, Phys. Rev. D 49 (1994) 3291 [hep-ph/9307311] [SPIRES].

[121] D. Graudenz, DISASTER++ version 1.0, hep-ph/9710244 [SPIRES].

[122] E. Mirkes and D. Zeppenfeld, Dijet production at HERA in next-to-leading order, Phys. Lett. B 380 (1996) 205 [hep-ph/9511448] [SPIRES].

[123] S. Catani and M.H. Seymour, The dipole formalism for the calculation of QCD jet cross sections at next-to-leading order, Phys. Lett. B 378 (1996) 287 [hep-ph/9602277] [SPIRES].

[124] B. Pötter, JetViP 1.1: calculating one jet and two jet cross-sections with virtual photons in NLO QCD, Comput. Phys. Commun. 119 (1999) 45 [hep-ph/9806437] [SPIRES].

[125] T. Gehrmann and E. Remiddi, Analytic continuation of massless two-loop four-point functions, Nucl. Phys. B 640 (2002) 379 [hep-ph/0207020] [SPIRES].

[126] T. Gehrmann and E.W.N. Glover, Two-loop QCD helicity amplitudes for $(2+1)$-jet production in deep inelastic scattering, Phys. Lett. B 676 (2009) 146 [arXiv:0904.2665] [SPIRES].

[127] S. Moch and J.A.M. Vermaseren, Deep inelastic structure functions at two loops, Nucl. Phys. B 573 (2000) 853 [hep-ph/9912355] [SPIRES].

[128] G. Soar, A. Vogt, S. Moch and J. Vermaseren, On Higgs-exchange DIS, physical evolution kernels and fourth-order splitting functions at large $x$, arXiv:0912.0369 [SPIRES]. 
[129] T. Gehrmann, T. Huber and D. Maître, Two-loop quark and gluon form factors in dimensional regularisation, Phys. Lett. B 622 (2005) 295 [hep-ph/0507061] [SPIRES].

[130] H1 collaboration, A. Aktas et al., Measurement of inclusive jet production in deep-inelastic scattering at high $Q^{2}$ and determination of the strong coupling, Phys. Lett. B 653 (2007) 134 [arXiv: 0706.3722] [SPIRES].

[131] H1 collaboration, C. Adloff et al., Measurement of inclusive jet cross-sections in deep-inelastic e p scattering at HERA, Phys. Lett. B 542 (2002) 193 [hep-ex/0206029] [SPIRES].

[132] H1 collaboration, C. Adloff et al., Dijet production in charged and neutral current $e^{+} p$ interactions at high $Q^{2}$, Eur. Phys. J. C 19 (2001) 429 [hep-ex/0010016] [SPIRES].

[133] ZEUS collaboration, S. Chekanov et al., Inclusive-jet and dijet cross sections in deep inelastic scattering at HERA, Nucl. Phys. B 765 (2007) 1 [hep-ex/0608048] [SPIRES].

[134] ZEUS collaboration, S. Chekanov et al., Jet-radius dependence of inclusive-jet cross sections in deep inelastic scattering at HERA, Phys. Lett. B 649 (2007) 12 [hep-ex/0701039] [SPIRES].

[135] ZEUS collaboration, S. Chekanov et al., Dijet production in neutral current deep inelastic scattering at HERA, Eur. Phys. J. C 23 (2002) 13 [hep-ex/0109029] [SPIRES].

[136] H1 collaboration, M. Gouzevitch, Jet cross sections and $\alpha_{S}$ in DIS, in Proceedings of the $16^{\text {th }}$ International Workshop on "Deep Inelastic Scattering and QCD (DIS 08)", London U.K. 2008, R. Devenish, M. Wing and R. Thorne eds., pg. 171 [SPIRES].

[137] H1 and ZEUS collaborations, T. Kluge, Combined H1-ZEUS $\alpha_{S}$ fit to jets in DIS, in Proceedings of the $16^{\text {th }}$ International Workshop on "Deep Inelastic Scattering and $Q C D$ (DIS 08)", London U.K. 2008, R. Devenish, M. Wing and R. Thorne eds., pg. 172 [SPIRES].

[138] A.D. Martin, W.J. Stirling, R.S. Thorne and G. Watt, Update of parton distributions at NNLO, Phys. Lett. B 652 (2007) 292 [arXiv:0706. 0459] [SPIRES].

[139] S. Alekhin, K. Melnikov and F. Petriello, Fixed target Drell-Yan data and NNLO QCD fits of parton distribution functions, Phys. Rev. D 74 (2006) 054033 [hep-ph/0606237] [SPIRES].

[140] P. Jimenez-Delgado and E. Reya, Dynamical NNLO parton distributions, Phys. Rev. D 79 (2009) 074023 [arXiv: 0810.4274] [SPIRES].

[141] A.D. Martin, W.J. Stirling, R.S. Thorne and G. Watt, Parton distributions for the LHC, Eur. Phys. J. C 63 (2009) 189 [arXiv:0901.0002] [SPIRES].

[142] S. Moch, J.A.M. Vermaseren and A. Vogt, The three-loop splitting functions in QCD: the non-singlet case, Nucl. Phys. B 688 (2004) 101 [hep-ph/0403192] [SPIRES].

[143] A. Vogt, S. Moch and J.A.M. Vermaseren, The three-loop splitting functions in QCD: the singlet case, Nucl. Phys. B 691 (2004) 129 [hep-ph/0404111] [SPIRES].

[144] R. Hamberg, W.L. van Neerven and T. Matsuura, A complete calculation of the order $\alpha_{s}^{2}$ correction to the Drell-Yan K factor, Nucl. Phys. B 359 (1991) 343

[Erratum ibid. B 644 (2002) 403] [SPIRES].

[145] C. Anastasiou, L.J. Dixon, K. Melnikov and F. Petriello, High precision QCD at hadron colliders: electroweak gauge boson rapidity distributions at NNLO, Phys. Rev. D 69 (2004) 094008 [hep-ph/0312266] [SPIRES]. 
[146] K. Melnikov and F. Petriello, Electroweak gauge boson production at hadron colliders through $O\left(\alpha_{s}^{2}\right)$, Phys. Rev. D 74 (2006) 114017 [hep-ph/0609070] [SPIRES].

[147] S. Alekhin and S. Moch, Higher order QCD corrections to charged-lepton deep-inelastic scattering and global fits of parton distributions, Phys. Lett. B 672 (2009) 166 [arXiv:0811.1412] [SPIRES].

[148] K. Hagiwara and D. Zeppenfeld, Amplitudes for multiparton processes involving a current at $e^{+} e^{-}, e^{ \pm} p$ and hadron colliders, Nucl. Phys. B 313 (1989) 560 [SPIRES].

[149] F.A. Berends, W.T. Giele and H. Kuijf, Exact expressions for processes involving a vector boson and up to five partons, Nucl. Phys. B 321 (1989) 39 [SPIRES].

[150] N.K. Falck, D. Graudenz and G. Kramer, Cross-section for five jet production in $e^{+} e^{-}$ annihilation, Nucl. Phys. B 328 (1989) 317 [SPIRES].

[151] Z. Bern, L.J. Dixon, D.A. Kosower and S. Weinzierl, One-loop amplitudes for $e^{+} e^{-} \rightarrow \bar{q} q \bar{Q} Q$, Nucl. Phys. B 489 (1997) 3 [hep-ph/9610370] [SPIRES].

[152] Z. Bern, L.J. Dixon and D.A. Kosower, One-loop amplitudes for $e^{+} e^{-}$to four partons, Nucl. Phys. B 513 (1998) 3 [hep-ph/9708239] [SPIRES].

[153] E.W.N. Glover and D.J. Miller, The one-loop $Q C D$ corrections for $\gamma^{*} \rightarrow Q \bar{Q} q \bar{q}$, Phys. Lett. B 396 (1997) 257 [hep-ph/9609474] [SPIRES].

[154] J.M. Campbell, E.W.N. Glover and D.J. Miller, The one-loop QCD corrections for $\gamma^{*} \rightarrow q \bar{q} g g$, Phys. Lett. B 409 (1997) 503 [hep-ph/9706297] [SPIRES]. 